\title{
WestVirginiaUniversity
}

THE RESEARCH REPOSITORY @ WVU

Graduate Theses, Dissertations, and Problem Reports

2019

\section{Novel Computational Methods for Catalytic Applications}

Gihan Uthpala Panapitiya

gupanapitiya@mix.wvu.edu

Follow this and additional works at: https://researchrepository.wvu.edu/etd

Part of the Condensed Matter Physics Commons, and the Quantum Physics Commons

\section{Recommended Citation}

Panapitiya, Gihan Uthpala, "Novel Computational Methods for Catalytic Applications" (2019). Graduate Theses, Dissertations, and Problem Reports. 4095.

https://researchrepository.wvu.edu/etd/4095

This Dissertation is protected by copyright and/or related rights. It has been brought to you by the The Research Repository @ WVU with permission from the rights-holder(s). You are free to use this Dissertation in any way that is permitted by the copyright and related rights legislation that applies to your use. For other uses you must obtain permission from the rights-holder(s) directly, unless additional rights are indicated by a Creative Commons license in the record and/ or on the work itself. This Dissertation has been accepted for inclusion in WVU Graduate Theses, Dissertations, and Problem Reports collection by an authorized administrator of The Research Repository @ WVU.

For more information, please contact researchrepository@mail.wvu.edu. 


\title{
Novel Computational Methods for Catalytic Applications
}

\author{
Gihan Uthpala Panapitiya \\ Dissertation submitted to the Eberly College of Arts and Sciences \\ at West Virginia University \\ in partial fulfillment of the requirements for the degree of \\ Doctor of Philosophy in Physics \\ James P. Lewis, Ph.D., Chair \\ Alan D. Bristow, Ph.D. \\ Aldo Romero, Ph.D. \\ Edward Flagg, Ph.D. \\ Rongchao Jin, Ph.D.
}

Department of Physics and Astronomy

Morgantown, West Virginia University

2019

Keywords: Thiolate-protected, Nanoclusters, Catalytic, Machine-learning, Adsorption, Delafossites

Copyright 2019 Gihan Uthpala Panapitiya 


\section{ABSTRACT \\ Novel Computational Methods for Catalytic Applications}

\section{Gihan Uthpala Panapitiya}

Thiolate protected nanoclusters gold nanoparticles are gaining interest of many researchers due to their promising applications in a variety of fields the development of synthesizing techniques capable of producing atomically precise nanoclusters with high purity. $\mathrm{Au}_{25}(\mathrm{SR})_{18}$ is one of the widely studied nanoclsuters due its remarkable stability. In this first part of this study, we explore the structural, electronic and catalytic properties of bimetallic $\mathrm{Au}_{25-x} \mathrm{Ag}_{x}(\mathrm{SR})_{18}($ for $\mathrm{x}=6,7,8)$. Due to the combinatorial enormity of the total number of possible alloyed isomers, we choose a randomly selected subset corresponding to each alloying level. Using $\mathrm{SCH}_{3}$ as the ligand, we find that the favorable Ag dopant locations are on the surface of the $\mathrm{Au}_{13}$ icosahedron. To study the catalytic activity in $\mathrm{Au}_{25}(\mathrm{SR})_{18}$, we used $\mathrm{SCH}_{2} \mathrm{CH}_{2} \mathrm{Ph}$ and $\mathrm{S}-\mathrm{c}-\mathrm{C}_{6} \mathrm{H}_{11}$ ligands for 6-8 and 19-23 Ag alloyed systems respectively. Using the condensed-to-atom dual descriptor functions, we show that the reduction in the catalytic activity of $\mathrm{Au}_{25-x} \mathrm{Ag}_{x}(\mathrm{SR})_{18}$ is related to the reduction in the electron donating capability of the outer shell sites.

We also propose a machine-learning model, based on the random-forest method, to predict $\mathrm{CO}$ adsorption in thiolate protected nanoclusters. Two phases of feature 
selection and training, based initially on the $\mathrm{Au}_{25}$ nanocluster, are utilized in our model. One advantage to a machine-learning approach is that correlations in defined features disentangle relationships among the various structural parameters. For example, in $\mathrm{Au}_{25}$, we find that features based on the distribution of $\mathrm{Ag}$ atoms relative to the $\mathrm{CO}$ adsorption site are the most important in predicting adsorption energies. Our machine-learning model is easily extended to other Au-based nanoclusters, and we demonstrate predictions about $\mathrm{CO}$ adsorption on $\mathrm{Ag}$-alloyed $\mathrm{Au}_{36}$ and $\mathrm{Au}_{133}$ nanoclusters.

In the final section of the thesis, Delafossite materials have been studied for a long time for photovoltaic and catalytic applications due to their wide band gaps and bipolar conductivities. These systems have forbidden fundamental band gaps which are much smaller than their apparent optical gaps. Making optical transitions permissible across the fundamental gap allows photocatalytic reactions to harness solar light in the visible region. Recently, it has been shown that B site doping can break the inversion symmetry of these delafossites allowing light absorption across the fundamental gap. We study structural and electronic properties of two Fe-doped delafossites, $\mathrm{AgAl}_{1-x} \mathrm{Fe}_{x} \mathrm{O}_{2}$ and $\mathrm{AgGa}_{1-x} \mathrm{Fe}_{x} \mathrm{O}_{2}$ (x=1\%-5\%) using high-throughput calculations. Preferable Fe dopant locations in both these systems are studied using two metrics. Based on the electronic density of states and unfolded band structures, we find that Fe impurities mainly affect the valence band edges of both these systems. New Fe states at the $\Gamma$ point can result in lower energy optical transitions compared to the undoped delafossites. Using molecular orbital plots, we confirmed that Fe doping 
affects the states only at the valence band edge, resulting in valence and conduction band edges having different parities. Thus, Fe doping can permit optical transitions that are forbidden in the pure $\mathrm{AgAlO}_{2}$ and $\mathrm{AgGaO}_{2}$. 


\section{Acknowledgment}

This thesis is dedicated my parents Piyal Panapitiya and Champa Panapitiya, without whom I would not have been where I am today. They gave me courage and strength to cope with many challenges of my life. I also sincerely thank my wife Delini Samarasinghe, who was there for me to give me emotional support when things were not happening as expected.

My deep gratitude goes to my adviser Dr. James P. Lewis, who not only provided me financial support but also motivation to achieve the goals of my research projects. His feedback on my work is a major contributing factor for the success of my research projects. I would also like to thank Dr. Alan Bristow, Dr. Aldo Romero, Dr. Edward Flagg and Dr. Rongchao Jin for their time, support and guidance as members of my committee.

My thanks also goes to Kylee Underwood who helped get started in the Lewis group and Dr. Guillermo Avendanõ-Franco for reviewing some of research work and providing useful feedback. 


\section{TABLE OF CONTENTS}

Page

LIST OF TABLES ......................... . . ix

LIST OF FIGURES . . . . . . . . . . . . . . . . . . . .

CHAPTER

I. MOTIVATION AND BACKGROUND . . . . . . . . . . . . . . 1

1.1 Gold nanoclusters . . . . . . . . . . . . . . . . . 2

1.1.0.1 Thiolated gold nanoclusters . . . . . . . . . . . 4

1.1.0.2 Bimetallic thiolated gold nanoclusters . . . . . . . 5

1.2 Delafossites . . . . . . . . . . . . . . . . . . . . 7

1.2.1 Delafossites as photocatalysts . . . . . . . . . . . 14

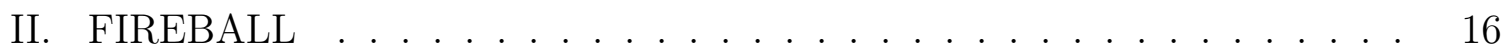

2.0.1 Approximations to exchange-correlation function . . . . . 26

2.0.2 Pseudopotentials . . . . . . . . . . . . . . . . 28

III. STRUCTURAL,ELECTRONIC AND CATALYTIC PROPERTIES

OF AG ALLOYED AU 25 NANOCLUSTER . . . . . . . . . . . . . . . 30

3.1 Introduction . . . . . . . . . . . . . . . . . . . 30

3.1.1 Computational and theoretical Tools . . . . . . . . . . . . 34

3.2 Results and discussion . . . . . . . . . . . . . . . . . 40 
$3.2 .1 \quad \mathrm{Au}_{25}(\mathrm{SR})_{18}$ nanocluster $\ldots \ldots \ldots \ldots \ldots \ldots$

$3.2 .2 \quad \mathrm{AgAu}_{24}(\mathrm{SR})_{18}$ nanocluster $\ldots \ldots \ldots \ldots \ldots$

$3.2 .3 \quad \mathrm{Ag}_{3} \mathrm{Au}_{22}(\mathrm{SR})_{18}$ nanocluster $\ldots \ldots \ldots \ldots \ldots \ldots$

3.2.4 $\operatorname{Ag}_{x} \mathrm{Au}_{25-x}(\mathrm{SR})_{18}$ nanoclusters $(\mathrm{x} \geq 3) \ldots \ldots \ldots$

3.2.5 Catalytic properties of moderately and heavily doped $\mathrm{Au}_{25} \quad 63$

3.3 Electronic and structural properties of other cluster sizes . . . . . 72

$3.3 .1 \quad \mathrm{Au}_{18}(\mathrm{SR})_{14} \ldots \ldots \ldots \ldots \ldots \ldots \ldots \ldots \ldots$

$3.3 .2 \quad \mathrm{Au}_{20}(\mathrm{SR})_{16} \ldots \ldots \ldots \ldots \ldots \ldots \ldots \ldots \ldots$

$3.3 .3 \quad \mathrm{Au}_{24}(\mathrm{SR})_{20} \ldots \ldots \ldots \ldots \ldots \ldots \ldots$

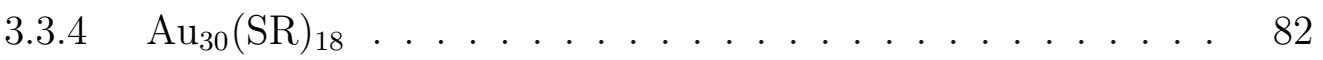

$3.3 .5 \quad \mathrm{Au}_{36} \mathrm{SR}_{24} \ldots \ldots \ldots \ldots \ldots \ldots \ldots$

$3.3 .6 \quad \mathrm{Au}_{38}(\mathrm{SR})_{24} \ldots \ldots \ldots \ldots \ldots \ldots \ldots$

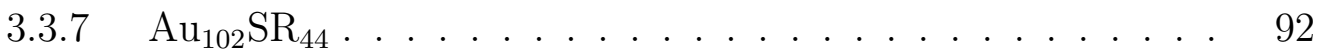

IV. PREDICTING THE ADSORPTION ENERGIES IN THIOLATE PROTECTED NANOCLUSTERS . . . . . . . . . . . . . . . 96

4.1 Introduction $\ldots \ldots \ldots \ldots \ldots \ldots \ldots$

$4.1 .1 \quad$ Machine learning . . . . . . . . . . . . . . 98

4.1.2 Machine learning based materials property prediction . . . . 99

4.2 Computational Methods . . . . . . . . . . . . . . . . . . . . . 101

4.3 Results and discussion . . . . . . . . . . . . . . . 108

4.4 Conclusions . . . . . . . . . . . . . . . . . . 127

V. STRUCTURAL AND ELECTRONIC PROPERTIES OF FE ALLOYED AG DELAFOSSITES . . . . . . . . . . . . . . . . 128 
5.1 Introduction $\ldots \ldots \ldots \ldots \ldots \ldots \ldots$

5.2 Computational Method . . . . . . . . . . . . . 130

5.3 Results and discussion . . . . . . . . . . . . . . 136

5.3.1 Structural properties of $\mathrm{AgAl}_{1-x} \mathrm{Fe}_{x} \mathrm{O}_{2}(\mathrm{x}=2 \%-5 \%) \ldots 136$

5.3.2 Structural properties of $\mathrm{AgGa}_{1-x} \mathrm{Fe}_{x} \mathrm{O}_{2}(x=2 \%-5 \%) \ldots \quad 139$

5.3 .3 Electronic properties . . . . . . . . . . . . . . . . . . 142

5.3.4 Electron transitions at the $\Gamma$ point . . . . . . . . . 154

5.4 Conclusion . . . . . . . . . . . . . . . . 157

VI. CONCLUSION . . . . . . . . . . . . . . . . . . . 159

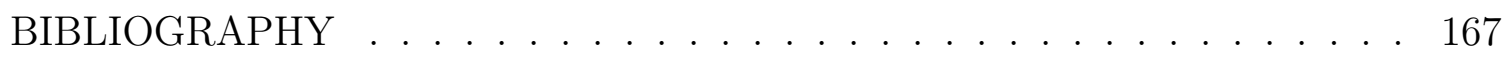

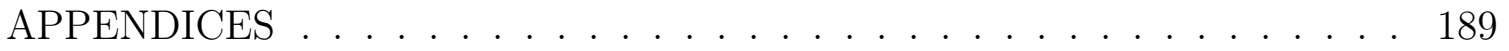

APPENDIX A. $\ldots \ldots \ldots \ldots \ldots \ldots \ldots \ldots \ldots \ldots \ldots \ldots \ldots \ldots$

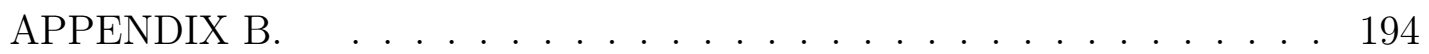

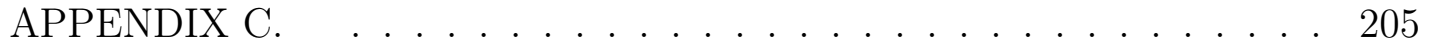




\section{LIST OF TABLES}

Table

Page

3.1 Condensed to atom Fukui function values (based on Equation 3.4) corresponding to 10 atomic sites . . . . . . . . . . . . . . 40

3.2 FIREBALL-obtained $\mathrm{f}_{k}^{-}$values corresponding to five edge and vertex sites in $\mathrm{Au}_{55}$. . . . . . . . . . . . . . . . . . . . 66

4.1 Change in the validation accuracy $\left(\mathrm{R}^{2}\right)$ with respect to the number of best features in phase 1. . . . . . . . . . . . . . . 116

4.2 Change in the validation accuracy $\left(\mathrm{R}^{2}\right)$ with respect to the number of best features in our second phase of training. . . . . . . . . . . . . 117

4.3 Effect of replacing d5 with other top features on the prediction accuracy when only two features are included in the random forest model.122

5.1 Calculated band gaps in comparison with the optical gaps . . . . . . . . 142 


\section{LIST OF FIGURES}

Figure

Page

2.1 A system with three centers. The matrix elements are zero if $\left|\mathbf{R}_{i}-\mathbf{R}_{j}\right|>$ $r_{c, i}+r_{c, j}$ corresponding to any orbital $i$ and $j \ldots . . . . . .$.

3.1 Structure of $\mathrm{Au}_{25}$ nanocluster consists of a 13-atom core surrounded by a surface layer of SR-Au-SR-Au-SR staple units. Every Au atom in the staples is bonded with a $\mathrm{Au}$ atom in the core. Color code: $\mathrm{Au}$ in the core, green and red; $\mathrm{Au}$ on the surface, blue; S, yellow; $\mathrm{C}$, gray; H, off white. . . . . . . . . . . . . 31

3.2 LUMO-HOMO electron densities of $\mathrm{Au}_{25}\left(\mathrm{SCH}_{3}\right)_{18} \ldots \ldots$. . . . . . . 40

3.3 Symmetry axis of $\mathrm{Au}_{25}(\mathrm{SR})_{18} \ldots \ldots \ldots \ldots$. . . . . . . . . . . . 42

3.4 Bond lengths in $\mathrm{Au}_{25}(\mathrm{SR})_{18} \ldots \ldots \ldots$. . . . . . . . . . . 43

3.5 Au-Au and $\mathrm{Au}-\mathrm{S}$ bonds . . . . . . . . . . . . . . . . . 43

3.6 Electronic density of states (DOS) of (a) constituent atoms and (b) core and shell $\mathrm{Au}$ atoms of $\mathrm{Au}_{25}(\mathrm{SR})_{18} \ldots \ldots$. . . . . . . . . . . 44

3.7 (a) HOMO and (b)LUMO electron densities of $\mathrm{Au}_{25}(\mathrm{SR})_{18} \ldots \ldots$

3.8 Structural energy of $1-\mathrm{Ag}$ doped $\mathrm{Au}_{25}(\mathrm{SR})_{18}$ when (a) $\mathrm{CH}_{3}$ and (b) SH were used as the ligands. Different colors indicate the location of the $\mathrm{Ag}$ atom; red:surface of the $\mathrm{Au}_{13}$, purple:shell, blue:center . . .

3.9 The effect of the location of $\mathrm{Ag}$ atoms on the total energy of $\mathrm{Au}_{22} \mathrm{Ag}_{3}\left(\mathrm{SCH}_{3}\right)_{18} \ldots \ldots \ldots . \ldots . \ldots . \ldots 48$

3.10 MALDI-MS of $\mathrm{Au}_{25-x} \mathrm{Ag}_{x}(\mathrm{SR})_{18}$ nanoalloys . . . . . . . . . . . . . 50

3.11 Lowest energy isomers of (a)6-, (b)7- and (c)8-Ag alloyed $\mathrm{Au}_{25}(\mathrm{SR})_{18} \quad$. 51 
3.12 Location of Ag atoms in (a) 6-, (b) 7- and (c) 8-Ag alloyed nanoclusters obtained with random $\mathrm{Ag}$ substitution (only 50 lowest energy structures are shown for clarity). Red color bars correspond to the number of $\mathrm{Ag}$ atoms in the 13 atoms Metal surface $\left(\mathrm{M}_{13}\right)$. Blue color bars correspond to the number of $\mathrm{Ag}$ atoms in the outer shell and green indicates a $\mathrm{Ag}$ occupancy in the center. . . . . . . . . 53

3.13 Location of the $\mathrm{Ag}$ atoms of the 10 lowest energy isomers corresponding to calculations done with $\mathrm{Ag}$ confined to the $\mathrm{Au}_{25}$ core and with randomly chosen $\mathrm{Ag}$ sites. . . . . . . . . . . . 55

3.14 Clustering factors in $2,6,7,8 \mathrm{Ag}$ alloyed $\mathrm{Au}_{25}(\mathrm{SR})_{18} \ldots \ldots \ldots$

3.15 Density of states (DOS) and partial density of states (PDOS) for (a) 0-, (b) 6-, (c) 7- and (e) 8-Ag alloyed nanoclusters in comparison with the (e) weighted average DOS and PDOS over 6,7, and 8-Ag cases. (Weights are based on the height of the peaks in the MALDI-MS (shown in Figure 3.10).) . . . . . . . . . . . . . . . . . 59

3.16 Orbital DOS of 6-,7-, and 8-Ag alloyed $\mathrm{Au}_{25}\left(\mathrm{SCH}_{3}\right)_{18} \ldots \ldots$. . . . 60

3.17 HOMO and LUMO electron densities of lowest energy isomers of $\mathrm{Au}_{25-x} \mathrm{Ag}_{x}\left(\mathrm{SCH}_{3}\right)_{18}$ for $\mathrm{x}=6,7,8$. Image orientation was decided such that the location of the Ag atoms and the superatom orbitals are visible. Brown: gold; purple: silver; yellow: sulfur. Isovalue $=0.00662$

3.18 Temperature dependent CO conversion rates . . . . . . . . . . . . . 64

3.19 Weighted average dual descriptor values corresponding unalloyed, 3-6 and 5-9 Ag alloyed $\mathrm{Au}_{25}\left(\mathrm{SCH}_{2} \mathrm{CH}_{2} \mathrm{Ph}\right)_{18} \ldots \ldots . . . . . . .668$

3.20 Current belief is that $\mathrm{O}_{2}$ species at the interracial region between $\mathrm{Au}_{25}$ and $\mathrm{CeO}_{2}$ are vital for $\mathrm{CO}$ oxidation. . . . . . . . . . . . . . 69

3.21 The (a) structure of $\mathrm{Au}_{25}\left(\mathrm{~S}-\mathrm{c}-\mathrm{C}_{6} \mathrm{H}_{11}\right)_{18}$ and the (b) CO conversion rates in the presence of $\mathrm{Ag}$ alloyed $\mathrm{Au}_{25}\left(\mathrm{~S}-\mathrm{c}-\mathrm{C}_{6} \mathrm{H}_{11}\right)_{18}$ as the catalyst. . 70

3.22 Weighted average dual descriptor values corresponding to 19-23 Ag alloyed $\mathrm{Au}_{25}\left(\mathrm{~S}-\mathrm{c}-\mathrm{C}_{6} \mathrm{H}_{11}\right)_{18}$ in comparison with unalloyed and 5-9 Ag alloyed $\mathrm{Ag}_{x} \mathrm{Au}_{25-x}\left(\mathrm{SC}_{2} \mathrm{H}_{4} \mathrm{Ph}\right)_{18}$. . . . . . . . . . . . . . . 72

3.23 Structure of $\mathrm{Au}_{18} \mathrm{SR}_{14} \ldots \ldots \ldots \ldots \ldots \ldots$. . . . . . . . . . . . . . . . . . .

3.24 Structural trends in $\mathrm{Ag}$ alloyed $\mathrm{Au}_{18} \mathrm{SR}_{14} \ldots \ldots \ldots$. . . . . . . . 75 
3.25 Density of states of $\mathrm{Ag}$ alloyed $\mathrm{Au}_{18} \mathrm{SR}_{14} \ldots \ldots \ldots$. . . . . . . . 76

3.26 Structure of $\mathrm{Au}_{20} \mathrm{SR}_{16} \ldots \ldots \ldots \ldots$. . . . . . . . . . . 77

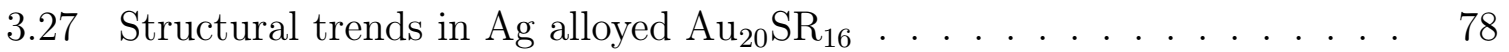

3.28 Density of states of $\mathrm{Ag}$ alloyed $\mathrm{Au}_{20} \mathrm{SR}_{16} \ldots \ldots \ldots$. . . . . . . 79

3.29 Structure of $\mathrm{Au}_{24} \mathrm{SR}_{20} \ldots \ldots \ldots \ldots$. . . . . . . . . . . 80

3.30 Structural trends in $\mathrm{Ag}$ alloyed $\mathrm{Au}_{24} \mathrm{SR}_{20} \ldots \ldots \ldots$. . . . . . . . 81

3.31 Density of states of $\mathrm{Ag}$ alloyed $\mathrm{Au}_{24} \mathrm{SR}_{20} \ldots \ldots \ldots$. . . . . . . 82

3.32 Structure of $\mathrm{Au}_{30} \mathrm{SR}_{18} \ldots \ldots \ldots \ldots$. . . . . . . . . . . . 83

3.33 Structural trends in $\mathrm{Ag}$ alloyed $\mathrm{Au}_{30} \mathrm{SR}_{18} \ldots \ldots$. . . . . . . . . . 84

3.34 Density of states of $\mathrm{Ag}$ alloyed $\mathrm{Au}_{30} \mathrm{SR}_{18} \ldots \ldots \ldots$. . . . . . . 85

3.35 Structure of $\mathrm{Au}_{36} \mathrm{SR}_{24} \ldots \ldots \ldots \ldots$. . . . . . . . . . . 86

3.36 Density of states of $\mathrm{Ag}$ alloyed $\mathrm{Au}_{36}(\mathrm{SR})_{24} \ldots \ldots \ldots$. . . . . . . 87

3.37 Density of states of $\mathrm{Ag}$ alloyed $\mathrm{Au}_{36}(\mathrm{SR})_{24} \ldots \ldots \ldots$

3.38 Structure of $\mathrm{Au}_{38} \mathrm{SR}_{24} \ldots \ldots \ldots$. . . . . . . . . . . . . . 89

3.39 Structural trends in $\mathrm{Ag}$ alloyed $\mathrm{Au}_{38} \mathrm{SR}_{24} \ldots \ldots$. . . . . . . . . . 91

3.40 Density of states of $\mathrm{Ag}$ alloyed $\mathrm{Au}_{38} \mathrm{SR}_{24}$. . . . . . . . . . . . . . 92

3.41 Structure of $\mathrm{Au}_{102} \mathrm{SR}_{44} \ldots \ldots \ldots$. . . . . . . . . . . . . . 92

3.42 Structural trends in $\mathrm{Ag}$ alloyed $\mathrm{Au}_{102} \mathrm{SR}_{44} \ldots \ldots$. . . . . . . . . . 94

3.43 Density of states of $\mathrm{Ag}$ alloyed $\mathrm{Au}_{102} \mathrm{SR}_{44} \ldots \ldots \ldots$. . . . . . . . . . 95 
4.1 CO/Au25system. (a) Distance based features are based on the position of the dopant atoms relative to the adsorption site. (b) Hypothetical boundaries of the two nearest-neighbor layers are shown with dashed red curves. AS stands for the CO adsorbent site. Adsorbent $\mathrm{Au} / \mathrm{Ag}$ atomic sites on the surface are colored in blue. Orange is used for $\mathrm{Au} / \mathrm{Ag}$ sites in the core inaccessible for $\mathrm{CO}$ because of steric hindrance. . . . . . . . . . . . . . . . . . 103

4.2 By converting the nanocluster to a graph, we defined features that provide the learning algorithm information on the connections between atoms. . . . . . . . . . . . . . . . 105

4.3 Volume based features (a) v1 and (b) v2. . . . . . . . . . . . 106

4.4 Variation of adsorption energies . . . . . . . . . . . . . . . 109

4.5 Highest ranking important features selected by random forests and the corresponding Pearson correlation coefficient and mutual information values. $\mathrm{nAg}$ is the number of $\mathrm{Ag}$ atoms; $\mathrm{d} 3-\mathrm{d} 5$ are measures of how closely the Ag atoms are clustered around the centroid of the $\mathrm{Ag}$ locations relative to the adsorbent site; $\mathrm{HCH} 1$ is the normalized number of $\mathrm{H}-\mathrm{C}-\mathrm{H}$ fragments in the first layer of neighbors nearest to the adsorbent site; $\mathrm{d} 1$ and $\mathrm{d} 2$ are the mean and standard deviation of $\left|\mathrm{r}_{A S-A g}\right|$, and v2 is the volume enclosed by the adsorbent site and the Ag sites. . . . . . . . . . . . . . . . . 112

4.6 Features that are highly correlated with the adsorption energy according to Pearson correlation measure. . . . . . . . . . . . . . . . . . . 114

4.7 Prediction performance plots of the final model for $\mathrm{Au}_{25}$ nanocluster: (a) actual vs predicted adsorption energies and (b) histogram counts of (predicted-actual) adsorption energies. . . . . . . . . . . . . . . . . 120

4.8 Prediction performance plots for (a) $\mathrm{Au}_{36}$ and (b) $\mathrm{Au}_{133}$ nanoclusters. . . . . 124

4.9 The steric hindrance from the ligands on a spherical surface (bottom) is more than the steric hindrance from the ligands on a more planar surface (top). Color code: surface atoms, red; ligands atoms, blue, orange. 126

5.1 Equivalence between rhombohedral and hexagonal unit cells of delafossite systems. Rhombohedral unit cell is drawn with solid lines whereas the equivalent hexagonal representation is depicted using dotted lines. . . . . . . . . . . . . . . . . . . . . . . 131 
5.2 Total energy surfaces of (a) $\mathrm{AgAlO}_{2}$ and (b) $\mathrm{AgGaO}_{2}$ corresponding to different $a$ and $c$ lattice constants. . . . . . . . . . . . 133

5.3 Supercell created by repeating the 12 -atom hexagonal unit cell. . . . . . 134

5.4 Clustering factor results of (a) $\mathrm{AgAl}_{1-x} \mathrm{Fe}_{x} \mathrm{O}_{2}$ and (b) $\mathrm{AgGa}_{1-x} \mathrm{Fe}_{x} \mathrm{O}_{2}$ for $x=2 \%-5 \%$. Relative energy is defined as the difference between energy of a particular structure and the energy of the lowest energy structure for a given doping percentage $\left(\mathrm{E}-\mathrm{E}_{\min }\right) \ldots \ldots \ldots \ldots \ldots \ldots$

5.5 Layering factor results of (a) $\mathrm{AgAl}_{1-x} \mathrm{Fe}_{x} \mathrm{O}_{2}$ and (b) $\mathrm{AgGa}_{1-x} \mathrm{Fe}_{x} \mathrm{O}_{2} \quad \ldots \quad 141$

5.6 Electronic band structures of (a) $\mathrm{AgAlO}_{2}$ and (b) $\mathrm{AgGaO}_{2}$. Occupied and unoccupied bands are colored in red and blue respectively. Valence band maximum is set to $0 \mathrm{eV}$. . . . . . . . . . . . . . . 142

5.7 Average PDOS of $\mathrm{AgAl}_{1-x} \mathrm{Fe}_{x} \mathrm{O}_{2}$ (left panel) and $\mathrm{AgGa}_{1-x} \mathrm{Fe}_{x} \mathrm{O}_{2}$ (right panel $\ldots \ldots \ldots \ldots \ldots \ldots \ldots$. . . . . . . . . . . . . . . 144

5.8 Orbital DOS at the valence band edge of (a) $\mathrm{AgAlO}_{2}$ and (b) $\mathrm{AgAl}_{0.99} \mathrm{Fe}_{0.01} \mathrm{O}_{2}$ and at the conduction band region of (c) $\mathrm{AgAlO}_{2}$ and (d) $\mathrm{AgAl}_{0.99} \mathrm{Fe}_{0.01} \mathrm{O}_{2} \ldots \ldots \ldots \ldots \ldots \ldots \ldots \ldots$

5.9 Orbital DOS at the valence band edge of (a) $\mathrm{AgGaO}_{2}$ and (b) $\mathrm{AgGa}_{0.99} \mathrm{Fe}_{0.01} \mathrm{O}_{2}$ and at the conduction band region of (c) $\mathrm{AgGaO}_{2}$ and (d) $\mathrm{AgGa}_{0.99} \mathrm{Fe}_{0.01} \mathrm{O}_{2} \ldots \ldots \ldots \ldots \ldots \ldots \ldots \ldots \ldots \ldots \ldots$

5.10 PDOS in (a) correspond to a $\mathrm{AgAl}_{0.98} \mathrm{Fe}_{0.02} \mathrm{O}_{2}$ supercell where the two $\mathrm{Fe}$ atoms are separated by a distance of two nearest neighbors. The valence band edge consists of only one peak around $-3 \mathrm{eV}$ as seen in (a). However, when the Fe atoms are nearest neighbors, valence band edge is split into two main peaks about $-3 \mathrm{eV}$. . . . . . . . . . . . . . . . . . 149

5.11 Fe orbital DOS of (a) $\mathrm{AgAl}_{0.97} \mathrm{Fe}_{0.03} \mathrm{O}_{2}$ and (b) $\mathrm{AgAl}_{0.95} \mathrm{Fe}_{0.05} \mathrm{O}_{2}$ structures. The atom corresponding to the tallest Fe peak is circled in the inset . . . . . . . . . . . . . . . . . . 150

5.12 Fe orbital DOS of (a) $\mathrm{AgGa}_{0.97} \mathrm{Fe}_{0.03} \mathrm{O}_{2}$ and (b) $\mathrm{AgGa}_{0.95} \mathrm{Fe}_{0.05} \mathrm{O}_{2}$ structures. The atom corresponding to the tallest Fe peak is circled in the inset . . . . . . . . . . . . . . . . .

5.13 Unfolded band structures obtained for $\mathrm{AgAl}_{1-x} \mathrm{Fe}_{x} \mathrm{O}_{2}$ (a-c) and $\mathrm{AgGa}_{1-x} \mathrm{Fe}_{x} \mathrm{O}_{2}$ (d-f) by considering 96-atom supercells. The color scale represents the number of unfolded primitive cell bands crossing the primitive cell wave vector $k_{i}$ at energy $\epsilon_{i} \ldots \ldots \ldots \ldots \ldots$ 
5.14 Molecular orbital plots of $\mathrm{AgAl}_{1-x} \mathrm{Fe}_{x} \mathrm{O}_{2}$. (a)-(c) correspond to the valence band edge states of $0 \%, 1 \%$ and $5 \%$ Fe alloyed structures respectively. (d)-(f) correspond to the conduction band edge states of $0 \%, 1 \%$ and $5 \%$ Fe alloyed structures respectively. . . . . . . . . . . . . 156

5.15 Molecular orbital plots of $\mathrm{AgGa}_{1-x} \mathrm{Fe}_{x} \mathrm{O}_{2}$. (a)-(c) correspond to the valence band edge states of $0 \%, 1 \%$ and $5 \%$ Fe alloyed structures respectively. (d)-(f) correspond to the conduction band edge states of $0 \%, 1 \%$ and $5 \%$ Fe alloyed structures respectively. . . . . . . . . . . . . . . 157

A.1 Figures (a)-(c) depicts the Ag positions in $2^{\text {nd }}$ to $5^{\text {th }}$ lowest energy isomers for $x=6-8$. Symmetries in the unalloyed $\mathrm{Au}_{25}$ are found to be broken due to Ag atoms. . . . . . . . . . . . . . . . . . . . . . . . . 190

A.2 Structural trends in (a) 3, (b) 4, (c)5-Ag alloyed $\mathrm{Au}_{25}\left(\mathrm{SCH}_{2} \mathrm{CH}_{2} \mathrm{Ph}\right)_{18} \ldots \ldots \ldots \ldots 19 . \ldots \ldots$

A.3 Structural trends in (a) 6, (b) 7, (c)8, (d)9-Ag alloyed $\mathrm{Au}_{25}\left(\mathrm{SCH}_{2} \mathrm{CH}_{2} \mathrm{Ph}\right)_{18} \ldots \ldots \ldots \ldots$

A.4 Ag locations in (a) 19,(b) 20, (c) 21 and (d)22-Ag alloyed $\mathrm{Au}_{25}\left(\mathrm{SC}_{6} \mathrm{H}_{11}\right)_{18} \ldots \ldots \ldots \ldots \ldots$

B.1 (a) An arbitrary set of data points corresponding to feature $x$ and the (b) regression tree grown based on this data. . . . . . . . . . . . . 195

B.2 Normalized relative energies of the lowest energy isomers for alloying levels 1-12. The structure index increases with the increasing relative energy. For example, the lowest energy structure has the index of 0 and the second lowest energy structure is given in the index 1 and so on. . . . .

B.3 Normalized relative energies of the lowest energy isomers for alloying levels $13-24 . \ldots \ldots$. . . . . . . . . . . . . . . . 198

B.4 Extended feature-feature Pearson correlation map. . . . . . . . . . . . . 199

B.5 Residual error vs the actual adsorption energy for $\mathrm{Au}_{25}$. The region corresponding to the negative adsorption energies is shaded in orange.

B.6 Actual vs predicted adsorption energies of (a) $\mathrm{Au}_{36}$ and (b) $\mathrm{Au}_{133}$ nanoclusters in terms of electronvolts. . . . . . . . . . . . . . . . . . 201

B.7 Au cores of (a) $\mathrm{Au}_{36}$ and (b) $\mathrm{Au}_{133}$ nanoclusters. . . . . . . . . . . . . . 201

C.1 Fe sites in $2 \%$ Fe doped $\mathrm{AgAlFeO}_{2} \ldots \ldots$. . . . . . . . . 206 
C.2 Fe sites in $3 \%$ Fe doped $\mathrm{AgAlFeO}_{2} \ldots \ldots \ldots \ldots \ldots$. . . . . 207

C.3 Fe sites in $4 \%$ Fe doped $\mathrm{AgAlFeO}_{2} \ldots \ldots \ldots \ldots$. . . . . . 208

C.4 Fe sites in 5\% Fe doped $\mathrm{AgAlFeO}_{2} \ldots \ldots \ldots$. . . . . . . . . . 209

C.5 Fe sites in $2 \%$ Fe doped $\mathrm{AgGaFeO}_{2}$. . . . . . . . . . . . . 210

C.6 Fe sites in $3 \%$ Fe doped $\mathrm{AgGaFeO}_{2} \quad \ldots \ldots \ldots \ldots . \ldots . \ldots 211$

C.7 Fe sites in $4 \%$ Fe doped $\mathrm{AgGaFeO}_{2} \quad \ldots \ldots \ldots \ldots \ldots$

C.8 Fe sites in 5\% Fe doped $\mathrm{AgGaFeO}_{2} \ldots \ldots \ldots \ldots$

C.9 The lowest energy $8 \% \mathrm{Fe}$ doped configuration. Color code: Ag, grey; $\mathrm{Al}$ or $\mathrm{Ga}$, light blue; $\mathrm{Fe}$, orange; $\mathrm{O}$, red. . . . . . . . . . . . 215 


\section{CHAPTER I}

\section{MOTIVATION AND BACKGROUND}

Catalysis is the process of reducing the activation energy of a reaction using a substance which is known as a catalyst. As a result, catalysis can increase the reaction rate, enable reactions to take place at a lower temperature or start reactions that would otherwise be not permissible due to high energy barriers. Therefore, catalysis plays a vital role in a wide range of industrial applications. Manufacturing of almost $90 \%$ of all commercial chemical products are believed to be supported by catalysis. ${ }^{1}$ It is expected that the global market size for catalysts will reach about 34 billion US dollars by 2025 at a compound annual growth rate of $4.5 \% .^{2}$

Not surprisingly, tremendous effort has been expended over the past 30 years to produce efficient catalysts. Synthesizing mixed metal catalysts has been one of the priorities of the scientists as it provides a cost effective pathway to tune the catalytic properties to suit needs of different applications. However, studying the catalytic properties of alloyed catalysts is a challenging task both experimentally and computationally. This is mainly due to the enormity of the combinatorially possible alloyed configurations. For example, alloying a 25-atom gold nanoparticle with silver can produce over 33 million different isomers. Therefore, we need smart techniques to filter out a smaller subset of candidate structures having desired catalytic properties. Fur- 
ther, in the case of nanocatalysts, their synthesis often results in mixtures of clusters containing different number of dopants. It is not possible to understand the catalytic properties of such mixtures using a few random molecular or crystalline structures. Computational modeling of this kind of systems should take into account compositional complexities that play a significant role in determining the catalytic properties. In this thesis, we discuss novel computational approaches based on density functional theory calculations to investigate catalytic properties of alloyed catalysts. We use two types of catalytic systems to demonstrate our methods: thiolated gold nanoclusters and delafossite oxides. Thiolated gold nanoclusters have gained popularity mainly as a heterogeneous catalyst while delafossites have been tested for potential applications in photocatalysis.

\subsection{Gold nanoclusters}

Before providing a historical overview of the gold nano-systems, let me clarify some terms frequently being used in the gold-based literature: colloids, clusters, nanoclusters and nanoparticles. A cluster is defined as a group of metal atoms clustered at close proximity to each other. ${ }^{3}$ These metal atoms can bind with either other metal atoms or ligands. The chemical composition of a cluster is precisely known. Clusters can be synthesized with $0 \%$ dispersion in the size. In other words, clusters are monodispersed. In the literature, clusters are found with the number of atoms varying from 2 to 150 and they are generally synthesized in the solution phase. On the other hand, a nanoparticle identified by a given name may be composed of sev- 
eral clusters of particles having different sizes. The dispersion in the sizes of clusters in a nanoparticle is generally less than $15 \%$. The generally accepted definition of a nanoparticle is that "a particle of any shape with dimensions in the $1 \mathrm{~nm}$ to $100 \mathrm{~nm}$ range". The one given in the BSI Standards Publication 2 Nanoparticle-Vocabulary is "a particle having one or more dimensions of the order $100 \mathrm{~nm}$ or less". ${ }^{3}$ Finally, colloids are composed of several closely related clusters of which the size dispersion is greater than $15 \% .{ }^{4}$ In other words, colloids are poly-dispersed. A nano-colloid is generally identified as a colloid having number of atoms in the range $400-40000$.

The oldest evidence of gold usage can be traced back to 4,600 BC to 4,200 BC in Varna, Bulgaria where a burial site was found with gold jewelry. ${ }^{5,6}$ Based on the excellent death mask of the Egyptian pharaoh Tutankhamun (ruled 1332-1323 BC), it is reasonable to deduce that ancient craftsmen have been involved in continuous experiments with gold for a long time. ${ }^{7}$ Evidence on the soluble forms gold were found in Rome, when they discovered the Lycurgus Cup which is dated to $5^{\text {th }}$ to $4^{\text {th }}$ century BC. Experiments involving gold colloids can be dated back to medieval times. People added gold chloride to molten glass to make red stained glass in cathedrals. The belief that gold has some connection with the long life is also found in ancient Indian scriptures.

However Faraday's work on gold clusters in 1857 can be considered as a major milestone in the field of nanoparticles ${ }^{8}$. Several theoretical and experimental work on gold colloids followed, including Steubings' successful attempt to obtain optical spectra of gold ${ }^{9}$. In the same year, Gustav Mie was able to use Maxwell's equations 
produce an optical spectra which matched the one obtained by Steubing ${ }^{10}$. After the structure of DNA was resolved in early 1950s, gold nanoparticles gained popularity as a candidate for biological labels in TEM (Transmission Electron Microscope) studies of cellular structures. After Haruta et al. found that supported gold nanoparticles show catalytic properties for low temperature CO oxidation ${ }^{11}$, gold nanoparticles started to gain popularity as nano-catalysts.

\subsubsection{Thiolated gold nanoclusters}

Ligand-protected gold nanoclusters can be divided into two sub disciplines deepening on the type of ligand that stabilizes the cluster. These are phosphine and thiolate protected nanoclusters. Compared to thiolate-protected nanoclusters, the structure of phosphine based nanoclusters are simple. This is because phosphine ligands bind directly with the gold atoms whereas the thiolate ligands form staple-like structural units with gold atoms. Malatesta's work on phosphine capped gold clusters is noteworthy. ${ }^{12}$ Even though he could not determine the structures of the cluster accurately, his attempt motivated later works by Nijmegen ${ }^{13-17}$ and Mingos ${ }^{18-21}$ groups who resolved the structures of several clusters using X-ray crystallography.

Synthesis of gold particles having a thiolate coating was first reported by Brust et al. in $1994 .^{22}$ Their procedure consists of two phases in which $\mathrm{AuCl}_{4}^{-}$was reduced by $\mathrm{NaBH}_{4}$ in a medium containing alkanethiol. Works of the Whetten group in late 90's also reported thiolate-containing nanoparticles having number of atoms in the range 100 to $1300^{23}$. In 1997 , they obtained nanoparticles with around 38 
gold atoms ${ }^{24}$. Inspired by these findings Hakkinen attempted to predict the crystal structure of $\mathrm{Au}_{38}(\mathrm{SR})_{24}$ in $1999^{25}$. In 2006, Hakkinen showed for the first time that the $\mathrm{Au}$ atoms can exist in non-core sites; in $\mathrm{AuSCH}_{3}$ units surrounding the $\mathrm{Au}$ core ${ }^{26}$. The first thiolated nanocluster of which the structure was determined using X-ray crystallography is $\mathrm{Au}_{102}(\mathrm{SR})_{52}{ }^{27}$. However, what attracted the attention the most toward thiolated nanoclusters was the structure determination of $\mathrm{Au}_{25}(\mathrm{SR})_{18}$ in 2007 by Zhu et al. ${ }^{28}$ This is because its excellent stability and the ease with which DFTbased theoretical investigations can be performed to probe its properties as a result of its small size. Two methodologies to synthesize nanoclsters, namely, size focusing ${ }^{29}$ and ligand exchange induced size transformation, ${ }^{30}$ also emerged during this period which resulted in a rapid growth in the number of total structure determinations in subsequent years.

\subsubsection{Bimetallic thiolated gold nanoclusters}

With the advancements in the synthesizing techniques, experimentalists were able to create bimetallic nanoparticles. $\mathrm{Au}_{24} \mathrm{Pd}_{1}\left(\mathrm{SC}_{2} \mathrm{H}_{4} \mathrm{Ph}\right)_{18}$ can be considered as the first bimetallic thiolate protected nanocluster which was synthesized by the Murray group. ${ }^{31}$ They found that $\mathrm{Pd}$ doping changes the electro-chemical properties of $\mathrm{Au}_{25}$ significantly. Shortly afterwards in early 2010, Negishi et al. also reported the synthesis of mono $\mathrm{Pd}$ doped $\mathrm{Au}_{25}$ stabilized by $\mathrm{SC}_{12} \mathrm{H}_{25}$ ligands using high-performance liquid chromatography. ${ }^{32}$ In the same year, Negishi and co workers synthesized a series of $\mathrm{Ag}$ doped nanoclusters by altering the $\left[\mathrm{HAuCl}_{4}\right]:\left[\mathrm{AgNO}_{3}\right]$ ratio. ${ }^{33}$ In 2011 
Qian et al., doped $\mathrm{Au}_{25}$ and $\mathrm{Au}_{38}$ with $\mathrm{Pd}{ }^{34}$ By doping $\mathrm{Au}_{144}$ with approximately 30, 34, 52 and $53 \mathrm{Ag}$ atoms Kumara et al. showed that electronic properties of $\mathrm{Au}_{144}$ can be modified. ${ }^{35}$ Negishi et al. showed that doping $\mathrm{Au}_{38}\left(\mathrm{SC}_{2} \mathrm{H}_{4} \mathrm{Ph}\right)_{24}$ with two $\mathrm{Pd}$ atoms results in structures more stable than $\mathrm{Au}_{38}\left(\mathrm{SC}_{2} \mathrm{H}_{4} \mathrm{Ph}\right)_{24}$ with regard to degradation in solution and core etching by thiols. ${ }^{36}$ Qian and co-workers managed to dope $\mathrm{Au}_{25}\left(\mathrm{SC}_{2} \mathrm{H}_{4} \mathrm{Ph}\right)_{24}$ with a Pt atom. ${ }^{37}$ Using theoretical and experimental analysis they showed that Pt goes to the center of the nanocluster. They further demonstrated that the catalytic activity of $\mathrm{Au}_{24} \mathrm{Pt}_{1}\left(\mathrm{SC}_{2} \mathrm{H}_{4} \mathrm{Ph}\right)_{18}$ in the selective oxidation of styrene reaction is greater than that observed with pure $\mathrm{Au}_{25}$. Also, the HOMO-LUMO gap of $\mathrm{Au}_{24} \mathrm{Pt}_{1}\left(\mathrm{SC}_{2} \mathrm{H}_{4} \mathrm{Ph}\right)_{18}$ was found to be about $0.5 \mathrm{eV}$ less than that of $\mathrm{Au}_{25}$. In 2013, Jin group showed that $\mathrm{Au}_{25}$ can be doped by up to $4 \mathrm{Cu}$ atoms. ${ }^{38}$ In the same year, Das group managed to dope $\mathrm{Au}_{144}$ with $\mathrm{Pd}^{39}$ Later in 2013, Das group reported the synthesis and ESI-MS characterization of $\mathrm{Cu}$ doped $\mathrm{Au}_{144}{ }^{40}$ They found that the maximum number of copper atoms that can be doped in $\mathrm{Au}_{144}$ was 23. In early 2014, Kumara et al. determined the atomic positions of $\mathrm{Ag}$ atoms in $\mathrm{Au}_{25}$ doped with roughly six Ag atoms using X-ray crystallography. ${ }^{41}$ Their findings confirmed the earlier theoretical prediction that the surface of the 13-atom icosahedron is the preferable location for Ag atoms. In 2015, Kumara et al. probed the structure of $\mathrm{Ag}$ doped $\mathrm{Au}_{38}$ using X-ray crystallography. Their findings showed that Ag prefer to occupy 9 sites in the $\mathrm{Au}_{21}$ bi-icosahedral core of $\mathrm{Au}_{38}$. Later in the same year, Yao et al. compared the effects of doping $\mathrm{Au}_{25}$ with single $\mathrm{Cd}$ and $\mathrm{Hg}$ atoms. ${ }^{42}$ They found that $\mathrm{Cd}$ preferably gets doped in the $\mathrm{Au}_{13}$ icosahedron whereas $\mathrm{Hg}$ prefers sites in 
the outer shell of $\mathrm{Au}_{25}$. In 2016, marking another mile stone in heteroatom doping of thiolated nanoclusters, Jin group managed to dope Au atom in the staple units of $\mathrm{Au}_{25}{ }^{43}$

As evident from the above mentioned achievements in heteroatom doping, experimentalists have been very efficient in synthesizing and probing the structures alloyed nanoclusters. This is mainly because alloying foreign metal atoms has been proven to be an effective method to tune electronic, optical and catalytic properties of these nanoclusters. Density functional theory calculations play an important role in the efforts to understand theoretical reasons behind these experimental observations. Such understanding is essential for the discovery of novel nanoclusters having advanced properties required for future applications. In this dissertation, we focus on structural, electronic and catalytic properties of $\mathrm{Ag}$ alloyed $\mathrm{Au}_{25}(\mathrm{SR})_{18}$ nanocluster, with the hope that our findings will be used by experimental and theoretical research communities for the progress of the field of thiolated nanoclusters.

\subsection{Delafossites}

The first delafossite type discovered was $\mathrm{CuFeO}_{2}$ in 1873 by Charles Friedel, who named the material after a French mineralogist Gabriel Delafosse ${ }^{44}$. According to Marquardt et al., using a synthetically prepared sample, the crystal structure of $\mathrm{CuFeO}_{2}$ was first resolved by Soller and Thompson in $1935 .{ }^{44}$ Since then researchers have shown interest in unraveling various structural, chemical, optical and electronic properties of many types of delafossites. In 1971, a series of three papers 
of Rogers, Shannon and Prewitt reported an extensive investigation on 17 delafossite systems. ${ }^{45-47}$ In the third of these papers, they showed that broad valence and conduction band regions are due to the mixing of $s p^{3}$ hybridized O orbitals with $p_{z}$ and $1 / \sqrt{2}\left(d_{z^{2}}-s\right)$. However, the valence band edge is composed of metallic bands formed due to the mixing of $d, p_{z}$ and $1 / \sqrt{2}\left(d_{z^{2}}+s\right)$ wavefunctions of $A g^{+}$with the wavefunctions of neighboring atoms. In 1980, Garcia and co workers demonstrated the electrocatalytic properties of $\mathrm{PtCoO}_{2}, \mathrm{PdCoO}_{2}, \mathrm{PdRhO}_{2}$, and $\mathrm{PdCrO}_{2}$, using $\mathrm{O}_{2}$ reduction and $\mathrm{O}_{2}$ evolution in $\mathrm{NaOH}^{48}$ In 1984, Benko and Koffyberg reoprted on the electronic and optical properties of $\mathrm{CuAlO}_{2} \cdot{ }^{49}$ They found $\mathrm{CuAlO}_{2}$ has an allowed indirect gap of $1.65 \mathrm{eV}$ and other interband transitions with energies $2.3 \mathrm{eV}$ and $23.5 \mathrm{eV}$. Also in 1984, Monnier et al. demonstrated that $\mathrm{CuCrO}_{2}$ can catalyze $\mathrm{CH}_{3} \mathrm{OH}$ synthesis. ${ }^{50}$ In 1986, Doumerc et al. investigated magnetic properties of $\mathrm{CuCrO}_{2}, \mathrm{PdCrO}_{2}$, $\mathrm{AgCrO}_{2}, \mathrm{CuFeO}_{2}, \mathrm{CuAlO}_{2}, \mathrm{CuCoO}_{2}$ and $\mathrm{CuCoO}_{2}{ }^{51}$. Using Hall voltage and Seebeck coefficient measurements, Otabe and co workers showed that $\mathrm{AgInO}_{2}$ is a n-type semiconductor. $^{52}$ Isawa et al. reported thermoelectric power of 6 cuprate delafossites: $\mathrm{YCuO}_{2}, \mathrm{LaCuO}_{2}, \mathrm{PrCuO}_{2}, \mathrm{NdCuO}_{2}, \mathrm{SmCuO}_{2}$, and $\mathrm{EuCuO}_{2} \cdot{ }^{53}$ In 2000, Gong et al. prepared $\mathrm{CuAlO}_{2}$ films using Chemical-Vapor Deposition (CVD) method. ${ }^{54}$ The authors argue that more suitable method to produce these films is CVD as it is employed by most of the industries involved in photovoltaic devices. Later in the same year, Yanagi and co-workers also reported the preparation of $\mathrm{CuAlO}_{2}$ using pulsed laser deposition technique. ${ }^{55}$ Based on the optical transmission spectrum of $\mathrm{CuAlO}_{2}$ thin films, they found that indirect and and direct gaps of $\mathrm{CuAlO}_{2}$ are 1.8 
$\mathrm{eV}$ and $3.5 \mathrm{eV}$ respectively. The electrical conductivity of $\mathrm{CuAlO}_{2}$ was found to be $3 \times 10^{-1} \mathrm{Scm}^{-1}$ using Arrhenius plots of dc electrical conductivity. In 2001, Ueda et al. managed to grow $\mathrm{CuGaO}_{2}$ thin films on $\alpha-\mathrm{Al}_{2} \mathrm{O}_{3}$ single crystal substrates by pulsed laser deposition. ${ }^{56}$ The thin films were found to be about $80 \%$ transparent for visible light and the optical gap was found to be $3.6 \mathrm{eV}$ using the tauc plot method.

In early 2001, Yanagi and co-workers reported that doped $\mathrm{CuInO} 2$ can exhibit both p- and n-type conductivity depending on the dopant. ${ }^{57}$ Based on the sign of the Seebeck coefficient, they showed that Ca-doped $\mathrm{CuInO}_{2}$ is p-type and Sn-doped $\mathrm{CuInO}_{2}$ is n-type. Later in the same year, these authors succeeded in fabricating a transparent p-n junction using $\mathrm{CuInO}_{2}$ with a turn-on voltage of $1.8 \mathrm{eV}{ }^{58}$ In 2002 , Tate et al. tried to improve the conductivity of some delafossites using different techniques. ${ }^{59}$ Using oxygen intercalation, they managed to increase the conductivity of $\mathrm{CuScMgO}_{2}$ films from 0.001 to $20 \mathrm{~S} / \mathrm{cm}$. However, transparency of these films decreased with the increasing conductivity. In $\mathrm{CuGaFeO}_{2}$, p-type conductivity enhanced from 0.02 to $1 \mathrm{~S} / \mathrm{cm}$ after doping with Fe. The authors also synthesized Sb doped $\mathrm{CuNi}_{2} / 3 \mathrm{Sb} / 1 / 2 \mathrm{O}_{2}$ and $\mathrm{AgCo}_{2}$ and studied their transmission and conductivity. They found that the band gaps of $10 \% \mathrm{Sn}$ doped $\mathrm{CuNi}_{2} / 3 \mathrm{Sb} / 1 / 2 \mathrm{O}_{2}$ and pure $\mathrm{AgCo}_{2}$ are $3.4 \mathrm{eV}$ and $4.15 \mathrm{eV}$ respectively and that both materials have p-type conductivities. In 2003, Ataoui et al. studied magnetic properties of Fe doped $\mathrm{CuGaO}_{2}$ and $\mathrm{V}$ doped $\mathrm{CuGaO}_{2}, \mathrm{CuCrO}_{2}$ and $\mathrm{CuFeO}_{2} \cdot{ }^{60}$ In the same year Okuda et al. studied magnetic, transport and thermoelectric properties of $\mathrm{Mg}$ doped $\mathrm{CuCrO}_{2} \cdot{ }^{61} \mathrm{In}$ particular, they found that Mg doping reduces the resistivity and the Seebeck coef- 
ficient but does not affect the Neel temperature. In 2006, Vanaja et al. prepared $\alpha-\mathrm{AgGaO}_{2}$ thin films using pulsed laser deposition on $\alpha-\mathrm{AlO}_{2}$ and $\mathrm{Si}$ single crystal substrates. ${ }^{62}$ These films had a p-type electrical conductivity of $3.2 \times 10^{-4} \mathrm{Scm}-1$ at $300 \mathrm{~K}$ and $50 \%$ transparency to visible light. By drawing a tauc plot of $(\alpha h \nu)^{2}$ with respect to $h \nu$, the optical gap was estimated to be $4.12 \mathrm{eV}$. Pellicer-Porres and co-workers did a combined theory-experiment study on the electronic structure of $\mathrm{CuAlO}_{2}$ and reported that it has a indirect gap of $2.99 \mathrm{eV}$ and a direct allowed gap of $3.53 \mathrm{eV}{ }^{63}$ In 2007, Snure and Tiwari synthesized $\mathrm{CuBO}_{2}$ thin films using pulsed laser deposition. ${ }^{64}$ Their films had an indirect and direct band gaps of 2.2 and $4.5 \mathrm{eV}$. Positive Hall and Seebeck coefficients suggested p-type conductivity. In 2008, Sheets et al. did an experimental study on four Ag-based delafossites: $\mathrm{AgAlO}_{2}, \mathrm{AgGaO}_{2}$, $\mathrm{AgScO}_{2}$ and $\mathrm{AgInO}_{2} \cdot{ }^{65}$ They reported that the band gaps and visible light absorption was lower than those for powder samples of copper delafossites. In early 2009, Dong and co-workers reported on the visible light induced photocatalytic activity for the degradation of rhodamine $\mathrm{B}(\mathrm{RhB})$ and methyl orange (MO) $\mathrm{AgAlO}_{2}, \mathrm{AgGaO}_{2}$ and $\mathrm{AgInO}_{2}{ }^{66}$ The photocatalytic activity was directly proportional to the atomic radius of the B-site element and the high activty of $\mathrm{AgInO}_{2}$ was proposed to be caused by its high amounts of surface hydroxyl groups. Based on the resistivity versus temperature results of $\mathrm{CuFe}_{0.9} \mathrm{Cr}_{0.1} \mathrm{O}_{2}, \mathrm{CuCr}_{0.98} \mathrm{Mg}_{0.02} \mathrm{O}_{2}$, and $\mathrm{CuRh}_{0.9} \mathrm{Mg}_{0.1} \mathrm{O}_{2}$, Guilmeau et al. showed that the presence or absence of transition metals in the structure can affect the transport properties of these delafossites. ${ }^{67}$ Delafossites containing $\mathrm{Fe}$ and $\mathrm{Cr}$ exhibited semi-conducting character, while $\mathrm{CuRh}_{0.9} \mathrm{Mg}_{0.1} \mathrm{O}_{2}$ showed metallic behav- 
ior because its resistivity was observed to increase with the increasing temperature. Ketir et al. studied photocatalytic activity of $\mathrm{CuCrO}_{2}$ by using it to remove $\mathrm{NO}_{3}^{-} \cdot{ }^{68}$ Huda and co-workers compared electronic properties of copper delafossites composed of B-site elements in groups IIIA and IIIB. ${ }^{69}$ For this study, they considered three IIIA (Al,Ga and In) and three IIIB (Sc, Y, and La) elements. They showed that the differences between indirect and direct band gaps in group IIIA delafosites are larger than those in group IIIB delafossite and IIIA delafossites tend to crystallize in rhombohedral symmetry while IIIB delafosites prefer hexagonal symmetry.

Maignan's study on $\mathrm{Mg}$ doped $\mathrm{CuRhO}_{2}$ showed that $\mathrm{CuRh}_{1-x} \mathrm{Mg}_{x} \mathrm{O}_{2}$ undergoes a metal to insulator transition at $\mathrm{x}=0.1 .^{70}$ Singh et al. found that $\mathrm{Sn}$ dopded $\mathrm{CuInO}_{2}$ had a higher band gap by about $1.1 \mathrm{eV}$ than the undoped system. ${ }^{71}$ In late 2010, Bensaid and Russo synthesized a sereis of $\mathrm{LiCrO}_{2}$ powders with $\mathrm{Li}$ and Cr vacancies. ${ }^{72}$ The chemical formulae were determined to be $\mathrm{LiCrO}_{2}, \mathrm{LiCr}_{0.9 O} \mathrm{O}_{2}$, $\mathrm{LiCr}_{0.8} \mathrm{O}_{2}, \mathrm{LiCr}_{0.7} \mathrm{O}_{2}, \mathrm{Li}_{0.9} \mathrm{CrO}_{2}, \mathrm{Li}_{0.8} \mathrm{CrO}_{2}$ and $\mathrm{Li}_{0.7} \mathrm{CrO}_{2}$. These delafossites were tested for their catalytic activity for the combusion of soot which is an emission of diesel engines. They found that $\mathrm{LiCr}_{0.9} \mathrm{O}_{2}$ had the best soot removal capability under $350^{\circ} \mathrm{C}$. Poopanya and co-workers did a theoretical investigation on how the Seebeck coefficient $(\mathrm{S})$, electrical conductivity $(\sigma)$ and the power factor $\left(S^{2} \sigma\right)$ change with respect to different p-type acceptor levels in $\mathrm{CuAlO}_{2} \cdot{ }^{73}$ They found that the power factor is directly proportional to the energy of the acceptor level and the temperature. By using three different approximations for exchange-correlation functional, Scanlon et al. studied the electronic structure of $\mathrm{CuCrO}_{2} \cdot{ }^{74}$ They reported that PBE 
and $\mathrm{PBE}+\mathrm{U}$ underestimate and HSE06 overestimates the experimentally determined optical band gaps. They also showed that hole formation takes place at $\mathrm{Cu}$ sites. However, formation energies of $\mathrm{Mg}$ doped $\mathrm{CuCrO}_{2}$ were found to be smaller than those of the $\mathrm{CuCrO}_{2}$ with $\mathrm{Cu}$ vacancies. In early 2011, Ruttanapun and co-workers managed to synthesize $\mathrm{CuFeO}_{2}$ with $\mathrm{Pt}$ dopants in $\mathrm{Cu}$ sites. ${ }^{75}$ Measurements in the temperature range, $300 \mathrm{~K}$ - $960 \mathrm{~K}$ revealed that the Seebeck coefficient, electrical conductivity and power factor increase as the temperature and the Pt dopant percentage increase. However, the thermal conductivity was observed to decrease with the increasing temperature. Investigating on the superconducting properties of hole-doped $\mathrm{CuAlO}_{2}$, Nakanishi and Katayama-Yoshida reported that with 0.2 - 0.3 hole doping, $\mathrm{CuAlO}_{2}$ becomes a high-Tc superconductor with $\mathrm{Tc}=50 \mathrm{~K} .{ }^{76}$ Zhao et al. synthesized and studied optical, electrical and electronic properties of $\mathrm{LiYO}_{2}, \mathrm{NaYO}_{2}, \mathrm{NaYbO}_{2}$ and $\mathrm{LiYO}_{2} \cdot{ }^{77}$ The optical gaps were found to be $5.3 \mathrm{eV}\left(\mathrm{LiYO}_{2}\right), 5.4 \mathrm{eV}\left(\mathrm{NaYO}_{2}\right)$, $5.3 \mathrm{eV}\left(\mathrm{NaYbO}_{2}\right)$, and $5.2 \mathrm{eV}\left(\mathrm{LiYbO}_{2}\right)$. First principle calculations indicated that $\mathrm{NaYO}_{2}, \mathrm{NaYbO}_{2}$ and $\mathrm{LiYbO}_{2}$ have direct gaps while $\mathrm{LiYO}_{2}$ has an indirect gap. Electrical conductivity measurements showed that out of the four samples, $\mathrm{LiYO}_{2}$ had the highest conductivity of $2.210^{-6} \mathrm{Sm}^{-1}$. The application of $\mathrm{CuGaO}_{2}$ delafosites in a solar cell was reported by $\mathrm{Yu}$ and co-workers in $2012 .{ }^{78}$ They fabricated p-type dye-sensitized solar cells using $\mathrm{CuGaO}_{2}$ nanoplates. These solar cells achieved a $\mathrm{V}_{o c}$ of $357 \mathrm{mV}$ under 1 Sun AM 1.5 illumination. A study on Sn doped $\mathrm{CuFeO}_{2}$ conducted by Ruttanapun et al. revealed that the activation energy for conduction decreased as the Sn doping percentage increased. ${ }^{79}$ This study also showed that the 
optical band gap is not affected by Sn doping. A fully theoretical study of both polytypes $\left(2 \mathrm{H}\right.$ and $3 \mathrm{R}$ ) of $\mathrm{AgAlO}_{2}, \mathrm{AgGaO}_{2}$ and $\mathrm{AgInO}_{2}$ was carried out by Kumar and Persson using PBE and HSE06 exchange-correlation functionals. ${ }^{80}$ Their results showed that there was very little difference between the two polytypes in terms of the structural and electronic properties. Ruttanapun synthesized $\mathrm{CuBO}_{2}$ by solid state reaction and the optical bap of this material was found to be $3.6 \mathrm{eV}$ using a UV-VISNIR spectrometer. ${ }^{81}$ They also found that $\mathrm{CuBO}_{2}$ had activation energies of $0.147 \mathrm{eV}$ and $0.58 \mathrm{eV}$ for carrier production and electrical conduction. Han et al. studied the effects of $\mathrm{Mg}$ dopants in $\mathrm{CuCr}_{1-x} \mathrm{Mg}_{x} \mathrm{O}_{2}$, by considering the dopant percentages $0 \%$ $2 \%, 4 \%, 6 \%, 8 \%, 10 \%$, and $12 \%{ }^{82}$ One of their significant findings was that the electrical conductivity increases as the Mg percentage increases. Electrical conductivity of $12 \%$ of $\mathrm{Mg}$ doped $\mathrm{CuCrO}_{2}$ is four orders of magnitude larger than that of pure $\mathrm{CuCrO}_{2}$. In early 2014, Haycock et al. published a work on Fe doped $\mathrm{CuGaO}_{2},{ }^{83}$ in which they considered doping percentages $1 \%$ to $5 \%$ using 432 atom supercell. They reported that Fe dopants create new states near the valence band edge resulting in a reduced HOMO-LUMO gap. In late 2014, Iozzi et al. did a comparison between the electronic and transport properties of $\mathrm{Ag} \mathrm{And} \mathrm{Cu}$ based delafossites using first principle calculations. ${ }^{84}$ Based on the electronic density of states, they found that the valence band of $\mathrm{Ag}$ delafossites is broader than that of the $\mathrm{Cu}$ delafossites. Taddee and co-workers managed to synthesize Fe-doped $\mathrm{CuCrO}_{2}$ with Fe percentages varying from 0 to $15 .{ }^{85} \mathrm{X}$-ray diffraction data revealed that the Fe content did not affect the delafossite structure of doped systems. However, lattice constants were observed to 
increase as the Fe percentage was increased. The change in the optical gap $\left(\mathrm{E}_{g}\right)$ with respect to the Fe content did not exhibit a clear trend as $\mathrm{E}_{g}$ was found to be $3.61 \mathrm{eV}$ for $1 \%$ and $3 \% 10 \%$ of Fe doping. More recent works on delafossites include, discussions on optical and magnetic properties of Fe doped $\mathrm{CuGaO}_{2}$, catalytic properties of $\mathrm{Ni}$-, $\mathrm{Co}$ - and Fe-doped $\mathrm{BaCuO}_{2},{ }^{86}$ transport properties of hole doped $\mathrm{CuRhO}_{2},{ }^{87}$ and hydrogen production using $\mathrm{Fe}$ doped $\mathrm{CuCrO}_{2}$ nanopowders. ${ }^{88}$

\subsubsection{Delafossites as photocatalysts}

Despite the large optical gaps, researchers have found photocatalytic properties in several delfossite systems including $\mathrm{CuGaO}_{2}, \mathrm{CuCrO}_{2}, \mathrm{AgAlO}_{2}, \mathrm{AgGaO}_{2}, \mathrm{AgInO}_{2}$ and $\mathrm{AgFeO}_{2} \cdot{ }^{89-94}$ After Huda et al. showed using density functional theory calculations, that B-site doping can be used to tune the band structure of delafossites, ${ }^{95}$ several researches attempted to test the catalytic activity of doped delafossites. In 2011, Lekse and co-workers used $\mathrm{Fe}$ doped $\mathrm{CuGaO}_{2}$ to photoreduce $\mathrm{CO}_{2} \cdot{ }^{90}$ They noted that band gap of $\mathrm{CugaO}_{2}$ reduced from $3.75 \mathrm{eV}$ or $2.55 \mathrm{eV}$ to $1.5 \mathrm{eV}$ upon Fe doping. However, their results did not show a significant increase in the amount of $\mathrm{CO}$ produced even with $15 \% \mathrm{Fe}$ doped $\mathrm{CuGaO}_{2}$. Also, no definite pattern in the $\mathrm{CO}$ production was detected as the doping percentage was increased from $0 \%$ to $20 \%$. The same group in 2014 showed using diffuse reflectance spectroscopy measurements that the band gap of $\mathrm{NaInO}_{2}$ can be reduced from $3.9 \mathrm{eV}$ to $2.8 \mathrm{eV}$ by doping $10 \%$ of In sites with Fe. ${ }^{91}$ And the catalytic degradation of methyl blue was observed to increase under visible light due to this band gap decrease. It is surprising to find that 
no computational study has been carried out on Fe doped Ag delafossites as all the $\mathrm{AgAlO}_{2}, \mathrm{AgGaO}_{2}, \mathrm{AgInO}_{2}$ and $\mathrm{AgFeO}_{2}$ systems have been shown to exhibit catalytic properties. For example, in 2009, Dong and co-workers compared the photocatalytic activity of $\mathrm{AgAlO}_{2}, \mathrm{AgGaO}_{2}, \mathrm{AgInO}_{2}$ for rhodamine $\mathrm{B}$ and methyl orange degradation under visible light. They found that the photocatalytic activity increased as the atomic radius of the $\mathrm{B}$-site atom increased (i.e $\mathrm{AgAlO}_{2}<\mathrm{AgAlO}_{2}<\mathrm{AgInO}_{2}$ ). Yin and coworkers studied the photocatalytic activity of $\mathrm{AgFeO}_{2}$ for degradation of methyl orange. They found that $\mathrm{AgFeO}_{2}$ can degrade $97 \%$ of methyl orange during a six-hour hydrothermal procedure. The high catalytic performance was believed to be due to large surface area and high efficiency with which electron and holes separate. In this thesis, we explore the structural and electronic properties of Fe doped Ag based delafossites in an attempt to show how doping can be used to tune electronic and possibly optical properties.

Organization of this dissertation is as follows. In the first chapter, a brief overview of the FIREBALL code is given. The second chapter is mainly dedicated to a study on structural, electronic and catalytic properties of 6,7,8-Ag alloyed $\mathrm{Au}_{25}(\mathrm{SR})_{18}$ nanocluster. I also present a summary of electronic and structural properties of selected nanoclusters at the end of this chapter. In chapter three, I discuss a machine learning methodology designed to predict $\mathrm{CO}$ adsorption energies on thiolated nanoclusters. In chapter four, structural and electronic properties of Fe doped $\mathrm{AgAlO}_{2}$ and and $\mathrm{AgGaO}_{2}$ are discussed for Fe percentages from 1 to 5. Finally in chapter five I summarize the tools and methods discussed throughout this thesis. 


\section{CHAPTER II}

\section{FIREBALL}

FIREBALL is a self-consistent density functional theory (DFT) code that makes use of an ab initio tight-binding molecular dynamics simulation technique. As all the calculations in this thesis were carried out with FIREBALL, a brief theoretical description of this code is presented in this section. We use Hartree atomic units (i.e $\left.\hbar=m=e=1 /\left(4 \pi \epsilon_{0}\right)=1\right)$ to simplify the notation in all the derivations.

The fundamental problem that any DFT code aims to solve is the Schrodinger equation for a system containing many electrons and nuclei. The Hamiltonian of such a system reads,

$$
\hat{H}=\hat{T}_{n u}+\hat{V}_{n u-n u}+\hat{T}_{e l}+\hat{V}_{e l-e l}+\hat{V}_{e l-n u}
$$

Here, $\hat{T}_{n u}$ and $\hat{T}_{e l}$ are the kinetic energy operators for nuclei and electrons shown respectively as,

$$
\begin{gathered}
\hat{T}_{n u}=-\sum_{l=1}^{M} \frac{\nabla_{l}^{2}}{2 M_{l}} \\
\hat{T}_{e l}=-\sum_{i=1}^{N} \frac{\nabla_{i}^{2}}{2 m}=-\frac{1}{2} \sum_{i=1}^{N} \nabla_{i}^{2},
\end{gathered}
$$

where $\mathrm{M}$ and $\mathrm{N}$ are the number of nuclei and electrons in the system. $M_{l}$ is the mass of the nucleus $l$ and $m$ is the mass of the electron. 


$$
V_{n u-n u} \text { and } V_{e l-e l} \text { are the operators for nucleus-nucleus and electron-electron }
$$

Coulomb repulsion respectively.

$$
\begin{gathered}
\hat{V}_{n u-n u}=\frac{1}{2} \sum_{l=1, m=1}^{M} \frac{Z_{l} Z_{m}}{\left|\mathbf{R}_{\mathbf{l}}-\mathbf{R}_{\mathbf{m}}\right|} \\
\hat{V}_{e l-e l}=\frac{1}{2} \sum_{i=1, j=1}^{N} \frac{1}{\left|\mathbf{r}_{\mathbf{i}}-\mathbf{r}_{\mathbf{j}}\right|} .
\end{gathered}
$$

$V_{e l-n u}$ is the operator for Coulomb interaction between the electrons and the nuclei,

$$
\hat{V}_{e l-n u}=\sum_{i=1}^{N} v\left(\vec{r}_{i}\right),
$$

where $v\left(\vec{r}_{i}\right)=\sum_{l=1}^{M} \frac{-Z_{l}}{\left|\vec{r}_{i}-\vec{R}_{l}\right|}$. Here, $Z_{l}$ is the number of protons in each atom $l$. The wavefunction associated with the above Hamiltonian (Equation 2.1) is a function of positions of electrons and nuclei $\left(\Psi\left(\vec{r}_{i}, \vec{R}_{j}\right)\right)$. In practice, Equation 2.1 becomes progressively difficult to solve as the number of particles in a system increases. A useful approximation to reduce the computational complexity of this problem is the Born-Oppenheimer approximation. This approximation says that the electron and nuclear degrees of freedom can be separated. The justification for this approximation is based on the large disparity between the nuclear and electron masses. An electron is about 1835 times lighter than the nucleus. But the Coulomb force that each of them experiences is the same. Consequently, the velocity of the electron is much greater than that of the nucleus. As a result, any change in motion of the nucleus can be quickly matched by the fast moving electron. Therefore, we can write the many 
body wavefunction as a direct product of two wavefunctions depending only on the positions of electrons and nuclei respectively.

$$
\Psi\left(\vec{r}_{i}, \vec{R}_{j}\right)=\Psi_{e l}\left(\overrightarrow{r_{i}}\right) \otimes \Phi_{n u}\left(\vec{R}_{j}\right)
$$

Therefore, we now have two Hamiltonians to solve. The Hamiltonian which depends only on the positions of nuclei consists of $\hat{T}_{n u}$ and $\hat{V}_{n u-n u}$. Due to the slow motion of nuclei, we can neglect its kinetic energy, so that $\hat{V}_{n u-n u}$ is the only contribution from the nuclear Hamiltonian in the total energy.

The wavefunction of an N-electron system, $\Psi\left(\vec{r}_{1}, \vec{r}_{2}, \vec{r}_{3}, \ldots, \vec{r}_{N}\right)$ gives the position probability amplitude of an electron at $r_{1}$, another electron at $r_{2}, \ldots$ and the $N^{t h}$ electron at $r_{N}$. I dropped the subscript $e l$ for simplicity. From here onwards, $\Psi$ refers to the electronic wavefunction. The Schrodinger equation which dictates the dynamics of these electrons is given by,

$$
\hat{H}|\Psi\rangle=\left(\hat{T}_{e l}+\hat{V}_{e l-e l}+\hat{V}_{e l-n u}\right)|\Psi\rangle .
$$

Our goal is to solve Equation 2.2 to arrive at the energy of a system containing an interacting set of electrons. After that, we can simply add the nucleusneculeus repulsion energy term to find the total energy of the system under the Born-Oppenheimer approximation. To simplify the procedure of solving Equation 2.2, we use the first Hohenberg-Kohn theorem. According to this theorem, ground state wavefunction $\Psi_{0}(\vec{r})$ is a functional of the ground state density $\rho_{0}(\vec{r})$, 


$$
\rho_{0}(\vec{r})=N \int \Psi_{0}^{*}\left(\vec{r}, \overrightarrow{r_{2}}, \ldots . \overrightarrow{r_{N}}\right) \Psi_{0}\left(\vec{r}, \overrightarrow{r_{2}}, \ldots . \overrightarrow{r_{N}}\right) d^{3} r_{2} d^{3} r_{3} \ldots d^{3} r_{N}
$$

Therefore, any observable associated with Equation 2.2 is also a functional of $\Psi_{0}(\vec{r})$. Importantly, the ground state energy can now be written as,

$$
E_{v_{0}}=\left\langle\Psi_{0}\left|\hat{T}_{e l}+\hat{V}_{e l-e l}+\hat{V}_{e l-n u}\right| \Psi_{0}\right\rangle,
$$

where $\Psi_{0}(\vec{r})$ is the ground state wavefunction. Now, what we have to do is to find the density which minimizes the total energy of the system, subject to the constraint that the total number of electrons remains constant. In accordance with the variational technique, this minimization is given by,

$$
\begin{aligned}
\frac{\delta\left(E[\rho(\vec{r})]-\mu \int d^{3} r \rho(\vec{r})\right.}{\delta n} & =0 \\
\mu(N) & =\frac{\delta E[\rho]}{\delta \rho(\vec{r})} .
\end{aligned}
$$

Here, $\mu(N)$ is defined as the chemical potential of the system. Using the fact that electrons are indistinguishable particles, we can write $\left\langle\Psi\left|V_{e l-n u}\right| \Psi\right\rangle$ as,

$$
\begin{aligned}
\left\langle\Psi\left|V_{e l-n u}\right| \Psi\right\rangle & =\iiint \ldots \int \sum_{i=1}^{N} \Psi^{*}\left(\vec{r}_{1}, \vec{r}_{2}, \vec{r}_{3} \ldots . \vec{r}_{N}\right) v\left(\vec{r}_{i}\right) \Psi\left(\vec{r}_{1}, \vec{r}_{2}, \vec{r}_{3} \ldots . \vec{r}_{N}\right) d^{3} r_{1} d^{3} r_{2} d^{3} r_{3} \ldots . d^{3} r_{N} \\
& =N \iiint \ldots \int \Psi^{*}\left(\vec{r}_{1}, \vec{r}_{2}, \vec{r}_{3} \ldots \vec{r}_{N}\right) v\left(\vec{r}_{1}\right) \Psi\left(\vec{r}_{1}, \vec{r}_{2}, \vec{r}_{3} \ldots \vec{r}_{N}\right) d^{3} r_{1} d^{3} r_{2} d^{3} r_{3} \ldots d^{3} r_{N} \\
& =\int v(\vec{r}) \rho(\vec{r}) d^{3} r .
\end{aligned}
$$


We now have an expression for energy, with one of the terms known exactly in terms of the electron density.

$$
E=\left\langle\Psi\left|T_{e l}+V_{e l-e l}\right| \Psi\right\rangle+\int \rho(\vec{r}) v(\vec{r}) d^{3} r
$$

To obtain the energy of a system using Equation 2.3, we need to know the many-electron wavefunction $(\Psi)$. However, the form of this wavefunction is not known explicitly. Hartee-Fock, and post-Hartree-Fock (Configuration interaction, ${ }^{96,97}$ Coupled cluster ${ }^{98,99}$ Multi-configuration time-dependent Hartree ${ }^{100}$ and Quadratic configuration interaction ${ }^{101}$ ) methods expand the many-electron wavefunction as a product of single-electron wavefunctions. Such expansions imply that electrons are noninteracting which a drastic approximation. Also, these methods are computationally very expensive and using them for systems containing a large number of atoms is practically impossible. Coming up with a mapping between the interacting manyelectron system and a non-interacting system was one of the main motivations of the density functional theory. Kohn and Sham proposed a formalism which allows us to derive the total energy of an interacting system in an exact manner without making assumptions on the many-electron wavefunction. ${ }^{102,103}$ As will be discussed below, under the Kohn-Sham method, effects on the ground state energy due to electronelectron interactions are taken into account using approximations. Consequently, such approximations can result in discrepancies between the calculated physical properties and the experimental ones. However, the fact that the scientists have been successfully able to apply density functional theory based on the Kohn-Sham method to 
explain various experimental observations for over 50 years indicates that the errors due to these approximations are not very significant.

In the Kohn-Sham method, an auxiliary system is considered in which electronelectron interactions are neglected. For such a system, the single particle Schroedinger equation reads,

$$
H_{s}\left|\psi_{i}\right\rangle=\left(T_{s}+V_{s}\right)\left|\psi_{i}\right\rangle
$$

where $V_{s}$ is the potential energy operator for an electron interacting with the nuclei. Now the single particle energy can be written as,

$$
\begin{aligned}
E_{s}=\sum_{i}\left\langle\psi_{i}\left|H_{s}\right| \psi_{i}\right\rangle & =\sum_{i}\left\langle\psi_{i}\left|-\frac{1}{2} \nabla^{2}\right| \psi_{i}\right\rangle+\left\langle\psi_{i}\left|v_{s}\right| \psi_{i}\right\rangle \\
& =\sum_{i}\left\langle\psi_{i}\left|-\frac{1}{2} \nabla^{2}+v_{s}\right| \psi_{i}\right\rangle \\
& =\sum_{i} \epsilon_{i}\left\langle\psi_{i} \mid \psi_{i}\right\rangle=\sum_{i} \epsilon_{i}
\end{aligned}
$$

Here, $\psi(\vec{r})$ is the wavefunction of a single non-interacting electron. Writing the single particle kinetic energy $T_{s}$ as, $T_{s}[\rho(\vec{r})]=\sum_{i} \epsilon_{i}-\int d^{3}(r) \rho(\vec{r}) v_{s}(\vec{r})$, results in the chemical potential corresponding to the system with non-interacting electrons being equal to,

$$
\mu=\frac{\delta E_{s}[\rho(\vec{r})]}{\delta \rho(\vec{r})}=\frac{\delta T_{s}[\rho(\vec{r})]}{\delta \rho(\vec{r})}+v_{s}(r) .
$$

Now we want to add $T_{s}$ and $E_{H}$ to the Hamiltonian with electron-electron interactions. 


$$
\begin{aligned}
E[\rho(\vec{r})] & =\left(T+V_{e l-e l}\right)[\rho(\vec{r})]+V_{e l-n u}[\rho(\vec{r})] \\
& =T_{s}[\rho(\vec{r})]+E_{H}[\rho(\vec{r})]+\left(T-T_{s}+V_{e l-e l}-E_{H}\right)[\rho(\vec{r})]+\int \rho(\vec{r}) v(r) d(\overrightarrow{2}: 6)
\end{aligned}
$$

The chemical potential corresponding to Equation 2.6 reads,

$$
\begin{aligned}
\mu & =\frac{\delta E[\rho(\vec{r})]}{\delta \rho(\vec{r})}=\frac{\delta T_{S}[\rho(\vec{r})]}{\delta \rho(\vec{r})}+\frac{\delta E_{H}[\rho(\vec{r})]}{\delta \rho(\vec{r})}+\frac{\delta E_{X C}[\rho(\vec{r})]}{\delta \rho(\vec{r})}+\frac{\delta V[\rho(\vec{r})]}{\delta \rho(\vec{r})} \\
& =\frac{\delta T_{S}[\rho(\vec{r})]}{\delta \rho(\vec{r})}+V_{H}(r)+V_{X C}(r)+V(r) .
\end{aligned}
$$

In Equation 2.7, $E_{X C}=\left(T-T_{s}+V_{e l-e l}-E_{H}\right)$ is called the exchangecorrelation energy. This term consists of energy due to the exchange symmetry of the electronic wavefunctions and the influence on the motion of one electron by all the other surrounding electrons. Even though DFT is exact as a theory, (we did not make any approximations or use experimental parameters in deriving the DFT energy) form of the exchange correlation energy is not known precisely. The exchange-correlation functional is generally expressed as,

$$
E_{x c}[\rho(\vec{r})]=\int \rho\left(\overrightarrow{r^{\prime}}\right) \epsilon\left(\overrightarrow{r^{\prime}} ; \rho(\vec{r})\right) d^{3} r^{\prime}
$$

Here, $\epsilon\left(\overrightarrow{r^{\prime}} ; \rho(\vec{r})\right)$ is defined as the exchange-correlation energy per unit volume. The corresponding exchange-correlation potential is defined as,

$$
V_{x c}[\rho(\vec{r})]=\frac{\delta E_{x c}[\rho(\vec{r})]}{\delta \rho(\vec{r})}=\epsilon\left(\overrightarrow{r^{\prime}}, \rho(\vec{r})\right)+\int \rho\left(\overrightarrow{r^{\prime}}\right) \frac{\delta \epsilon_{x c}\left(\overrightarrow{r^{\prime}}\right)}{\delta \rho(\vec{r})} d^{3} r^{\prime}
$$

Several functional forms exist to approximate these exchange-correlation interactions which I briefly explain in subsection 2.0.1. 
Now compare the chemical potential for the non-interacting system (Equation 2.5) with the chemical potential for the system with electron-electron interactions (Equation 2.7). If we choose $v_{s}(r)=V_{H}(r)+V_{X C}(r)+V(r)$, our single particle density corresponds to that of the system with electron-electron interactions. In other words, when $v_{s}(r)=V_{H}(r)+V_{X C}(r)+V(r), \rho_{s}(r)$ becomes equal to $\rho(r)$.

Therefore all we have to do is to solve the Schrodinger equation for the system with non-interacting electrons with $v_{s}(r)=V_{H}(r)+V_{X C}(r)+V(r)$, and find $\psi_{i}$. We can then calculate the density $\rho_{s}(r)=\sum_{i}^{N}\left|\psi_{i}(r)\right|^{2}$, which is equal to our original density with electron-electron interactions. The total energy of the system can now be written as,

$$
\begin{aligned}
E[\rho]= & T_{s}[\rho]+E_{H}[\rho]+\left(V_{e l-e l}-E_{H}+T-T_{s}\right)[\rho]+\int v(r) \rho(r) d^{3} r \\
= & \sum_{i}^{N} \epsilon_{i}-\int v_{s}(r) \rho(r) d^{3} r+\frac{1}{2} \int v_{H}(r) \rho(r) d^{3} r+\int E_{X C}(r) \rho(r) d^{3} r+\int v(r) \rho(r) d^{3} r \\
= & \sum_{i}^{N} \epsilon_{i}-\int v_{H}(r) \rho(r) d^{3} r-\int v_{X C}(r) \rho(r) d^{3} r-\int v(r) \rho(r) d^{3} r+\frac{1}{2} \int v_{H}(r) \rho(r) d^{3} r \\
& +\int E_{X C}(r) \rho(r) d^{3} r+\int v(r) \rho(r) d^{3} r \\
= & \sum_{i}^{N} \epsilon_{i}-\frac{1}{2} \int v_{H}(r) \rho(r) d^{3} r+\int E_{X C}(r) \rho(r) d^{3} r-\int v_{X C}(r) \rho(r) d^{3} r \\
= & \sum_{i}^{N} \epsilon_{i}-E_{H}[\rho]+E_{X C}[\rho]-\int v_{X C}(r) \rho(r) d^{3} r
\end{aligned}
$$

To solve the single particle Schroedinger equation, we need a basis set to expand the single particle wavefunctions. Fireball uses slightly excited atomic orbitals as basis functions so that, 


$$
\left|\psi_{i}\right\rangle=\sum_{\mu, l} c_{i} \phi_{\mu}\left(\vec{r}-\vec{R}_{l}\right)
$$

In Equation 2.8, index $\mu$ runs over, $s, p_{x}, p_{y}$ and $p_{z}$ atomic orbitals. These orbitals, introduced by Sankey and Niklewski ${ }^{104}$ have definite cutoff radii $\left(r_{c}\right)$. The value of the wavefunction becomes exactly zero beyond the cutoff point.

This makes the orbital energy slightly higher than that of an orbital having its radius extending to infinity. However, these excitations actually happen to model the Fermi compression ${ }^{105}$ experienced by the closely packed electrons inside a solid resulting in better band structures. As a general rule of thumb, the excited energy used for each type of atom in determining the cutoff radii is 0.15 Ryd above the energy of the free atom.

The Hamiltonian $\left(H_{i, j}\right)$ and $\operatorname{overlap}\left(S_{i, j}\right)$ matrix elements are calculated using a numerical grid. The matrix elements becomes exactly zero, if sum of the cutoff $\operatorname{radii}\left(r_{c}\right)$ corresponding to orbitals $i$ and $j$ is less than the distance separating the centers of the orbitals. ${ }^{104,106}$ For example in the system depicted in Figure 2.1, the matrix element $\left\langle\Psi_{a}|H| \Psi_{b}\right\rangle$ is zero if $\left|\mathbf{R}_{a}-\mathbf{R}_{b}\right|>r_{c, a}+r_{c, b}$. 


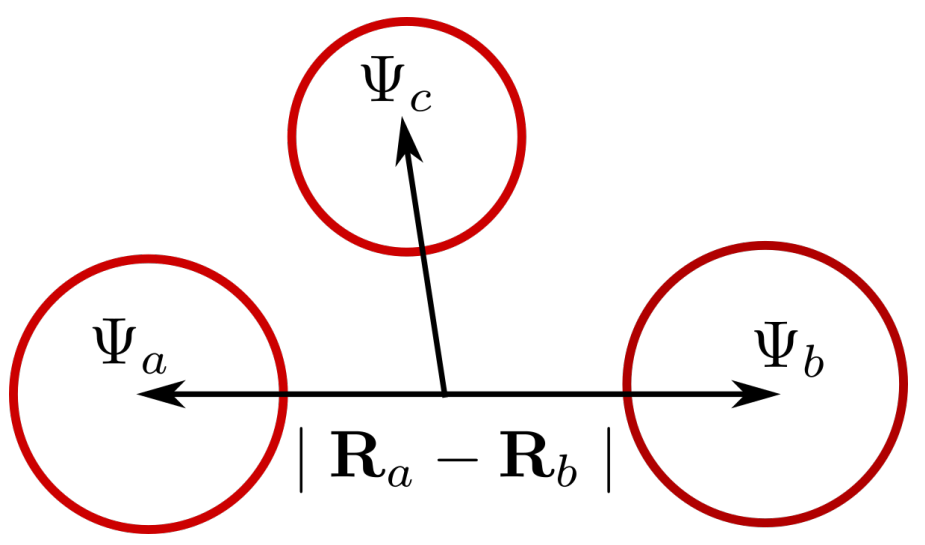

Figure 2.1: A system with three centers. The matrix elements are zero if $\left|\mathbf{R}_{i}-\mathbf{R}_{j}\right|>$ $r_{c, i}+r_{c, j}$ corresponding to any orbital $i$ and $j$.

One, two and three center interactions between the atomic orbitals are exactly evaluated using the above mentioned radial cut-off radii and stored in data files to be read during the simulation.

Arriving at the ground state energy of a given system requires solving the single particle Schroedinger equation many times as the density corresponding to this energy is not known initially. Therefore, one has to start with a guessed initial density. In Fireball, this density is set equal to the sum of neutral atom densities. Using this density, Equation 2.4 is solved to find the wavefunctions. At this point, we have to check whether our current solutions are self consistent. In Fireball, this is done by defining the output density as, $\rho_{i}^{\text {out }}=2 \sum_{\alpha}\left|\left\langle\psi_{\alpha} \mid \phi_{i}\right\rangle\right|^{2}$. ${ }^{107}$ Here, $\alpha$ runs over all the occupied states. The current solutions are self consistent if $\rho_{i}^{\text {out }}=\rho_{i}$ for all the electrons $(i=1$ to $N)$.

Once we find the self consistent total energy $\mathrm{E}_{t o t}$, of the the system, the force 
$\mathbf{F}_{i}$ acting on each atom $i$ at position $\mathbf{R}_{i}$ are calculated according to,

$$
\mathbf{F}_{i}=\frac{\delta E_{t o t}}{\delta \mathbf{R}_{i}}
$$

The new positions of the atoms can then be updated using Newton's $2^{\text {nd }}$ Law.

\subsubsection{Approximations to exchange-correlation function}

FIREBALL currently facilitates the use of two approximated functionals to obtain exchange correlation energy: LDA and B3LYP. ${ }^{108,109}$ LDA, which stands for local density approximation, calculates the exchange correlation energy at position $\vec{r}$ by using the electron density at $\vec{r}$ of a homogeneous electron gas (HEG), so that,

$$
\begin{aligned}
\epsilon_{x c}(\vec{r}) & =\epsilon_{x c}^{H E G}(\rho(\vec{r})) \\
& =\epsilon_{x}^{H E G}(\rho(\vec{r}))+\epsilon_{c}^{H E G}(\rho(\vec{r})) .
\end{aligned}
$$

In Equation 2.9, $\epsilon_{x}$ and $\epsilon_{c}$ represent contributions from exchange and correlation energies respectively. B3LYP (Becke, three-parameter, Lee-Yang-Parr) exchange correlation energy is constructed by burrowing contributions from exchange and correlation energies in LDA, GGA (generalized gradient approximation) ${ }^{108,110,111}$ and Hartree Fock (HF) formalisms. This functional is given by,

$$
E_{x c}^{B 3 L Y P}=E_{x}^{L D A}+a_{0}\left(E_{x}^{H F}-E_{x}^{L D A}\right)+a_{x}\left(E_{x}^{G G A}-E_{x}^{L D A}\right)+E_{c}^{L D A}+a_{c}\left(E_{c}^{G G A}-E_{c}^{L D A}\right),
$$

where $a_{0}=0.20 \quad a_{x}=0.72, a_{c}=0.81$. 
In FIREBALL exchange-correlation functional is expanded in two ways. The first method is called the Horsfiled approximation which is appropriate for molecules and clusters of atoms. Expansion of the matrix elements depends on the positions around which the orbitals are centered. Consider two atomic orbitals $\mu$ and $\nu$ centered at atoms $i$ and $j$ respectively. If both orbitals are on the same atom (so that $i=$ $j \equiv i$ ), the matrix elements for exchange-correlation potential is given by,

$$
\left\langle\mu\left|V_{x c}[\rho]\right| \nu\right\rangle \bumpeq\left\langle\mu\left|V_{x c}\left[\rho_{i}\right]\right| \nu\right\rangle+\sum_{j \neq i}\left\langle\mu\left|V_{x c}\left[\rho_{i}+\rho_{j}\right]-V_{x c}\left[\rho_{i}\right]\right| \nu\right\rangle
$$

On the other hand, if the two orbitals are centered at different atoms, that is, $i \neq j$, the matrix elements can be expressed as,

$$
\left\langle\mu\left|V_{x c}[\rho]\right| \nu\right\rangle=\left\langle\mu\left|V_{x c}\left[\rho_{i}+\rho_{j}\right]\right| \nu\right\rangle+\sum_{k \neq i, j}\left\langle\mu\left|V_{x c}\left[\rho_{i}+\rho_{j}+\rho_{k}\right]-V_{x c}\left[\rho_{i}+\rho_{j}\right]\right| \nu\right\rangle
$$

The second approach, which is called the McWEDA approximation is more appropriate for periodic crystals. In this expansion, spherically symmetric orbitals are used to define the average densities defined as,

$$
\bar{\rho}_{\mu \nu}=\frac{\left\langle\phi_{\mu}|\rho| \phi_{\nu}\right\rangle}{\left\langle\phi_{\mu} \mid \phi_{\nu}\right\rangle}
$$

Using this average density, the on-site $(i=j)$ matrix elements are given by

$$
\begin{aligned}
\left\langle\mu\left|V_{x c}[\rho]\right| \nu\right\rangle & \simeq\left\langle\mu\left|V_{x c}\left[\rho_{i}\right]\right| \nu\right\rangle \\
& +V_{x c}\left[\bar{\rho}_{\mu \nu}\right]\langle\mu \mid \nu\rangle+V_{x c}^{\prime}\left[\bar{\rho}_{\mu \nu}\right]\left(\langle\mu|\rho| \nu\rangle-\bar{\rho}_{\mu \nu}\langle\mu \mid \nu\rangle\right) \\
& -V_{x c}\left[\bar{\rho}_{i}\right]\langle\mu \mid \nu\rangle-V_{x c}^{\prime}\left[\bar{\rho}_{i}\right]\left(\left\langle\mu\left|\rho_{i}\right| \nu\right\rangle-\bar{\rho}_{i}\langle\mu \mid \nu\rangle\right)
\end{aligned}
$$


whereas the off-site $(i \neq j)$ matrix elements take the form (Equations 2.12),

$$
\begin{aligned}
\left\langle\mu\left|V_{x c}[\rho]\right| \nu\right\rangle & =\left\langle\mu\left|V_{x c}\left[\rho_{i}+\rho_{j}\right]\right| \nu\right\rangle+\left(\left\langle\mu\left|V_{x c}[\rho]\right| \nu\right\rangle-\left\langle\mu\left|V_{x c}\left[\rho_{i}+\rho_{j}\right]\right| \nu\right\rangle\right) \\
& \simeq\left\langle\mu\left|V_{x c}\left[\rho_{i}+\rho_{j}\right]\right| \nu\right\rangle \\
& +V_{x c}\left[\bar{\rho}_{\mu \nu}\right]\langle\mu \mid \nu\rangle+V_{x c}^{\prime}\left[\bar{\rho}_{\mu \nu}\right]\left(\langle\mu|\rho| \nu\rangle-\bar{\rho}_{\mu \nu}\langle\mu \mid \nu\rangle\right) \\
& -V_{x c}\left[\bar{\rho}_{i j}\right]\langle\mu \mid \nu\rangle-V_{x c}^{\prime}\left[\bar{\rho}_{\mu \nu}\right]\left(\left\langle\mu\left|\rho_{i}+\rho_{j}\right| \nu\right\rangle-\bar{\rho}_{\mu \nu}\langle\mu \mid \nu\rangle\right) .
\end{aligned}
$$

\subsubsection{Pseudopotentials}

Wavefunctions of electrons close to the nucleus display rapid oscillations. Computational modeling of this behavior is very expensive. Luckily, electrons close to nucleus (or core electrons) have little influence on the valence electrons. This allows us to decouple the core states and valence states. In the pseudopotential scheme, core states are replaced by a smooth function without any oscillations near the core region. This modified wavefunction in turn results in a potential, which is called the pseudopotential. This potential is different from the Coulomb potential. Now the effective potential experienced by the single particle states is evaluated only for the valence electrons using the valence electron density. The potential due to the nuclei is replaced by the pseudopotential.

$$
v_{s}\left(\rho_{v a l}\right)=V_{e x t}^{P P}+V_{H}\left(\rho_{v a l}\right)+V_{x c}\left(\rho_{v a l}\right)
$$


In FIREBALL, norm-conserving pseudopotentials are generated following the approaches detailed in papers of Hamann ${ }^{112}$ or Troullier and Martins. ${ }^{113}$ When constructing pseudopotentials the first step is to solve the single-particle Schrodinger equation for all the valence and core electrons. Next, we have to decide a cut-off radial distance $\left(r_{c}\right)$, beyond which is considered as the valence region. This step has to be performed carefully as the bonding between atoms is sensitive to the nature of the valence states. The region from the origin to $r_{c}$ is identified as the core. The wavefunction corresponding to the core is replaced by a smooth function of which the first and the second derivatives with respective position are continuous at $r_{c}$. This pseudo-wavefunction (smooth core function + valence wavefunction) can be used in the single-particle Schrodinger equation to obtain the corresponding pseudo-potential. 


\section{CHAPTER III}

\section{STRUCTURAL,ELECTRONIC AND CATALYTIC PROPERTIES OF AG \\ ALLOYED AU 25 NANOCLUSTER}

\subsection{Introduction}

Thiolate-protected gold nanoclusters $\mathrm{Au}_{\mathrm{m}}(\mathrm{SR})_{\mathrm{n}}$, have attracted scientific attention as novel functional nanomaterials due to their excellent stability ${ }^{28,114-118}$ and unique physical and chemical properties that distinguish them from their bulk counterpart ${ }^{119-122}$. Synthesizing nanoclusters precisely at the atomic level provides a platform to study the fundamental properties of these clusters in depth ${ }^{123} . \mathrm{Au}_{25}(\mathrm{SR})_{18}$ is one of the widely studied gold nanoclusters that exhibits sizes smaller than $2 \mathrm{~nm}^{38,42,124-127}$. This nanoparticle has a core-shell structure (see Figure 3.1); the core is composed of an $\mathrm{Au}_{13}$ icosahedron surrounded by $12 \mathrm{Au}$ atoms held in staples of SR-Au-SR-Au-SR units, with SR denoting the thiolate ligand ${ }^{28,114,128,129}$. The total $25 \mathrm{Au}$ atoms are divided into three categories: the center $\mathrm{Au}$ atom, $12 \mathrm{Au}$ atoms closest to the center atom (which will hereafter be called the surface of the $\mathrm{Au}_{13}$ icosahedron), and the outer shell of remaining $12 \mathrm{Au}$ atoms interacting with the ligands. All of the $\mathrm{Au}$ atoms in the surface of the $\mathrm{Au}_{13}$ icosahedron and the outer shell are bonded to thiolate ligands. Given this unique structural configuration, the molecular behavior of 
$\mathrm{Au}_{25}(\mathrm{SR})_{18}$ opens up many possibilities to alter the electronic properties considerably by substituting heteroatoms ${ }^{37,130-132}$.
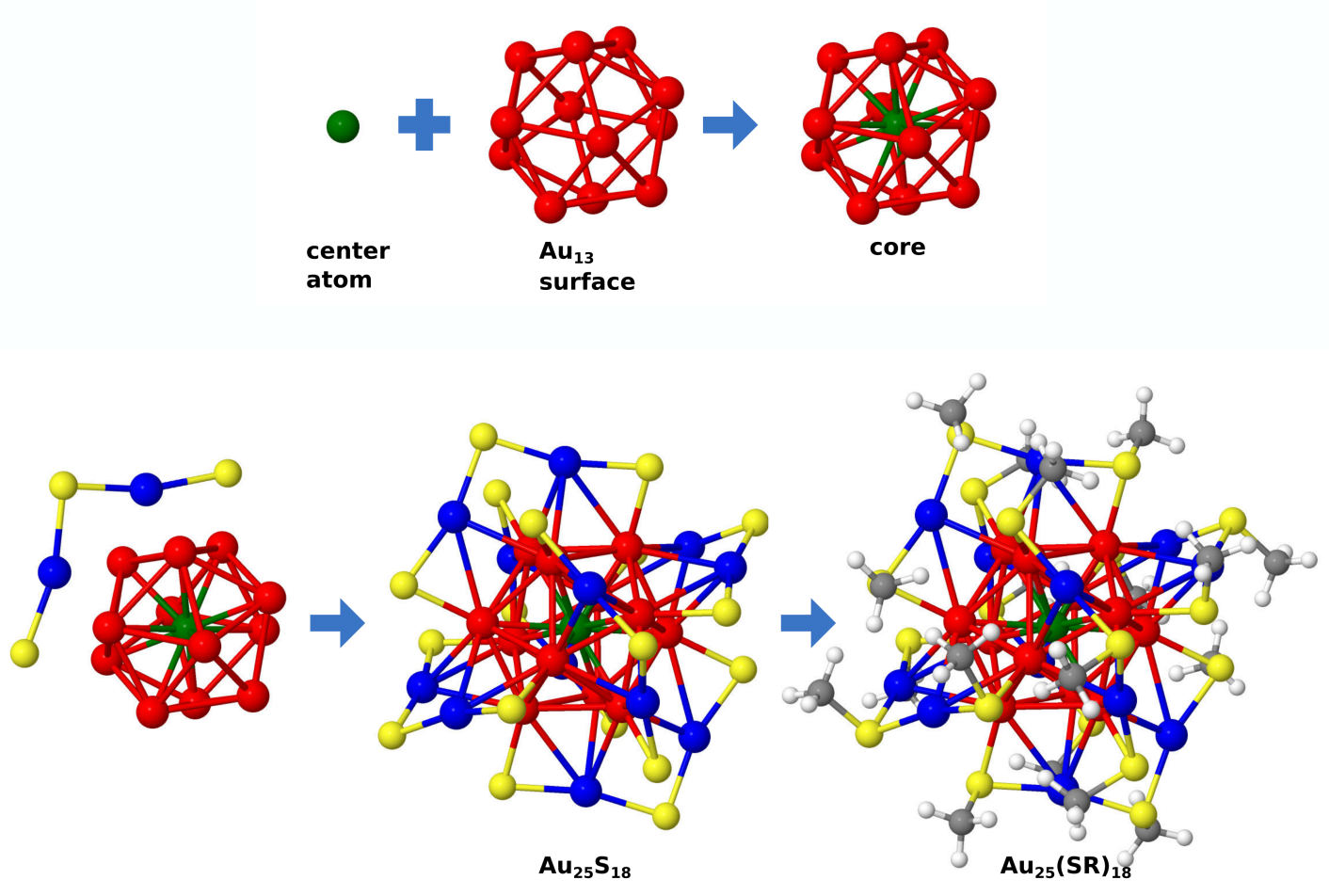

Figure 3.1: Structure of $\mathrm{Au}_{25}$ nanocluster consists of a 13-atom core surrounded by a surface layer of SR-Au-SR-Au-SR staple units. Every Au atom in the staples is bonded with a $\mathrm{Au}$ atom in the core. Color code: $\mathrm{Au}$ in the core, green and red; $\mathrm{Au}$ on the surface, blue; S, yellow; C, gray; H, off white.

A single substitution of a heteroatom can significantly change the electronic structure and chemical properties in $\mathrm{Au}_{25}(\mathrm{SR})_{18} 31,130,133,134$. Jiang and Dai have proposed 16 elements as candidates for doping the $\mathrm{Au}_{25}(\mathrm{SR})_{18}$ cluster such that the 
electronic and the geometric structure of the unalloyed system are maintained ${ }^{133}$. Several groups have been successful in increasing the number of dopants in the cluster $^{33,38}$. These reports show that the maximum $\mathrm{Ag}$ incorporation can be up to 12 atoms.

A study by Walter and Moseler proposed that the preferential occupying positions of $\mathrm{Ag}$ should occur in the $\mathrm{Au}_{13}$ icosahedran core ${ }^{135}$. This result was also confirmed by later studies of Guidez et al., Gottlieb et al. and Tlahuice-Flores ${ }^{38,115,136}$. A study by Kumara et al. showed using X-ray crystallographic data, that the central $\mathrm{Au}$ atom of $\mathrm{Au}_{13}$ core is exclusively an $\mathrm{Au}$ domain, while the remainder of the $\mathrm{Au}_{13}$ is partially occupied by $\mathrm{Au}$ or $\mathrm{Ag}$ atoms ${ }^{41}$. Recent work by Jin et al demonstrated that it is also possible to dope the staple sites by increasing the number of Ag atoms up to $19^{43}$.

A study by Negishi et al. found that the electronic structure of $(\mathrm{AuAg})_{25}(\mathrm{SR})_{18}$ can be continuously modulated by the incorporation of up to $11 \mathrm{Ag}$ atoms ${ }^{33}$. They demonstrated that alloying $\mathrm{Ag}$ was possibly an effective method to tune the electronic structure and physical properties. Kauffman et al. have investigated $\mathrm{Au}_{25-x} \mathrm{Ag}_{x}(\mathrm{SR})_{18}$, where $\mathrm{x}=0$ to 5 and $\mathrm{R}=\mathrm{C}_{2} \mathrm{H}_{4} \mathrm{Ph}$, combining both experimental and computational studies $^{137}$. They have observed that mixing $\mathrm{Au}$ and $\mathrm{Ag}$ electronic states perturbs discrete energy levels, which leads to a shift of the optical absorption peak and introduces new features into the $\mathrm{Au}_{25-x} \mathrm{Ag}_{x}$ absorption spectrum. Kumara et al. have also shown that the optical absorption peaks shift to higher energies in $\mathrm{Au}_{25}$ nanocluster due to $\mathrm{Ag}$ alloying ${ }^{41}$. This result has been reproduced computationally by Tlahuice- 
Flores who further showed that the peak corresponding to the HOMO-LUMO gap shifts to lower energies in alloyed clusters that have greater than $12 \mathrm{Ag}$ atoms ${ }^{136}$. Several groups report on the catalytic activity of the pure $\mathrm{Au}_{25}(\mathrm{SR})_{18}$ system ${ }^{138-141}$. Jin et al. have found that $\mathrm{Au}_{3}$ sites in $\mathrm{Au}_{25}(\mathrm{SR})_{18}$ are the most reactive and demonstrated that with $\mathrm{R}=\mathrm{CH}_{2} \mathrm{CH}_{2} \mathrm{Ph}$, this nanocluster supported on oxides can catalyze Sonogashira cross-coupling reaction between phenylacetylene and p-iodoanisole with high conversion of p-iodoanisole ${ }^{138,142}$. Using DFT methods, Jiang et al. have presented a possible mechanism for the selective hydrogenation of $\alpha, \beta$-unsaturated ketones to unsaturated alcohols on the $\mathrm{Au}_{25}(\mathrm{SR})_{18}$ cluster $^{140}$. Li and Jin have investigated the catalytic properties of $\mathrm{Ag}_{x} \mathrm{Au}_{25-x}(\mathrm{SR})_{18}$ and $\mathrm{Cu}_{x} \mathrm{Au}_{25-x}(\mathrm{SR})_{18}$ based on the selective oxidation of styrene to styrene epoxide and benzaldihyde ${ }^{143}$. They have found that the inner shell doped with $\mathrm{Cu}$ or $\mathrm{Ag}$ affects the selectivities for the main products. $\mathrm{Li}$ et al. have compared the catalytic activity of titania-supported $\mathrm{Au}_{25}(\mathrm{SR})_{18}$ and $\mathrm{M}_{x} \mathrm{Au}_{25-x}(\mathrm{SR})_{18}(\mathrm{M}=\mathrm{Pt}, \mathrm{Cu}, \mathrm{Ag})$ and found that $\mathrm{Au}_{25}(\mathrm{SR})_{18}$ performed better than the other bimetalic clusters ${ }^{144}$. The catalytic activity of $\mathrm{M}_{x} \mathrm{Au}(\mathrm{M}=\mathrm{Au}, \mathrm{Cu}, \mathrm{Ag})$ supported on $\mathrm{CeO}_{2}$ has been shown to decrease in the order $\mathrm{Cu}_{x} \mathrm{Au}_{25-x}\left(\mathrm{SC}_{2} \mathrm{H}_{4} \mathrm{Ph}\right)_{18}>$ $\mathrm{Au}_{25}\left(\mathrm{SC}_{2} \mathrm{H}_{4} \mathrm{Ph}\right)_{18}>\mathrm{Ag}_{x} \mathrm{Au}_{25-x}\left(\mathrm{SC}_{2} \mathrm{H}_{4} \mathrm{Ph}\right)_{18}$ by Li et al. based on the $\mathrm{CO}$ conversion to $\mathrm{CO}_{2}$. Their DFT calculations indicate that adsorption energy of $\mathrm{CO}$ on the nanocluster follow the same trend as that of the reactivity ${ }^{145}$. Even though the size of $\mathrm{Au}_{25}$ nanocluster is quite accessible for the general DFT computational techniques, it is still challenging to determine the doping sites and geometric structures once the doping number increases beyond $\mathrm{x}=4$. 
Although experimental work has mapped out the preferred Ag doping sites to be on the icosahedral shell, the partial occupancy in X-ray analysis only gave an averaged result, which did not tell the specific configuration of dopants; for example, whether the Ag dopants would be clustering together or maximally isolated.

Herein, we combine both experimental and computational research to study structural, electronic, and catalytic properties of $\mathrm{Ag}$ substituted $\operatorname{Au}_{25-\mathrm{x}} \mathrm{Ag}_{\mathrm{x}}(\mathrm{SR})_{18}$ cluster with $\mathrm{x}=6,7,8$.

\subsubsection{Computational and theoretical Tools}

The starting point of our computational procedure is to choose an optimal $\mathrm{Au}_{25}(\mathrm{SR})_{18}$ structure that possesses the geometric and chemical features similar to that of the actual experimentally synthesized $\mathrm{Au}_{25}\left(\mathrm{SC}_{2} \mathrm{H}_{4} \mathrm{Ph}\right)_{18}$ nanocluster. We adopted the geometry of $\mathrm{Au}_{25} \mathrm{SC}_{2} \mathrm{H}_{4} \mathrm{Ph}$ and replaced the $-\mathrm{SC}_{2} \mathrm{H}_{4} \mathrm{Ph}$ with $-\mathrm{SCH}_{3}{ }^{28}$.

All the calculations were carried out using FIREBALL, an open source DFT package which is based on the density functional theory, implemented within a nonlocal pseudo-potential scheme ${ }^{106,146}$. Two types of exchange-correlation density functionals are available with FIREBALL, LDA and GGA (BLYP) ${ }^{106,147}$. Within the LDA, the exchange-correlation is designed to reproduce the energy and potential of the uniform electronic structures. On the other hand, the Becke exchange with LeeYang-Parr correlation functional (BLYP) provides reasonably good energy values for metal containing systems and high levels of accuracy in the determination of a ge-

ometry optimization $^{148}$. In our study, the geometric structures and energies are of 
primary focus. Thus, the BLYP exchange was used. As for the basis set, we chose the optimized numerical local atomic orbitals. These orbital wavefunctions have a cutoff radii $R_{c}$, beyond which the wavefunctions vanish. The single numerical (SN) basis set was selected for $\mathrm{H}$ with $\mathrm{R}_{\mathrm{c}}$ values, $\mathrm{R}_{c}^{H}(s)=4.1$ a.u. and the double numerical $(\mathrm{DN})$ basis set was selected for $\mathrm{C}, \mathrm{O}$ and $\mathrm{S}$ with $\mathrm{R}_{\mathrm{c}}$ values, $\mathrm{R}_{c}^{H}(s)=4.3$ a.u, $\mathrm{R}_{c}^{H}(p)$ $=4.7$ a.u, $\mathrm{R}_{c}^{O}(s)=3.6$ a.u, $\mathrm{R}_{c}^{O}(p)=4.0$ a.u, $\mathrm{R}_{c}^{S}(s)=4.5$ a.u, $\mathrm{R}_{c}^{S}(p)=5.0$ a.u. For the metal elements $(\mathrm{Ag}$ and $\mathrm{Au})$, we used the polarized basis set $\mathrm{sp}^{3} \mathrm{~d}^{5}$ by adding a $\mathrm{p}$ orbital to the minimal basis set $\mathrm{sd}^{5}$. The cutoff radii were $\mathrm{R}_{c}^{A g}(s)=5.6$ a.u, $\mathrm{R}_{c}^{A g}(p)=$ 5.9 a.u, $\mathrm{R}_{c}^{A g}(d)=4.9$ a.u; $\mathrm{R}_{c}^{A u}(s)=5.4$ a.u, $\mathbf{R}_{c}^{A u}(p)=5.8$ a.u, $\mathrm{R}_{c}^{A u}(d)=5.0$ a.u.

Fukui functions

To explain the trends in the catalytic properties of $\mathrm{Au}_{25}(\mathrm{SR})_{18}$ clusters, we used Fukui functions as the primary reactivity descriptor ${ }^{149,150}$. Fukui functions are defined as the change in electron density when an electron is added or removed from the ground state system.

$$
f(\vec{r})=\left(\frac{\partial \rho(\vec{r})}{\partial N}\right)_{v(\vec{r})}
$$

As there are two ways to change the number of electrons in the system (by adding or removing), two main kinds of Fukui functions exist. These are electrophilic $\left(f^{-}(\vec{r})\right)$ and nucleophilic $\left(f^{+}(\vec{r})\right)$ attack Fukui functions, which are defined as

$$
\begin{aligned}
& f^{+}(\vec{r}) \equiv \rho_{N 0+1}(\vec{r})-\rho_{N 0}(\vec{r}) \\
& f^{-}(\vec{r}) \equiv \rho_{N 0}(\vec{r})-\rho_{N 0-1}(\vec{r}) .
\end{aligned}
$$


Here, $\rho_{N 0}(\vec{r}), \rho_{N 0+1}(\vec{r})$ and $\rho_{N 0-1}(\vec{r})$ are the electron densities of the ground state, one electron added and one electron removed systems respectively. If a particular site prefers to donate electrons, we expect to see a larger drop in the electron density at that site when an electron is taken out of the system. For such sites, $\rho_{N 0-1}(\vec{r}) \ll$ $\rho_{N 0}(\vec{r})$. Therefore, the larger the $f^{-}$, the greater is the tendency to donate electrons. Analogously, the sites with large $f^{+}(\vec{r})$ prefer to attract electrons.

A third form of the Fukui function is also defined as the average of electrophilic and nucleophilic attack Fukui functions; $0.5 \times\left(f^{+}(\vec{r})+f^{-}(\vec{r})\right)$. This function is called the radical attack Fukui function. Using $f^{+}(\vec{r})$ and $f^{-}(\vec{r})$ we can define the dual descriptor $(\Delta f(\vec{r}))$ as,

$$
\Delta(f(\vec{r}))=f^{+}(\vec{r})-f^{-}(\vec{r})
$$

which contains information about both the electrophilic and nucleophilic nature of a given site. If $\Delta f(\vec{r})>0$, then $f^{+}(\vec{r})$ is greater than $f^{-}(\vec{r})$, indicating that the corresponding molecular site at position $\vec{r}$ prefers to attract electrons. On the other hand a negative $\Delta f(\vec{r})$ indicates a site preferring to donate electrons.

As Fukui functions are functions of position, generating a visualization showing Fukui values at each spatial grid point is the main way to extract information on reactivity. This makes it difficult to carry out a quantitative analysis on the reactive sites of a given molecule. The situation worsens when we have to study the catalytic properties many systems, as it is the case in our current study. To overcome these 
inconveniences, condesned-to-atom Fukui functions have been defined such that for any atom $k$,

$$
f_{k}^{+}=q_{k}(N 0+1)-q_{k}(N 0)
$$

and

$$
f_{k}^{-}=q_{k}(N 0)-q_{k}(N 0-1),
$$

where $q_{k}$ is the charge attributed to atom $k$. Evaluating these functions for a system with $N$ electrons involves finding atomic charges corresponding to $N-,(N-1)$ , and $(N+1)$-electron systems. For example, consider $g^{k}(\vec{r})$ as the fraction of the total electron density $(\rho(\vec{r}))$ that can be attributed to atom $k$. The Fukui functions in terms of $g^{i}(\vec{r})$ can be expressed as,

$$
\begin{aligned}
f^{ \pm}(\vec{r}) & =\left(\frac{\partial \sum_{k} g^{k}(\vec{r}) \rho(\vec{r})}{\partial N}\right)_{v(\vec{r})}^{ \pm} \\
& =\sum_{k}\left(\frac{\partial g^{k}(\vec{r}) \rho(\vec{r})}{\partial N}\right)_{v(\vec{r})}^{ \pm} \\
& =\sum_{k}\left(\frac{\partial \rho^{k}(\vec{r})}{\partial N}\right)_{v(\vec{r})}^{ \pm},
\end{aligned}
$$

where $\rho^{k}(\vec{r})$ is the electron density associated with atom $k$. Now to obtain Fukui functions for each atom, we can integrated $\partial \rho^{k}(\vec{r}) / \partial N$ over the volume associated with atom $k$.

Consequently, the condensed-to-atom dual descriptor is given by,

$$
\Delta f_{k}=f_{k}^{+}-f_{k}^{-} .
$$


Several method exists to evaluate the atomic populations $q_{k}$. These include Mulliken, ${ }^{151}$ Natural Population Analysis, ${ }^{152}$ Hirshfeld, ${ }^{153}$ Bader ${ }^{154}$ and Löwdin ${ }^{155}$. FIREBALL implements Mulliken, Natural Population and Löwdin techniques. For our calculations we used Löwdin population analysis was considered.

An alternate method to directly obtain condensed Fukui functions was proposed by Péreza et al. This derivation is based on the assumption that changes in the electron density due to the changes in the number of electrons takes place only in the frontier molecular orbitals ${ }^{156,157}$. Thus, $f^{+}$and $f^{-}$can be approximated as

$$
\begin{aligned}
& f^{+}(\vec{r}) \approx \rho_{L U M O}(\vec{r}) \\
& f^{-}(\vec{r}) \approx \rho_{H O M O}(\vec{r})
\end{aligned}
$$

Because a particular molecular orbital $\Phi^{\alpha}(\vec{r})$ can be expanded using the basis functions, we have,

$$
f^{\alpha}(\vec{r})=\left|\Phi^{\alpha}(\vec{r})\right|^{2}=\sum_{i} \sum_{j} c_{i, \alpha} c_{j, \alpha}^{*} \phi_{i}(\vec{r}) \phi_{j}(\vec{r})^{*}
$$

Using the fact that the molecular orbitals are normalized, we get, 


$$
\begin{aligned}
\int d^{3} r f^{\alpha}(\vec{r}) & =\int d^{3} r \sum_{i} \sum_{j} c_{i, \alpha} c_{j, \alpha}^{*} \phi_{i}(\vec{r}) \phi_{j}(\vec{r})^{*} \\
& =\sum_{i}\left|c_{i, \alpha}\right|^{2} \int d^{3} r \phi_{i}(\vec{r}) \phi_{j}(\vec{r})^{*}+\sum_{i} \sum_{j} c_{i, \alpha} c_{j, \alpha}^{*} \int d^{3} r \phi_{i}(\vec{r}) \phi_{j}(\vec{r})^{*} \\
& =\sum_{i}\left|c_{i, \alpha}\right|^{2}+\sum_{i} \sum_{j} c_{i, \alpha} c_{j, \alpha}^{*} S_{i, j} \\
& =\sum_{i}\left(\left|c_{i, \alpha}\right|^{2}+\sum_{j} c_{i, \alpha} c_{j, \alpha}^{*} S_{i, j}\right)=1
\end{aligned}
$$

The quantity inside the parenthesis in the last line of Equation 3.3 can be identified as the orbital component of the Fukui function $f_{i}^{\alpha}$. By summing all the orbital components belonging to a particular atom, we can finally obtain the Fukui function condensed to an atom $k, f_{k}^{\alpha}$ as

$$
f_{k}^{\alpha}=\sum_{i \in k} f_{i}^{\alpha}
$$

We have implemented this method to obtain condensed Fukui functions in FIREBALL. As pointed out by some researchers, this method suffer from some deficiencies, thus was not used in our calculations. ${ }^{158}$ However, Equation 3.4 can be used to estimate the HOMO and LUMO charge associated with atomic sites, which can also provide useful information on the chemical properties of molecular systems. FIREBALL-obtained condensed-to-atom Fukui functions of 10 atomic sites are shown in Figure 3.2, along with the corresponding HOMO-LUMO densities, which are listed in Table 3.1. 


\begin{tabular}{|c|c|c|c|}
\hline Site & $f^{-}$ & $f^{+}$ & $f^{+}-f^{-}$ \\
\hline 1 & 0.003061 & 0.006211 & 0.003150 \\
\hline 2 & 0.019307 & 0.007409 & -0.011898 \\
\hline 3 & 0.026334 & 0.009064 & -0.017271 \\
\hline 4 & 0.003282 & 0.023272 & 0.019990 \\
\hline 5 & 0.003315 & 0.023352 & 0.020036 \\
\hline 6 & 0.001466 & 0.015472 & 0.014006 \\
\hline 7 & 0.025601 & 0.018947 & -0.006654 \\
\hline 8 & 0.046545 & 0.013687 & -0.032858 \\
\hline 9 & 0.002040 & 0.010684 & 0.008644 \\
\hline 10 & 0.001573 & 0.013346 & 0.011773 \\
\hline 11 & 0.001605 & 0.013454 & 0.011848 \\
\hline
\end{tabular}

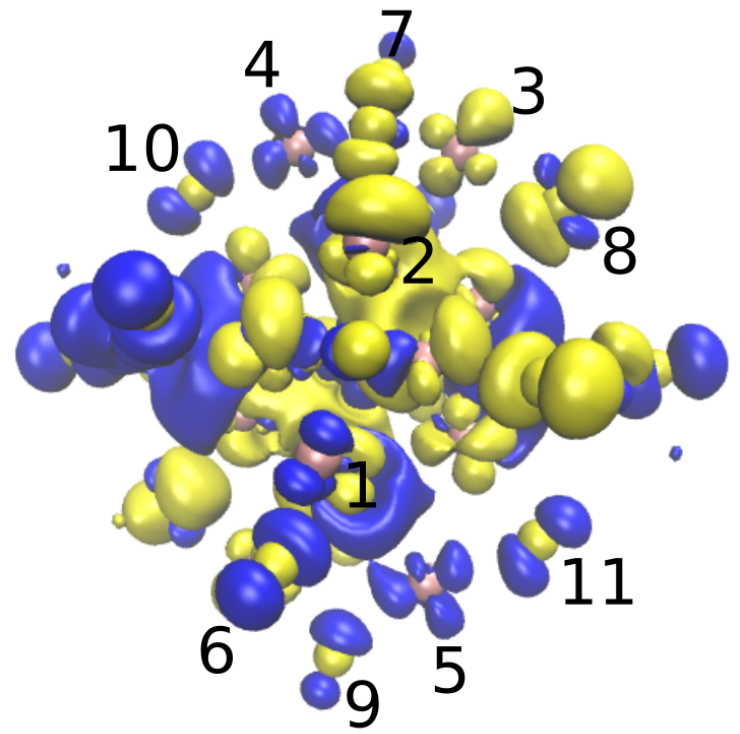

Figure 3.2: LUMO-HOMO electron densities of $\mathrm{Au}_{25}\left(\mathrm{SCH}_{3}\right)_{18}$

Table 3.1: Condensed to atom Fukui func-

tion values (based on Equation 3.4) corre-

sponding to 10 atomic sites

\subsection{Results and discussion}

First three subsections of this section discuss structural and electronic properties of $\mathrm{Au}_{25}(\mathrm{SR})_{18}, \mathrm{AgAu}_{24}(\mathrm{SR})_{18}, \mathrm{Ag}_{3} \mathrm{Au}_{25}(\mathrm{SR})_{18}$ and 6-8 $\mathrm{Ag}$ alloyed $\mathrm{Au}_{25}(\mathrm{SR})_{18}$. In 
the last subsection, we present our results on catalytic properties of moderately and heavily doped $\mathrm{Au}_{25}$.

\subsection{1 $\mathrm{Au}_{25}(\mathrm{SR})_{18}$ nanocluster}

$\mathrm{Au}_{25}(\mathrm{SR})_{18}$ nanoclusters with both 0 and -1 charge states have been experimentally realized. However, it has been shown, both experimentally and theoretically, that the structure with -1 charge state is the most stable. Structurally, there are small differences between neutral and negatively charged structures, which have been highlighted in reference 28. For our studies, we also used the negatively charged $\mathrm{Au}_{25}(\mathrm{SR})_{18}$ to facilitate comparison of our results with prior experimental and theoretical ones.

As the 13 atom core of $\mathrm{Au}_{25}(\mathrm{SR})_{18}$ cluster is an icosahedron, it contains three mutually perpendicular symmetry axes (see Figure 3.3 (a)). The 12 atoms in the outer shell are arranged as two pairs along each of these axes (see Figure 3.3 (b) ). Therefore the complete cluster possesses $C_{2}$ and $\sigma_{v}$ symmetry along the symmetry axes and inversion symmetry with respect to the center atom. Remarkably, inversion symmetry is observed to preserve even with the ligands. 

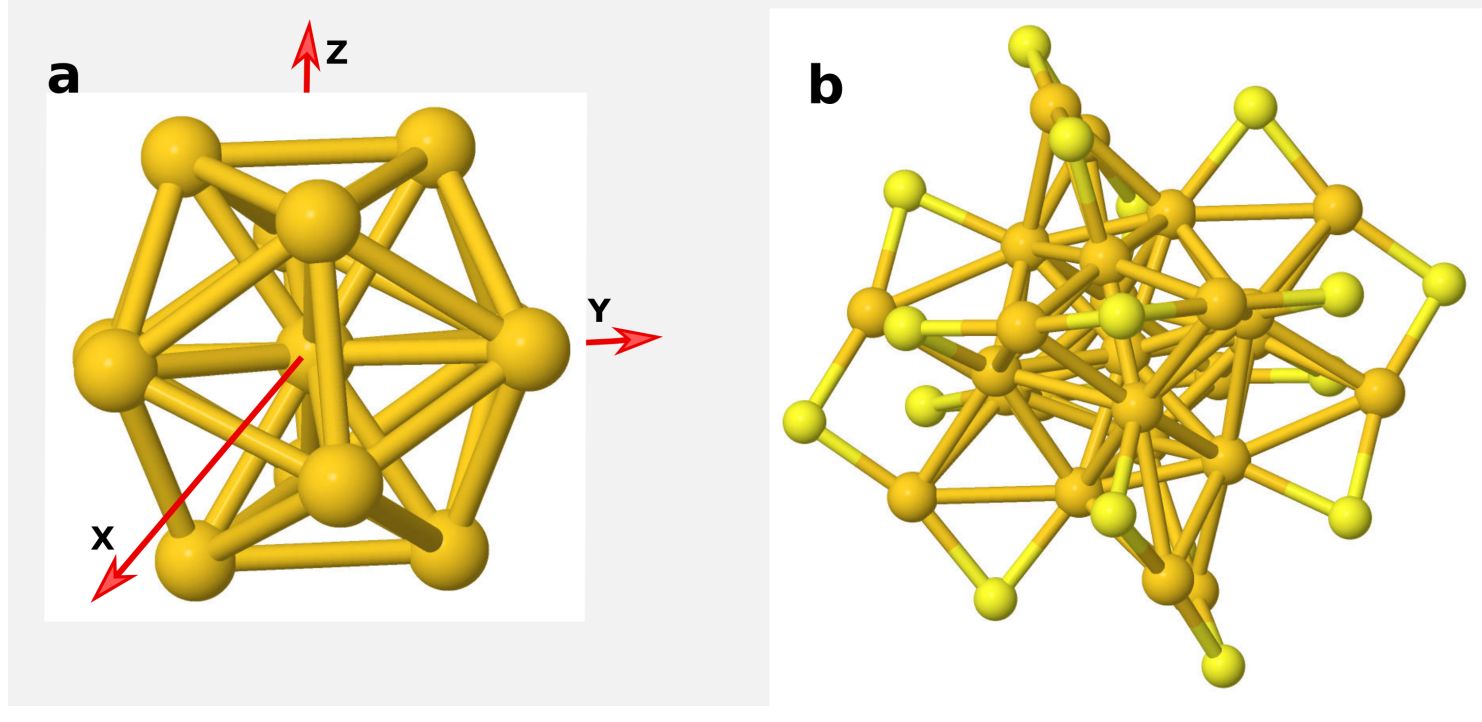

Figure 3.3: Symmetry axis of $\mathrm{Au}_{25}(\mathrm{SR})_{18}$

Bond lengths measured experimentally and calculated with FIREBALL are given in Table 3.4. Calculated Au-Au bond lengths show excellent agreement with the experimental results. Other Au-S bonds are slightly overestimated by Fireball, except for 4-5 bond. The differences between experimental and calculated bond lengths are sufficiently small. This small difference indicates that Fireball matrix elements are capable of capturing the correct structural trends in these nanoclusters.

Electronic density of states of $\mathrm{Au}_{25}(\mathrm{SR})_{18}$ is shown in Figure 3.6. HOMO and LUMO states of $\mathrm{Au}_{25}(\mathrm{SR})_{18}$ are composed mainly of Au-s,Au-p, Au-d and S-p states. Compared to $\mathrm{Au}$ and $\mathrm{S}$ states, $\mathrm{H}$ and $\mathrm{C}$ electron densities at HOMO and LUMO energies are negligible. The small $\mathrm{H}$ and $\mathrm{C}$ densities indicate that $-\mathrm{CH}_{3}$ fragments do not have a significant effect on the reactivity of $\mathrm{Au}_{25}$, in accordance with the frontier 


\begin{tabular}{|c|c|c|c|}
\hline Bond & Experimental & Firabll & Difference \\
\hline $1-2$ & 2.338 & 2.465 & -0.127 \\
\hline $2-3$ & 2.282 & 2.473 & -0.191 \\
\hline $3-4$ & 2.331 & 2.483 & -0.152 \\
\hline $4-5$ & 2.310 & 2.472 & +0.162 \\
\hline $5-6$ & 2.390 & 2.581 & -0.191 \\
\hline $1-7$ & 2.374 & 2.585 & -0.211 \\
\hline $2-7$ & 3.243 & 3.202 & 0.041 \\
\hline $4-6$ & 3.249 & 3.163 & 0.086 \\
\hline i-7 & 2.793 & 2.794 & -0.001 \\
\hline i-6 & 2.787 & 2.787 & 0.000 \\
\hline
\end{tabular}

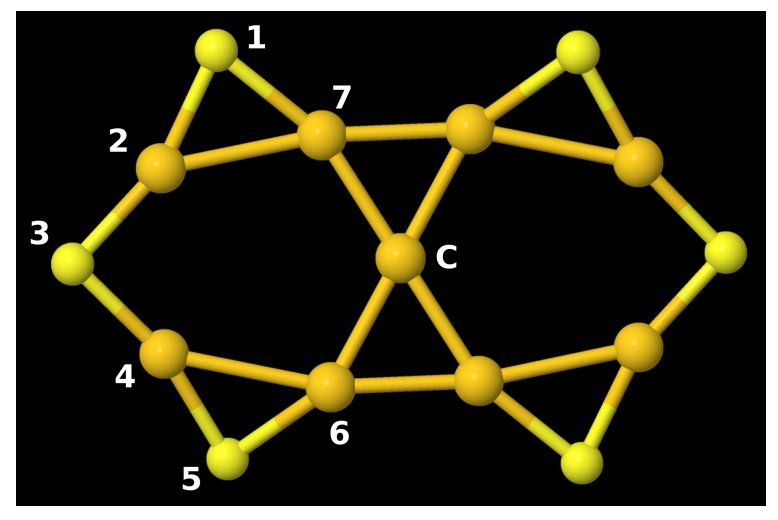

Figure 3.5: Au-Au and $\mathrm{Au}-\mathrm{S}$ bonds

Figure 3.4: Bond lengths in $\mathrm{Au}_{25}(\mathrm{SR})_{18}$ 
molecular orbital picture. The HOMO-LUMO gap calculated using electronic density of states (DOS) is about $1.35 \mathrm{eV}$ which underestimates the experimentally obtained value by about $0.4 \mathrm{eV}$.

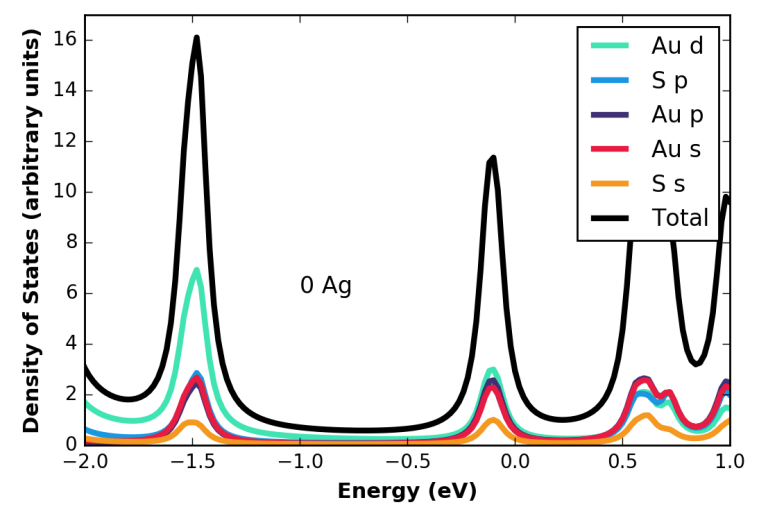

(a)

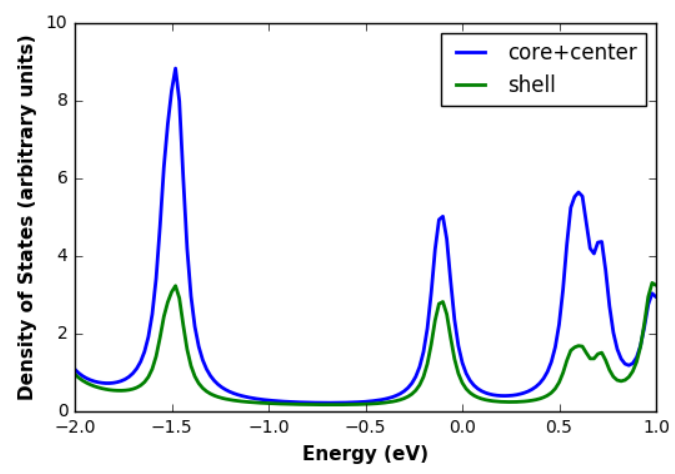

(b)

Figure 3.6: Electronic density of states (DOS) of (a) constituent atoms and (b) core and shell $\mathrm{Au}$ atoms of $\mathrm{Au}_{25}(\mathrm{SR})_{18}$

The electronic structure of thiolated nanoclusters are often described using the superatom theory ${ }^{159,160}$. This theory says that molecular orbitals of the nanoclusters have shapes similar to those of the atomic orbitals (s,p,d,...). Accordingly, these clusters are called superatoms: a collection of atoms having similar electron distribution as in isolated atoms. Superatomic orbitals are designated with uppercase letters $(\mathrm{S}, \mathrm{P}, \mathrm{D} \ldots)$ to distinguish them from their atomic counterparts. Figure 3.7 shows FIREBALL-obtained molecular orbitals corresponding to HOMO and LUMO states of $\mathrm{Au}_{25}\left(\mathrm{SCH}_{3}\right)_{18}$ which resemble atomic $\mathrm{p}$ and $\mathrm{d}$ states. This result is com- 
patible with the published theoretical data ${ }^{28}$. Therefore, in $\mathrm{Au}_{25}(\mathrm{SR})_{18}, \mathrm{HOMO}$ is a superatom P orbital and LUMO is a superatom D orbital. It should also be noted that HOMO is three-fold degenerate and LUMO is two-fold degenerate. In agreement with the DOS results, $\mathrm{CH}_{3}$ fragments do not contain much electron density in HOMO and LUMO states.

(a)

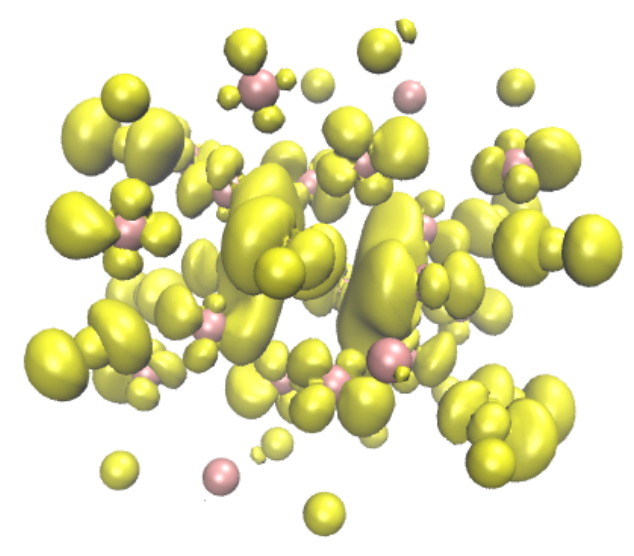

(b)

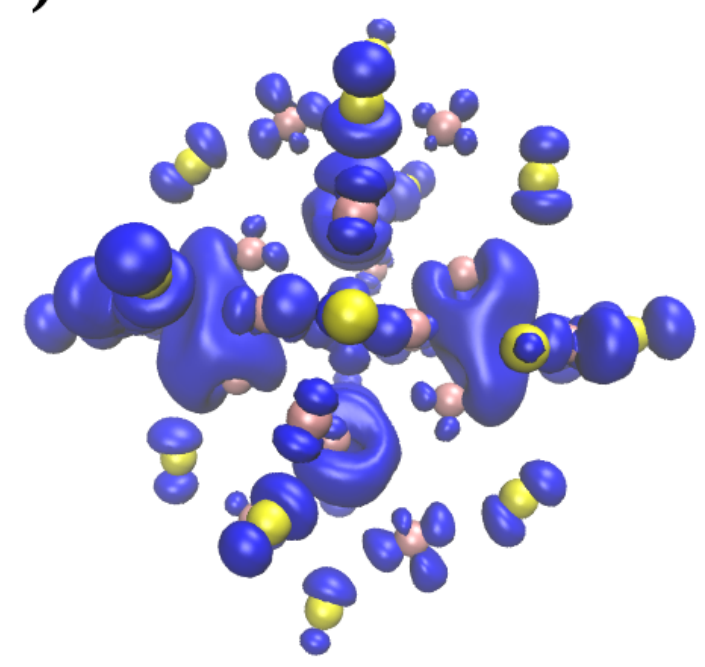

Figure 3.7: (a) HOMO and (b)LUMO electron densities of $\mathrm{Au}_{25}(\mathrm{SR})_{18}$

To ensure that our computations can produce structural trends that are consistent with the published experimental and theoretical results, we considered two cases where one and three $\mathrm{Au}$ atoms in $\mathrm{Au}_{25}(\mathrm{SR})_{18}$ are substituted with $\mathrm{Ag}$.

\subsection{2 $\quad \mathrm{AgAu}_{24}(\mathrm{SR})_{18}$ nanocluster}

All the studies carried out on single $\mathrm{Ag}$ atom substitution in $\mathrm{Au}_{25}(\mathrm{SR})_{18}$ have shown that the most favorable $\mathrm{Ag}$ locations are the surface sites of $\mathrm{Au}_{13}$ and $\mathrm{Ag}$ in the 
center results in one of the least favorable configurations. ${ }^{115,131}$ Figure 3.8 (a) shows our results on the preferable Ag dopant sites where color coded vertical lines indicate the location of $\mathrm{Ag}$ atoms in each of the 1-Ag alloyed $\mathrm{Au}_{25}\left(\mathrm{SCH}_{3}\right)_{18}$. In all the 12 lowest energy isomers, the $\mathrm{Ag}$ atom is in the surface of the $\mathrm{Au}_{13}$ icosahedron. This is strong evidence to conclude that these are the most preferable Ag alloying sites. The structure with a $\mathrm{Ag}$ atom in the center is among three least favorable isomers. According to FIREBALL, alloying the surface of $\mathrm{Au}_{13}$ and one of the staple sites has a minimum energy barrier of about $0.36 \mathrm{eV}$.

It is not uncommon to find studies done with - $\mathrm{SH}$ as the ligand, as it requires less computational resources than the calculations done with bigger ligands. However, as shown in Figure 3.8 (b), usage of -SH can have an effect on the structural trends. 


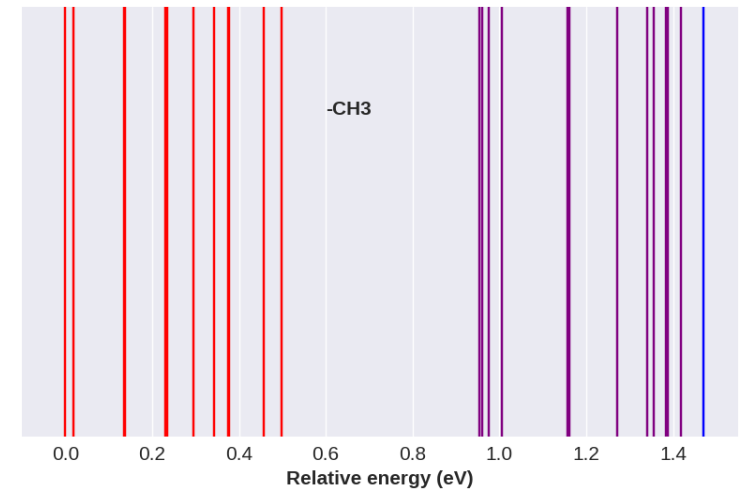

(a)

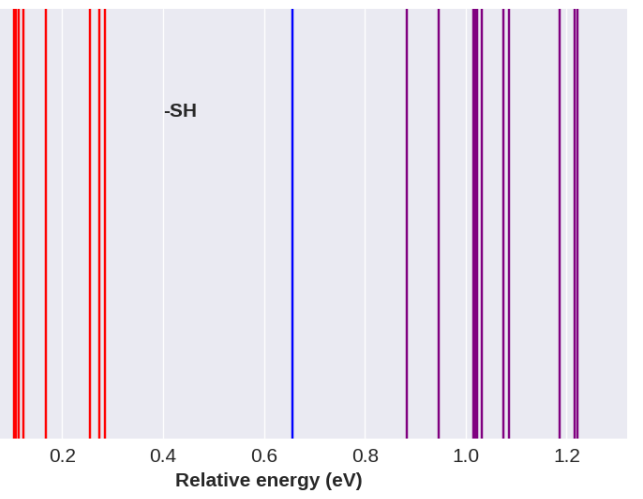

(b)

Figure 3.8: Structural energy of 1-Ag doped $\mathrm{Au}_{25}(\mathrm{SR})_{18}$ when (a) $\mathrm{CH}_{3}$ and (b)SH were used as the ligands. Different colors indicate the location of the Ag atom; red:surface of the $\mathrm{Au}_{13}$, purple:shell, blue:center

\subsection{3 $\mathrm{Ag}_{3} \mathrm{Au}_{22}(\mathrm{SR})_{18}$ nanocluster}

The first detailed study on three $\mathrm{Ag}$ atoms alloyed in $\mathrm{Au}_{25}$ was done by Kaufmann et al. ${ }^{137}$ According to them, energy decreases as the Ag configuration changes in this order: shell-shell-center, chell-core-center, shell-shell-shell, core-core-center, shellshell-core, shell-core-core, and, finally, core-core-core.

In the attempt to replicate these results, we considered all the possible 2300 3-Ag alloyed confirmations, including the symmetry equivalent and inequivalent doping sites. The corresponding results obtained with FIREBALL are shown in Figure 3.9 which agree with those of Kaufmanns' on the highest and and lowest energy con- 
figurations. The differences in the trends can be due to two main reasons: Kafumann et al. have considered only the symmetry non-equivalent isomers, while we considered all the possible 2300 isomers and the ligand used in their calculation is - $\mathrm{SH}$, whereas we modeled our isomers with $-\mathrm{SCH}_{3}$.

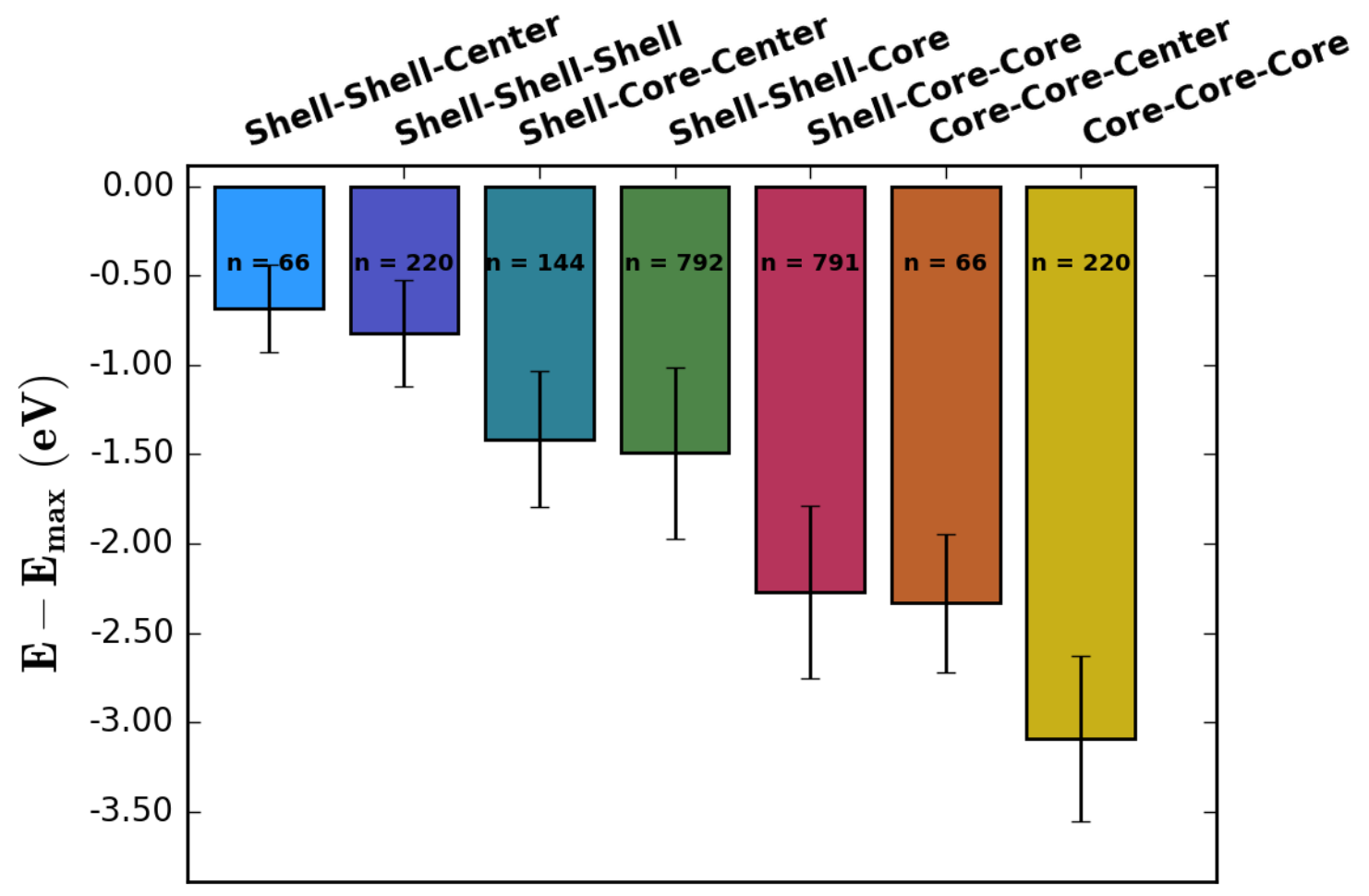

Figure 3.9: The effect of the location of $\mathrm{Ag}$ atoms on the total energy of $\mathrm{Au}_{22} \mathrm{Ag}_{3}\left(\mathrm{SCH}_{3}\right)_{18}$

An important observation in Figure 3.9 is that there is a significant reduction in structural energy for every $\mathrm{Ag}$ atom added to the $\mathrm{Au}_{13}$ surface of $\mathrm{Au}_{25}\left(\mathrm{SCH}_{3}\right)_{18}$. For example, the average energy difference between shell-shell-shell and shell-corecenter configurations is $0.59 \mathrm{eV}$. Moving one $\mathrm{Ag}$ to the $\mathrm{Au}_{13}$ surface reduces the 
energy further by $0.78 \mathrm{eV}$. Having three $\mathrm{Ag}$ atoms in the $\mathrm{Au}_{13}$ surface is energetically favorable by about $0.76 \mathrm{eV}$ compared to having two $\mathrm{Ag}$ atoms in the $\mathrm{Au}_{13}$ surface. Thus in terms of energy, the isomers can be categorized into four groups with, $0,1,2$, and $3 \mathrm{Ag}$ atoms in the $\mathrm{Au}_{13}$ surface.

\subsection{4 $\operatorname{Ag}_{x} \mathrm{Au}_{25-x}(\mathrm{SR})_{18}$ nanoclusters $(\mathrm{x} \geq 3)$}

At the time we started this work, the maximum number of $\mathrm{Ag}$ atoms that can be doped in $\mathrm{Au}_{25}(\mathrm{SR})_{18}$ was 7 . The composition of $\mathrm{Au}_{25-x} \mathrm{Ag}_{x}(\mathrm{SR})_{18}$ nanoclusters (from low silver content to moderate then to high silver content) was determined by matrixassisted laser desorption ionization mass spectroscopy (MALDI-MS), as shown in Figure 3.10. Each peak corresponds to a specific number of silver atoms doped into $\mathrm{Au}_{25}(\mathrm{SR})_{18}$, as labeled on the top of the Figure 3.10; for example, $\mathrm{x}=1$ indicates a single silver atom incorporated into the $\mathrm{Au}_{25}(\mathrm{SR})_{18}$ framework and results in the $\mathrm{AgAu}_{24}(\mathrm{SR})_{18}$ nanoalloy. The intensity of the peak in MALDI-MS corresponds to the relative abundance of each species, which depends on the reaction time. The longer the reaction time the larger is the number of $\mathrm{Ag}$ atoms corresponding to the dominant peak in the mass spectrum. ${ }^{38}$ 


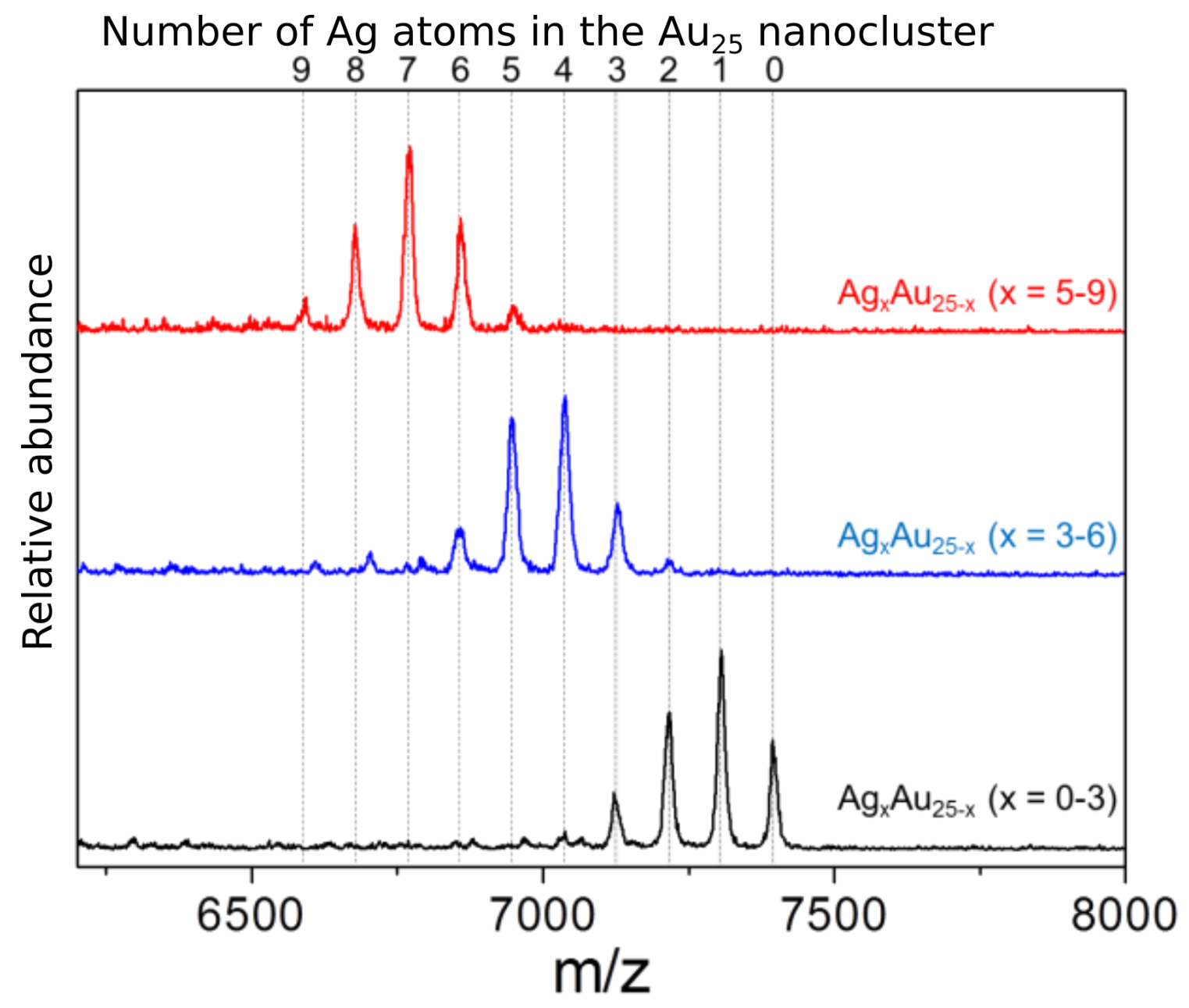

Figure 3.10: MALDI-MS of $\operatorname{Au}_{25-x} \operatorname{Ag}_{x}(\mathrm{SR})_{18}$ nanoalloys

However, the current synthesis procedures result in a mixture of alloyed species of $\mathrm{Au}_{25-x} \mathrm{Ag}_{x}$, with $x$ having a range of values as depicted in Figure 3.10. To obtain a clear picture of the alloyed structures and dopant impacts, our simulations focused on studying the individual alloying levels $(\mathrm{x}=6,7$ and 8$)$ of $\mathrm{Au}_{25-x} \mathrm{Ag}_{x}\left(\mathrm{SCH}_{3}\right)_{18}$ clusters. We used 100 structures with Ag atoms substituted at random Au sites for each alloying level in high-throughput calculations, with an exhaustive search for 
energetically favorable nanoparticles. We optimized all these structures until the root-mean-square of the force on the atoms fell below $0.05 \mathrm{eV} / \AA$.

Figure 3.11 shows the lowest energy isomers for each alloying level considered in this work. The Ag locations of the next four lowest energy isomers are given in Figure A.1 of Appendix A. We saw that the inversion symmetry of $\mathrm{Au}_{25}(\mathrm{SR})_{18}$ is preserved in the lowest energy 6-Ag alloyed structure. However, this is not the case in the next four lowest energy isomers. For the case of 7 silver atoms, we cannot expect to find a structure with $C_{i}$ symmetry unless its center is substituted with Ag. This is because for inversion symmetry to exist with an odd number of dopants, the center atom should be Ag, but we find that in the lowest energy structures, Ag atoms prefer the surface of the $\mathrm{Au}_{13}$ and avoid the center. Our lowest energy 8- $\mathrm{Ag}$ isomer also did not exhibit any symmetries of the unalloyed $\mathrm{Au}_{25}(\mathrm{SR})_{18}$ cluster.

(a)

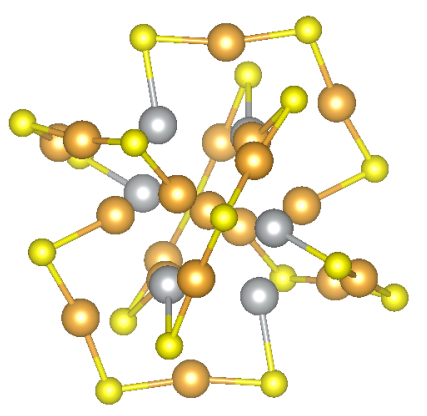

(b)

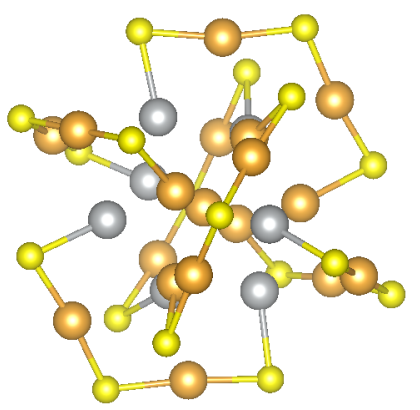

(c)

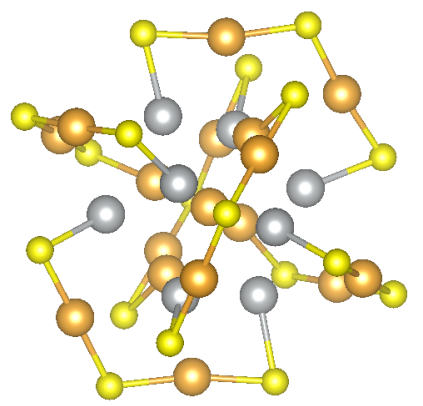

Figure 3.11: Lowest energy isomers of (a)6-, (b)7- and (c)8-Ag alloyed $\mathrm{Au}_{25}(\mathrm{SR})_{18}$

Figure 3.12 presents our results on the Ag locations in the three structural units (center, $\mathrm{Au}_{13}$ surface and outer shell) of $\mathrm{Au}_{25}$. We can deduce that the ener- 
getically favorable confirmations are the ones with a majority of the Ag atoms in the surface level of the $\mathrm{Au}_{13}$ icosahedron of the nanocluster. 


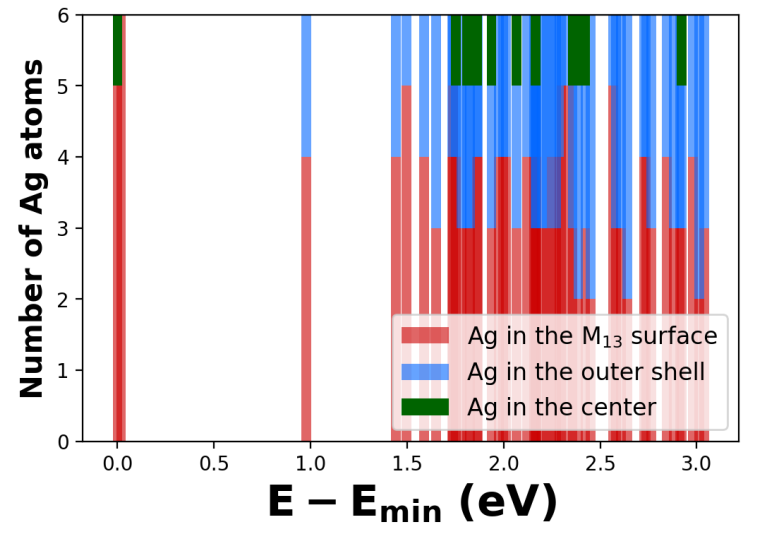

(a) $6-\mathrm{Ag}$

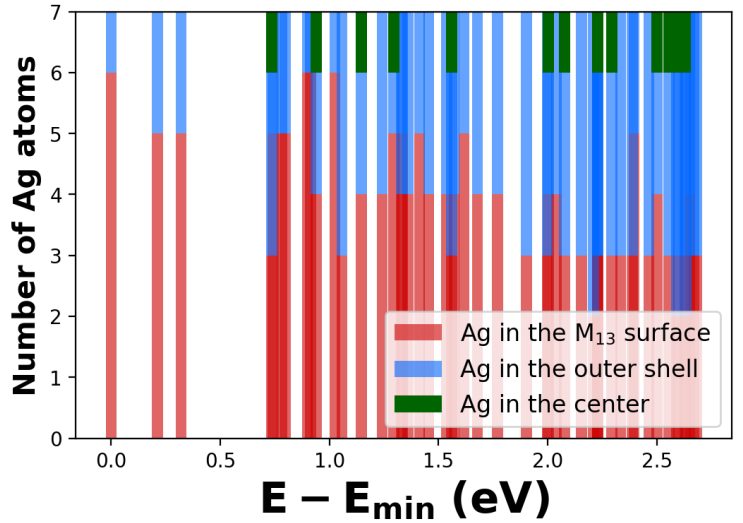

(b) $7-\mathrm{Ag}$

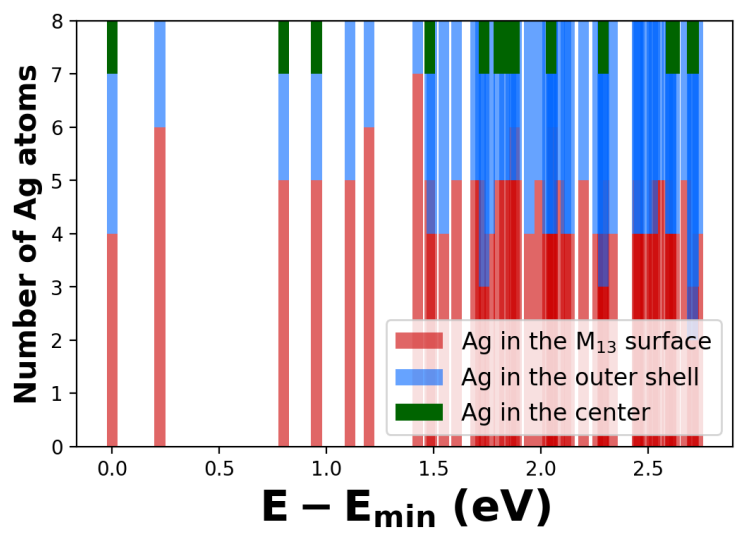

(c) $8-\mathrm{Ag}$

Figure 3.12: Location of Ag atoms in (a) 6-, (b) 7- and (c) 8-Ag alloyed nanoclusters obtained with random Ag substitution (only 50 lowest energy structures are shown for clarity). Red color bars correspond to the number of $\mathrm{Ag}$ atoms in the 13 atoms Metal surface $\left(\mathrm{M}_{13}\right)$. Blue color bars correspond to the number of $\mathrm{Ag}$ atoms in the outer shell and green indicates a Ag occupancy in the center. 
To investigate further on the preferred Ag locations, we created another batch containing 50 structures for each alloying level. But this time we confined Ag atoms only to the $\mathrm{Au}_{13}$ icosahedron and the $\mathrm{Ag}$ sites in the $\mathrm{Au}_{13}$ icosahedron have been selected randomly. In Figure 3.13, we show the Ag locations of the 10 lowest energy isomers corresponding to this new batch (with $\mathrm{Ag}$ atoms biased to the $\mathrm{Au}_{13}$ core) together with those corresponding to the calculations done with randomly chosen $\mathrm{Ag}$ sites. We can see that the isomers with all the Ag atoms in the surface of the $\mathrm{Au}_{13}$ core are indeed the energetically favorable ones. This result agrees with the experimental findings of Kumara et al. who probed the structure of $\mathrm{Au}_{25}$ alloyed with roughly six Ag atoms using X-ray crystallography. Their data showed that the probability to find a $\mathrm{Ag}$ atom in the center or in the outer shell is zero. 


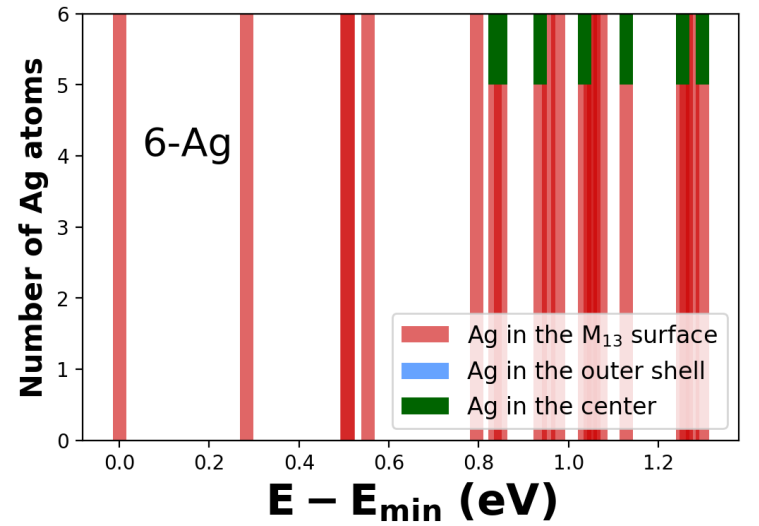

(a) $6-\mathrm{Ag}$

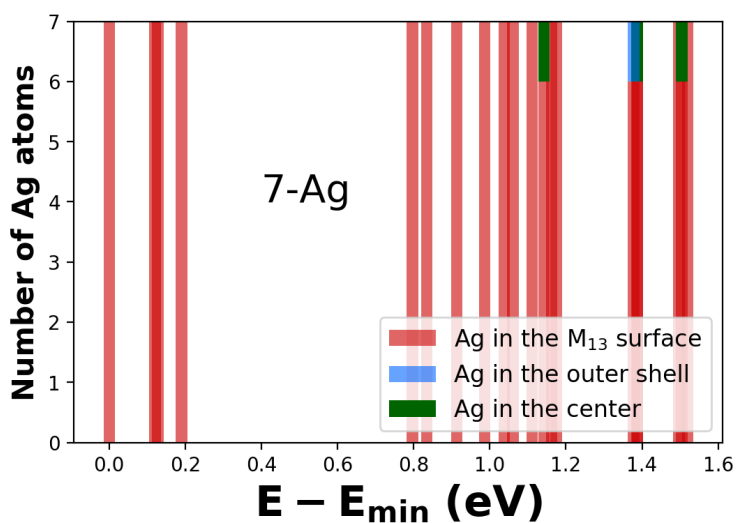

(b) $7-\mathrm{Ag}$

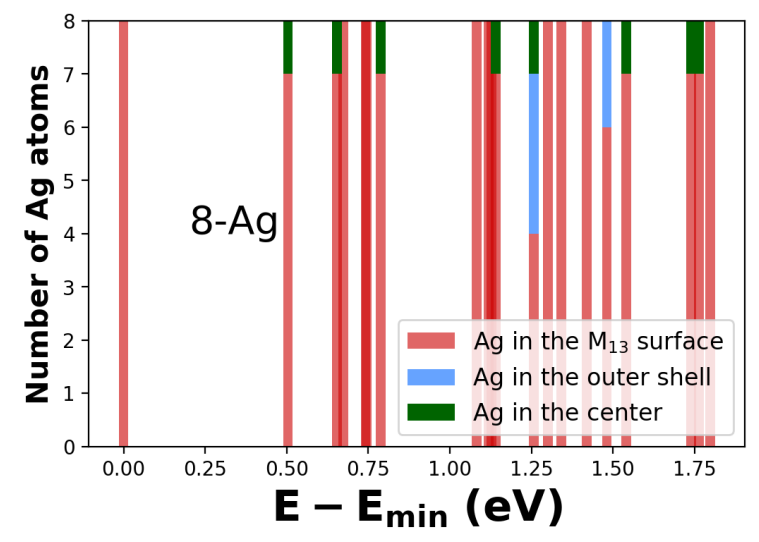

(c) $8-\mathrm{Ag}$

Figure 3.13: Location of the Ag atoms of the 10 lowest energy isomers corresponding to calculations done with $\mathrm{Ag}$ confined to the $\mathrm{Au}_{25}$ core and with randomly chosen $\mathrm{Ag}$ sites.

Guidez et al. reported that with $X \alpha$ level of theory, and using three isomers with two $\mathrm{Ag}$ atoms in the surface of $\mathrm{Au}_{13}$ icosahedron, structural energy reduces as 
the distance between two Ag atoms increases. ${ }^{115}$ To check whether there are any correlations between the clustering of the Ag atoms and the structural stability, we defined a clustering factor, which is simply the mean of the distances between the Ag atoms and their centroid position. Thus, the lower the clustering factor, the closer the Ag atoms are to each other.

$$
\text { Clustering factor }=\frac{1}{N} \sum_{i}\left|\vec{r}_{A g, i}-\vec{r}_{c}\right|
$$

Here, $i$ runs over all the $N$ dopant $\mathrm{Ag}$ atoms and $r_{c}$ is the mean position(centroid) of these Ag atoms.

Our results on clustering factors for 2,6,7,and 8-Ag alloyed clusters are shown in Figure 3.14. We see that the clustering factors for $\mathrm{x}=2$ are grouped mainly around four values. The structures with the highest clustering factors are the ones with both Ag atoms in the staples. Agreeing with the result of Guidez et al., Ag atoms in the lowest energy isomer are located at either side of the center atom, maximizing the distance between them. However, there are 5 other structures with the same Ag configuration with energies as high as the ones with lower clustering factors. Therefore, the hypothesis of Guidez et al. is not valid when all the isomers are considered. Also for 6,7, and 8-Ag alloyed structures, higher clustering factors do not correspond to lower energies, even when only the few lowest energy structures are considered. 


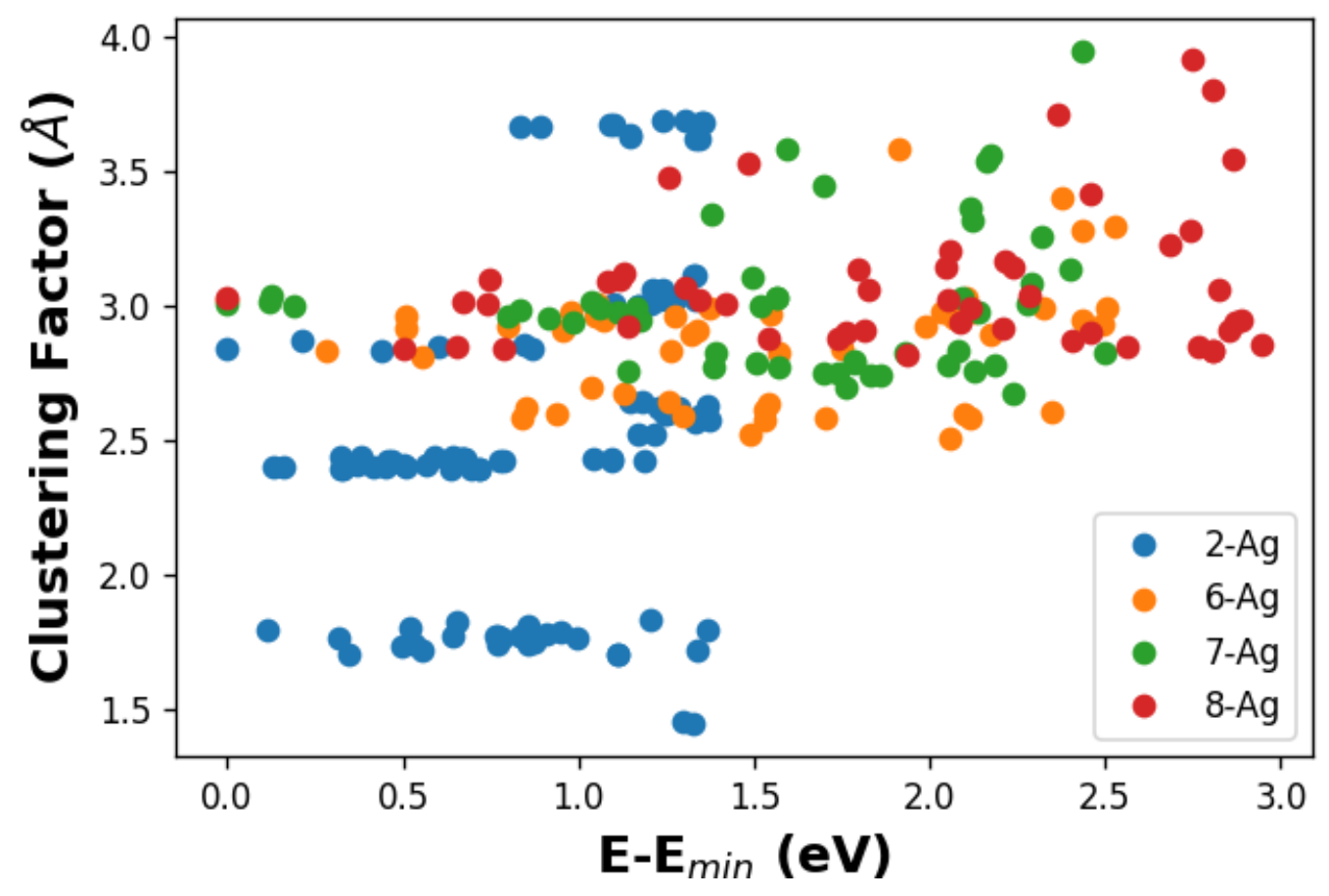

Figure 3.14: Clustering factors in 2,6,7,8 $\mathrm{Ag}$ alloyed $\mathrm{Au}_{25}(\mathrm{SR})_{18}$

Electronic properties

Previously published experimental work on the $\mathrm{Ag}$ alloying in $\mathrm{Au}_{25}(\mathrm{SR})_{18}$ shows that the photon absorption energy increases with the increasing number of Ag atoms in the cluster. ${ }^{33}$ This experimental data also shows a significant broadening in the absorption peaks at the vicinity of the main absorption peak of the Ag alloyed species. Our density of states (DOS) results, shown in Figure 3.15, are in agreement with this observation. In Figure 3.15 (e), the weighted average DOS is presented, of which the weights reflect the relative abundance of each Ag alloyed species, which is based on the height of the peaks in the mass spectrum. In the un-alloyed structure, there are 
two sharp peaks in the DOS, corresponding to the HOMO and LUMO levels (Figure 3.15 (a)) and a region without any states immediately after the LUMO level. The absence of states can limit electron transitions at these energies, resulting in very low absorption intensities. This feature is absent in the $\mathrm{Ag}$ alloyed structures. Instead of a single dominant peak, the LUMO level is composed of a broadened region of many electronic states in the alloyed nanoclusters. The availability of more LUMO states enables higher energy HOMO-LUMO transitions than the ones allowed in the un-alloyed structure. Due to higher HOMO-LUMO transitions, the average photon absorption energy can increase, creating a peak at a higher energy value in the photon absorption spectrum of Ag alloyed structures. A closer look at the DOS reveals that this broadening is mediated mainly by the newly formed silver S states (Figure 3.16). As the number of $\mathrm{Ag}$ atoms increases, the number of these $\mathrm{S}$ states at the LUMO level also increases, thereby amplifying the broadening. The weighted average DOS (Figure 3.15 (e)) further confirms the emergence of new states between 0 and 0.5 $\mathrm{eV}$ as an effect coming from the LUMO electronic states in 6-, 7-, and 8-Ag alloyed nanoclusters. The HOMO-LUMO energy gap for the $\mathrm{Au}_{25-x} \mathrm{Ag}_{x}\left(\mathrm{SCH}_{3}\right)_{18}$ is about $1.5 \mathrm{eV}$, which is comparable to the experimental absorption peak at $1.8 \mathrm{eV}$ in the UV-vis spectrum reported by Zhu et al. ${ }^{28}$ 


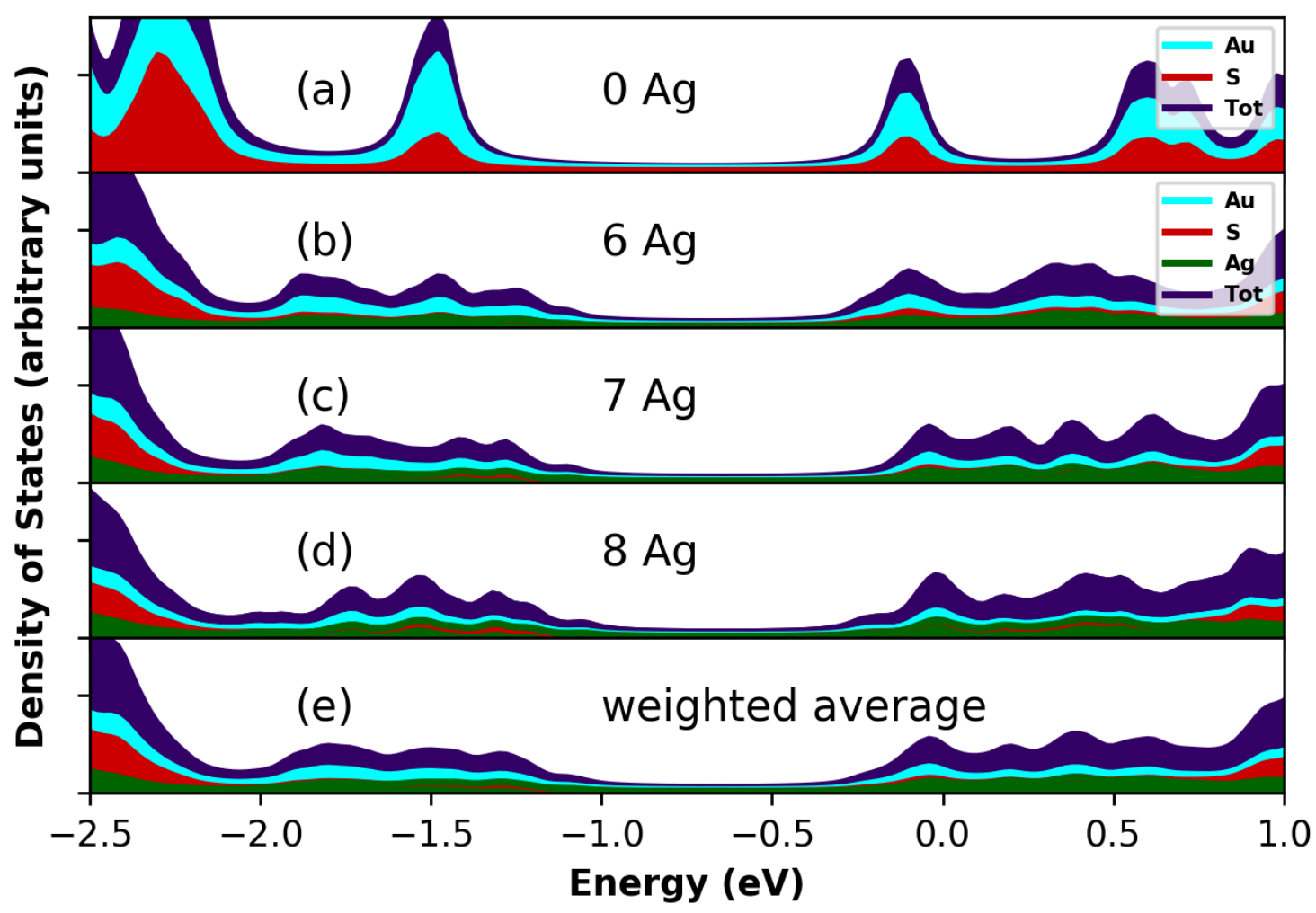

Figure 3.15: Density of states (DOS) and partial density of states (PDOS) for (a) 0-, (b) 6-,(c) 7- and (e) 8-Ag alloyed nanoclusters in comparison with the (e) weighted average DOS and PDOS over 6,7, and 8-Ag cases. (Weights are based on the height of the peaks in the MALDI-MS (shown in Figure 3.10).) 
for $\mathrm{i}$ in $[1,3,5,7,11,12,13]$ :

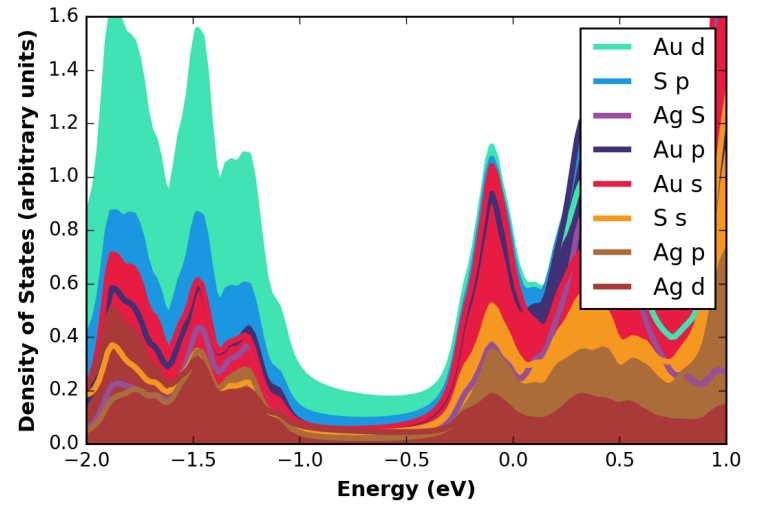

(a)

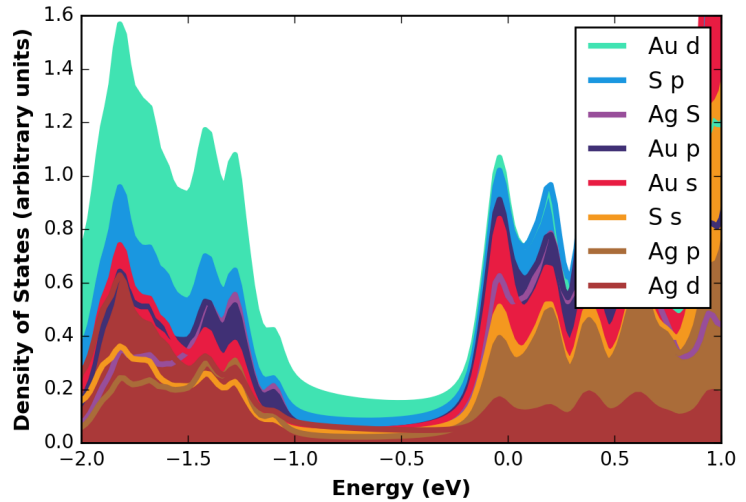

(b)

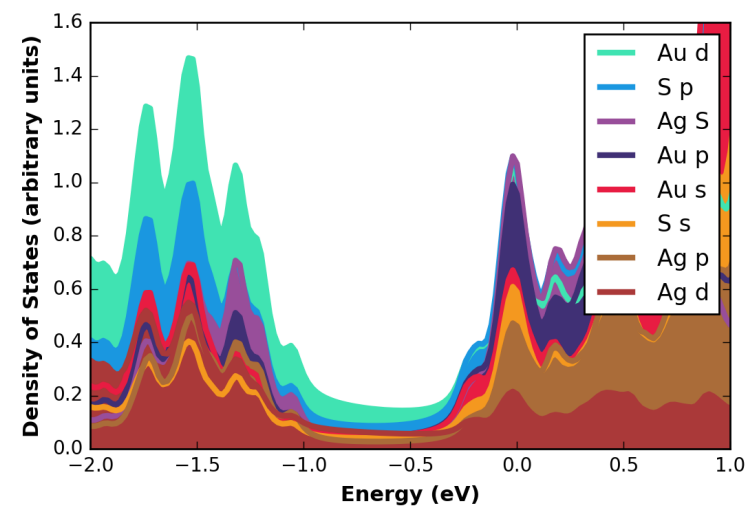

(c)

Figure 3.16: Orbital DOS of 6-,7-,and 8-Ag alloyed $\mathrm{Au}_{25}\left(\mathrm{SCH}_{3}\right)_{18}$

Figure 3.17 shows the HOMO and LUMO densities of the lowest energy alloyed isomers. Note that the these images are oriented to highlight the superatomic orbitals and the location of the Ag atoms. Thus, HOMO and LUMO density images for a given alloying level were not plotted with the same orientation. The superatomic 
$\mathrm{P}$ and $\mathrm{D}$ nature of the HOMO and LUMO states observed for the unalloyed case is still preserved to some extent in the alloyed isomers. The lowest energy 6-Ag alloyed isomer has its $\mathrm{Ag}$ atoms in symmetric positions and the majority of the HOMO density is distributed at these Ag atoms. However, this is not visible in the next four lowest energy isomers which do not show any symmetry(given in the appendix). This is also the case with the frontier orbital densities of the 7 and $8-\mathrm{Ag}$ alloyed lowest energy isomers. 
(a) 6-Ag HOMO

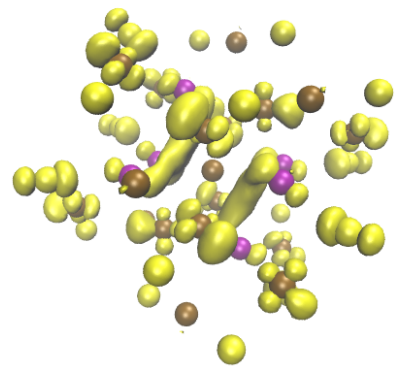

(d) 6-Ag LUMO

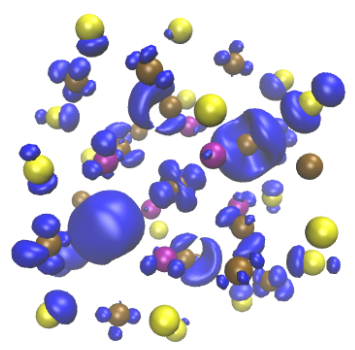

(b) 7-Ag HOMO

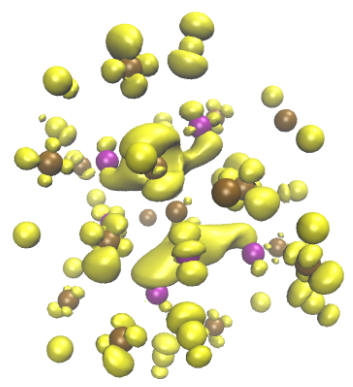

(e) 7-Ag LUMO

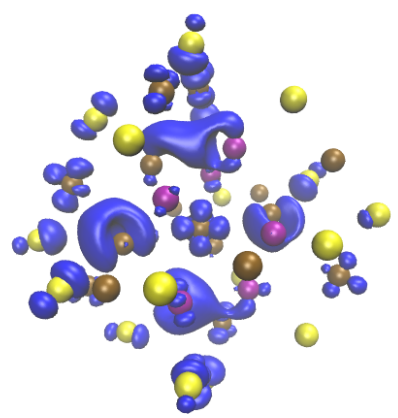

(c) 8-Ag HOMO

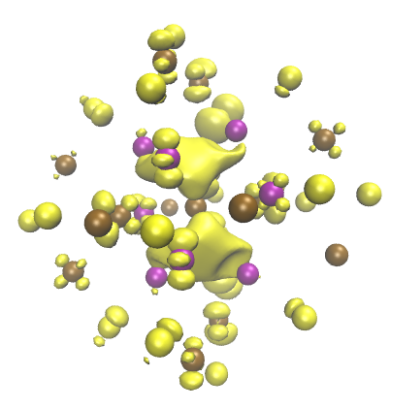

(f) 8-Ag LUMO

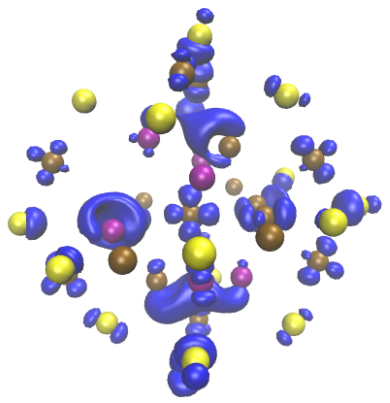

Figure 3.17: HOMO and LUMO electron densities of lowest energy isomers of $\mathrm{Au}_{25-x} \mathrm{Ag}_{x}\left(\mathrm{SCH}_{3}\right)_{18}$ for $\mathrm{x}=6,7,8$. Image orientation was decided such that the location of the Ag atoms and the superatom orbitals are visible. Brown: gold; purple: silver; yellow: sulfur. Isovalue $=0.006$ 


\subsubsection{Catalytic properties of moderately and heavily doped $\mathrm{Au}_{25}$}

To find how the catalytic activity of bimetallic $\mathrm{Au}_{25}(\mathrm{SR})_{18}$ changes with the amount of $\mathrm{Ag}$, our collaborators at the Carnegie Mellon University measured $\mathrm{CO} \mapsto \mathrm{CO}_{2}$ conversion rates in the presence of $\mathrm{Au}_{25}(\mathrm{SR})_{18}$ as the catalyst at different temperatures. Their results, shown in Figure 3.18, indicate that the conversion percentages for 4- and 7- Ag alloying levels are less than the conversion percentages for unalloyed $\mathrm{Au}_{25}(\mathrm{SR})_{18}$ for temperatures below $130{ }^{\circ} \mathrm{C}$. Before presenting our calculations, it is important to understand the details of the experimental procedure carried out to obtain the results shown in Figure 3.18.

Experimental methodologies

$\mathrm{Ag}_{x} \mathrm{Au}_{25}\left(\mathrm{SC}_{2} \mathrm{H}_{4} \mathrm{Ph}\right)_{18}(\mathrm{x}=0-9)$ nanoclusters were deposited onto $\mathrm{CeO}_{2}$ powders $(200$ mg) by wet deposition.44 After stirring for $24 \mathrm{~h}$, all the nanoclusters were adsorbed onto $\mathrm{CeO}_{2}$ support, as the supernatant became colorless. The as-prepared catalysts were dried at ambient temperature and no further treatment was performed unless otherwise noted. $100 \mathrm{mg}$ of $\mathrm{Ag}_{x} \mathrm{Au}_{25-x}\left(\mathrm{SC}_{2} \mathrm{H}_{4} \mathrm{Ph}\right)_{18}(\mathrm{x}=0-9) / \mathrm{CeO}_{2}$ was mixed with quartz wool and tested for $\mathrm{CO}$ oxidation in a fixed-bed, continuous flow reactor (8 mm i.d.) under ambient pressure.45 Before CO oxidation test, the as-prepared catalysts were pretreated at $150{ }^{\circ} \mathrm{C}$ in diluted $\mathrm{O}_{2}\left(\mathrm{O}_{2} / \mathrm{He}=10 / 90\right.$ volume $)$ for $1 \mathrm{~h}$ and then cooled down to room temperature. The reaction gas mixture of $3 \% \mathrm{CO}, 10 \%$ O2 and $87 \%$ He flowed through the reaction bed at a flow rate of $40 \mathrm{~mL} \mathrm{~min}{ }^{-1}$. The reaction products were analyzed by an online gas chromatograph (HP 6890) 
equipped with a carbon molecular sieve column and a thermal conductivity detector. Matrix-assisted laser desorption ionization (MALDI) mass spectrometry was performed with a PerSeptive-Biosystems Voyager DE super-STR time-of-flight (TOF) mass spectrometer. trans-2-[3-(4-tert-Butylphenyl)-2-methyl-2-propenyldidene] malononitrile (DCTB) was used as the matrix. Typically, $0.5 \mathrm{mg}$ of matrix and $0.005 \mathrm{mg}$ of $\mathrm{Ag}_{x} \mathrm{Au}_{25-x}\left(\mathrm{SC}_{2} \mathrm{H}_{4} \mathrm{Ph}\right)_{18}$ nanocluster (i.e., a 100[thin space (1/6-em)]:[thin space (1/6-em) $] 1$ mass ratio between DCTB and sample) were mixed in $50 \mu \mathrm{L}$ of $\mathrm{CH}_{2} \mathrm{Cl}_{2}$.

A $10 \mu \mathrm{L}$ portion of solution was applied to the steel plate and air-dried.

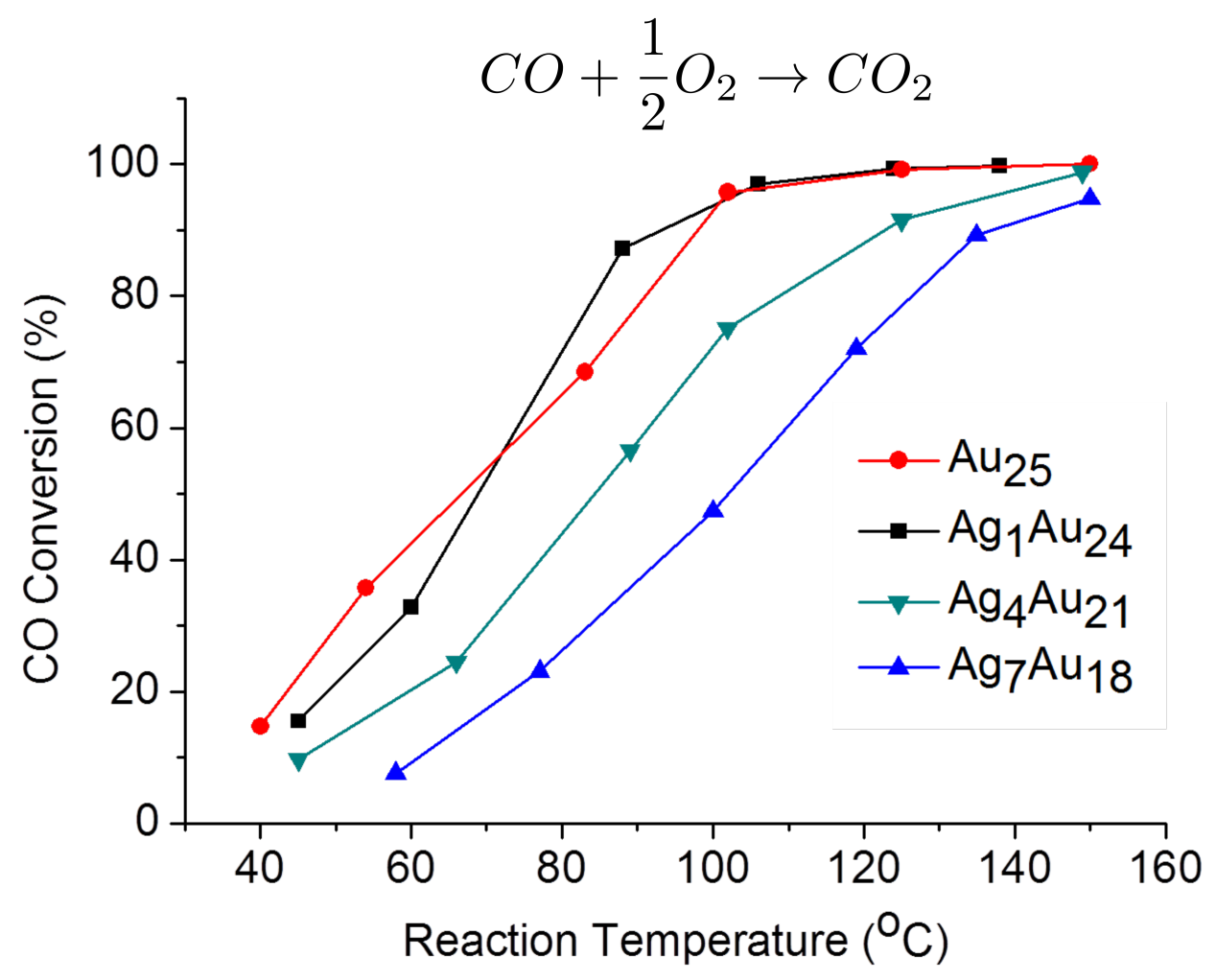

Figure 3.18: Temperature dependent CO conversion rates 
Fukui calculations

In order to figure out a theoretical reason for the reduction in catalytic activity, we employed condensed-to-atom Fukui functions. The Fukui values were obtained by finding the charge on each atom of ground state, one electron added and one electron removed systems. To show that FIREBALL can replicate the trends in Fukui values reoprted by other researchers, we calculated condensed-to-atom Fukui values of $\mathrm{Au}_{55}$ nanocluster. Using PBE as implemented in the OpenMX electronic structure code, Allison and Tong showed that electrophilic attack indices $\left(f^{-}\right)$of vertex sites are greater than edge site $f^{-}$values. ${ }^{161}$ Vertex sites are defined as Au atoms that make five nearest neighbor bonds where as atoms with seven nearest-neighbor bonds are identified as edge sites. Table 3.2 lists $f^{-}$values corresponding to five edge and vertex sites obtained by FIREBALL, which agree with the trend reported in the work of Allison and Tong. 


\begin{tabular}{|l|l|}
\hline Edge & Vertex \\
\hline \hline 0.0169 & 0.0359 \\
\hline 0.0167 & 0.0360 \\
\hline 0.0238 & 0.0372 \\
\hline 0.0168 & 0.0371 \\
\hline 0.0187 & 0.0360 \\
\hline
\end{tabular}

Table 3.2: FIREBALL-obtained $\mathrm{f}_{k}^{-}$values corresponding to five edge and vertex sites in $\mathrm{Au}_{55}$.

As the ligand plays an important role in the catalytic activity, we generated structures with the ligand used in the experimental CO oxidation, which is $\mathrm{CH}_{2} \mathrm{CH}_{2} \mathrm{Ph}$. It is important to remind that the nanoparticles which give rise to the $\mathrm{Ag}_{4} \mathrm{Au}_{21}$ and $\mathrm{Ag}_{7} \mathrm{Au}_{18}$ curves in Figure 3.18 are composed of clusters containing 3-6 and 5-9 Ag atoms respectively. Therefore, our condensed Fukui calculations should take account of the contributions from all these clusters. For 3-9 Ag alloyed cases, 100 structures were generated. In 50 of the them, dopant sites were chosen randomly from the $\mathrm{Au}_{13}$ core. This is because, according to previous calculations with $\mathrm{CH}_{3}$, these sites were found to be the favorite dopant sites. No such restriction was imposed on the selection of dopant sites in the other 50 structures.

The composition of these structures in terms of the number of Ag atoms at 
the center, the surface of $\mathrm{Au}_{13}$ icosahedron and the shell sites are shown in Figures A.2 and A.3 in Appendix A.

We selected 15 lowest energy isomers from each Ag alloyed sample and calculated the dual descriptor values corresponding to the outer shell sites of these isomers according to Equation 3.2. We take into account the coexistence of different Agalloyed species in the same experimental mixture by defining a weighted average dual descriptor value as given in Equation 3.5.

$$
\Delta f_{k}^{a v g}=\sum_{x} a_{x} \sum_{j}^{N \text { isomers }} \Delta f_{k, j} \times W_{j}
$$

In Equation 3.5, $k$ stands for a given atomic site and $x$ represent the number of Ag atoms. Boltzmann weight (Equation 3.3) for each isomer based on their relative energies.

$$
W_{i}=\frac{e^{-\left(E_{i}-E_{m i n}\right) /\left(k_{B} T \times N\right)}}{\sum e^{-\left(E_{i}-E_{m i n}\right) /\left(k_{B} T \times N\right)}}
$$

Here, $E_{i}$ is the total energy of an isomer, $k_{B}$ is the Boltzmann constant, $N$ is the number of atoms in each isomer. The largest changes in the CO conversion percentages in Figure 3.18 are observed in the reaction temperature range $85^{\circ}$ to $100^{\circ}$. Therefore in Equation 3.5, $T$ was chosen to be $373.15 \mathrm{~K}\left(100^{\circ}\right)$.

The weighted average dual descriptor values corresponding to each outer shell site of the alloyed isomers are shown in Figure 3.19. As previously mentioned, sites with positive $\Delta f$ values prefer to attract electrons and the ones with negative $\Delta f$ values prefer to donate electrons. In the unalloyed structure, majority of the outer 
shell sites prefer to donate electrons. However in both 3-6 and 5-9 Ag alloyed isomers, the tendency of the outer shell sites to donate electrons has reduced compared to that of the unalloyed structure. Now the question is, how the electron-donating capability of $\mathrm{Au}_{25}$ affects $\mathrm{CO}$ oxidation? A precise answer to this question cannot be provided as the exact mechanism of $\mathrm{CO}$ oxidation with $\mathrm{Au}_{25}$ is not known yet.

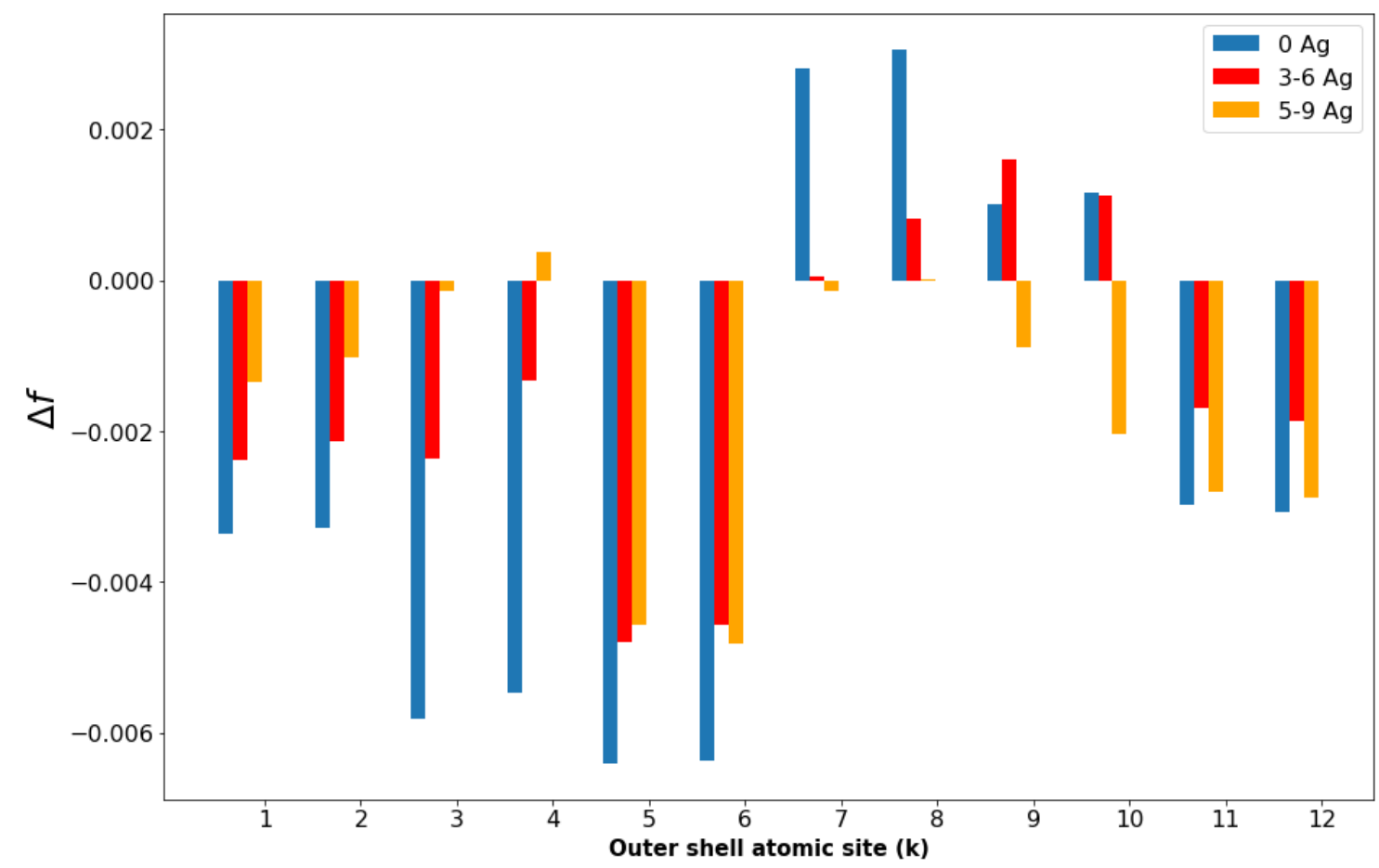

Figure 3.19: Weighted average dual descriptor values corresponding unalloyed, 3-6 and 5-9 $\mathrm{Ag}$ alloyed $\mathrm{Au}_{25}\left(\mathrm{SCH}_{2} \mathrm{CH}_{2} \mathrm{Ph}\right)_{18}$.

However, current experimental evidence by Nie et al. shows that $\mathrm{O}_{2}$ pretreatment dramatically increases $\mathrm{CO}$ oxidation. ${ }^{162}$ They further argue that $\mathrm{CO}$ oxidation takes place at the interface between $\mathrm{Au}_{25}$ nanocluster and the $\mathrm{CeO}_{2}$ substrate. $\mathrm{By}$ 
testing catalytic performance with different substrates $\left(\mathrm{TiO}_{2}, \mathrm{FeO}_{2}\right.$ and $\left.\mathrm{CeO}_{2}\right)$, they showed that the use of $\mathrm{CeO}_{2}$ maximizes the $\mathrm{CO}_{2}$ conversion. They believe that $\mathrm{O}_{2}$ pretreatment creates an abundance of active $\mathrm{O}_{2}$ species at the interracial region between $\mathrm{Au}_{25}$ and $\mathrm{CeO}_{2}$ (shown in Figure 3.20). Obviously, the presence of active $\mathrm{O}_{2}$ is vital for efficient $\mathrm{CO}$ to $\mathrm{CO}_{2}$ conversion. They also argue based on their previous results on conversion of $\mathrm{Au}_{25}^{-}$to $\mathrm{Au}_{25}^{0}$ by air oxidation, $\mathrm{Au}_{25}$ spontaneously donate electrons to $\mathrm{O}_{2} \cdot{ }^{163}$ And the acceptance of electrons by $\mathrm{O}_{2}$ forms active $\mathrm{O}_{2}$ species, like $\cdot \mathrm{O}_{2}$. Obviously, the reduction in the tendency to donate electrons in Ag alloyed $\mathrm{Au}_{25}$ as indicated by our calculations, adversely affects the formation of active oxygen. Therefore we believe that this reduction in the electron-donating capability of outer shell sites is highly likely to be a major contributing factor for the observed reduction in the catalytic activity of $\mathrm{Ag}$ alloyed $\mathrm{Au}_{25}$.

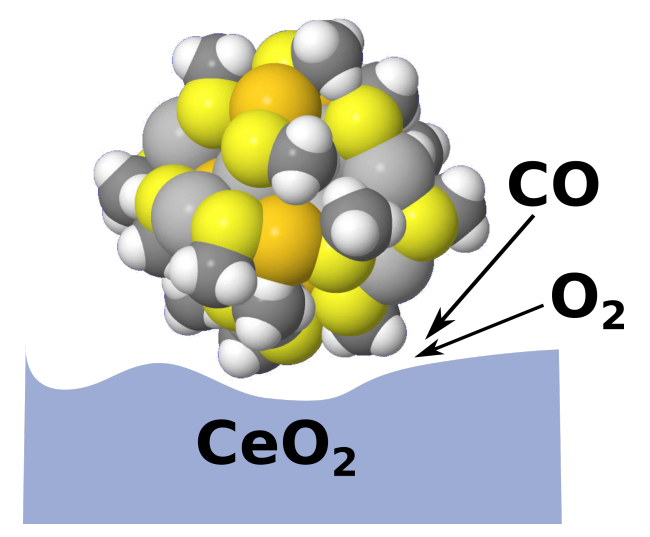

Figure 3.20: Current belief is that $\mathrm{O}_{2}$ species at the interracial region between $\mathrm{Au}_{25}$ and $\mathrm{CeO}_{2}$ are vital for $\mathrm{CO}$ oxidation. 
Heavily doped $\mathrm{Au}_{25-x} \mathrm{Ag}_{x}(\mathrm{SR})_{18}(\mathrm{x}=19-23)$

To study the catalytic activity of heavily doped bimetallic $\mathrm{Au}_{25}(\mathrm{SR})_{18}$ structures, S-c$\mathrm{C}_{6} \mathrm{H}_{11}$ ligand was used in accordance with the experimental requirement that heavily doped isomers can only be synthesized using this ligand. The ESI mass spectra for these heavily doped $\mathrm{Au}_{25}$ indicates that the number of $\mathrm{Ag}$ atoms in these clusters range from 19 to $23 .{ }^{164}$ Accordingly, for each alloying level, we created 100 isomers having randomly chosen Ag sites. Figure A.4 in Appendix A shows that the number of $\mathrm{Ag}$ atoms in different structural units in $\mathrm{Au}_{25}\left(\mathrm{SC}_{6} \mathrm{H}_{11}\right)_{18}$.

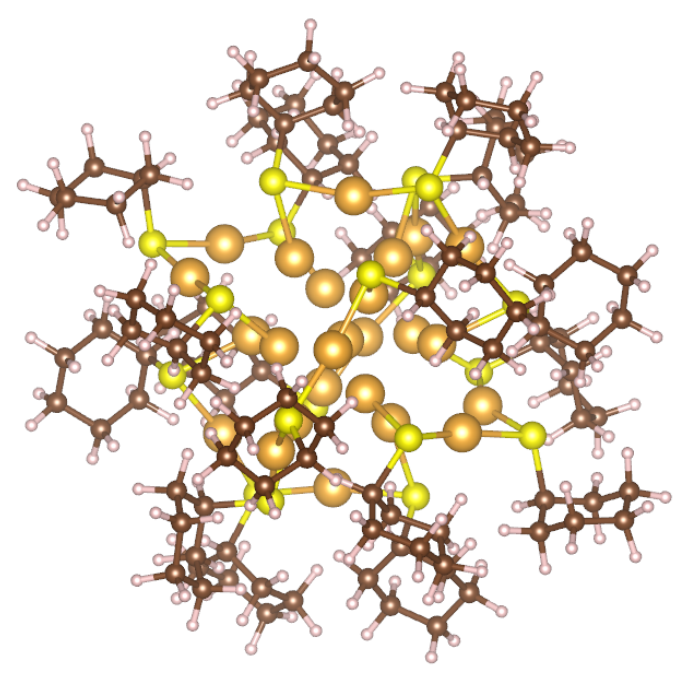

(a)

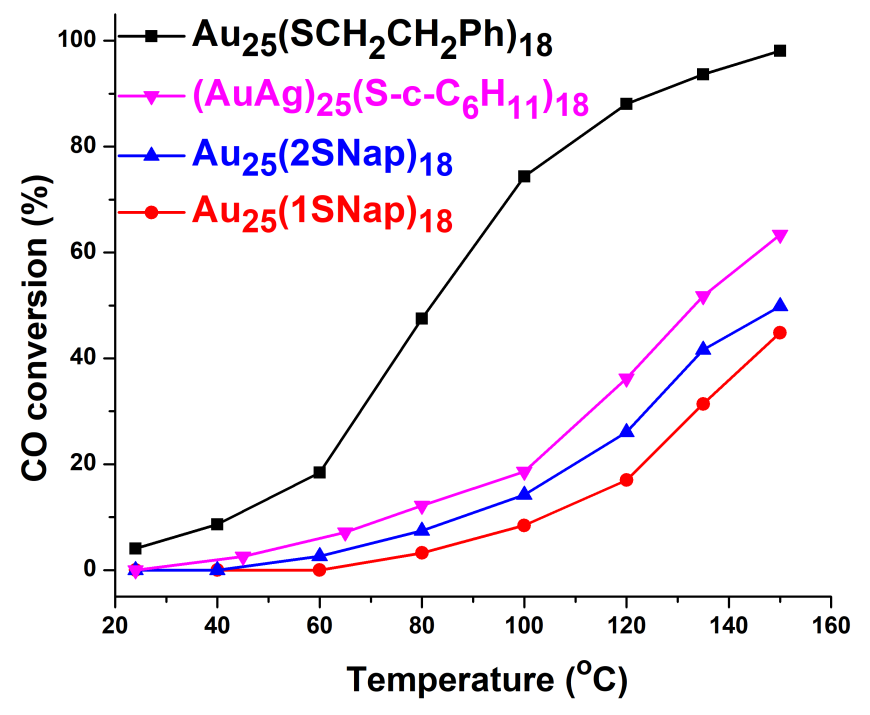

(b)

Figure 3.21: The (a) structure of $\mathrm{Au}_{25}\left(\mathrm{~S}-\mathrm{c}-\mathrm{C}_{6} \mathrm{H}_{11}\right)_{18}$ and the (b) $\mathrm{CO}$ conversion rates in the presence of $\mathrm{Ag}$ alloyed $\mathrm{Au}_{25}\left(\mathrm{~S}-\mathrm{c}-\mathrm{C}_{6} \mathrm{H}_{11}\right)_{18}$ as the catalyst. 
According to Figures 3.18 and 3.21 (b), the catalytic the CO rates of heavily doped $\mathrm{Au}_{25}$ are less than that of 5-9 Ag alloyed isomers. Figure 3.22 depicts weighted average $\Delta f$ values corresponding to 19-23 Ag alloyed isomers together with the those corresponding to unalloyed and 5-9 Ag alloyed cases. Interestingly, the majority of the outer shell sites in heavily doped $\mathrm{Au}_{25}$ have positive $\Delta f$ values. Therefore, heavy doping has caused outer shell sites to act as sites preferring to accept electrons. Due to the reason discussed under the catalytic activity of 3-5 and 5-9 Ag alloyed $\mathrm{Au}_{25}$, this huge reduction in the electron donating capability in heavily doped $\mathrm{Au}_{25}$ compared to the unalloyed cluster can be a major reason for the significant drop in the catalytic performance of heavily doped $\mathrm{Au}_{25}$. 


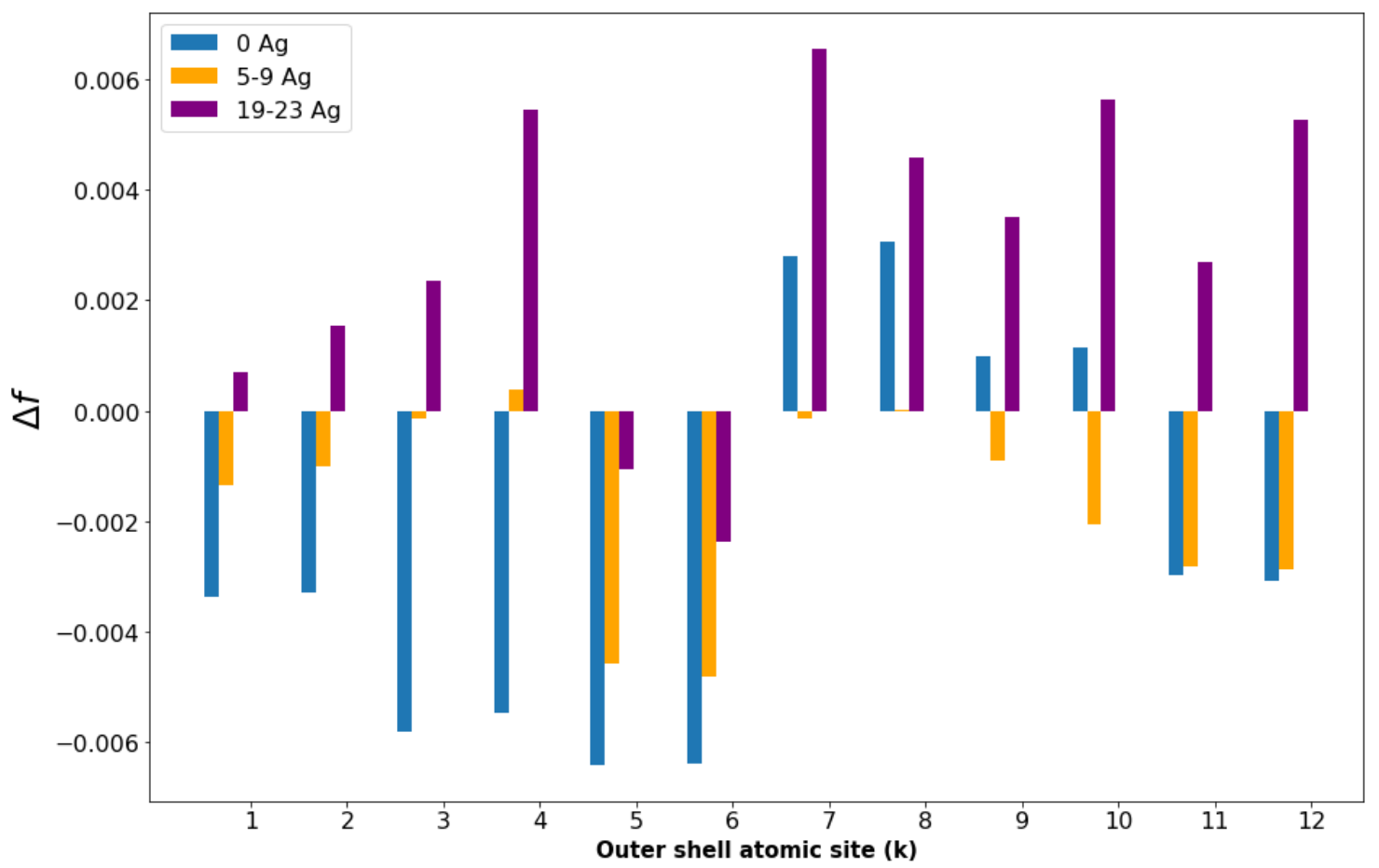

Figure 3.22: Weighted average dual descriptor values corresponding to 19-23 $\mathrm{Ag}$ alloyed $\mathrm{Au}_{25}\left(\mathrm{~S}-\mathrm{c}-\mathrm{C}_{6} \mathrm{H}_{11}\right)_{18}$ in comparison with unalloyed and 5-9 $\mathrm{Ag}$ alloyed $\mathrm{Ag}_{x} \mathrm{Au}_{25-x}\left(\mathrm{SC}_{2} \mathrm{H}_{4} \mathrm{Ph}\right)_{18}$

\subsection{Electronic and structural properties of other cluster sizes}

In this section we provide a summary of the electronic and structural properties of some selected nanoclusters.

When discussing the structural trends, we use Boltzmann weights corresponding to ten lowest energy isomers to obtain weighted average quantities. For example, the weighted average number of $\mathrm{Ag}$ atoms $n_{\text {avg }}^{A g}$ in a particular cluster is given by, 


$$
n_{\text {avg }}^{A g}=\sum_{i=1}^{10} n_{i} W_{i}
$$

where $n_{i}$ is the number of atoms in isomer $i$ and $W_{i}$ is the Boltzmann factor given by Equation. Also, for all the structures in this section, a shell metal atom is defined as the ones having exactly two bonds with $\mathrm{S}$ atoms as done in reference 165. Unless stated otherwise, all the Au atoms which do not fall in to the shell category are classified as core atoms. And I use letter $x$ to denote the number of dopant Ag atoms in a given cluster. The ligand used in all the nanoclusters in this section is $\mathrm{CH}_{3} \equiv \mathrm{R}$.

\subsection{1 $\mathrm{Au}_{18}(\mathrm{SR})_{14}$}

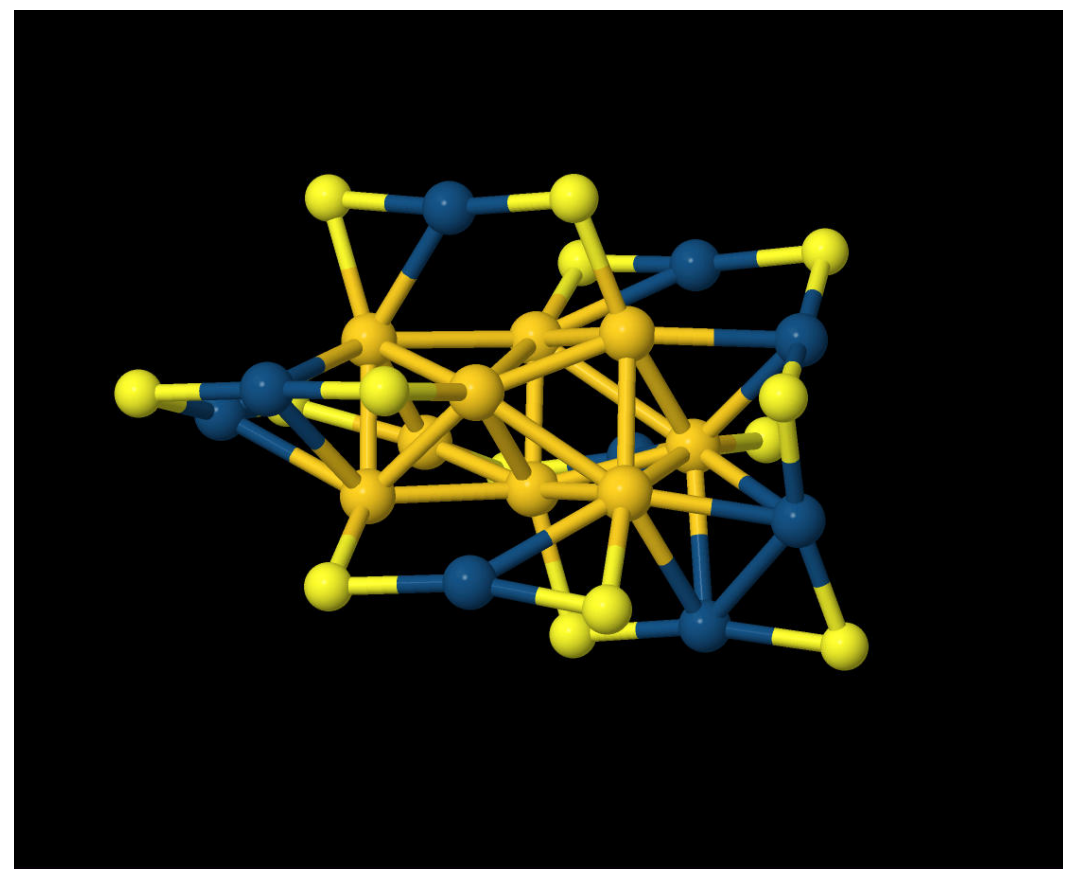

Figure 3.23: Structure of $\mathrm{Au}_{18} \mathrm{SR}_{14}$ 
In 2015, Chen et al. reported the crystal strucutre of $\mathrm{Au}_{18}(\mathrm{SR})_{14}$, making it the smallest thiolated nanocluster of which the molecular structure has been experimentally characterized ${ }^{166}$. Similar to $\mathrm{Au}_{25}(\mathrm{SR})_{18}$, the $\mathrm{Au}_{18}(\mathrm{SR})_{14}$ can be identified as a core-shell nanocluster. The 9 atom core is surrounded by 3 monomeric(SR-Au-SR), one dimeric(SR-Au-SR-Au-SR) and one tetrameric(SR-Au-SR-Au-SR-Au-SR) staple units. In Figure 3.24 we show the weighted average number of Ag atom in the core and the shell. We see that the number of Ag atoms in the core increases with the total number of $\mathrm{Ag}$ atoms(x) in the cluster and plateaus when $\mathrm{x}=12$. The number of $\mathrm{Ag}$ atoms in the core at this point is about 6 . For $\mathrm{x}<12$, the majority of the Ag atoms is in the core. When $\mathrm{x}$ is increased beyond 12 , shell sites started to get predominantly occupied by $\mathrm{Ag}$ silver atoms. It is interesting to note that even when $\mathrm{x}=17$, not all the core sites are substituted with $\mathrm{Ag}$ and for $\mathrm{x}>14$, the preferable Ag dopant sites seem to be in the shell. 


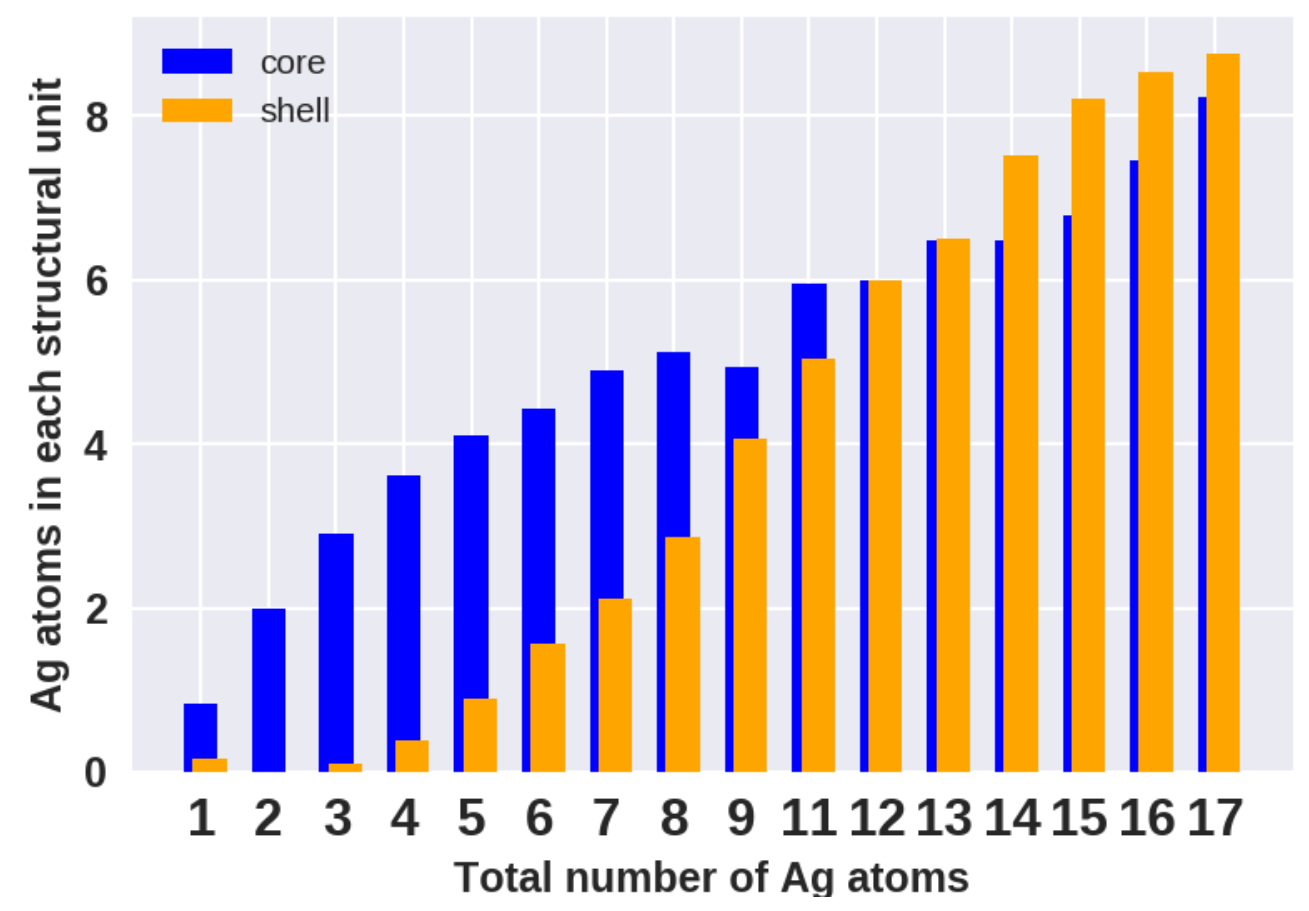

Figure 3.24: Structural trends in $\mathrm{Ag}$ alloyed $\mathrm{Au}_{18} \mathrm{SR}_{14}$

The average DOS shown in Figure 3.25 indicates that Ag doping affects both HOMO and and LUMO states. Compared to LUMO, HOMO states show greater broadening due to $\mathrm{Ag}$ doping. The experimental absorption spectra obtained by Chen et al. shows features at $670 \mathrm{~nm}, 580 \mathrm{~nm}, 480 \mathrm{~nm}$, and $380 \mathrm{~nm}^{166}$. Our HOMOLUMO gap, which is about 1.7 ev closely matches with the energy corresponding to the peak at $670 \mathrm{~nm}(1.85 \mathrm{eV})$. With increasing $\mathrm{x}$, a consistent shift of the HOMO level energy to higher energies and a drop in the HOMO level electron density is observed. Thus, we predict a drop in the HOMO-LUMO photon absorption intensity as the number of $\mathrm{Ag}$ atoms in the system increases. 


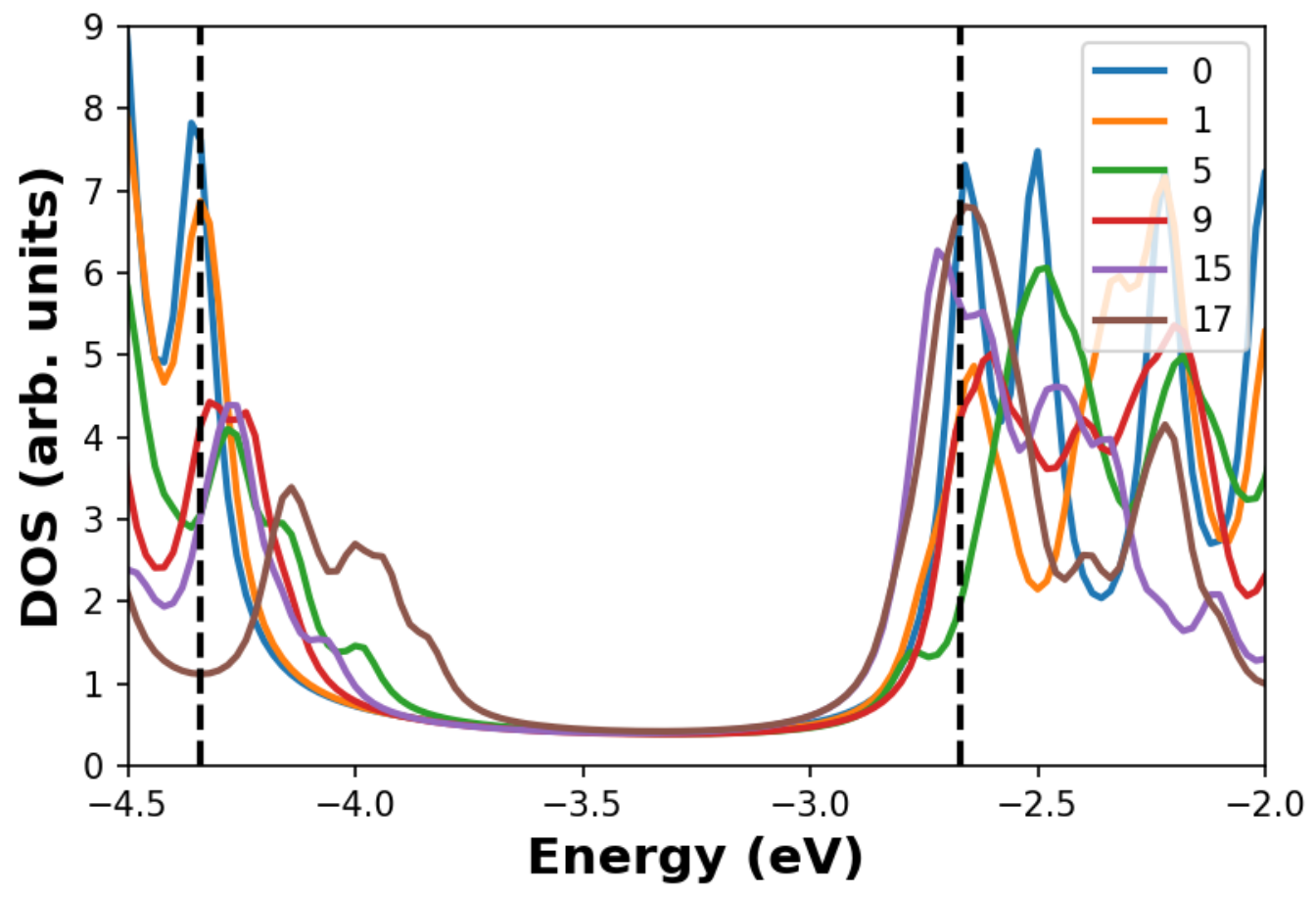

Figure 3.25: Density of states of $\mathrm{Ag}$ alloyed $\mathrm{Au}_{18} \mathrm{SR}_{14}$ 


\subsection{2 $\quad \mathrm{Au}_{20}(\mathrm{SR})_{16}$}

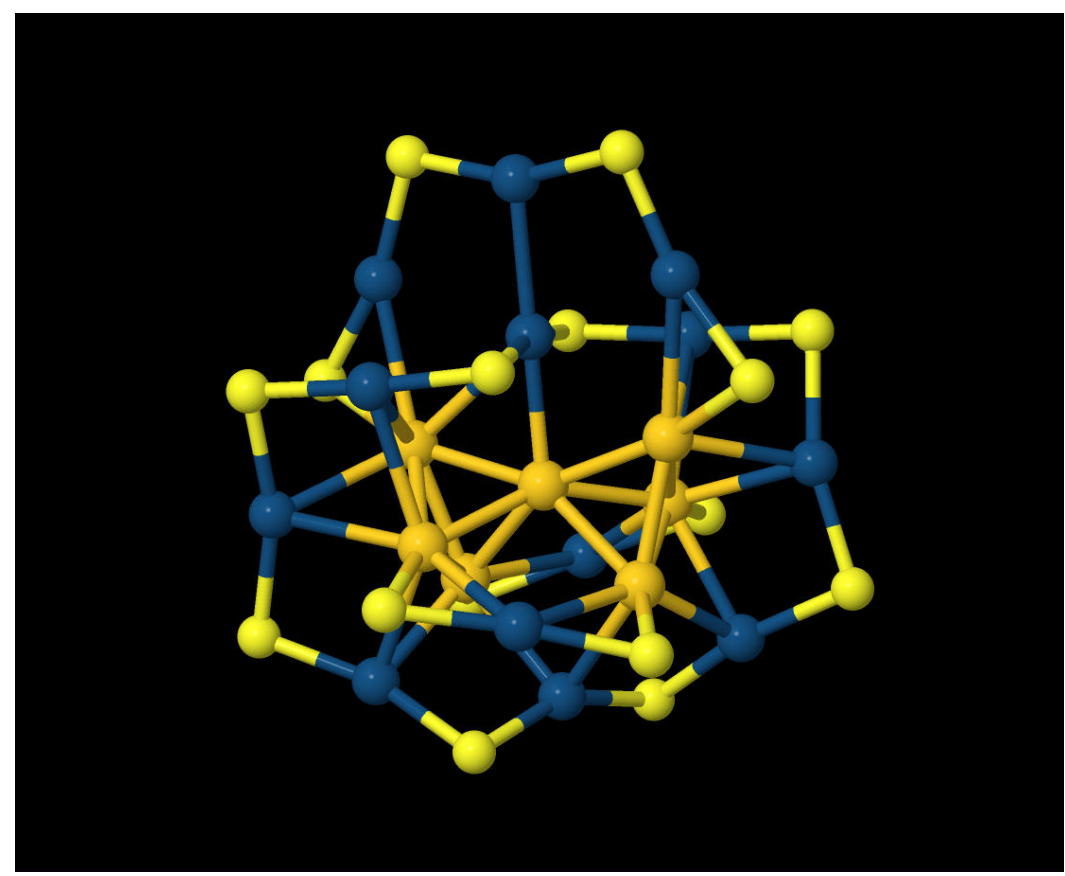

Figure 3.26: Structure of $\mathrm{Au}_{20} \mathrm{SR}_{16}$

The crystal structure of $\mathrm{Au}_{20}(\mathrm{SR})_{16}$ was predicted by Pei at al. and Jiang et al. ${ }^{167,168}$. However the experimentally obtained structure by Jin et al. in 2014 with SPh- $t \mathrm{Bu}$ as the ligand was quite different from these theoretically predicted ones ${ }^{169}$. This nanocluster comprises of a 7 -atom gold core surrounded by a $\mathrm{Au}_{8}(\mathrm{SR})_{8}$ ring (which was observed for the first time in a nanocluster) and monomeric and trimeric staple units. Accordingly there are 13 atoms in the shell. Interestingly, bonds connecting $\mathrm{S}$ and the core $\mathrm{Au}$ atoms were not observed in this cluster. However strong $\mathrm{Au}_{\text {ring }}-\mathrm{Au}_{\text {core }}$ bonds are believed to stabilize its structure. Jin et al. further showed that $\mathrm{Au}_{20}(\mathrm{SR})_{16}$ can be thought of as the basic structure of a series - $\mathrm{Au}_{20}(\mathrm{SR})_{16}, \mathrm{Au}_{28}(\mathrm{SR})_{20}, \mathrm{Au}_{36}(\mathrm{SR})_{24}$, 
$\mathrm{Au}_{40}(\mathrm{SR})_{28}$ — which evolves with the addition of $\mathrm{Au}_{8}(\mathrm{SR})_{4}$ units. The number of electrons in this series increases as 4,8,12 and 16 respectively. Thus, these nanoclusters do not comply with the superatom theory.

According to our calculations (Figure 3.27) the number of Ag atoms in the core increases with the increasing number of total $\mathrm{Ag}$ in the system. However, even when $\mathrm{x}=18$, when the cluster is predominantly doped with $\mathrm{Ag}$, the core sites are not being fully occupied by $\mathrm{Ag}$. Similar to $\mathrm{Au}_{18}(\mathrm{SR})_{14}$, the shell sites happen to be the most favorable dopant locations when the cluster is heavily doped with Ag.

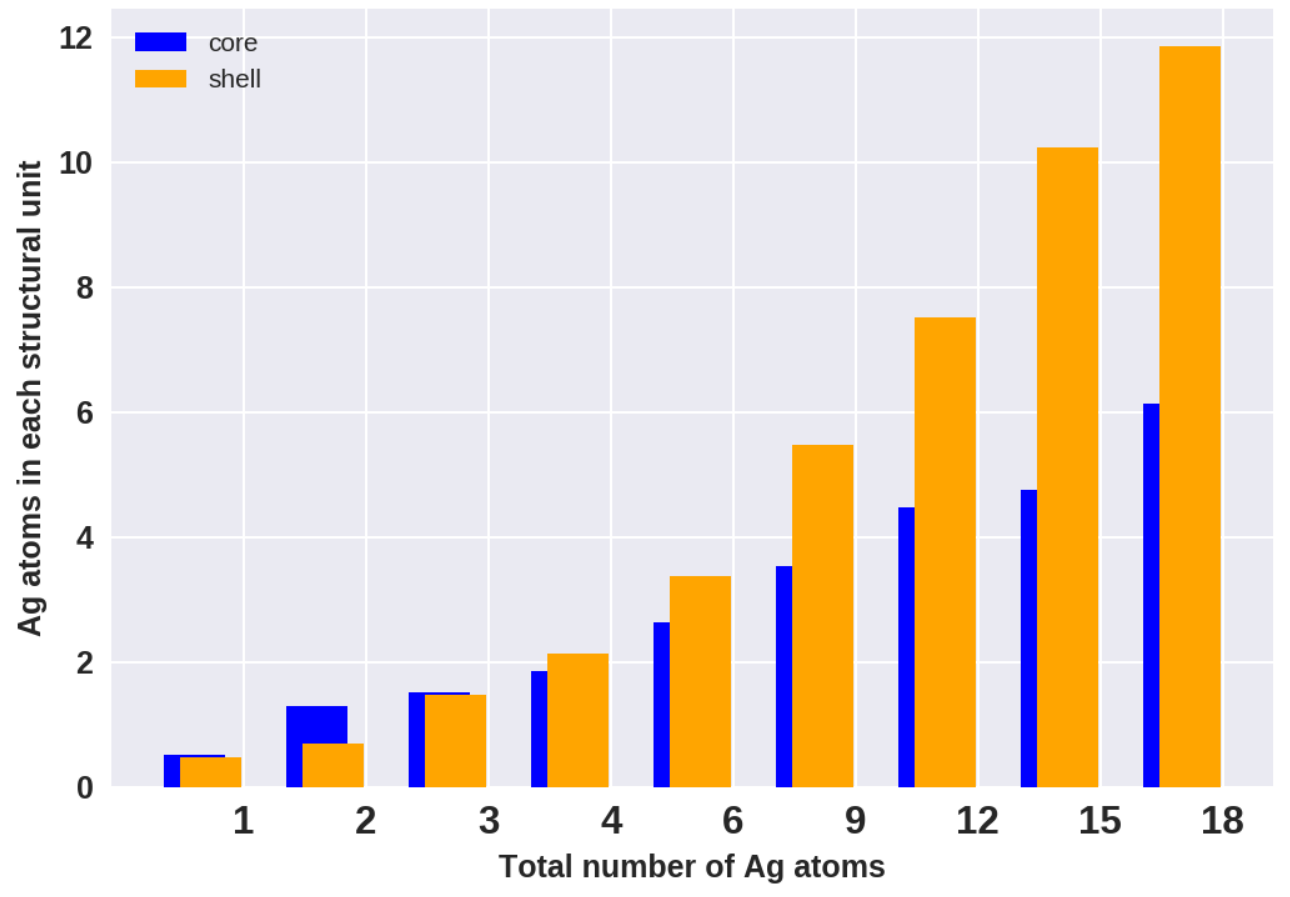

Figure 3.27: Structural trends in $\mathrm{Ag}$ alloyed $\mathrm{Au}_{20} \mathrm{SR}_{16}$ 
Also as we saw in the case of $\mathrm{Au}_{18}(\mathrm{SR})_{18}, \mathrm{HOMO}$ states of $\mathrm{Au}_{20}(\mathrm{SR})_{16}$ are significantly affected by Ag doping, except when the number of dopants are small. The LUMO states are not greatly affected except for a slight broadening. Therefore it is likely that the HOMO-LUMO gap of this cluster cannot be changed by doping with one or two Ag atoms.

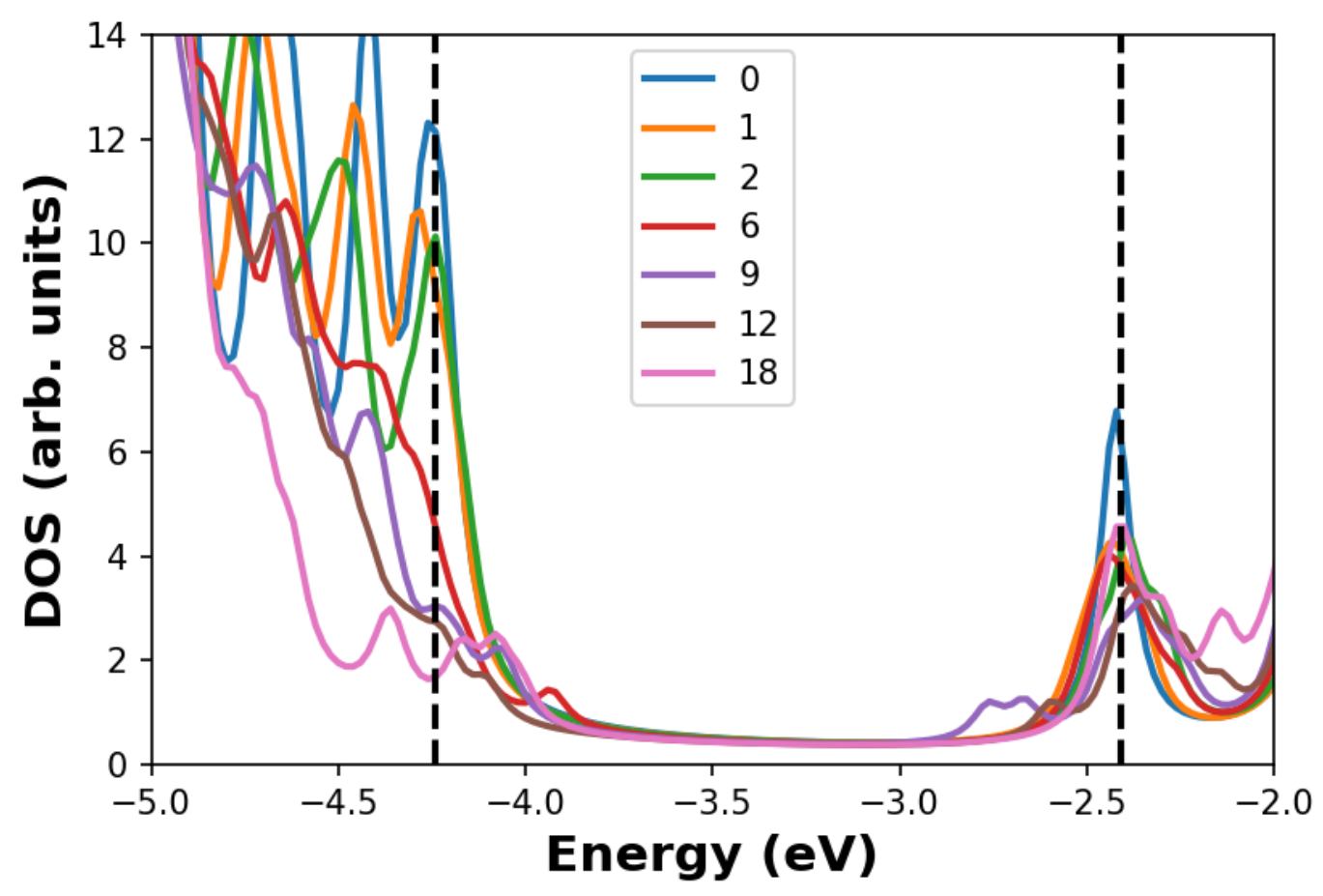

Figure 3.28: Density of states of $\mathrm{Ag}$ alloyed $\mathrm{Au}_{20} \mathrm{SR}_{16}$ 


\subsection{3 $\mathrm{Au}_{24}(\mathrm{SR})_{20}$}

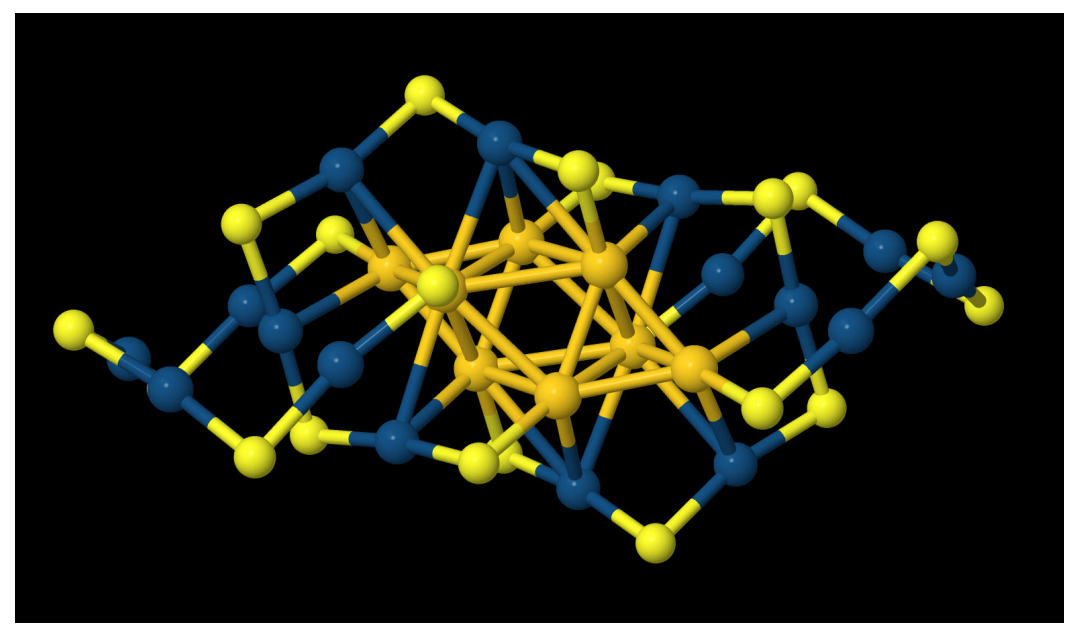

Figure 3.29: Structure of $\mathrm{Au}_{24} \mathrm{SR}_{20}$

In 2009, Jin et al. reported the synthesis of $\mathrm{Au}_{24}(\mathrm{SR})_{20}$ through one-pot for onestructure process ${ }^{170}$. This is another structure of which the stability cannot be explained with the superatom theory. The purified $\mathrm{Au}_{24}(\mathrm{SR})_{20}$ cluster exhibits a main absorption peak at $765 \mathrm{~nm}$ which corresponds to the HOMO-LUMO gap and another shoulder band at $400 \mathrm{~nm}$. The structural trends in $\mathrm{Ag}$ alloyed $\mathrm{Au}_{24}(\mathrm{SR})_{20}$ are similar to those of $\mathrm{Au}_{20}(\mathrm{SR})_{16}$. There are 8 core sites and 16 outer shell sites in $\mathrm{Au}_{24}(\mathrm{SR})_{20}$. The Ag population in the shell gradually increases as $x$ increases. When $x=9$, both the core and the shell seem to contain equal amounts of Ag atoms. The core gets saturated with $\mathrm{Ag}$, when the total number of $\mathrm{Ag}$ is 21 . 


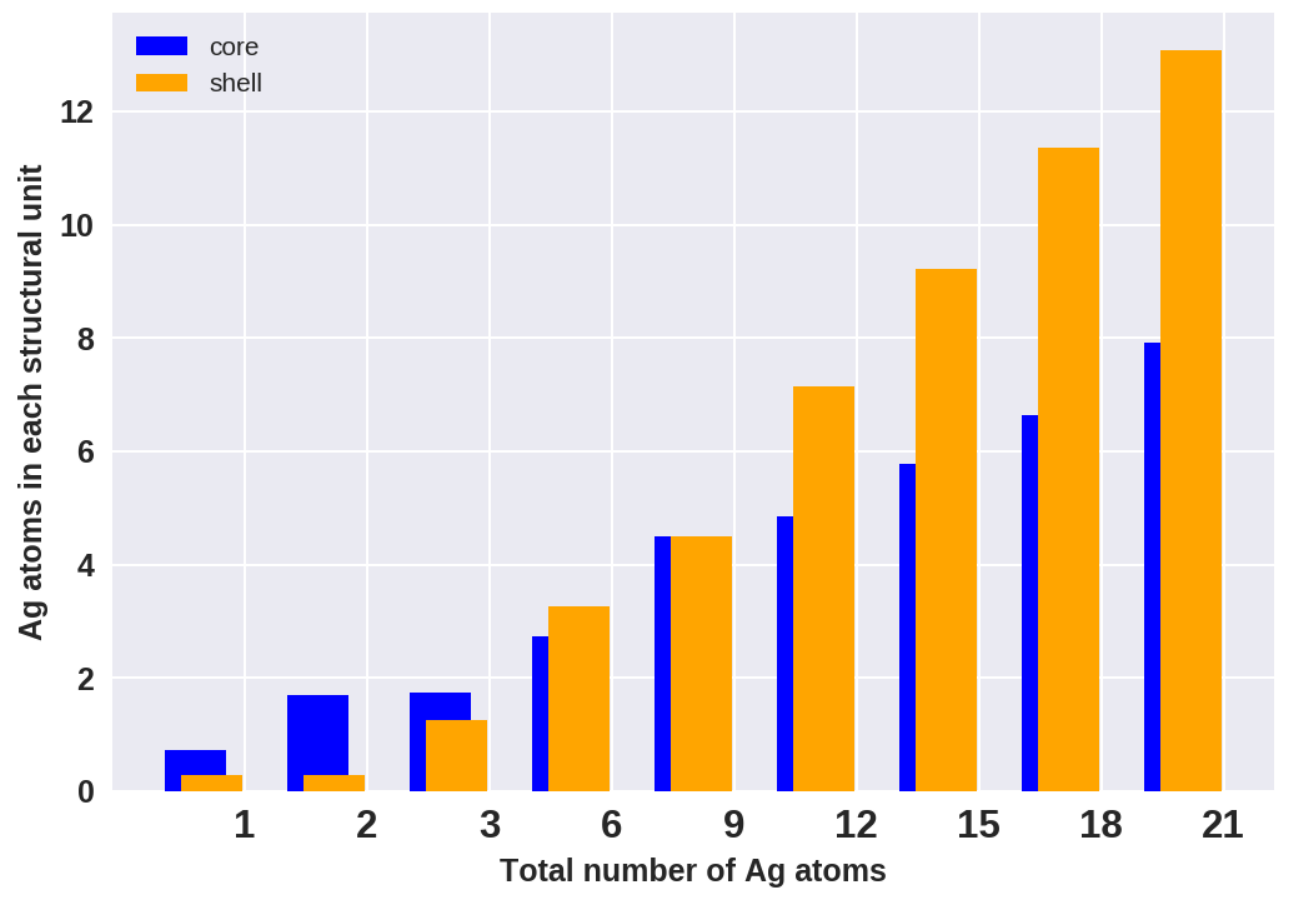

Figure 3.30: Structural trends in $\mathrm{Ag}$ alloyed $\mathrm{Au}_{24} \mathrm{SR}_{20}$

Unalloyed $\mathrm{Au}_{24}(\mathrm{SR})_{20}$ cluster posses a HOMO-LUMO gap of about $2.0 \mathrm{eV}$. As the number of $\mathrm{Ag}$ atoms in the system increases, the HOMO level tends to shift to lower energies. Shifts in the LUMO level on the other hand are not uniform. For $\mathrm{x}=1$ and $\mathrm{x}=6$, LUMO level is slightly shifted toward lower energies while for $\mathrm{x}>12$, the shift in the LUMO level is toward higher energies. 


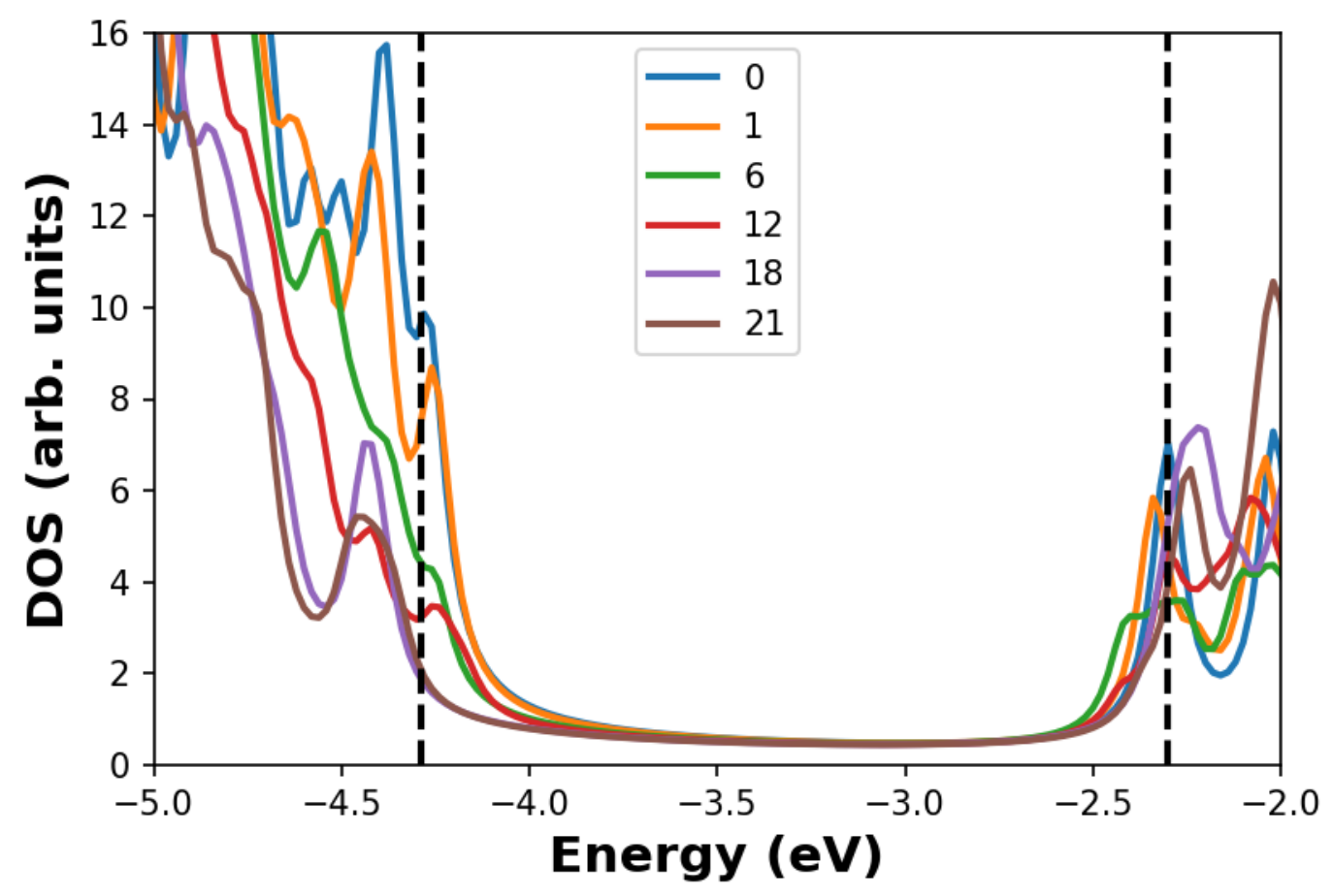

Figure 3.31: Density of states of $\mathrm{Ag}$ alloyed $\mathrm{Au}_{24} \mathrm{SR}_{20}$

\subsection{4 $\mathrm{Au}_{30}(\mathrm{SR})_{18}$}

Crystal structure of $\mathrm{Au}_{30}(\mathrm{SR})_{18}$ was independently resolved by Dass et al. and Jin eat al. using S-tBu and S-Adm as the protecting ligands respectively ${ }^{171,172}$. The core and shell consists of 17 and 13 atoms respectively. Contrary to the the trends in the Ag location for other nanoclusters with the number of Au atoms less than 30, the most preferable dopant locations in $\mathrm{Au}_{30}(\mathrm{SR})_{18}$ remain to be in the core, even for heavy doping. However, the number of Ag atoms in both core and shell progressively increases with the increasing number of dopants introduced to the system. 


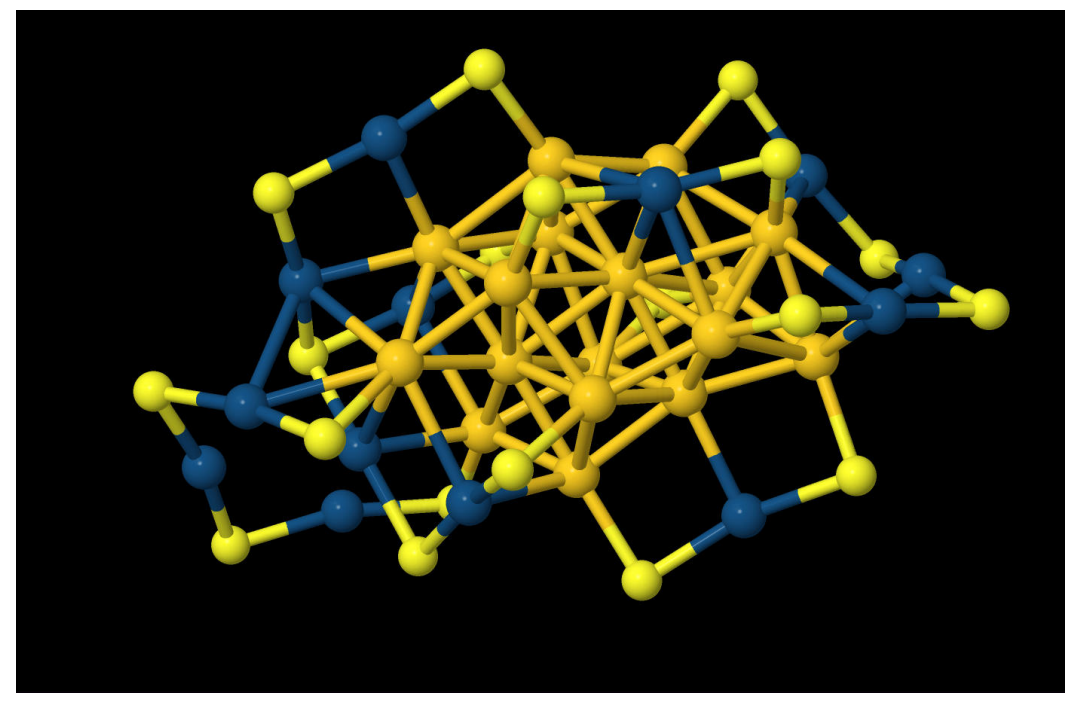

Figure 3.32: Structure of $\mathrm{Au}_{30} \mathrm{SR}_{18}$ 


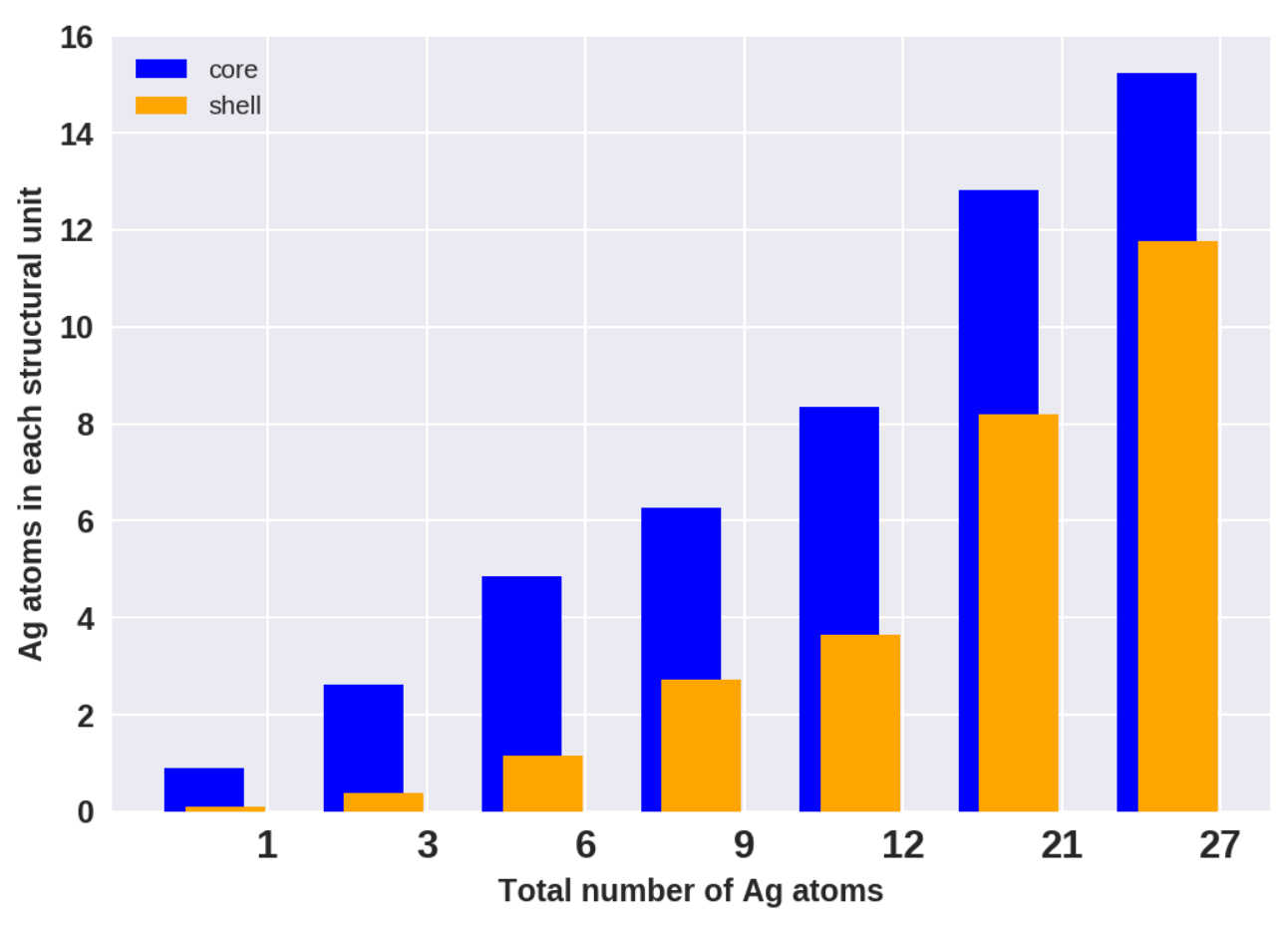

Figure 3.33: Structural trends in $\mathrm{Ag}$ alloyed $\mathrm{Au}_{30} \mathrm{SR}_{18}$

The HOMO-LUMO gap is about $1.2 \mathrm{eV}$. The energies of the frontier molecular orbitals are not affected for small amounts of Ag doping, The shifts in the HOMO and LUMO level energies are observed for $\mathrm{x}>9$ and with heavy doping, a significant shift in the LUMO level toward lower energies can be seen. 


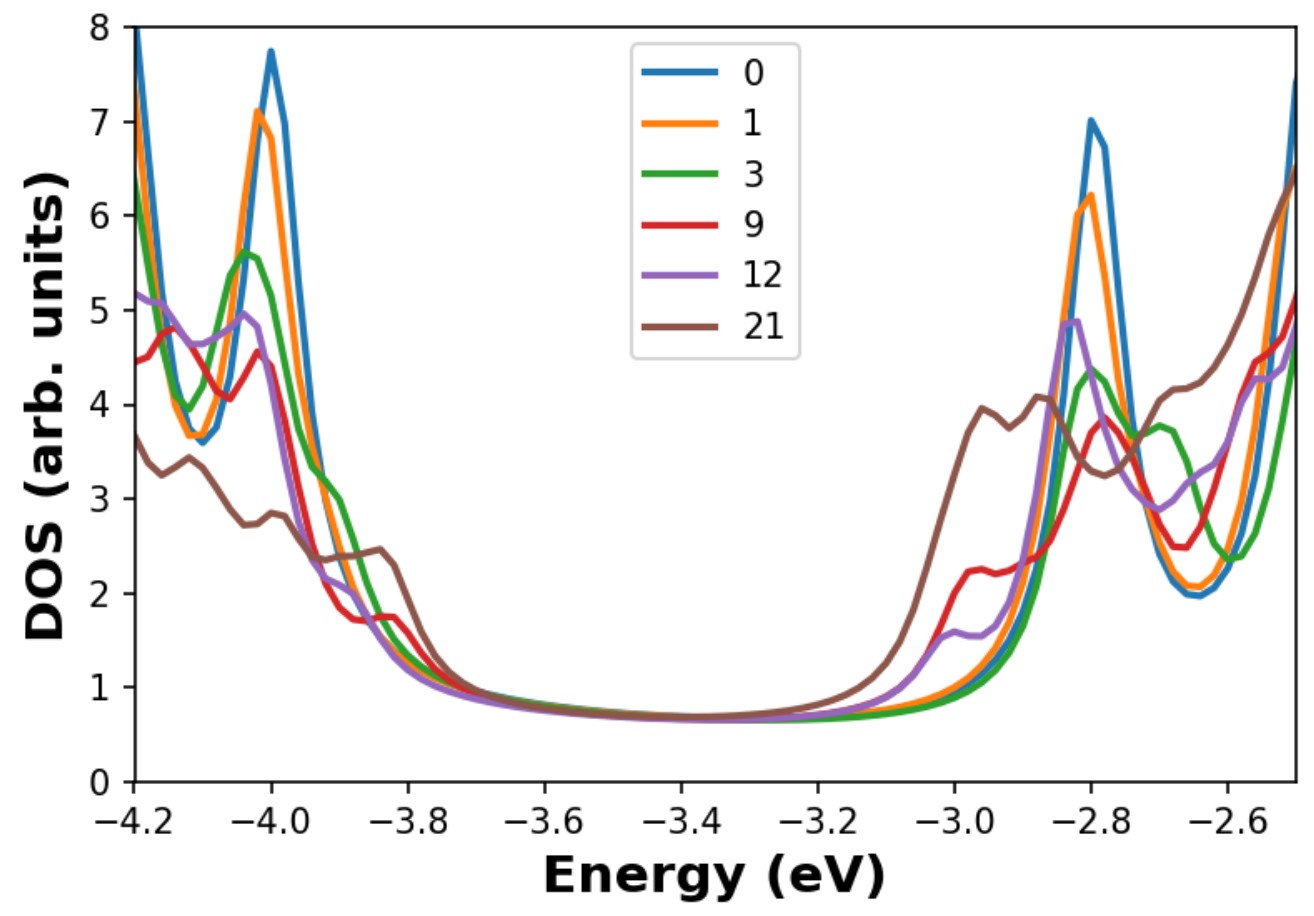

Figure 3.34: Density of states of $\mathrm{Ag}$ alloyed $\mathrm{Au}_{30} \mathrm{SR}_{18}$ 


\subsection{5 $\quad \mathrm{Au}_{36} \mathrm{SR}_{24}$}

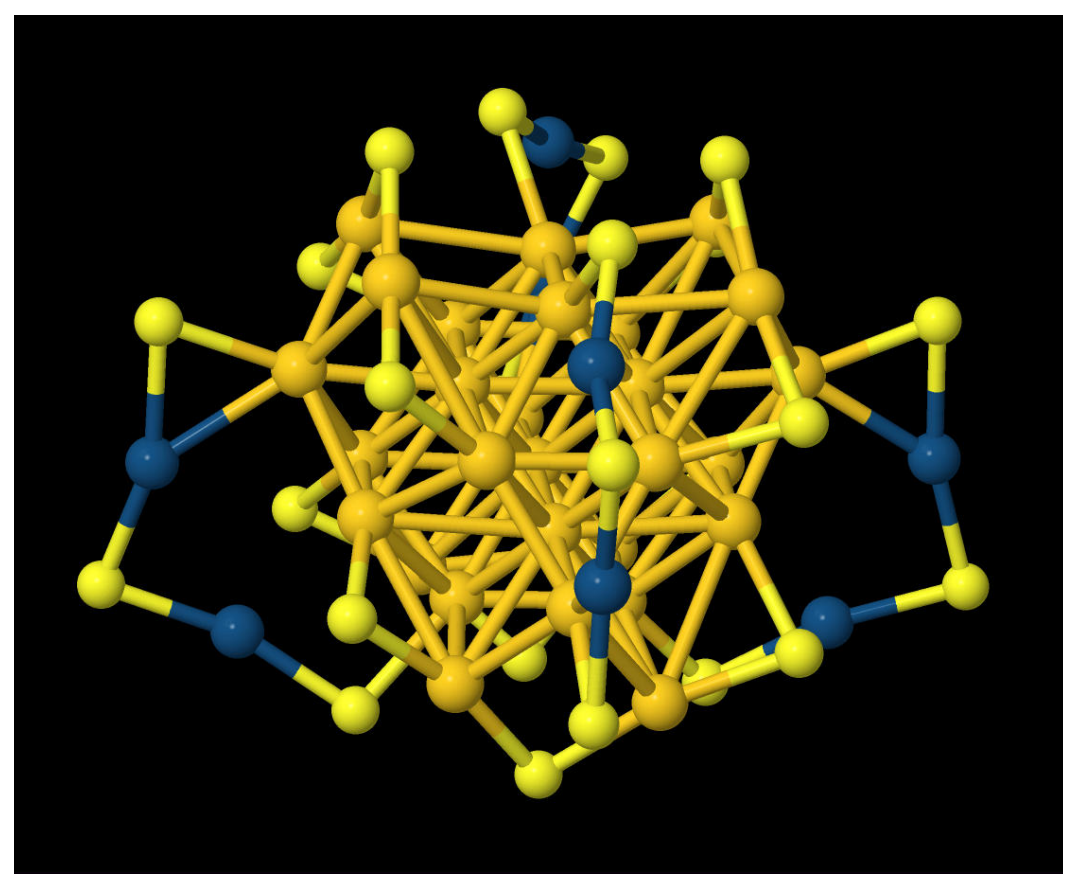

Figure 3.35: Structure of $\mathrm{Au}_{36} \mathrm{SR}_{24}$

Jin et al. reported the discovery of $\mathrm{Au}_{36}(\mathrm{SR})_{24}$ nanoparticle with $\mathrm{SPh}-\mathrm{tBu}$ as the ligand in $2012^{173}$. This nanoparticle was synthesized by reacting $\mathrm{Au}_{38}\left(\mathrm{SCH}_{2} \mathrm{CH}_{2} \mathrm{Ph}\right)_{24}$ with $\mathrm{HSPh}-\mathrm{tBu}$. The core of the nanoparticle consists of 28 gold atoms arranged in form resembling the FCC crystal structure. The other 8 gold atoms form 4 dimeric staples each consisting of 3 sulfur atoms. They also report a HOMO-LUMO gap of $1.5 \mathrm{eV}$ for the DFT optimized structure. In another study, Jin et al. examined the bonding properties of this nanoparticle and showed that $\mathrm{Au}_{28}$ core contains pseudo$\mathrm{Au}_{4}$ units with strong $\mathrm{Au}-\mathrm{Au}$ bonds ${ }^{174}$. There has been no reported work on doping the $\mathrm{Au}_{36}(\mathrm{SR})_{24}$ system. Our calculations show that the preferable Ag dopant sites are 
in the core of $\mathrm{Au}_{36}(\mathrm{SR})_{24}$ even when the number of dopants are as high as 24. This is not a surprising result as out of the $36 \mathrm{Au}$ sites only 8 have classified as belonging to the shell. The presence of Ag in the staple units is only visible for number of dopants greater than $\sim 12$.

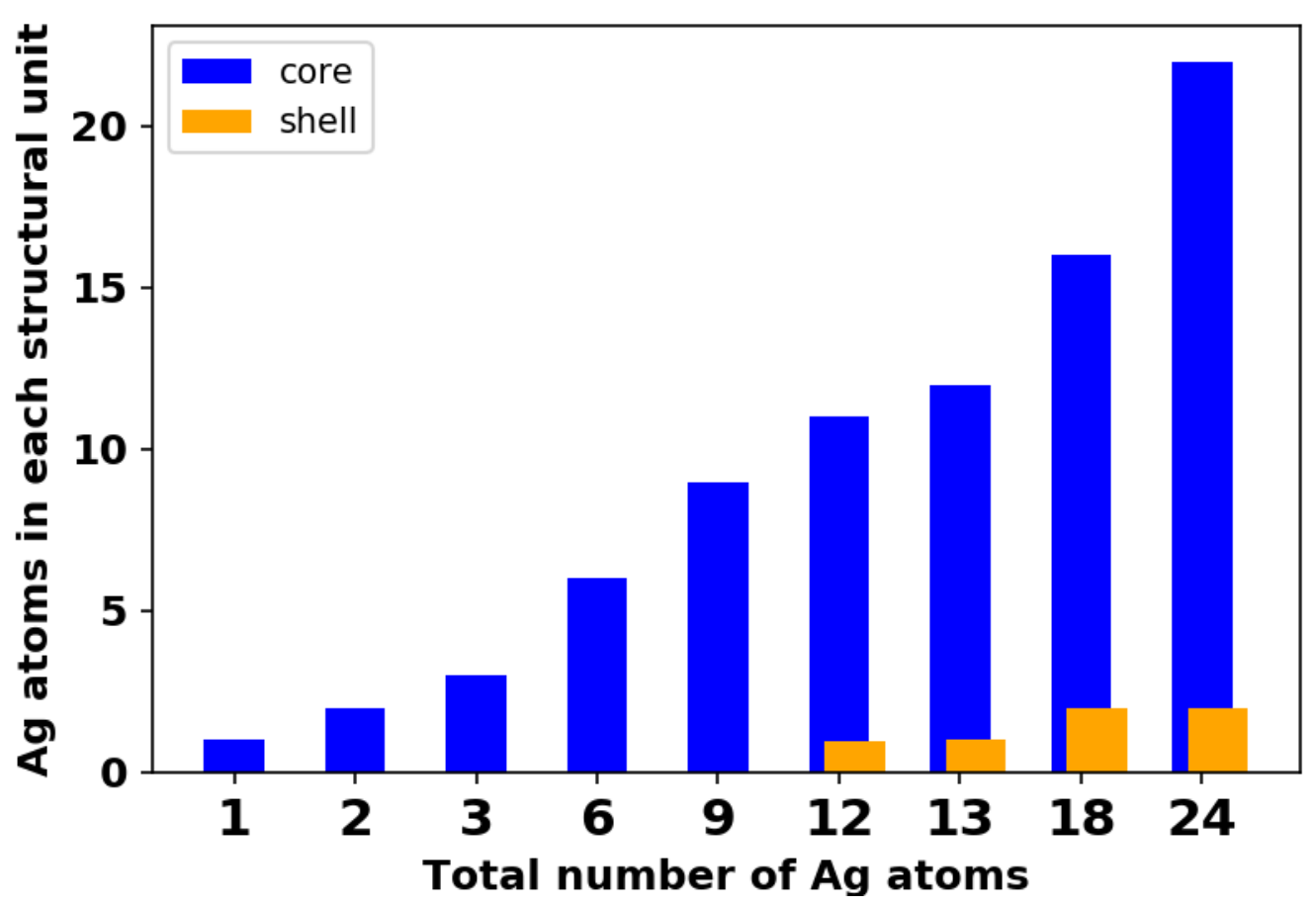

Figure 3.36: Density of states of $\mathrm{Ag}$ alloyed $\mathrm{Au}_{36}(\mathrm{SR})_{24}$

Interestingly, the LUMO states of $\mathrm{Au}_{36}(\mathrm{SR})_{24}$ are the least affected due to Ag doping when compared with the DOS of the other nanoclusters considered in this section. A major reason for this could be the availability of a large number of dopant sites in the core of $\mathrm{Au}_{30}$. Therefore even with heavy doping, the structure of $\mathrm{Au}_{30}$ is not that affected, as most of the $\mathrm{Ag}$ atoms occupy the core, which is stabilized by 
the protecting ligands. However, for $\mathrm{x}>6$ we see that both HOMO and LUMO states are broadened as in the case of other nanoclusters. The HOMO-LUMO gap of the unalloyed cluster is about $1.76 \mathrm{eV}$. The shifts in the frontier molecular orbital energies due to Ag doping are negligible. However, because of the considerable broadening in these states, we expect to see an increase in the photon absorption intensity with the increase in the amount of $\mathrm{Ag}$ in the system.

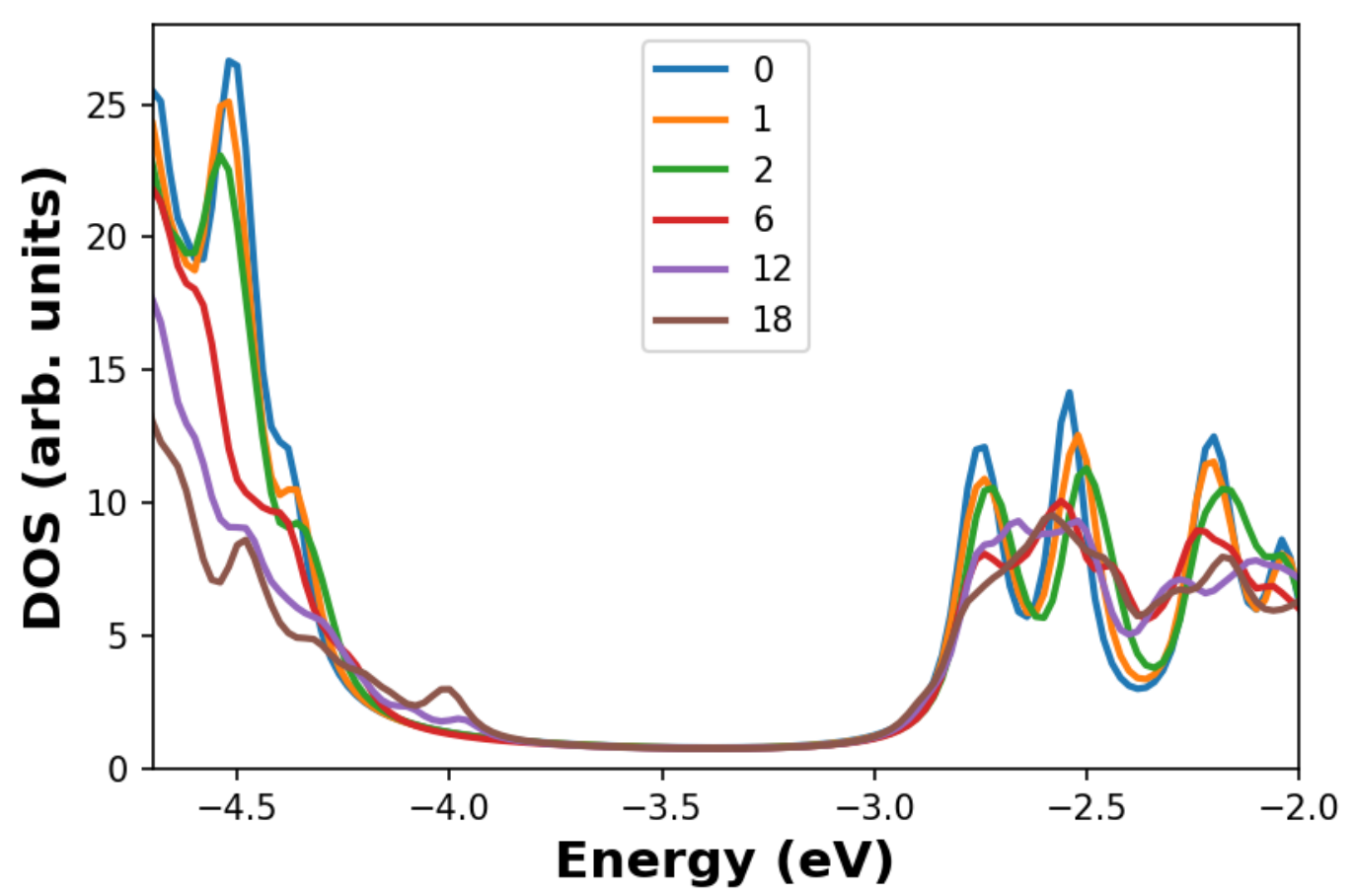

Figure 3.37: Density of states of $\mathrm{Ag}$ alloyed $\mathrm{Au}_{36}(\mathrm{SR})_{24}$ 


\subsection{6 $\quad \mathrm{Au}_{38}(\mathrm{SR})_{24}$}

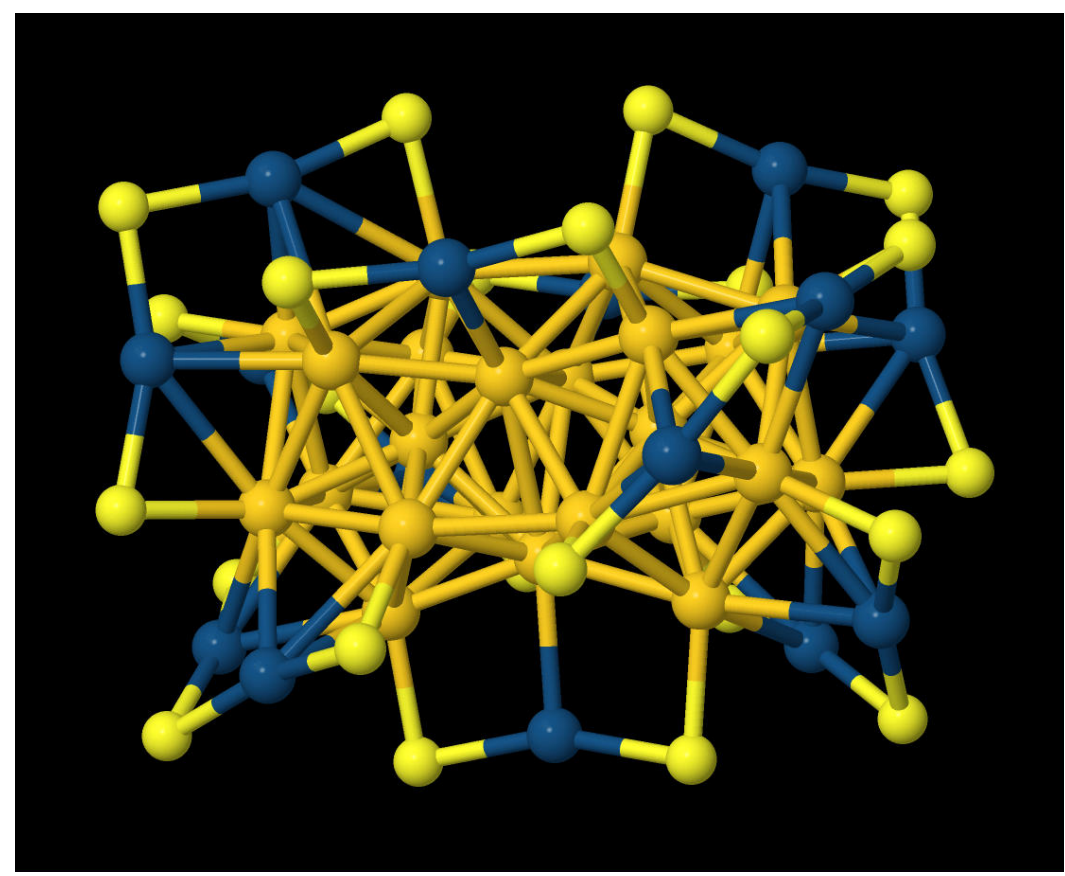

Figure 3.38: Structure of $\mathrm{Au}_{38} \mathrm{SR}_{24}$

Crystal structure of $\mathrm{Au}_{38}(\mathrm{SR})_{24}$ was first determined by Qian et al. by X-ray crystallography in $2010^{175}$. Prior to the experimental determination, two predictions on the structure of $\mathrm{Au}_{38}(\mathrm{SR})_{24}$ were made by Pei et al. and Lopez-Acevedo et al. ${ }^{176,177}$. While both these predictions are similar, the one by Lopez-Acevedo was the closest to the experimental structure. This is also a core-shell nanoparticle with core consisting of 23 gold atoms. The structure of the core, can be viewed as formed by the fusion of two $\mathrm{Au}_{13}$ units by sharing three $\mathrm{Au}$ atoms in a triangular facet of one of the $\mathrm{Au}_{13}$ unit. This core is stabilized by three monomeric and six dimeric staple-like units. LopezAcevedo et al. also showed that the electronic structure of $\mathrm{Au}_{38}$ could be explained 
using the particle in a cylinder model. ${ }^{177}$ This has a large HOMO-LUMO gap. Jung et al. tested the stability of $\mathrm{Au}_{38}(\mathrm{SR})_{24}$ for the ligands $\mathrm{CH}_{3}, \mathrm{C}_{6} \mathrm{H}_{13}, \mathrm{CH}_{2} \mathrm{CH}_{2} \mathrm{Ph}, \mathrm{Ph}$, $\mathrm{PhF}$, and $\mathrm{PhCOOH} .{ }^{178}$ Negishi found that $\mathrm{Au}_{38}(\mathrm{SR})_{14}$ can be doped with up to two $\operatorname{Pd}$ atoms $^{36}$.

Similar to $\mathrm{Au}_{30}(\mathrm{SR})_{18}$ cluster, preferable dopant sites are in the core for all the doping levels considered. However, as it is the case with other thiolated nanoclusters, occupancy of core and shell sites by Ag consistently increases with the increasing number of dopants. It should also be noted that both in $\mathrm{Au}_{30}(\mathrm{SR})_{18}$ and $\mathrm{Au}_{38}(\mathrm{SR})_{24}$, even when there is only one $\mathrm{Ag}$ atom, there is a probability for it to occupy a shell site. This is somewhat surprising given that both these structures prefer to keep the dopants in the core even with heavy doping. 


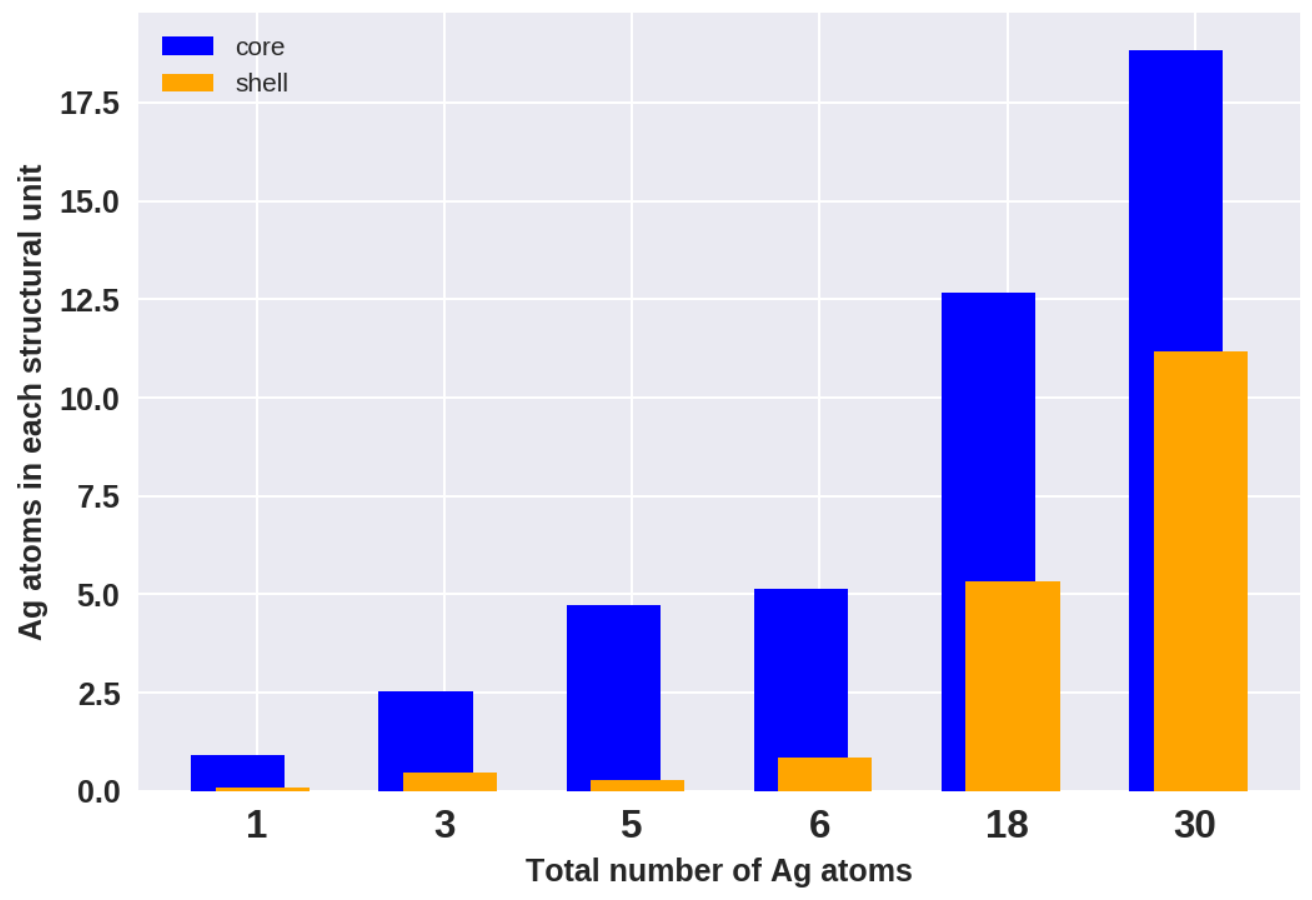

Figure 3.39: Structural trends in $\mathrm{Ag}$ alloyed $\mathrm{Au}_{38} \mathrm{SR}_{24}$

As it is the case with most of the thiolated clusters we examined so far, the electronic properties of frontier molecular orbitals of $\mathrm{Au}_{38}(\mathrm{SR})_{24}$ are not affected due to small amounts of $\mathrm{Ag}$, as indicated by the similar shapes of the DOS peaks corresponding to $\mathrm{HOMO}$ and LUMO states. However, starting from $\mathrm{x}=3$, HOMO and LUMO states are shifted toward higher and lower energies respectively, resulting in a smaller HOMO-LUMO gap. Despite for the fact that the HOMO state of 100 $\% \mathrm{Ag}$ doped structure exhibits the greatest shift, its LUMO state is only slightly shifted compared to the unalloyed structure. Another interesting feature is that the 
densities of both HOMO and LUMO states gradually decrease as the number of Ag atoms increases.

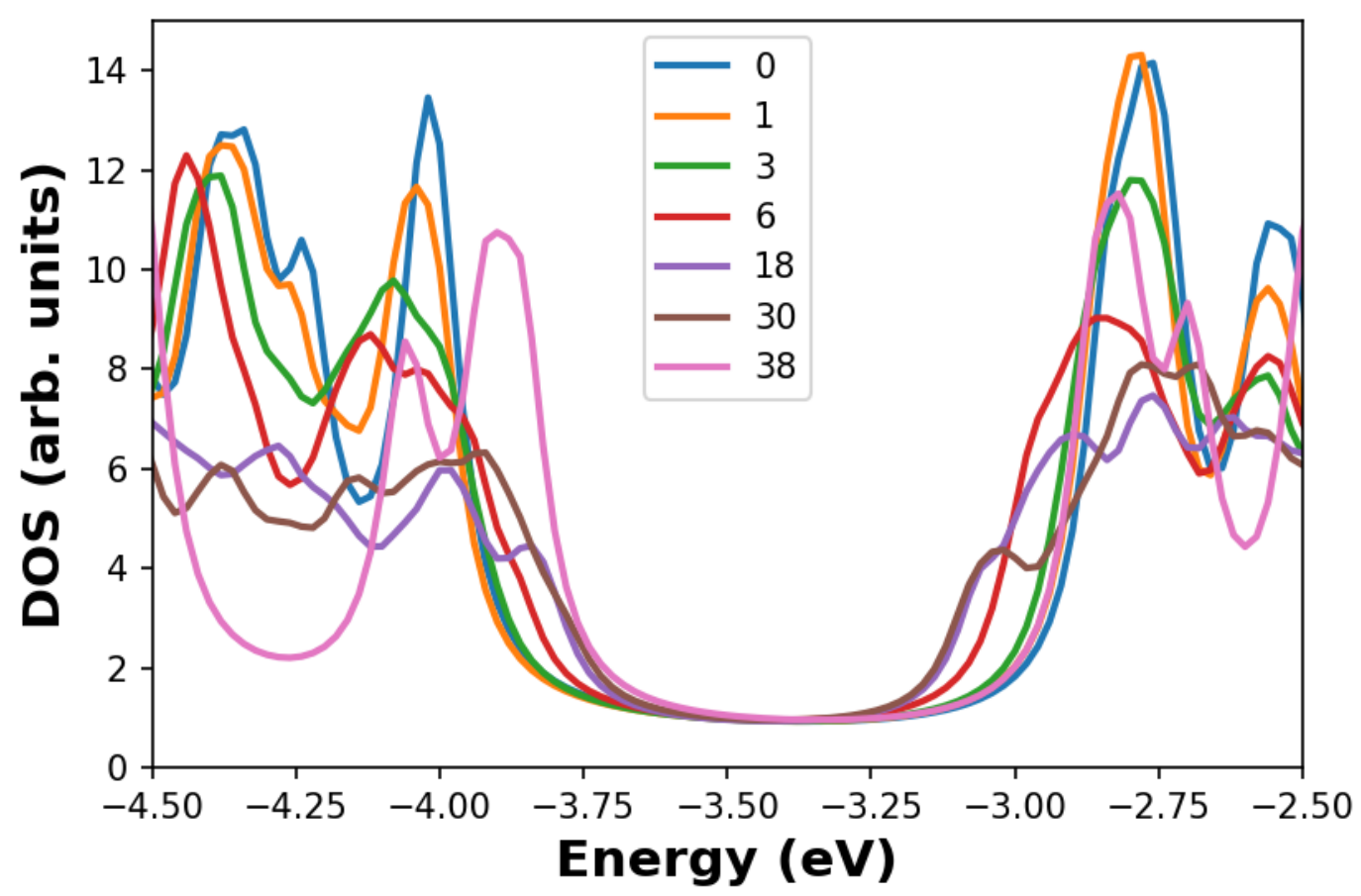

Figure 3.40: Density of states of $\mathrm{Ag}$ alloyed $\mathrm{Au}_{38} \mathrm{SR}_{24}$

\subsection{7 $\quad \mathrm{Au}_{102} \mathrm{SR}_{44}$}

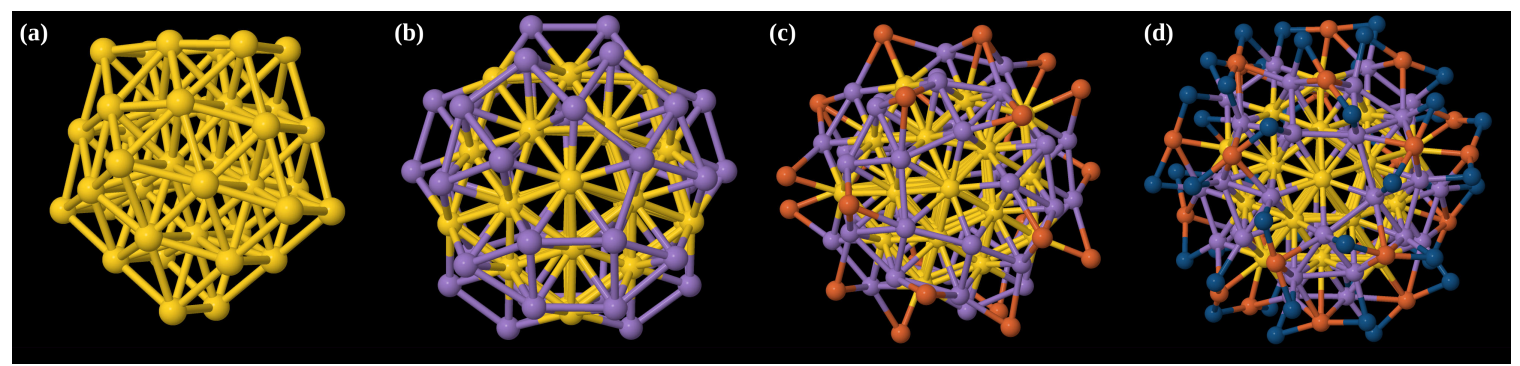

Figure 3.41: Structure of $\mathrm{Au}_{102} \mathrm{SR}_{44}$ 
$\mathrm{Au}_{102} \mathrm{SR}_{44}$ is the first thiolated nanocluster of which the structure was experimentally determined. ${ }^{27}$ This structure consists of three shells with 39,40 and 23 gold atoms respectively. The shell with 23 gold atoms interact with 44 monomeric staples each consisting of one Au atom and two sulfur atoms. Gao et al. and Li et al. showed using DFT calculations that $\mathrm{Au}_{102} \mathrm{SR}_{44}$ cluster has a HOMO-LUMO gap of about $0.5 \mathrm{eV}^{179,180}$. The ligands in the staple units have significant effect on this gap as the DOS of bare $\mathrm{Au}_{102} \mathrm{SR}_{44}$ without ligands shows a gap smaller than $0.16 \mathrm{eV}$. Later experimental/theoretical work by Hulkko et al. also confirmed the existence of the HOMO-LUMO transition with $0.5 \mathrm{eV}$ of energy ${ }^{181}$. Gao et al. studied the ligand effects in $\mathrm{Au}_{102}(\mathrm{p}-\mathrm{MBA})_{40}(\mathrm{p}-\mathrm{BBT})_{4}$ and suggested $\left.\left(\mathrm{CH}_{3}\right)_{2}-\mathrm{C}_{6} \mathrm{H}_{4}-\mathrm{SH}\right)$ and para-amino benzenethiol $\left(\mathrm{NH}_{2}-\mathrm{C}_{6} \mathrm{H}_{4}-\mathrm{SH}\right)$ as favorable candidates over p-BBT.

Our calculations show that in $\mathrm{Au}_{102} \mathrm{SR}_{44}, \mathrm{Ag}$ atoms prefer to occupy the innermost core even with heavy doping. The Ag occupancy in two outer shells also seem to increase linearly as the number of dopants increases. 


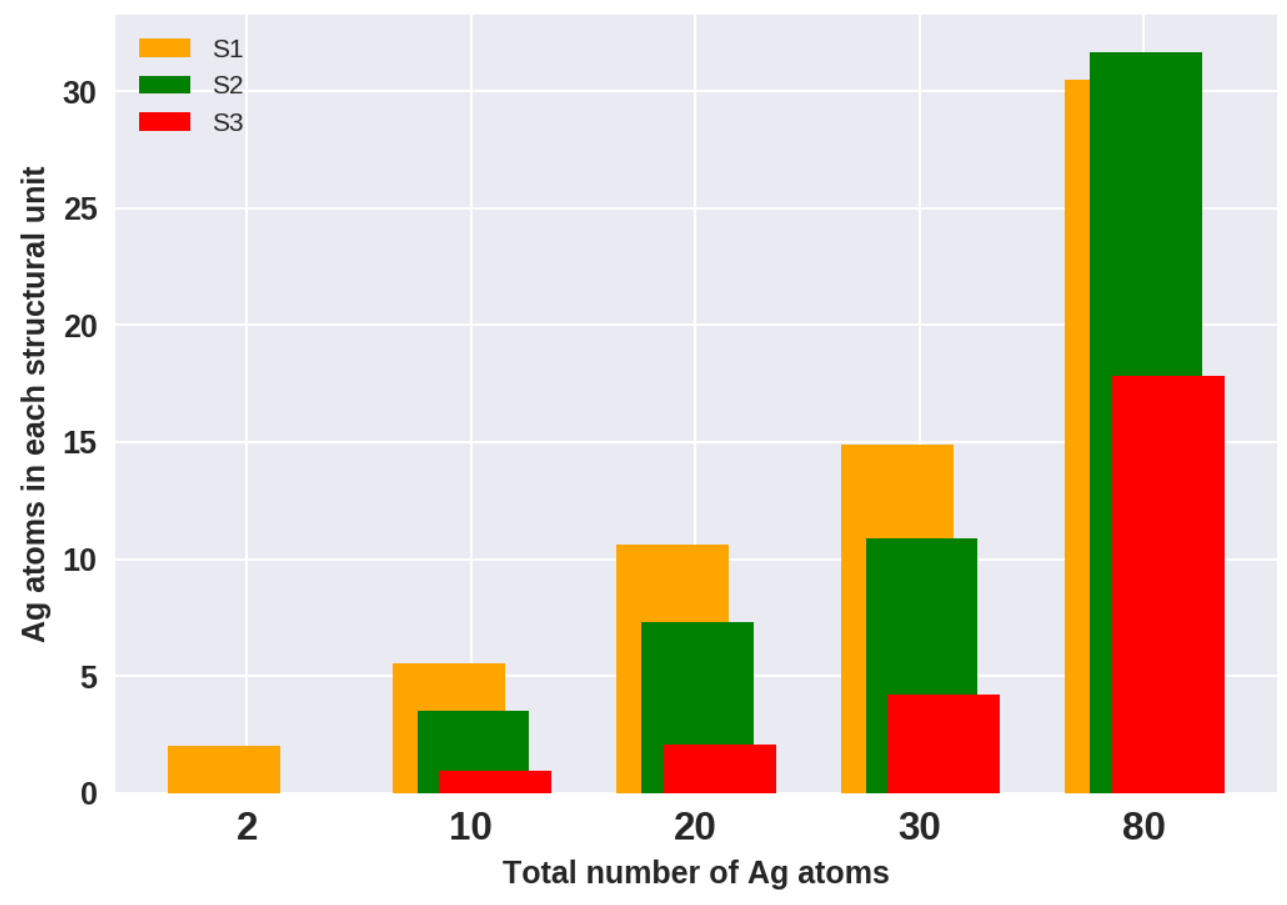

Figure 3.42: Structural trends in $\mathrm{Ag}$ alloyed $\mathrm{Au}_{102} \mathrm{SR}_{44}$

Even though slight shifts in the LUMO level can be seen for $\mathrm{x}=2$ and $\mathrm{x}$ $=10$, major changes to the electronic structure of $\mathrm{Au}_{102}$ is visible when the number of dopants increases beyond 30. With $80 \mathrm{Ag}$ atoms the HOMO-LUMO has almost disappeared. A further analysis showed that a HOMO-LUMO gap still exists in the four lowest energy structures for $\mathrm{x}=80$. However, the DOS of some of the structures having energies greater than that of the fifth lowest energy structure shows significant modulation close to the LUMO energy. The absence of a gap for $\mathrm{x}=80$ is due to these modulations being taken into account when calculating the average DOS. 


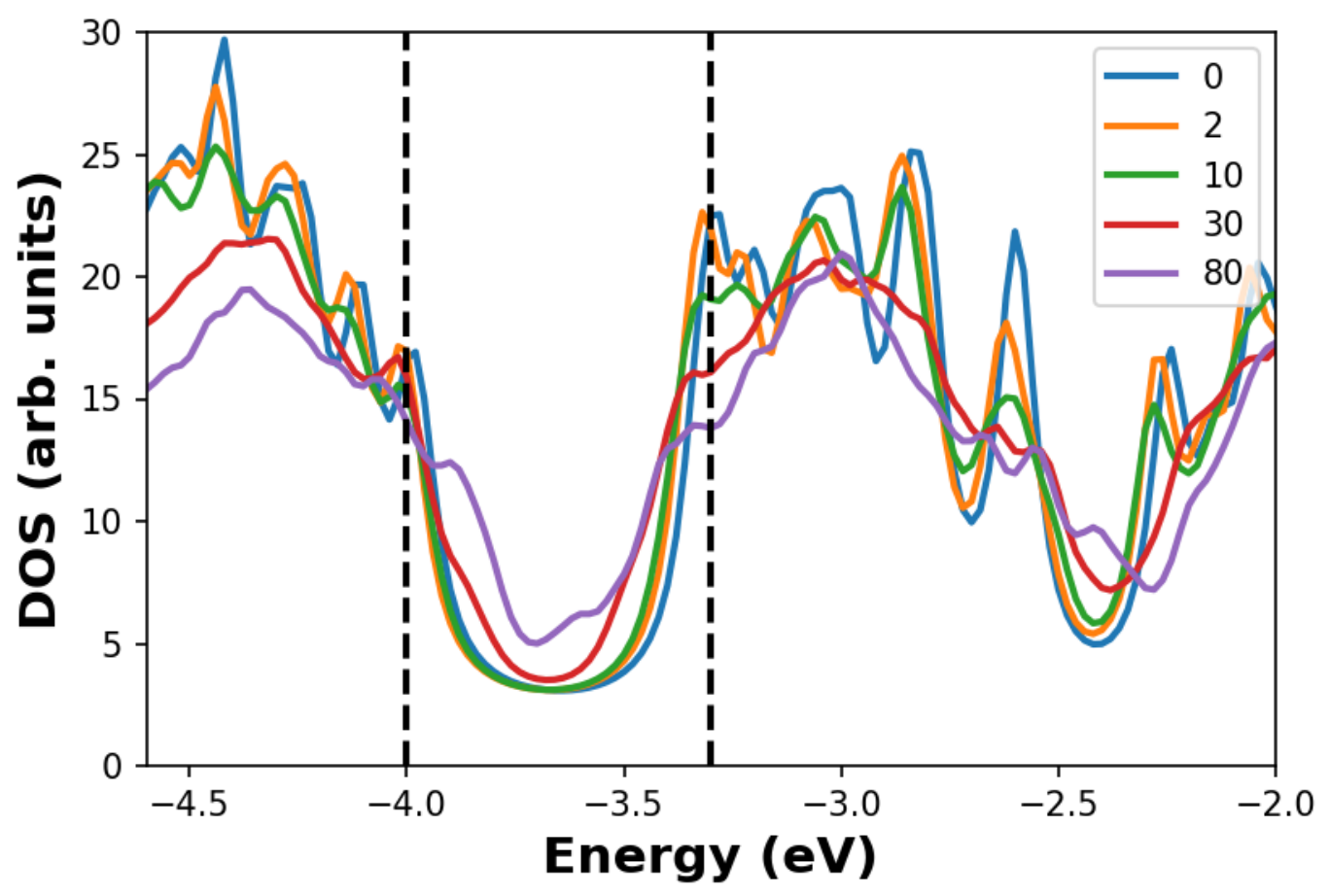

Figure 3.43: Density of states of $\mathrm{Ag}$ alloyed $\mathrm{Au}_{102} \mathrm{SR}_{44}$ 


\section{CHAPTER IV}

\section{PREDICTING THE ADSORPTION ENERGIES IN THIOLATE PROTECTED NANOCLUSTERS}

\subsection{Introduction}

Thiolated gold nanoclusters are one of the most widely studied systems in contemporary research. The increased interest isattributed to several promising applications proven for thiolated gold nanoclusters in a variety of fields including catalysis, sensing, electronics, and biomedicine. ${ }^{121,142,145,162,182-191}$ Given recent advancements in synthesis techniques, bimetallic counterparts of these systems are also produced, thereby enabling further tuning of electrochemical properties of Au-based nanoclusters and subsequently widening the scope oftheir applicability. ${ }^{33,130,137,145}$ Unfortunately, combinatorial barriers exist, which prevent fast identification of correct cluster compositions for chosen applications; subsequently, the enormity of the number of alloyed systems provides challenges for characterization. Furthermore, no theoretical methods exist to explore all combinatorially possible alloyed configurations within a practically sensible time frame. For example, the number of bimetallic configurations for the smallest known thiolated nanocluster, $\mathrm{Au}_{15}(\mathrm{SR})_{13}$, is combinatorially over 32000, which yields a significant computational challenge tocharacterize all potential 
structures. The existence of over 30 different thiolated nanoclusters composed of 15 to $144 \mathrm{Au}$ atoms increases the importance for developing smart approaches capable of making reasonable property predictions. ${ }^{192}$

In recent years, different approaches of defining coordination numbers were proposed to predict adsorption properties in metal nanoclusters without ligand passivation. ${ }^{193-197}$ How-ever, the presence of ligands containing different nonmetallic atoms oriented in different directions gives rise to extra complexity in the adsorbent-adsorbate interactions. This complexity is difficult to capture with coordination numbers alone. In this work, we propose an efficient machine-learning model aided by a fast ab initio density functional theory(DFT) approach to accomplish adsorption-energy predictions in alloyed thiolated nanoclusters. In our model, we use only the structural properties of the adsorbate-free, nonrelaxed systems. We do not use the knowledge on adsorbent-adsorbate interactions or structural/electronic properties of the optimized configurations (which requires performing many additional DFT calculations). While this can affect the prediction accuracy, our model can serve as a very fast technique to filter out a set of candidates for further testing. We demonstrate performance of the model by predicting $\mathrm{CO}$ adsorption energies in $\mathrm{Ag}$-alloyed $\mathrm{Au}_{25}(\mathrm{SR})_{18}$. $\mathrm{CO}$ oxidation has long been the preferred reaction to study the catalytic properties of Au-based systems. ${ }^{145,162,198-200}$ Also, catalytic COoxidation has been tested as a mechanism to remove poisonous $\mathrm{CO}$ from $\mathrm{H}_{2}$ in fuel cells. ${ }^{201}$ In our recent work, we found that $\mathrm{CO}$ adsorption on $\mathrm{Ag}$-alloyed $\mathrm{Au}_{25}$ is sensitive to the number of dopants, and the adsorption energies do not have a predictable trend. ${ }^{202}$ To the best of our knowledge, 
this is the first machine-learning study done for thiolated Au-based nanoclusters.

\subsubsection{Machine learning}

The definition given by Tom Michel at the Carnegie Mellon University for a machine learning algorithm is, "A computer program is said to learn from experience E with respect to some class of tasks $\mathrm{T}$ and performance measure $\mathrm{P}$ if its performance at tasks

in $\mathrm{T}$, as measured by $\mathrm{P}$, improves with experience $\mathrm{E} " .{ }^{203}$ Machine learning algorithms fall into two main categories: unsupervised and supervised learning. What is common to both these methods is the requirement to feed the machine learning algorithm with input data. This input data is a numerical representation of the domain under study and is composed one or more categorical or continuous variables - known as features in the machine-learning language. In unsupervised learning, the algorithm tries to find either trends in data or similarities among different data. Clustering is an example of unsupervised learning, where the machine learning algorithms like K-means, Gaussian mixtures and affinity propagation are tasked with grouping the input data into a particular number of groups. In supervised learning, the algorithm has to build relationships between a set of independent variables and target variable(s) based on input data. When a new set of data corresponding to independent variables are fed into the algorithm, it should be able to predict the values corresponding to the target variable(s). Based on the task at hand, supervised learning algorithms are further divided into two categories: classification and regression algorithms. 


\subsubsection{Machine learning based materials property prediction}

Several groups have successfully employed modern machine learning models to predict many electrochemical properties. In 2011, Pereira et al. used variety of models including, k nearest neighbors, random forests, support vector machines to predict Mayr electrophilicity parameter. They were able to obtain a prediction accuracy of $0.92\left(\mathrm{R}^{2}\right)$ using a support vector machine model that used descriptors. ${ }^{204}$ They also reoprted that Mayr electrophilicity is highly correlated with DFT-obtained LUMO energy. In 2012, Rupp and co-workers developed a machine learning model to predict atomization energies of molecules containing $\mathrm{C}, \mathrm{H}, \mathrm{N}, \mathrm{O}$ and $\mathrm{S} .{ }^{205}$ They defined a 'Coulomb matrix' - which is based on the chrage on each atom and the distance between any pair of atoms - to represent each molecule. Using a Gaussian kernel ridge regression model, they achived a prediction accuracy of $30.1 \mathrm{kcal} / \mathrm{mol}$ (root mean squared error(RMSE)). Also using a Gaussian kernel ridge regression model, Huan et al, predicted atomization energy, zero-point energy, isotropic polarizability, heat capacity and HOMO-LUMO gap of molecules composed of H, C, N, O, F. ${ }^{206}$ Their approach to represent a molecule was based on the number of different building blocks (for example atoms and bonds) of the molecule. An attempt to predict condensed Fukui functions using a random forest based model by Zhang et al resulted in a prediction accuracy of $0.68-0.69\left(\mathrm{R}^{2}\right) .{ }^{207}$ The Ramprasad group is known for developing machine learning models predict properties in polymers. In 2016, they Gaussian kernel ridge regression to predict band gaps and dielectric constants of polymers composed of seven different building blocks: $\mathrm{CH}_{2}, \mathrm{NH}, \mathrm{CO}, \mathrm{C}_{6} \mathrm{H}_{4}, \mathrm{C}_{4} \mathrm{H}_{2} \mathrm{~S}, \mathrm{CS}$ and $\mathrm{O} .{ }^{208}$ 
Pilania et al. also developed a kernel ridge regression model to predict band gaps in double perovskites. ${ }^{209}$ Performance of their model was evaluated using two types of features: primary and compound. Primary features consisted of Pauling's electronegativity, ionization potential, highest occupied atomic level, lowest unoccupied atomic level, and s-, p- and d- valence orbital radii. Compound features were generated by raising primary features to different powers and multiplying combinations of features. However, the model performance was found to be similar irrespective of the type of features used. Borboudakis et al employed machine learning to predict $\mathrm{CO}_{2}$ and $\mathrm{H}_{2}$ adsorption in metal organic frameworks(MOFs). ${ }^{210}$ The predictive capability of support vector regression, random forest and linear regression algorithms were recorded and the one with the best performance was selected to train the data for final prediction. Pereira et al developed a random forest based model to predict HOMO and LUMO energies of neutral organic molecules obtained with B3LYP exchange correlational functional. ${ }^{211}$ They used more than 111000 structures to train the random forest model 9989 structures to evaluate the predictive power. Mean absolute errors of 0.15 ans $0.16 \mathrm{eV}$ were achieved for HOMO and LUMO energy predictions.

The basic workflow of the models described above is as follows: (1) energetically relax the molecular/crystalline systems, (2) create a dataset containing the target property and a set of numerical fingerprints of these systems, and (3) train and test machine learning algorithms on these datasets to arrive at predictions. A major drawback of this approach is the difficulty to deal with large systems. Energetically relaxing such systems takes a long time, which compromises on the temporal 
advantages of machine learning.

\subsection{Computational Methods}

The success of any machine learning method depends on the quality of the numerical representation of the system under study. Such representations, realized as a collection of numerical fingerprints are commonly known as features. The features in our case fall into four main categories based on (i) distance between atoms, (ii) atom/bond counts, (iii) graphical representation of the cluster and (iv) volume enclosed by atoms.

We begin our investigation by examining the Au25structure, which consists of a core of $13 \mathrm{Au}$ atoms surrounded by six $\mathrm{SR}-\mathrm{Au}-\mathrm{SR}-\mathrm{Au}-\mathrm{SR}$ staple-like units, where SR stands for thiolate ligands (see Figure 3.1 in Chapter 3 for a detailed illustration of the $\mathrm{Au}_{25}$ structure). The main adsorption sites in thiolated nanoclusters are the $\mathrm{Au}$ atoms on the surface and the facets formed by surface Au atoms. ${ }^{138,142,198,212}$ Our features are applicable to any adsorption site. For the demonstration of our method, we only considered adsorption on $\mathrm{Au}$ atoms on the surface of $\mathrm{Au}_{25}$ (blue atoms in Figure 1). All the CO-adsorbed structures were energetically relaxed using the same force tolerance used for adsorbate-free $\mathrm{Au}_{25}$ structures to facilitate adsorption-energy calculation.

Distance between Ag atoms and the adsorbent site $\left(\left|\mathbf{r}_{A S-A g}\right|=\left|\mathbf{r}_{A g}-\mathbf{r}_{A S}\right|\right.$, where the abbreviation 'AS 'stands for the adsorption site) is the basis of the distance based features (see Figure $4.1(\mathrm{a})$ ). Mean and standard deviation of $\left|\mathbf{r}_{A S-A g}\right|$ form 
two basic features $(\mathrm{d} 1, \mathrm{~d} 2)$. Features $\mathrm{d} 3-\mathrm{d} 7$ are based on the centroid position of the $\mathrm{Ag}$ atoms relative to the adsorbent site. In other words, centroid is the average of $\mathbf{r}_{A S-A g}$ corresponding to all the dopant sites. Depending on whether all the Ag atoms in the cluster or just the ones in the core region are considered, two forms of centroids

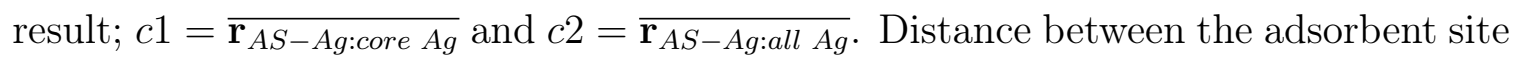
and these centroids form features $\mathrm{d} 3$ and $\mathrm{d} 4$. Features $\mathrm{d} 5$ - $\mathrm{d} 7$ measure how closely the dopant atoms are positioned with respective to their common center position.

$$
\begin{gathered}
d 5=\frac{1}{N} \sum_{\text {core } A g} \mathbf{r}_{A S-A g, i}-c 1 \\
d 6=\frac{1}{N} \sum_{\text {all } A g} \mathbf{r}_{A S-A g, i}-c 1 \\
d 7=\frac{1}{N} \sum_{\text {all } A g} \mathbf{r}_{A S-A g, i}-c 2
\end{gathered}
$$

Here,the index $i$ runs over all the $N$ number of Ag atoms in the cluster. 


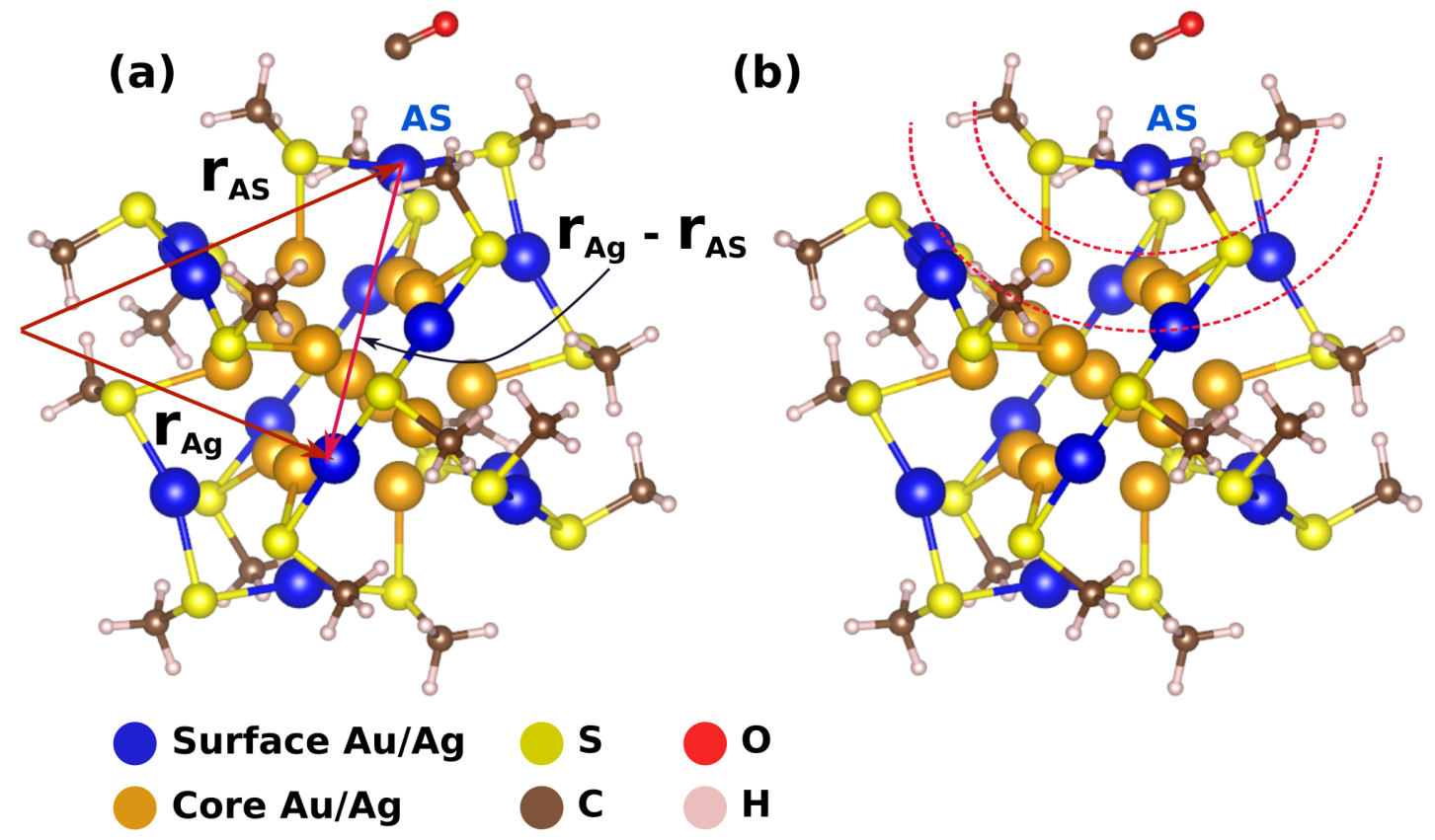

Figure 4.1: $\mathrm{CO} / \mathrm{Au} 25$ system. (a) Distance based features are based on the position of the dopant atoms relative to the adsorption site. (b) Hypothetical boundaries of the two nearest-neighbor layers are shown with dashed red curves. AS stands for the $\mathrm{CO}$ adsorbent site. Adsorbent $\mathrm{Au} / \mathrm{Ag}$ atomic sites on the surface are colored in blue. Orange is used for $\mathrm{Au} / \mathrm{Ag}$ sites in the core inaccessible for $\mathrm{CO}$ because of steric hindrance.

By identifying the atoms as belonging to different layers based on their distance to the adsorbent site, we define another class of features. As marked by the red-dashed curves in Figure 4.1 (b), boundaries of each layer are defined as concentric circles having radii equivalent to the distance between the adsorbent site and neighbors nearest to the adsorbent site. Basic building blocks of a nanocluster are 
atoms. We can also define higher order building blocks such as $\mathrm{A}-\mathrm{B}$ bonds (bonds connecting $\mathrm{A}$ and $\mathrm{B}$ atoms), or $\mathrm{A}-\mathrm{B}-\mathrm{C}$ and $\mathrm{A}-\mathrm{B}-\mathrm{C}-\mathrm{D}$ fragments in each layer "l"; accordingly, for $\mathrm{Au}_{25}$, we have created 81 atomic-bonding features. For each feature, we can subsequently make a normalized count. Fore example, if a particular layer contains six $\mathrm{C}$, three $\mathrm{H}$, and five $\mathrm{S}$ atoms,normalized counts are $6 /(6+3+5), 3 /(6$ $+3+5)$, and $5 /(6+3+5)$ for $\mathrm{C}, \mathrm{H}$, and $\mathrm{S}$, respectively.

A molecule can also be defined via graphical representation (Figure 4.2). Atoms and bonds in the molecule become nodes and edges of a graph. Using this type of representation, the following features were defined: (1) number of Ag atoms within a path length of three units from AS (g1),(2) total number of atoms within a path length of two units from $\mathrm{AS}(\mathrm{g} 2)$, (3) number of edges connecting two metal atoms within a path length of three units from AS (g3), (4) shortest path lengths between AS and the eight nearest-neighbor Au or Ag atoms (g4-g11). We add a constant to the path length when the metal atom is Ag. This gives a sequence of numbers representing the metallic environment and the relative AS-metallic atom distance. 

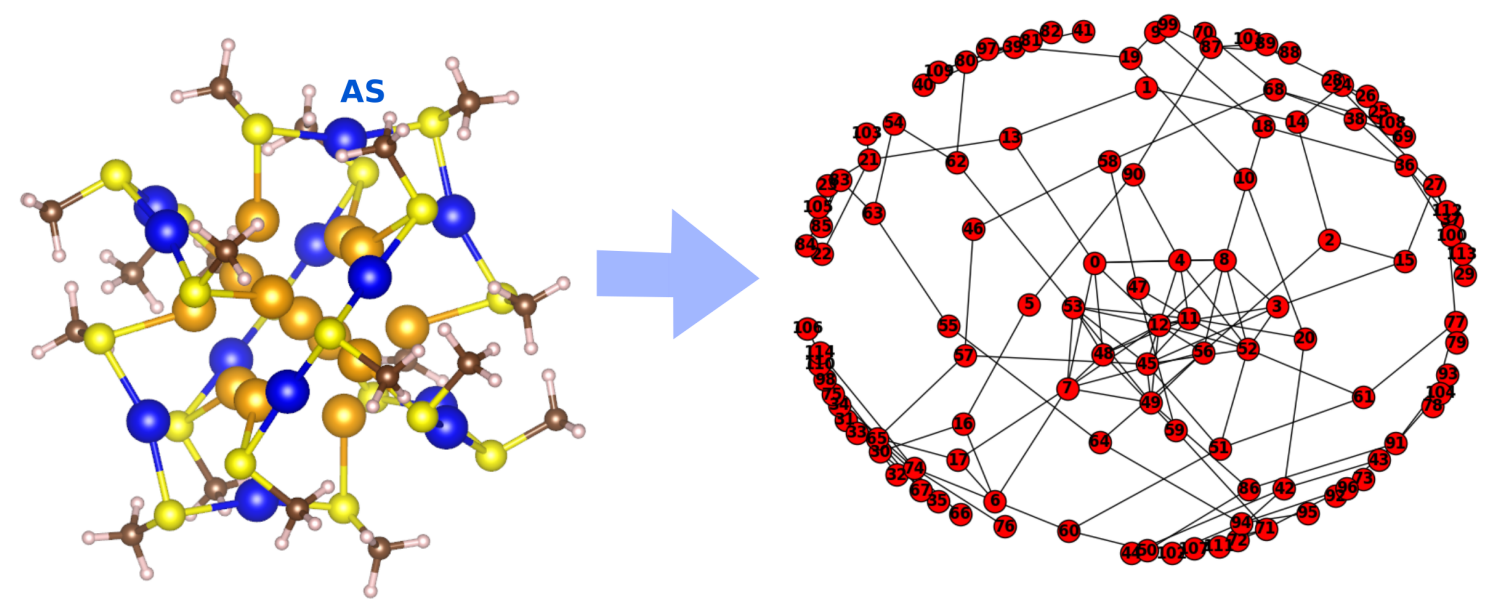

Figure 4.2: By converting the nanocluster to a graph, we defined features that provide the learning algorithm information on the connections between atoms.

Volumetric features include the volume enclosed by AS and 15 of its nearest neighbors (v1) and the volume enclosed by the AS and all the Ag sites (v2). Visual representations of these features are depicted in Figure 4.3. Apart from these features, numerous other features were also designed based specifically on the geometry of the thiolated nanoclusters. However as we wanted to create a geometry-independent model these features were not included in the final model. Nevertheless these features are discussed in APPENDIX B in the hope that a future work can make use of them after appropriate modifications. 


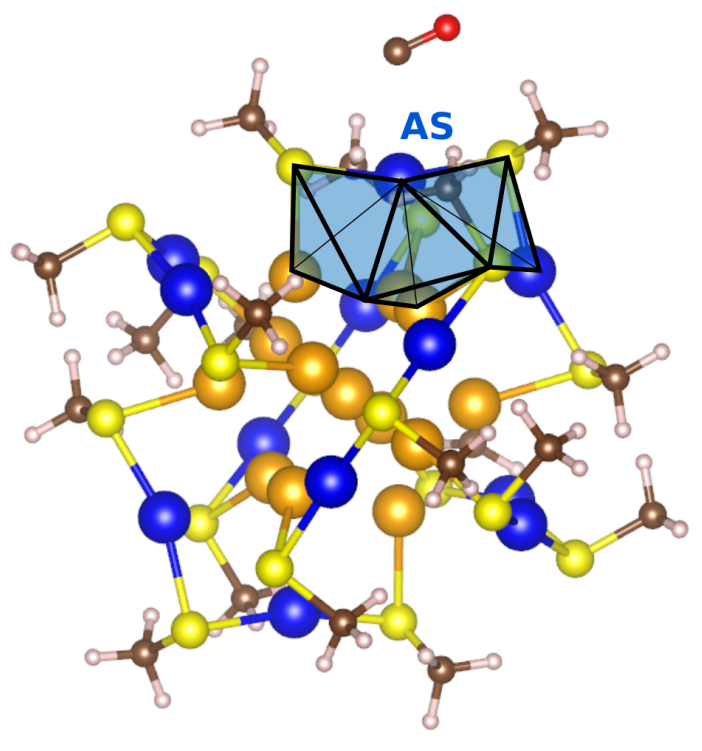

(a)

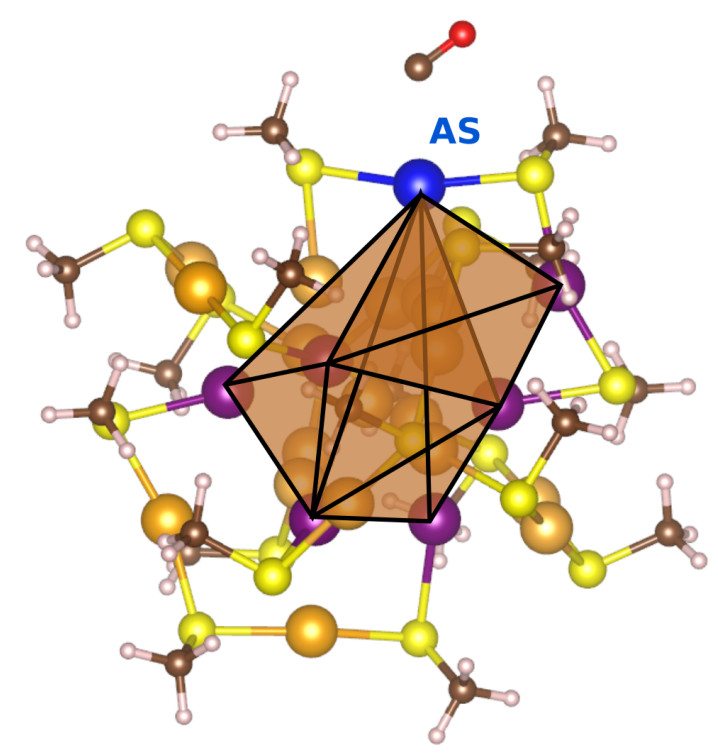

(b)

Figure 4.3: Volume based features (a) v1 and (b) v2.

Now that we have defined a set of features to describe the Au-based nanocluster and the absorbent site, we generate data from DFT calculations to discover correlations between different features and the absorbent site. Of course, machinedriven algorithms are only feasible when very large data sets are available; small data sets increase statistical anomalies. To prepare the data set, we generated $\mathrm{Ag}_{x} \mathrm{Au}_{25-x}$ nanoclusters with $x$ ranging from 1 to 25 . We created at least 500 isomers with random $\mathrm{Ag}$ sites for each alloy case, with $\mathrm{x}=4-21$. For the remaining alloying levels $(x$ $=1-3$ and $x=22-25)$, we created all possible Ag-alloyed configurations. All the calculations presented in this work were performed using FIREBALL. ${ }^{106}$ We chose the Becke ${ }^{108}$ exchange with the Lee-Yang-Parr correlation functional ${ }^{109}$ (BLYP) to 
perform structural relaxations. The basis set is made of optimized numerical local atomic orbitals, which were confined to regions limited by the corresponding cutoff radii $r_{c}$. The $r_{c}$ values used for our study are given in Appendix B. The chosen basis set and the DFT functional as implemented in FIREBALL have been successfully validated by several previous studies on Au nanoparticle systems. ${ }^{164,202,213-215}$ The adsorbate-free Ag-alloyed structures were energetically relaxed until the root-meansquare error of the force on the atoms was less than $0.05 \mathrm{eV} / \AA$.

An inspection of structural energies of $\mathrm{Ag}_{x} \mathrm{Au}_{25-x}$ revealed jumps in the energy profiles around the $15^{\text {th }}$ lowest energy for most of the alloying levels (see Figures S2 and S3 for energy profiles of alloying levels 1-24). These jumps will be hereafter referred to as "gaps "in the energy profiles. It is likely that the higher energy structures above these energy gaps have a low probability to exist. This assumption is based on the experimental studies of one-Ag-alloyed $\mathrm{Au}_{25}$, which have shown that preferable $\mathrm{Ag}$ sites are on the surface of the $\mathrm{Au}_{13}$ core. ${ }^{38,41}$ Interestingly, all the structures with a $\mathrm{Ag}$ atom on the surface of the Au13 core are the ones having energies below the gap in Figure B.2 in APPENDIX B. Therefore, a statistically meaningful data set may consist of structures with energies less than the energy corresponding to the gap. However, such a selection criteria results in poor representation from alloying levels having low-lying energy gaps. This lack of representation reduces the generalizability of the model. Hence, to maximize the number of data points, while avoiding the effect from the high-energy structures, we selected the 15 lowest energy structures from every alloying level to determine the CO adsorption-energy data. As there are 
$12 \mathrm{Au}$ sites on the surface of $\mathrm{Au}_{25}$, the number of CO-adsorbed structures considered were $12 \times 25 \times 15$.

We should note that lowest-energy adsorbate-free $\mathrm{Au}_{25}$ may not always be associated with lowest-energy CO-adsorbed structures. However, we did not restrict the number of CO-adsorbed structures based on their energies. This is because, when a reaction takes place, only the most energetically favorable $\mathrm{Au}_{25}$ have the likelihood to exist, and $\mathrm{CO}$ gets adsorbed on these stable $\mathrm{Au}_{25}$. Removing the higher energy $\mathrm{CO}-$ adsorbed data from the model results in our model being applicable only to a small niche of adsorbate-free $\mathrm{Au}_{25}$ associated with low-energy CO-adsorbed $\mathrm{Au}_{25}$. Such limitations reduce the practical importance and the generalizability of our model, as it will fail to predict the adsorption energies of most of the experimentally synthesized $\mathrm{Au}_{25}$

The adsorption energy is calculated according to $\left(E=E_{C O / A g_{x} A u_{25-x}}-\right.$ $E_{A g_{x} A u_{25-x}}-E_{C O}$ ) for CO binding at the $\mathrm{Ag} / \mathrm{Au}$ sites (at the outer shell of the nanocluster). The final data set is a (number-of-configurations) $\times$ (number-of-features +1 ) matrix, with each row corresponding to a different calculated Ag-alloyed configuration. One of the columns contains the adsorption energies, and the rest of the columns correspond to different features.

\subsection{Results and discussion}

A distribution of our calculated adsorption energies is shown in Figure ??. These energies have a mean of $-0.72 \mathrm{eV}$ and a skewness of 0.99 . The positive skewness in- 
dicates the presence of values deviating from the mean toward the positive energies. Machine-learning algorithms generally prefer normal distributions. Thus, we transformed all the adsorption energies according to $\ln (E+$ constant $)$. Here, the constant has to be chosen so that the skewness of the distribution is minimized. We found that a minimum skewness of -0.002 results when 2.5 is chosen as this constant.

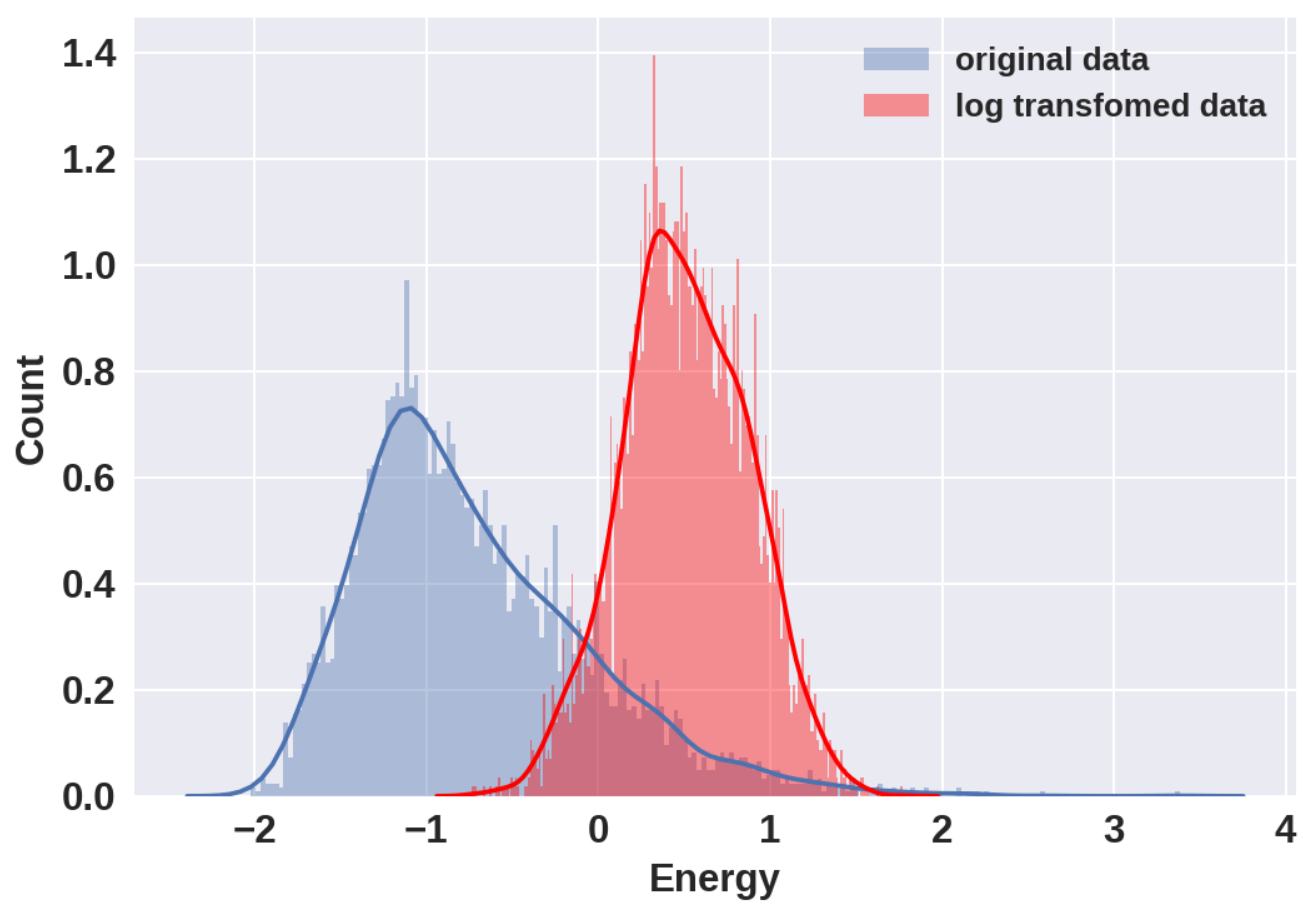

Figure 4.4: Variation of adsorption energies

Developing and testing our model consists of two phases. In the first phase, we evaluate the performance of different machine-learning algorithms using all the defined features. Tree ensemble methods like random forests 48,49 and xgboost 50 performed better than neural networks and other regression methods like linear, ridge, 
kernel ridge, and support vector regression. For this study, we chose random forests as implemented in the Scikit-learn Python library.

Random forests are a collection of decision trees. These trees are grown by choosing a particular number(n) of random samples containing a random subset of features at a time. These selections are done with replacement so that different selections may contain same samples. Final prediction is given by the average outcome of all the trees. That is,

$$
y_{\text {pred }}=\frac{1}{N} \sum_{i=1}^{N} y_{i} .
$$

More details on how a random forest is grown is given in the APPENDIX B. Next task is to train the machine learning algorithm (in our case random forests) to learn the relationships between the target variable (adsorption) energy and the various features. Our training process consists of two phases. In the firstphase, prior to feeding the features to the random-forest algorithm, each feature was scaled such that each had a zero mean and unit variance. This is to ensure that no single feature will dominate the objective function of the algorithm, ignoring the effects of other features with lower variance. Next, we randomly split the whole data set as training and testing sets according to an 80:20 ratio. Using the data in the training set, a random forest with 100 trees was trained with five-fold cross-validation (explained in APPENDIX B). We used the built-in feature ranking method in random forests to determine the best features based on their contribution to the prediction accuracy. Figure 4.5 shows the top 10 features along with their Pearson correlation coefficient 
and mutual information values with respect to the adsorption energy. The Pearson correlation coefficient quantifies the linear dependence between two variables, while mutual information is a measure of both linear and nonlinear dependence. The top 10 features have decent linear dependencies with adsorption energy, even though our features are based on the geometric properties of nonrelaxed structures. As the adsorption energies were calculated using DFT-relaxed structures, these correlation values indicate that our features are likely related to the local chemical environment at the vicinity of adsorbent sites of the synthesized structures. Not surprisingly, the simplest possible feature for an alloyed system, the number of dopant Ag atoms (nAg) has the greatest effect on the prediction accuracy. However, as will be shown, nAg alone is not enough to achieve the maximum accuracy. It is also worth noting that all the distance-based features are among the top 10 features. 


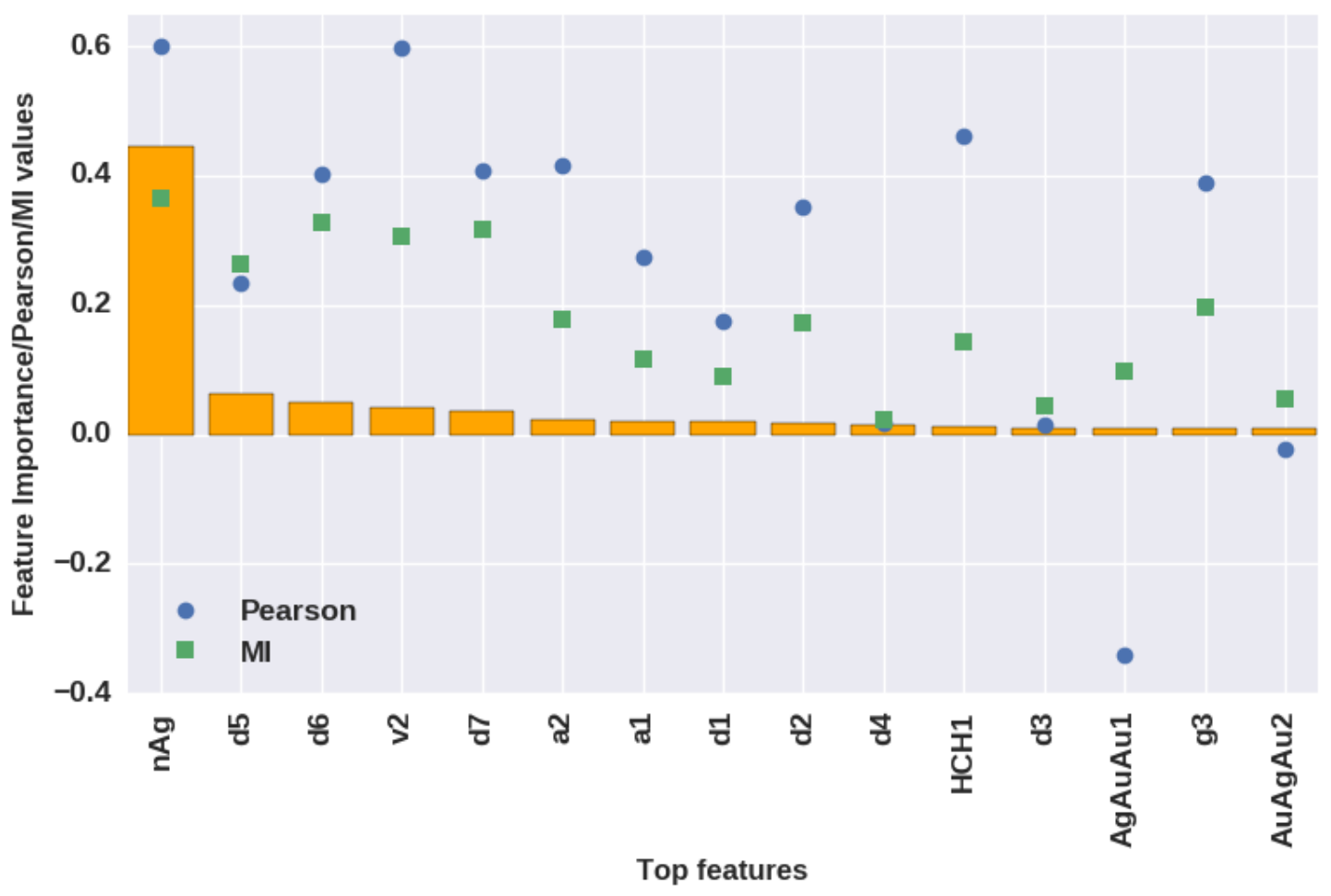

Figure 4.5: Highest ranking important features selected by random forests and the corresponding Pearson correlation coefficient and mutual information values. $\mathrm{nAg}$ is the number of $\mathrm{Ag}$ atoms; $\mathrm{d} 3-\mathrm{d} 5$ are measures of how closely the $\mathrm{Ag}$ atoms are clustered around the centroid of the Ag locations relative to the adsorbent site; $\mathrm{HCH} 1$ is the normalized number of $\mathrm{H}-\mathrm{C}-\mathrm{H}$ fragments in the first layer of neighbors nearest to the adsorbent site; $\mathrm{d} 1$ and $\mathrm{d} 2$ are the mean and standard deviation of $\left|\mathrm{r}_{A S-A g}\right|$, and $\mathrm{v} 2$ is the volume enclosed by the adsorbent site and the Ag sites.

The feature-feature correlations in Figure 4.6 indicate that the number of dopant $\mathrm{Ag}$ atoms has the greatest correlation to the other top 14 features (the feature 
v2 has the next highest correlation to the other features). One interesting aspect is that Ag dependency is directly inherent in many of the defined features, particularly features based on centroids and bonding information. However, the number of dopant $\mathrm{Ag}$ atoms are not directly defined within the feature $\mathrm{HCH} 1$, which corresponds to the normalized number of $\mathrm{H}-\mathrm{C}-\mathrm{H}$ fragments in the first layer of neighbors nearest to the adsorbent site, as it is independent of the number of dopant Ag atoms. Surprisingly, despite the lack of defined dependency between the two features nAg and $\mathrm{HCH} 1$, there is a strong correlation between these two features (value of 0.68) as indicated in the correlation map of Figure 4.6. 


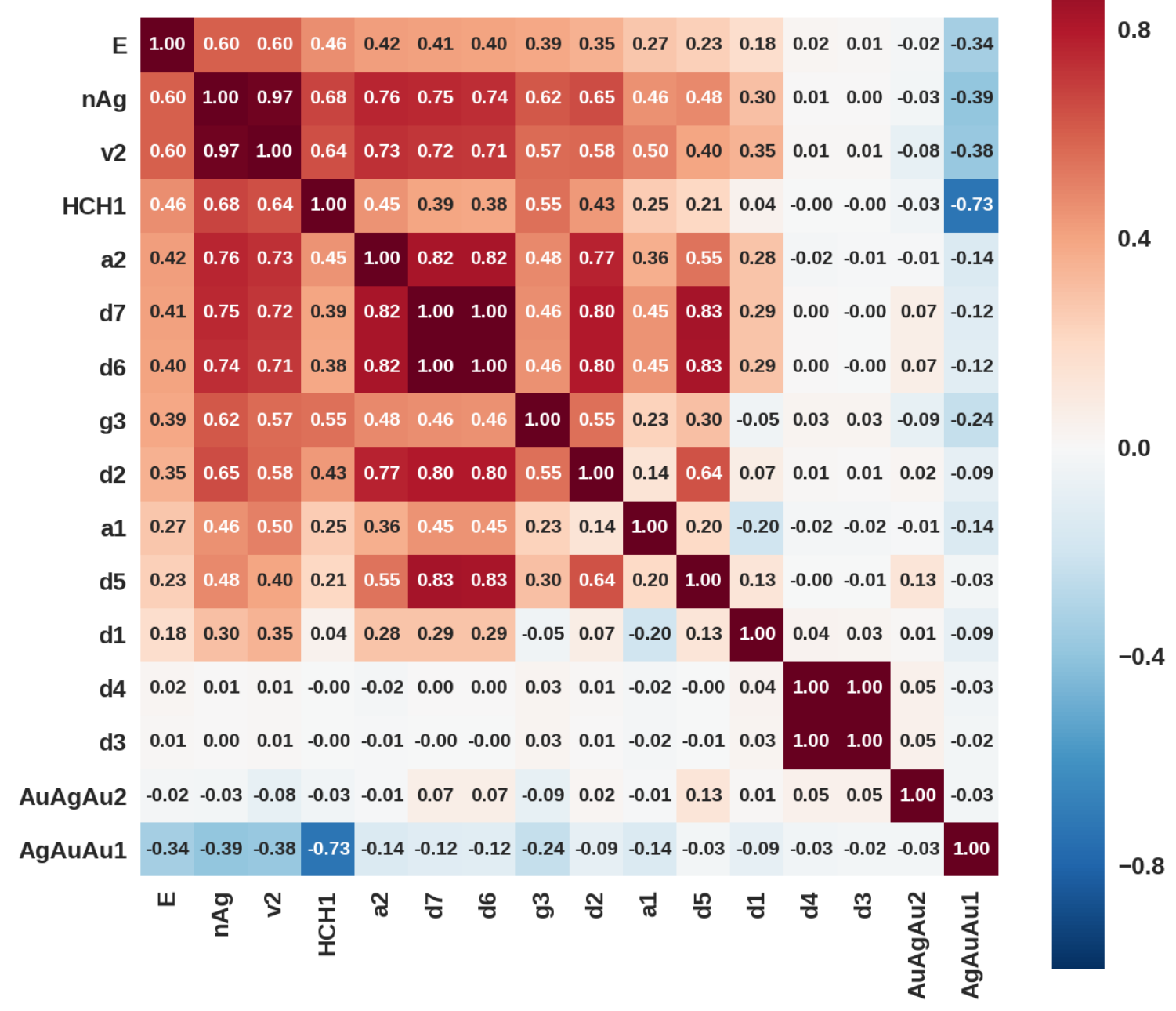

Figure 4.6: Features that are highly correlated with the adsorption energy according to Pearson correlation measure.

The features specifically designed to measure the clustering of the Ag (d5, $\mathrm{d} 6$, and $\mathrm{d} 7$ ) are among the top five features. It is surprising to find that $\mathrm{d} 6$ and d7 contain almost the same information on linear dependence (correlation coefficient of 0.99), even though they are based on different centroids. This strong correlation 
indicates that Ag atoms are clustered around a similar central position. Despite the close relationship between $\mathrm{d} 6$ and $\mathrm{d} 7$, random forests has considered both as highly important. Even though d5 has the lowest correlation values compared with the top 5 , it has been ranked as the second most important feature. This could be due to d5 having interactions with the other features. Even v2 is an indirect measure of the clustering, as the volume enclosed by the adsorbent site and the $\mathrm{Ag}$ atoms decreases with Ag atoms being clustered within a proximity to each other.

The counts of building blocks were previously shown to be effective in other systems, and we were inspired to add these as features for our investigation,?,?,206,207 however, we note relatively poor scores for building block features with one exception. The occurrence of $\mathrm{HCH} 1$ (number of $\mathrm{HCH}$ units in layer 1) as one of the important features (seen in Figure 4.5) shows that orientation of ligands also plays a significant role in predicting the adsorption energies. This is because $\mathrm{H}-\mathrm{C}-\mathrm{H}$ units are only found in the ligands that are more readily located near CO adsorption sites.

There is no strong agreement between the trends in Pearson correlation and mutual information with that of the feature importance, even though for features between $\mathrm{nAg}$ and d1 in Figure 3, high mutual information values are generally associated with high feature importance. This was further confirmed by constructing an extended correlation map by considering more features (for example, see Figure B.4 in APPENDIX C). We notice that the features $\mathrm{d} 5-\mathrm{d} 7$ and $\mathrm{v} 2$ are among the highest scoring mutual information values but not among the features with highest Pearson correlation coefficients. This may indicate that nonlinear relationships between the 
adsorption energy and the features play an important role in the prediction. We also note that the features having a negative linear correlation with adsorption energy (for example, AgAuAu1 and AuAgAu2) have low effect on the prediction.

Once the feature importances are found using the random forest model, we use another random-forest model (also with 100 trees) to find the best subset of features that maximizes the prediction accuracy. For example, as shown in Table 4.1 we iteratively increase the number of top features (as determined by the first random forest) included in the second random forest and find the features corresponding to the best accuracy.

\begin{tabular}{|l|c|l|}
\hline Number of top features & $\mathrm{R}^{2}$ (validation) & Features included \\
\hline 1 & 0.483 & $\mathrm{nAg}$ \\
\hline 2 & 0.746 & $\mathrm{nAg}, \mathrm{d} 5$, \\
\hline 3 & 0.765 & $\mathrm{nAg}, \mathrm{d} 5, \mathrm{~d} 6$, \\
\hline 4 & 0.726 & $\mathrm{nAg}, \mathrm{d} 5, \mathrm{~d} 6, \mathrm{v} 2$, \\
\hline 5 & 0.728 & $\mathrm{nAg}, \mathrm{d} 5, \mathrm{v} 2, \mathrm{~d} 6, \mathrm{~d} 7$ \\
\hline 6 & 0.706 & $\mathrm{nAg}, \mathrm{d} 5, \mathrm{~d} 6, \mathrm{v} 2, \mathrm{~d} 7, \mathrm{a} 2$ \\
\hline
\end{tabular}

Table 4.1: Change in the validation accuracy $\left(\mathrm{R}^{2}\right)$ with respect to the number of best features in phase 1.

Using the new set of features selected in the cross-validation (highlighted in 
Table 4.1 ), we can create additional features in the second phase known as feature engineering. Accordingly, new features were created as $x^{2}, \mathrm{x}^{3}, \sqrt{x}$ and $\ln (x)$ for $x$ $=\mathrm{nAg}, \mathrm{d} 5$ and $\mathrm{d} 6$. As a result, the dataset used for the second phase of training contains nAg, d5 and d6 and the newly engineered features. Similar to phase 1, the optimal features were selected inside a 5 -fold cross validation loop. Table 4.2 shows the change in the validation accuracy with the number of top features $n$, for $n=1$ to 9 .

\begin{tabular}{|c|c|c|}
\hline Number of top features & R2 (validation) & Features included \\
\hline 1 & 0.48296 & $\mathrm{nAg}$ \\
\hline 2 & 0.48296 & $\mathrm{nAg}, \mathrm{nAg}^{2}$ \\
\hline 3 & 0.48296 & $\mathrm{nAg}^{2}, \mathrm{nAg}, \mathrm{nAg}^{3}$ \\
\hline 4 & 0.77029 & $\mathrm{nAg}, \mathrm{nAg}^{2}, \mathrm{nAg}^{3}, \mathrm{~d} 6$ \\
\hline 5 & 0.77037 & $\mathrm{nAg}, \mathrm{nAg}^{2}, \mathrm{nAg}^{3}, \mathrm{~d} 6, \mathrm{~d} 6^{3}$ \\
\hline 6 & 0.77028 & $\mathrm{nAg}, \mathrm{nAg}^{2}, \mathrm{nAg} \mathrm{g}^{3}, \mathrm{~d} 6, \mathrm{~d} 6^{3}, \mathrm{~d} 6^{2}$ \\
\hline 7 & 0.77017 & $\mathrm{nAg}, \mathrm{nAg}^{2}, \mathrm{nAg} \mathrm{g}^{3}, \mathrm{~d} 6, \mathrm{~d} 6^{3}, \mathrm{~d} 6^{2}, \mathrm{~d} 5^{3}$ \\
\hline 8 & 0.77017 & $\mathrm{nAg}, \mathrm{nAg} g^{2}, \mathrm{nAg} g^{3}, \mathrm{~d} 6, \mathrm{~d} 6^{3}, \mathrm{~d} 6^{2}, \mathrm{~d} 5^{2}, \mathrm{~d} 5^{3}$ \\
\hline 9 & 0.77015 & $\mathrm{nAg}, \mathrm{nAg}{ }^{2}, \mathrm{nAg} g^{3}, \mathrm{~d} 6, \mathrm{~d} 6^{3}, \mathrm{~d} 6^{2}, \mathrm{~d} 5^{3}, \mathrm{~d} 5, \mathrm{~d} 5^{2}$ \\
\hline
\end{tabular}

Table 4.2: Change in the validation accuracy $\left(\mathrm{R}^{2}\right)$ with respect to the number of best features in our second phase of training. 
Creating more complicated features, like adding and multiplying features in two or three feature combinations and calculating the Euclidian distance between feature values and their cluster centers, did not result in improvement of the prediction accuracy. Following the same approach as the first phase, the best features were filtered out from the ones selected in the first phase and those engineered based on them. Additionally, parameter tuning of the random-forest method was also performed to determine the optimal parameters of the final model. However, no significant gain in the accuracy was obtained with different parameters. Therefore, the final model contains only 100 trees with default parameters.

The features in the final model are the ones of the best cross-validation step. These are $\mathrm{nAg}, \mathrm{nAg}^{2}, \mathrm{nAg}^{3}, \mathrm{~d} 6$, and $\mathrm{d} 6^{3}$ (highlighted ib Table 4.2). This result shows that adsorption energies in $\mathrm{Au}_{25}$ can be predicted with only two features along with their nonlinear counterparts. This model was then trained once with the whole training set and tested with the testing set to arrive at the final prediction accuracy.

The accuracy of our model prediction is shown in the prediction performance plot (log transformed), Figure 4.7 (a) with the distribution of residual error shown in Figure 4.7. Prediction accuracies of the log transformed data are, $\mathrm{R}^{2}=0.77869$, $\mathrm{MAE}=0.13196$, and RMSE $=0.17348$. Residual errors (predicted-actual) of the prediction are shown in Figure ??. The higher the residual error, the more erroneous the result. For negative and positive adsorption energies, the mean of the absolute residual errors are 0.20 and $0.44 \mathrm{eV}$, respectively. Predicted values corresponding to positive adsorption energies have more deviation from the actual values compared to 
the negative adsorption energies (see Figure B.5 in APPENDIX C). Positive adsorption energies correspond to weak $\mathrm{CO} / \mathrm{Au}_{25}$ attraction. The potential energy surface consists of many local minima with similar energies, which results in structurally different CO-adsorbed isomers having similar adsorption energies. Shallow potential energy surfaces do make it difficult for the learning algorithm to accurately predict positive adsorption energies. Another major factor affecting the accuracy of our model may be that the ligands are oriented in different directions. Ligands create a complex chemical environment for adsorbate molecules. As shown in Figures 4.5 and 4.6, adsorption energies are mainly affected by the location of Ag atoms and the $\mathrm{H}-\mathrm{C}-\mathrm{H}$ fragments closest to the adsorption site. To capture the combined effect of ligands and dopants, we fed our model with compound features created by multiplying features. However, these new features did not result in improvements for the model accuracy. Further, the use of nonrelaxed isomers to generate features is also detrimental to the prediction accuracy. This is because the presence of dopants significantly affects bonding, and atom-atom distances of a relaxed structure may be highly deviated from those of the nonrelaxed structure. Our model was unable to learn without ambiguity; however, there is a definite relationship between the positive adsorption energies and structural parameters. We also tried to build two models for positive and negative adsorption energies. However, this did not result in an increased accuracy, which could be due to the reduced number of data points in each sample. 


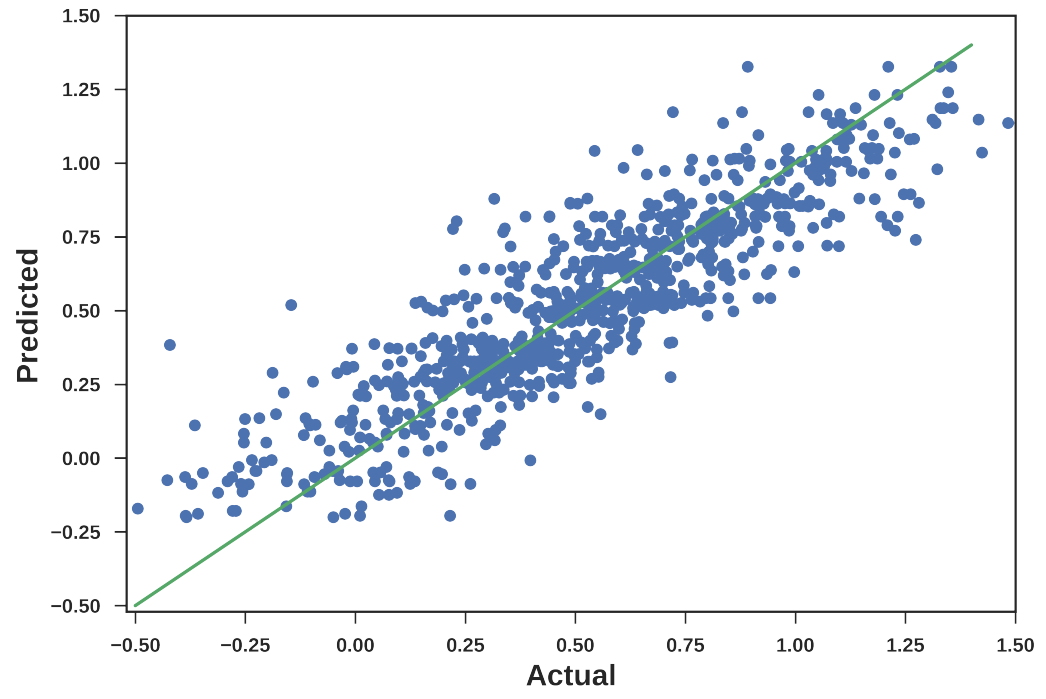

(a)

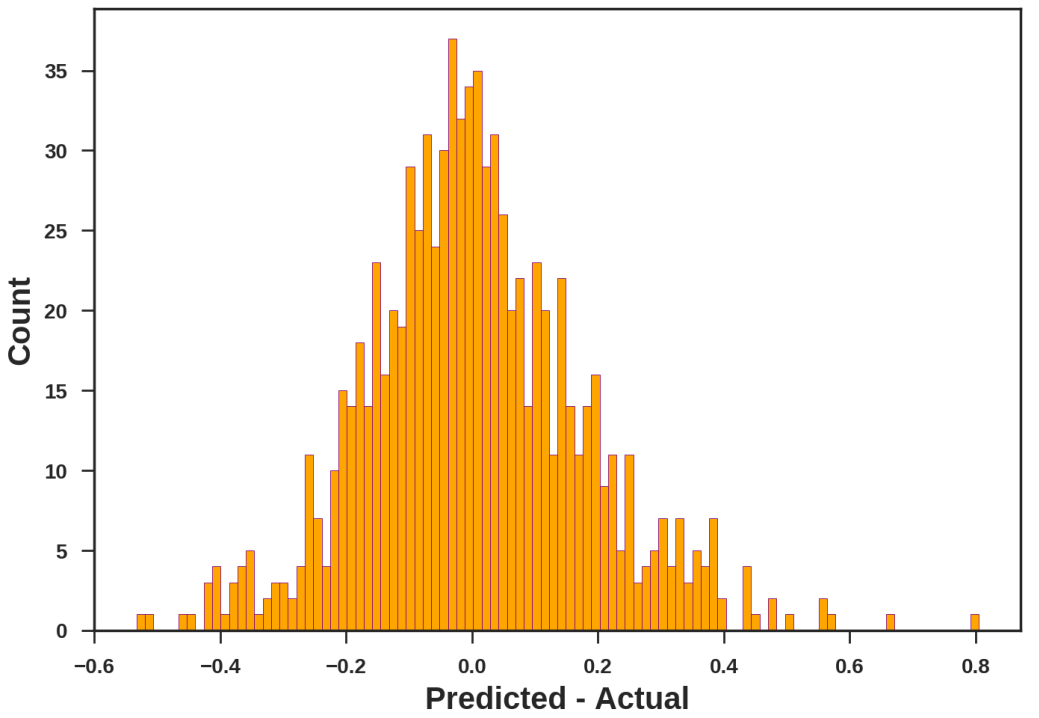

(b)

Figure 4.7: Prediction performance plots of the final model for $\mathrm{Au}_{25}$ nanocluster: (a) actual vs predicted adsorption energies and (b) histogram counts of (predicted-actual) adsorption energies. 
Few points about the our feature selection process need to be clarified. This procedure assumes that the order of feature importance remains the same as the size of the subset of features which is considered to evaluate the model accuracy gets smaller. To check whether this assumption is true, we replaced $\mathrm{d} 5$ with other top features and obtained the corresponding prediction accuracies as listed in Table 4.3. It is interesting that (nAg, d6) and (nAg, d7) combinations result in higher accuracies than the $(\mathrm{nAg}, \mathrm{d} 5)$ combination. This shows that the order of the feature importance is not necessarily preserved when the size of the feature space reduces. However, we also see from Table 4.3 that features which are not highly correlated with d5 cannot be used to replace d5 to obtain accuracies similar to (nAg, d5) combination. What we can do to rectify this issue is to remove features one by one and re-calculate feature importances after each removal. This procedure significantly increases the feature selection time and is not feasible when the number of features is at least over 20. However, we notice that in the second phase of feature selection, d5 has been discarded in favor of d6-based features which shows that our two-phase feature selection procedure can be considered as a time efficient alternative to the above mentioned feature-importance re-calculation step. We also notice that the maximum prediction accuracy of phase two is equal to that obtained with (nAg,d6) combination in phase 1 . This raises the question whether we actually need to perform phase two? The answer to this question lies in the philosophy behind feature engineering, which is to create features containing new information about the domain being featurized. One of the reasons for raising original features to different powers is to create features that 
may have non-linear relationships with the predicted variable (in this case adsorption energies). Also this kind of features can be used to create aggregated features of the form $x^{a} y^{b} z^{c}$, where $x, y$ and $z$ belong to the original feature space and $a, b$, and $c$ are some arbitrary numbers. We understand that this procedure may not result in physically meaningful features and may contain similar information as that of the original features. However, our results show that our current procedure has been able to achieve maximum possible accuracy without the use of advanced feature-filtering steps. Nevertheless, in a future work, we hope to implement Pearson correlation and mutual information based feature selection procedures to make sure that the resulting increase in the training time can be compensated for a gain in the prediction accuracy.

\begin{tabular}{|c|l|l|}
\hline $\mathrm{R}^{2}$ (validation) & Features considered $(\mathrm{nAg}, \mathrm{x})$ & Pearson correlation between $\mathrm{nAg}$ and $\mathrm{x}$ \\
\hline 0.770 & $\mathrm{nAg}, \mathrm{d} 6$ & 0.83 \\
\hline 0.770 & $\mathrm{nAg}, \mathrm{d} 7$ & 0.83 \\
\hline 0.746 & $\mathrm{nAg}, \mathrm{d} 5$ & 0.48 \\
\hline 0.479 & $\mathrm{nAg}, \mathrm{v} 2$ & 0.40 \\
\hline 0.340 & $\mathrm{nAg}, \mathrm{a} 2$ & 0.55 \\
\hline 0.337 & $\mathrm{nAg}, \mathrm{d} 1$ & 0.64 \\
\hline
\end{tabular}

Table 4.3: Effect of replacing d5 with other top features on the prediction accuracy when only two features are included in the random forest model. 
We extend our model to $\mathrm{Au}_{36}(\mathrm{SR})_{24}$ and $\mathrm{Au}_{133}(\mathrm{SR})_{52}$ nanoclusters, thereby demonstrating the versatility of our model. For $\mathrm{Au}_{36}(\mathrm{SR})_{24}$, we used only 1360 isomers having either $1,2,3,6,9,12,13,18$, or $24 \mathrm{Ag}$ atoms. At the second phase of feature selection, the best features were found to be $d 7, d 7^{3}, H C 1^{2}, d 7^{2}, H C 1$, $H C 1^{3}$, which gave accuracies of $0.65388\left(\mathrm{R}^{2}\right), 0.21582$ (RMSE), and 0.1856 (MAE) for predictions (prediction performance plot shown in Figure 4.8 (a)). 


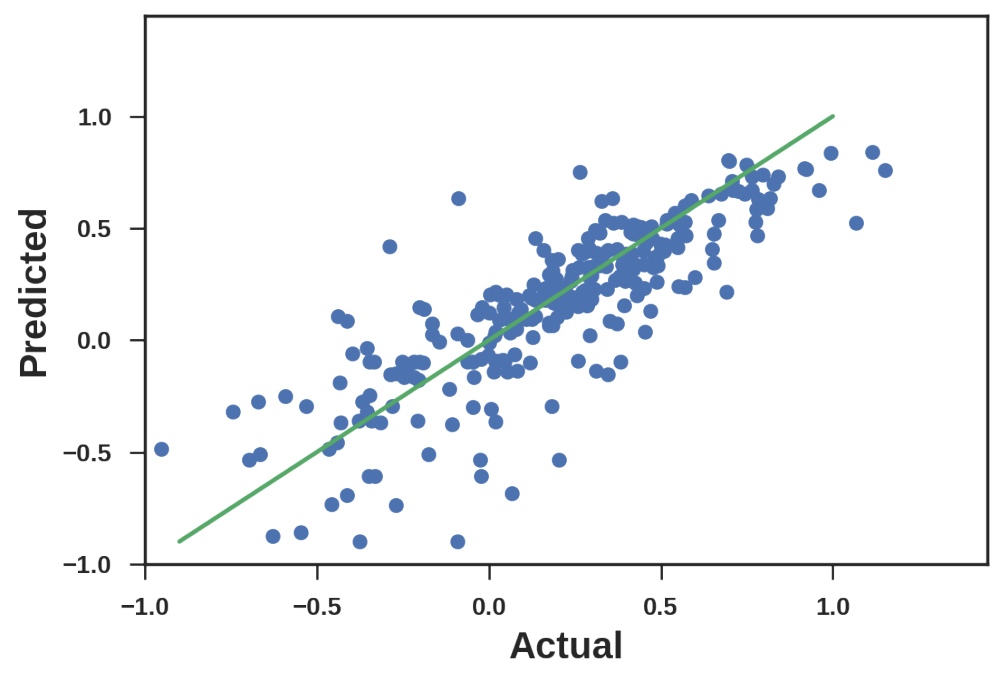

(a)

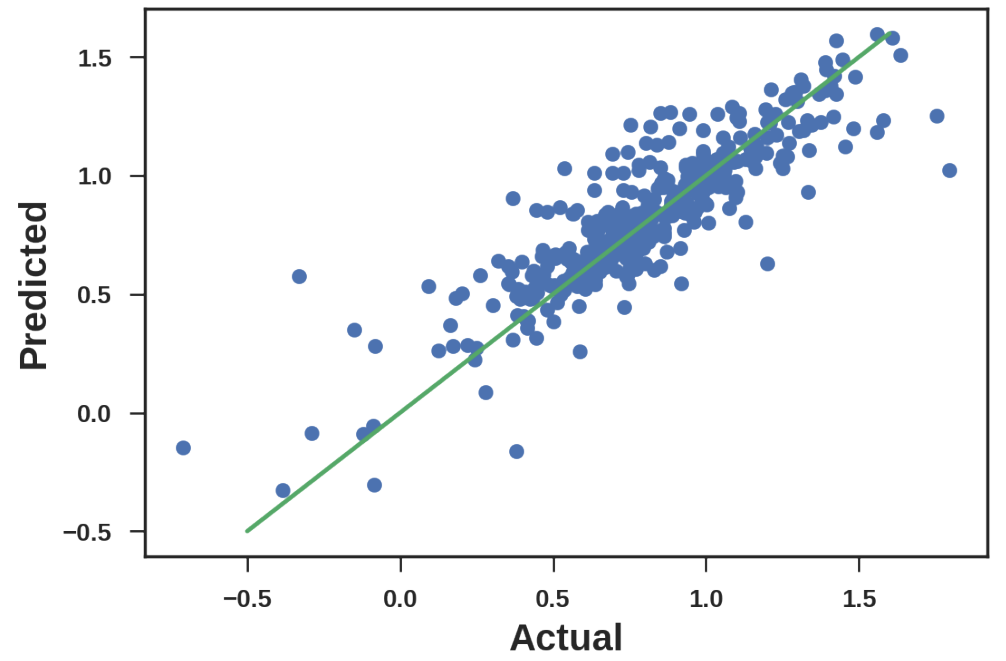

(b)

Figure 4.8: Prediction performance plots for (a) $\mathrm{Au}_{36}$ and (b) $\mathrm{Au}_{133}$ nanoclusters. 
These accuracies are encouraging, given the less symmetric geometry of $\mathrm{Au}_{36}$ in comparison to $\mathrm{Au}_{25}$ and the smaller sample size used for the training. $\mathrm{As}^{\mathrm{Au}} \mathrm{u}_{36}$ is relatively a small cluster, the number of samples can be increased easily to achieve a possible increase in the prediction accuracy. These accuracies also show that the features defined based on the nearly spherical $\mathrm{Au}_{25}$ cluster can be readily used for the clusters like $\mathrm{Au}_{36}$, which have different symmetries. The $\mathrm{Au}_{133}(\mathrm{SR})_{52}$ nanocluster is the largest thiolated nanocluster, of which the crystal structure has been verified. This nanocluster contains 393 atoms with $-\mathrm{SCH}_{3}$ as the ligand. We generated 1898 CO-adsorbed isomers having 5, 10, 15, 20, 30, 48, 80, and $120 \mathrm{Ag}$ atoms. Even with a small number of samples, we were able to achieve accuracies of $0.75063\left(\mathrm{R}^{2}\right), 0.17128$ (RMSE), and 0.11882 (MAE) for predictions (prediction performance plot shown in Figure 4.8 (b)). This shows that our model may be well suited for spherical-like clusters. The best features selected at the second phase of feature selection were $n A g^{3}, n A g, n A g^{2}, d 5, d 5^{2}, d 5^{3}, d 6^{3}, d 6^{2}, d 6, d 7^{3}, d 7, d 7^{2}, v 2, g 3^{3}, g 3^{2}, g 3, d 1^{2}, g 2^{2}$, $v 2^{2}, d 2^{2}$.

Interestingly, in $\mathrm{Au}_{36}$, features based on the orientation of the ligands have a significant effect on the prediction accuracy, whereas in $\mathrm{Au}_{25}$ and $\mathrm{Au}_{133}$, only the dopant-based features have the strongest influence. We hypothesize that this is due to the ligands in $\mathrm{Au}_{36}$ causing a higher steric hindrance than the ligands in either $\mathrm{Au}_{25}$ or $\mathrm{Au}_{133}$. To test this hypothesis, we can use the feature $\mathrm{HC} 1$, the normalized count of $\mathrm{H}-\mathrm{C}$ bonds within the 10 nearest neighbors of the adsorbent sites. The higher the number of $\mathrm{H}-\mathrm{C}$ bonds close to the adsorbent site, the larger is the steric hindrance. 
For each adsorbate-free nanocluster, we calculated the average of $\mathrm{HC} 1$ over all the adsorbent sites. The averages obtained for $\mathrm{Au}_{25}, \mathrm{Au}_{36}$, and $\mathrm{Au}_{133}$ are 0.10, 0.14, and 0.09 respectively. Thus, there are more $\mathrm{H}-\mathrm{C}$ bonds closer to the adsorbent sites in $\mathrm{Au}_{36}$ compared to other two nanoclusters, resulting in greater steric hindrance to approaching $\mathrm{CO}$ adsorbates. The steric hindrance from $\mathrm{H}-\mathrm{C}$ bonds is likely related to the sphericity ${ }^{216}$ of the core of the nanoclusters. The closer the value of sphericity to 1 , the more spherical is the cluster. More spherical surfaces tend to cause less steric hindrance as illustrated in Figure 4.9. As shown in Figure B.7 in APPENDIX B, the core of Au36 consists of planar-like surfaces with a sphericity of 0.735 . Sphericities of $\mathrm{Au}_{25}$ and $\mathrm{Au}_{133}$ cores are 0.940 and 0.943 respectively.
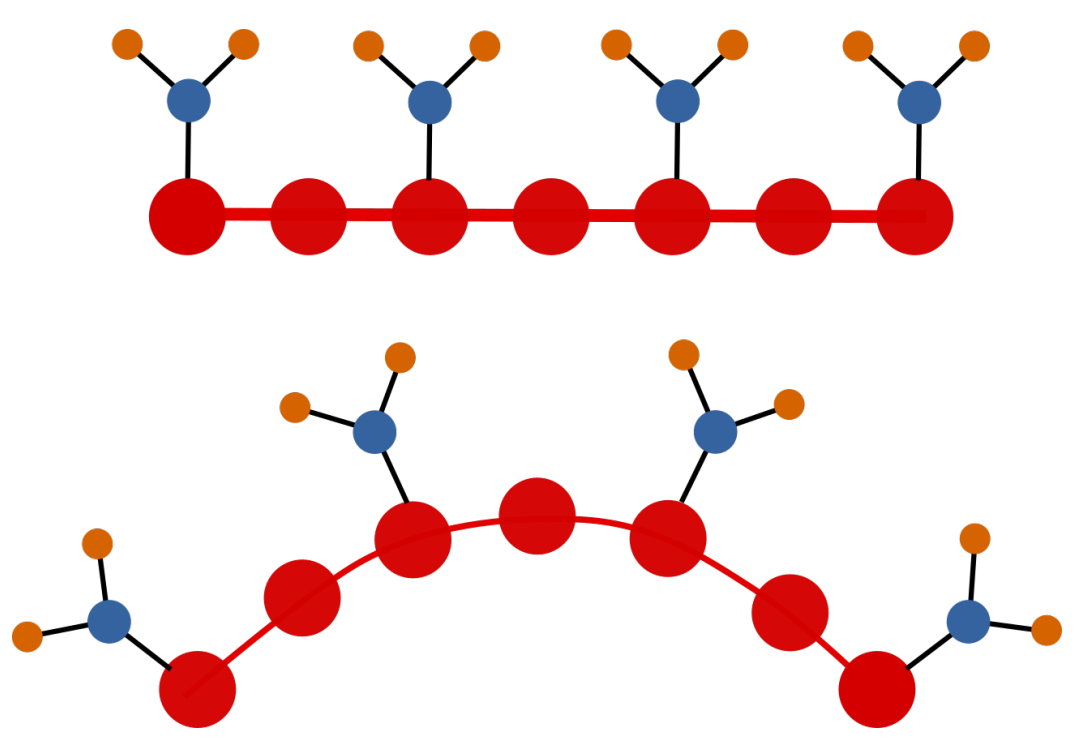

Figure 4.9: The steric hindrance from the ligands on a spherical surface (bottom) is more than the steric hindrance from the ligands on a more planar surface (top). Color code: surface atoms, red; ligands atoms, blue, orange. 


\subsection{Conclusions}

Overall, we have developed a machine-learning model based on the random-forest method to predict $\mathrm{CO}$ adsorption energies for Au-based nanoclusters, starting by training our model with the $\mathrm{Au}_{25}$ nanocluster alloyed with $\mathrm{Ag}$. We have defined approximately 100 features to model nanoclusters as numerical representations. Over 2000 data points are contained in our model. Using a two-step feature-selection process, and feature engineering approaches, we predicted the adsorption energies of $\mathrm{Au}_{25}$ with accuracies of $0.78\left(\mathrm{R}^{2}\right)$ and 0.17 (RMSE). Our chosen features are based merely on the structural properties of the unoptimized adsorbate-free system (to enable rapid prediction); our model is an excellent filtering tool to select first round candidates for further, more accurate, analysis. The validity of our model was also tested by predicting $\mathrm{CO}$ adsorption energies in the less symmetric $\mathrm{Au}_{36}$ nanocluster and the larger $\mathrm{Au}_{133}$ nanocluster, using the same defined features as the $\mathrm{Au}_{25}$ model, with prediction accuracies $\left(\mathrm{R}^{2}\right)$ of 0.65 and 0.75 , respectively. 
CHAPTER V

STRUCTURAL AND ELECTRONIC PROPERTIES OF FE ALLOYED AG DELAFOSSITES

\subsection{Introduction}

Due to having wide optical band gaps, ${ }^{63,71,79,81,82,217-219} \mathrm{n}^{52,57}$ and p-type $\mathrm{e}^{54,56,62-64,217}$ conductivities, delafossites are widely known as transparent conducting oxides for photovoltaic applications. They have also been tested for other applications in catalysis, ${ }^{66,68,72,220-222}$ batteries, ${ }^{223,224}$ thermoelectrics, ${ }^{44,53,59,61,67,70,73-75,225-228}$ and superconductors. ${ }^{76,85}$ The general chemical formula of these materials is $\mathrm{ABO}_{2}$, where $\mathrm{A}$ is $\mathrm{a}+1$ metal cation like $\mathrm{Ag}, \mathrm{Cu}$ or $\mathrm{Pt}$ and $\mathrm{B}$ is a +3 metal cation like $\mathrm{Al}, \mathrm{Ga}$, In or $\mathrm{Co}$. In nature, these materials are found in two crystal forms, rhombohedral and hexagonal. Huda et al. showed that the symmetry of the ground state structure of these delafossites is related to the B-site element; ${ }^{69}$ delafossites with the B-site element in group IIIA of the periodic table tend to crystallize in rhombohedral symmetry $(\mathrm{R} \overline{3} \mathrm{~m})$ whereas the ones having B-site element belonging to group IIIB form structures with hexagonal symmetry $\left(\mathrm{P}_{3} / \mathrm{mmc}\right)$. In both of these polymorphs, B atoms are octahedrally coordinated with $\mathrm{O}$ atoms forming an infinite layer of $\mathrm{BO}_{6}$ units and $\mathrm{A}$ atoms are linearly coordinated with two $\mathrm{O}$ atoms. The existence of the two ploymorphs is 
a result of differences in the stacking of $\mathrm{BO}_{6}$ layers.

An interesting fact about group IIIA delafossites is that their optical gaps increase with the increasing atomic number. This is unexpected because in other compounds involving group IIIA elements, like $\mathrm{CuMS}_{2}$ and MAs $(\mathrm{M}=\mathrm{Al}, \mathrm{Ga}, \mathrm{In})$, the optically measured band gap decreases as the atomic number increases. Using DFT calculations Nie et al. showed that the fundamental direct band gaps in delafossites decreases with the increasing atomic number, however electron transitions across these gaps are forbidden due to HOMO and LUMO states having the same parity ${ }^{229}$. However, Huda found that using B-site doping, the inversion symmetry of these crystals can be broken, permitting photon absorption with the energy of the fundamental gap $^{95}$. After this discovery, several experiments suggested that this may also be true for Fe doped $\mathrm{CuGaO}_{2}{ }^{90,230}$.

According to Beznosikov and Aleksandrov, there are 9 A-site and 34 B-site candidates resulting in about 100 possible delafossite systems. ${ }^{231}$ As Huda and coworkers pointed out, B-site of delafossites can be doped with any other B-site element. ${ }^{95}$ This structural versatility gives rise to 13266 different delafossite structures. However, except for $\mathrm{Cu}$ family of delafossites, doping effects on the electronic structure of other delafossite systems have not been sufficiently explored. One such system is $\mathrm{Ag}$ delafossites which has been shown to possess opto-electronic properties suitable for photocatalytic applications. ${ }^{62,65,232,233}$ A possible reason for the lack of interest in calculations on doped delafossites could be the high computational costs involved with large supercells which are required to study the doped structures. In this work, 
we use FIREBALL to explore the structural and electronic properties of Fe-doped Ag delafossites. FIREBALL has been proven to have the capability to perform timeefficient calculations on supercells having number of atoms as large as $432 .{ }^{83,90,91,230}$

We start by analyzing the preferable Fe doping sites in $\mathrm{AgAl}_{1-x} \mathrm{Fe}_{x} \mathrm{O}_{2}$ and $\mathrm{AgGa}_{1-x} \mathrm{Fe}_{x} \mathrm{O}_{2}$ using two structural parameters. Next, we study the effects of doping on the valence and conduction band edge regions and how these effects are related to the structural changes. Finally, we show that Fe doping can result in electron transitions across the fundamental gap making Fe-doped Ag delafossites possible candidates for catalytic applications. To the best of our knowledge, this is the first work carried out on the B-site doping on silver family of delafossites.

\subsection{Computational Method}

All the calculations were performed with the FIREBALL ab-initio DFT package. ${ }^{104,106}$ In FIREBALL, kinetic and potential energy matrix elements are precomputed using a basis set derived from atomic orbitals. These orbitals have a definite cut-off radii (rc) values. For this study, we chose, $\mathrm{r}_{c}^{O}(\mathrm{~s})=3.40$ a.u. and $\mathrm{r}_{c}^{O}(\mathrm{p})=3.80$ a.u. for $\mathrm{O}$, $\mathrm{r}_{c}^{A l}(\mathrm{~s})=5.10$ a.u. and $\mathrm{r}_{c}^{A l}(\mathrm{p})=5.80$ a.u. for $\mathrm{Al}, \mathrm{r}_{c}^{G a}(\mathrm{~s})=4.80$ a.u. and $\mathrm{r}_{c}^{G a}(\mathrm{p})=5.70$ a.u. for Ga, $\mathrm{r}_{c}^{F e}(\mathrm{~s})=5.30$ a.u., $\mathrm{r}_{c}^{F e}(\mathrm{p})=5.80$ a.u. and $\mathrm{r}_{c}^{F e}(\mathrm{p})=4.70$ a.u. for Fe, $\mathrm{r}_{c}^{A g}(\mathrm{~s})$ $=5.30$ a.u., $\mathrm{r}_{c}^{A g}(\mathrm{p})=5.70$ a.u. and $\mathrm{r}_{c}^{A g}(\mathrm{~d})=4.60$ a.u. for Ag. We used local density approximation (LDA) exchange correlation functional, as previous studies carried out by our group and others have shown that structural and electronic property results obtained with LDA are in agreement with the experimental data. ${ }^{65,83,91,230}$ 
The crystal structures of both $\mathrm{AgAlO}_{2}$ and $\mathrm{AgGaO}_{2}$ were experimentally determined to exhibit rhombohedral symmetry $(\mathrm{R} \overline{3} \mathrm{~m})$. The rhombohedral until cell of the delafossites is shown in Figure 5.1 (solid lines), along with the equivalent hexagonal unit cell (drawn with dotted lines).

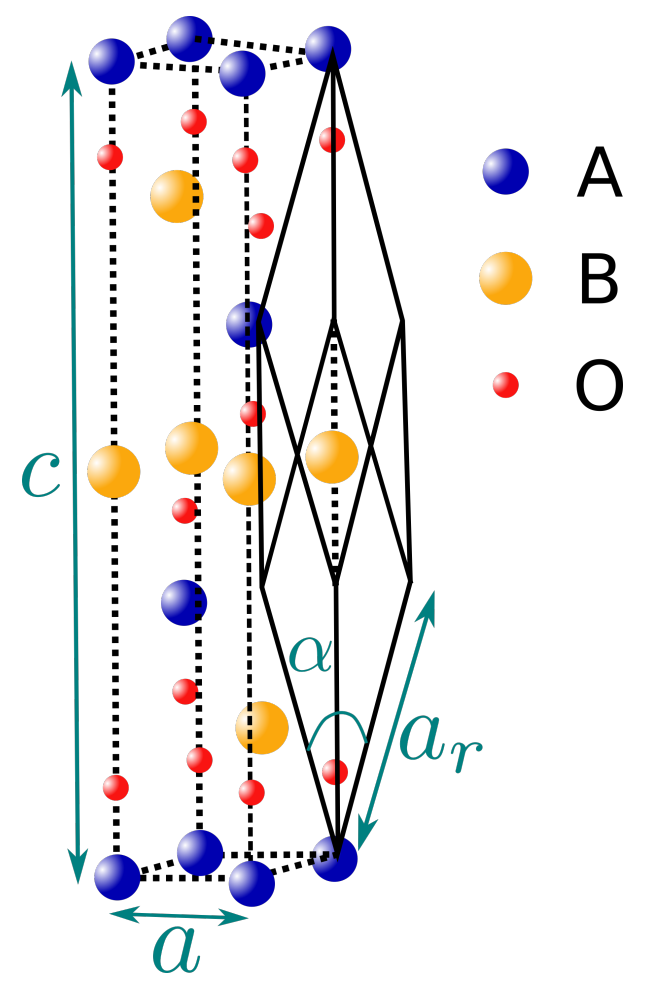

Figure 5.1: Equivalence between rhombohedral and hexagonal unit cells of delafossite systems. Rhombohedral unit cell is drawn with solid lines whereas the equivalent hexagonal representation is depicted using dotted lines.

The relationship between hexagonal and rhombohedral lattice constants can be given by, 


$$
\begin{gathered}
a_{r}=\frac{1}{3} \sqrt{c^{2}+3 a^{2}} \alpha \\
\alpha=\cos ^{-1}\left(\frac{2 c^{2}-3 a^{2}}{2 c^{2}+6 a^{2}}\right)
\end{gathered}
$$

where $a_{r}$ and $\alpha$ are rhombohedral lattice constants and $a$ and $c$ are hexagonal lattice constants.

Prior to performing calculations using precomputed matrix elements, it is necessary to confirm that they can reproduce results comparable to experimental quantities. We seek to achieve this by calculating the lattice constants of the two delafossite systems. The energy values corresponding to different a and c value pairs calculated by FIREBALL are given in Figure 5.2. For $\mathrm{AgAlO}_{2}$, a and c values of the lowest energy structure are, $2.9874 \AA$ and $19.253 \AA$ and the corresponding experimental values are $2.8729 \AA$ are $18.336 \AA .{ }^{45}$ FIREBALL-obtained a and c values for $\mathrm{AgGaO}_{2}$ are $2.9889 \AA$ and $18.905 \AA$, whereas the corresponding experimental values are $2.9889 \AA$ and $18.534 \AA{ }^{45}$ These results validate our calculated matrix elements as the differences between calculated and experimental lattice constants are between $1-5 \%$ of the experimental values. 


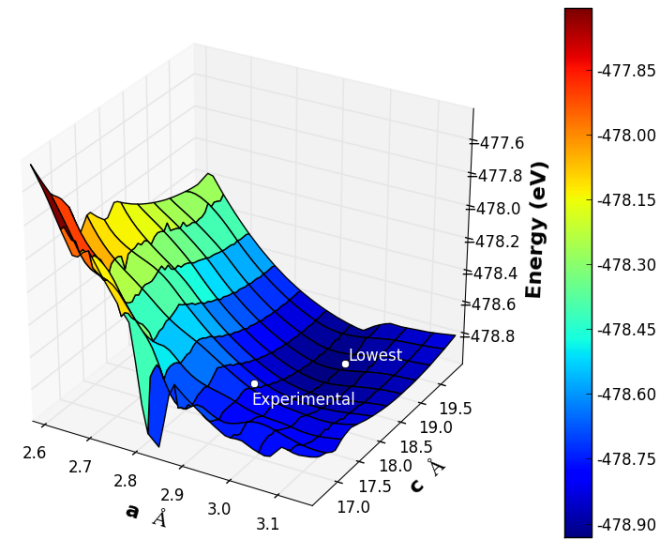

(a)

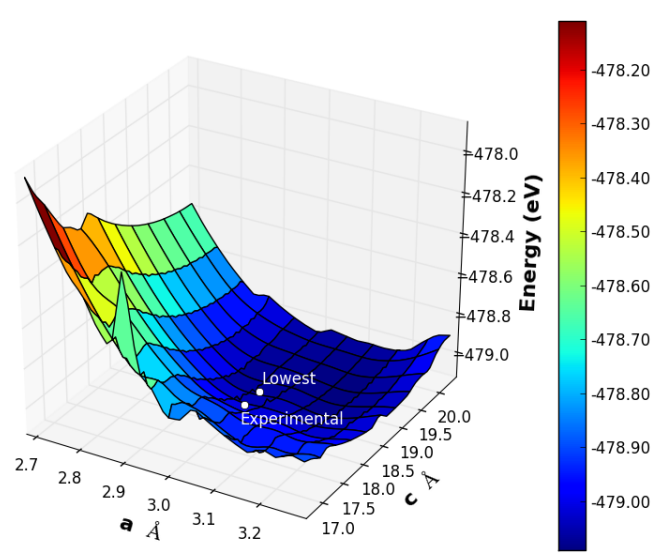

(b)

Figure 5.2: Total energy surfaces of (a) $\mathrm{AgAlO}_{2}$ and (b) $\mathrm{AgGaO}_{2}$ corresponding to different $a$ and $c$ lattice constants.

In order to get a realistic picture on preferable dopant locations, it is necessary to consider a sufficiently large supercell. Smaller supercells force dopants to distribute in different B-site layers when the number of dopants is large. Using the four-atom unit cell having the experimental lattice constants, ${ }^{44}$ we created an equivalent 12 atom unit cell in the equivalent and convenient hexagonal representation. This unit cell is then repeated in the $\mathrm{x}, \mathrm{y}$ and $\mathrm{z}$ directions to obtain a 432 atom supercell, as shown in Figure 5.3. 


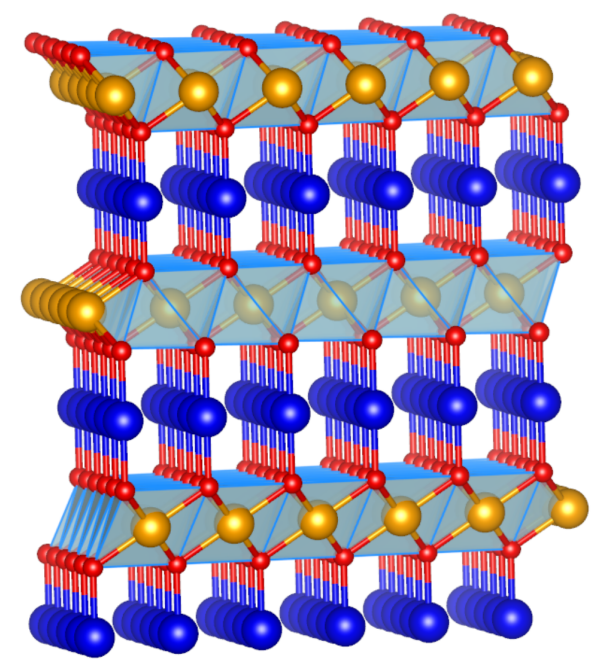

Figure 5.3: Supercell created by repeating the 12-atom hexagonal unit cell.

For $2 \%$ Fe doping we considered all the 107 possible doped structures. For $3 \%$ - 5\% Fe doping, 500 supercells with random Fe doping sites were created. Vegards' law (Equation 5.1) was imposed to take the scaling of the lattice constants into account. ${ }^{234}$ In Equation 5.1, $a$ is the modified lattice constant of $\mathrm{AgBO}_{2}(\mathrm{~B}=\mathrm{Al} / \mathrm{Ga})$ when it is doped with $x$ percent of Fe.

$$
a=(1-x) \times a_{\mathrm{AgBO}_{2}}+x \times a_{\mathrm{AgFeO}_{2}}
$$

As the size of the supercell is large, all the structures were relaxed at the $\Gamma$ point until the root-mean-square force on atoms is less than $0.05 \mathrm{eV} / \AA$. In the calculation of electronic density of states, selected lowest energy structures after relaxation were subjected to one self-consistent-field (SCF) calculation using a $2 \times 2 \times 2$ Monkhorst-Pack k-point grid. 
We used two parameters to analyze the positioning of the dopants in the supercell: clustering and layering factors. Clustering factor measures how closely the dopant atoms are positioned with respect to each other. Clustering factor for a particular dopant $\left(\mathrm{CF}_{i}\right)$ is defined as, ${ }^{83}$

$$
C F_{i}=\frac{1}{n-1} \sum_{j \neq i}^{n-1}\left(d_{i, j}-d_{0}\right)^{2}
$$

Here, $\mathrm{d}_{i, j}$ is the distance between dopant atom $i$ and its neighboring dopant $j$ and $n$ is the total number of dopants. The distance between two nearest neighbor Bsite atoms in the undoped structure is given by $\mathrm{d}_{0}$. The difference $\mathrm{d}_{i, j}-\mathrm{d}_{0}$ compares the distance between two dopants with the nearest neighbor B-site distance $d_{0}$ in the undoped structure. Therefore, $\mathrm{CF}_{i}$ values closer to zero indicate that the dopant atoms are positioned as nearest neighbors. The clustering factor of the supercell $(\mathrm{CF})$ is defined as the minimum of $\mathrm{CF}_{i}$ values calculated for all the $\mathrm{n}$ number of dopants atoms.

$$
C F=\min \left\{C F_{1}, C F_{2}, \ldots . C F_{n}\right\}
$$

We defined the layering factor of the supercell as the maximum of the dopant percentage in any of the $(0,0,0,1)$ B-site planes as a percentage of $n$,

$$
L F=\max \left\{L F_{1}, L F_{2}, L F_{3}\right\} \times 100 / n
$$

Here, $\mathrm{LF}_{i}$ is the the number of dopant atoms in the $\mathrm{i}^{\text {th }} \mathrm{B}$-site plane of the supercell. As shown in Figure 5.3 there are only three B-site planes in our supercell. 


\subsection{Results and discussion}

In the following discussions on the structural and electronic properties, we only considered the ten lowest energy structures.

\subsubsection{Structural properties of $\mathrm{AgAl}_{1-x} \mathrm{Fe}_{x} \mathrm{O}_{2}(\mathrm{x}=2 \%-5 \%)$}

Interpreting the clustering and layering factors together helps us to get a comprehensive picture on the arrangement of the Fe atoms in the supercell. Clustering factor results for $\mathrm{x}=2 \%$ and $\mathrm{x}=3 \%$ shows that lowest energy structures have the lowest clustering factors observed for those doping percentages. In fact, the clustering factors of most of the $2 \%$ doped structures are close to zero which indicates that Fe substitutions occur at nearest neighbor Al sites. We also see from Figure 5.4 (a) that for $2 \%$ and $3 \%$ of Fe doping, large clustering factors or large Fe-Fe distances are associated with high structural energies. For example, in the lowest energy structure for $\mathrm{x}=3 \%$, there are two pairs of nearest-neighbor Fe atoms and one pair of Fe atoms of which the Fe-Fe distance is equal to the $2^{\text {nd }}$ nearest neighbor distance (Figure C.4, Appendix C). However, as the structural energy increases, only one pair of nearest neighbor Fe atoms is present in the supercell and the majority of the Fe-Fe bond lengths are greater than the $2^{\text {nd }}$ nearest neighbor B-site distance. Another interesting observation in $2 \%$ and $3 \%$ Fe-doped structures is that the preferable dopant sites lie on the same $(0,0,0,1)$ plane as indicted by layering factors of $100 \%$ obtained for most of the low energy structures. 
Similar to $\mathrm{x}=2 \%$ and $\mathrm{x}=3 \%$ results, lowest energy $4 \%$ Fe-doped structure is also associated with the lowest clustering factor. All the Fe atoms in this structure occupy the same plane as two pairs of nearest neighbors (see Figure C.3 in APPENDIX C). These two pairs are also located at a close proximity to each other, giving rise to the low clustering factor. However, in the next three lowest energy structures for $\mathrm{x}=4 \%$, Fe atoms are arranged in two-in-one-and-two-in-another-plane configuration resulting in layering factors smaller than $100 \%$. We should also note the existence of four other structures with all four Fe atoms in the same plane. The energy differences between these structures and the lowest energy structure for $\mathrm{x}=$ $4 \%$ are only around 0.4-0.6 eV. This shows that all the dopants occupying the same plane is preferable for the case $\mathrm{x}=4 \%$.

Most of the 5\% Fe doped structures are associated with high clustering factors indicating Fe substitutions occurring at non-nearest neighbor $\mathrm{Al}$ sites. According to the layering factors, in the two lowest energy structures, four Fe atoms are on the same plane and one in a different plane (see Figure C.4 in APPENDIX C). However, the energy of the structure with all the five Fe atoms in the same plane is only 0.05 $\mathrm{eV}$ greater than that of the $5 \%$ doped lowest energy structure. This indicates that the all the dopants in the same plane configuration is also favorable.

In summary, structural trends for $\mathrm{AgAl}_{1-x} \mathrm{Fe}_{x} \mathrm{O}_{2}$ shows that in particular for $\mathrm{x}<4 \%$, Fe dopants prefer to get substituted for $\mathrm{Al}$ sites on the same plane, which are at close proximity to each other. 


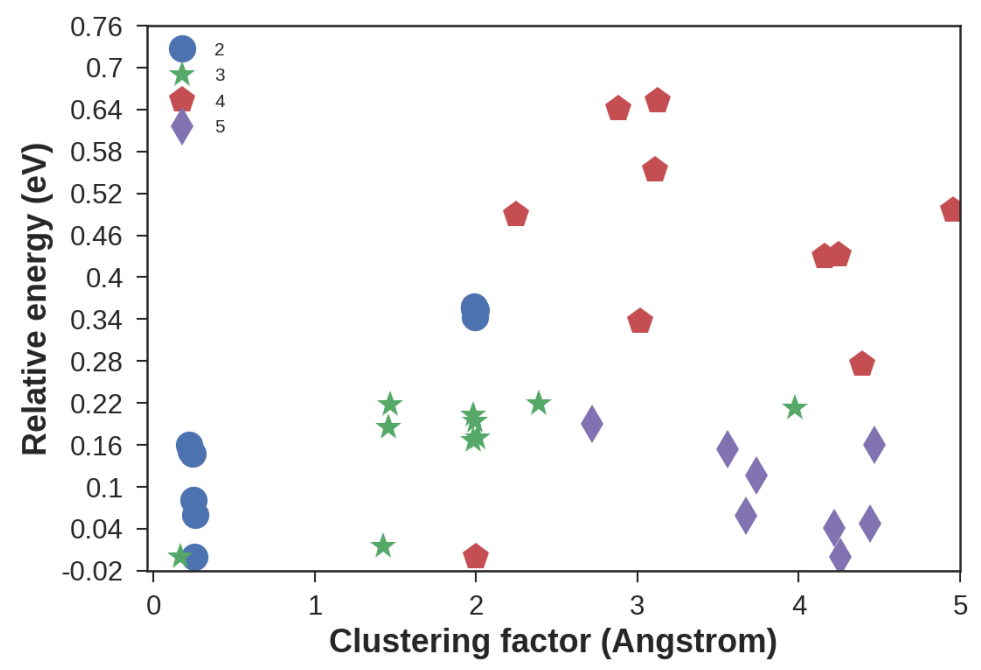

(a)

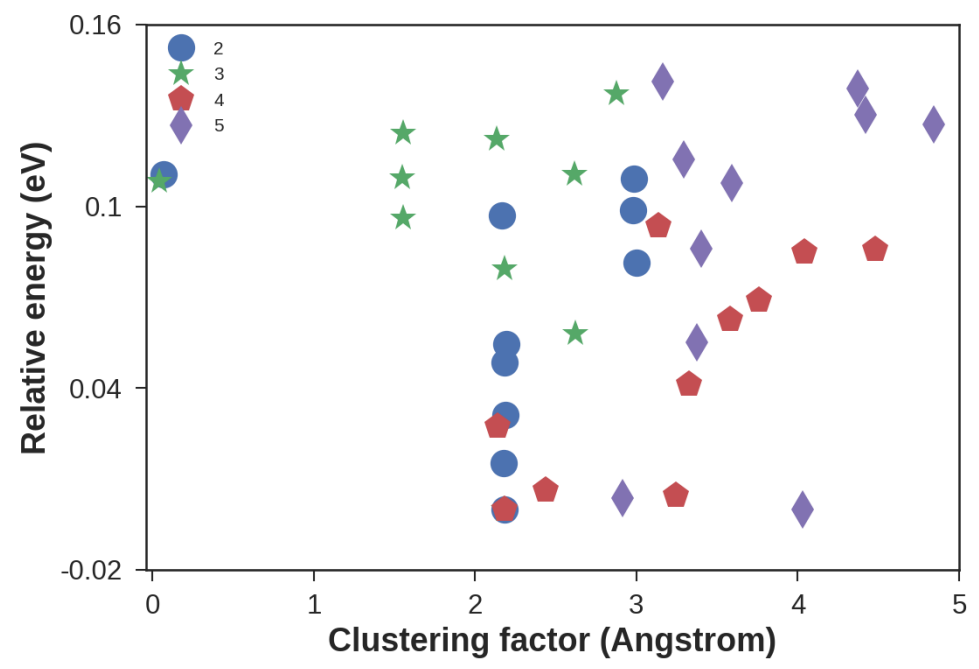

(b)

Figure 5.4: Clustering factor results of (a) $\mathrm{AgAl}_{1-x} \mathrm{Fe}_{x} \mathrm{O}_{2}$ and (b) $\mathrm{AgGa}_{1-x} \mathrm{Fe}_{x} \mathrm{O}_{2}$ for $x=2 \%-5 \%$. Relative energy is defined as the difference between energy of a particular structure and the energy of the lowest energy structure for a given doping percentage (E$\left.\mathrm{E}_{\min }\right)$. 


\subsubsection{Structural properties of $\mathrm{AgGa}_{1-x} \mathrm{Fe}_{x} \mathrm{O}_{2}(x=2 \%-5 \%)$}

Unlike in $\mathrm{AgAl}_{1-x} \mathrm{Fe}_{x} \mathrm{O}_{2}$, clustering factors of the lowest energy $2 \%$ and $3 \% \mathrm{Fe}$ doped $\mathrm{AgGa}_{1-x} \mathrm{Fe}_{x} \mathrm{O}_{2}$ are not close to zero. This result shows that for $\mathrm{x} \leq 3 \%$ Fe-Fe distances in energetically favorable structures are greater than the nearest neighbor B-site distance. Nearest neighbor Fe dopants are observed in the ninth and third lowest energy structures for $\mathrm{x}=2 \%$ and $\mathrm{x}=3 \%$ calculations respectively. The fact that their relative energies are only about $0.01 \mathrm{eV}$ indicates that nearest neighbor Fe substitution is also not unstable. However, as the clustering factors of majority of the $2 \%$ and $3 \%$ doped structures are greater than 3 , we can conclude that for low amounts of Fe doping, clustering of Fe atoms within a close proximity to each other is not energetically favorable in $\mathrm{AgGa}_{1-x} \mathrm{Fe}_{x} \mathrm{O}_{2}$. A similar result has also been obtained by Haycock et al., for $\mathrm{CuGa}_{1-x} \mathrm{Fe}_{x} \mathrm{O}_{2} \cdot{ }^{83}$ We suspect the lower clustering factor in the $\mathrm{AgAl}_{1-x} \mathrm{Fe}_{x} \mathrm{O}_{2}$ supercells to be due to a combination of two properties of $\mathrm{Al}$, which are $\mathrm{Al}$ is somewhat metallic in nature, and that the atomic size of $\mathrm{Al}$ is much smaller than that of $\mathrm{Fe}$.

The general trend in the clustering factors for $\mathrm{x}=4 \%$ and $\mathrm{x}=5 \%$ is that, the clustering factor increases with the increasing relative energy. This behavior of the clustering factors can be linked with the corresponding layering factor values. According to the layering factors of $\mathrm{AgGa}_{1-x} \mathrm{Fe}_{x} \mathrm{O}_{2}$, dopant atoms tend to occupy different planes as the doping percentage increases beyond 3. Fe dopants in different planes causes Fe-Fe distances to increase, resulting in large clustering factors. It is also notable that, given by the layering factors greater than $70 \%$ in most of the 
low energy structures for $\mathrm{x}=4 \%$ and $\mathrm{x}=5 \%$, all the dopants occupying the same plane can be considered favorable. Positions of Fe atoms in selected $\mathrm{AgGa}_{1-x} \mathrm{Fe}_{x} \mathrm{O}_{2}$ structures are shown in Figures C.7 to C.10 in Appendix C. 

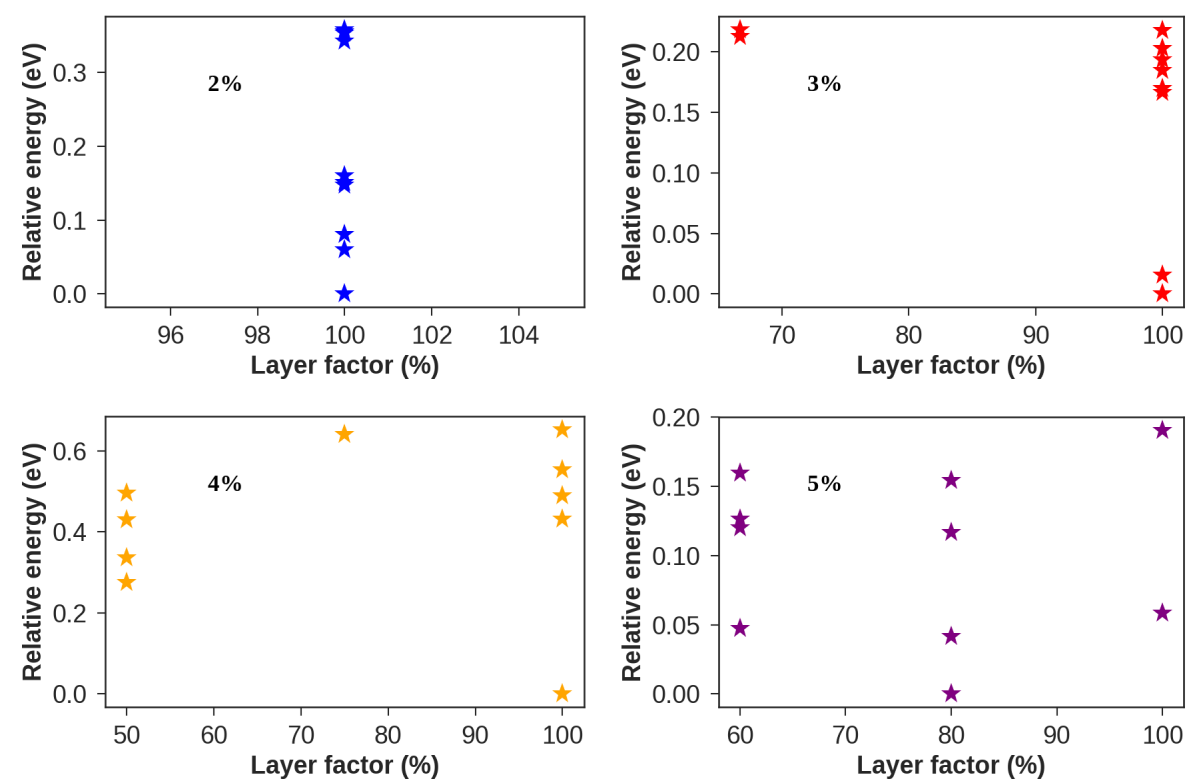

(a)
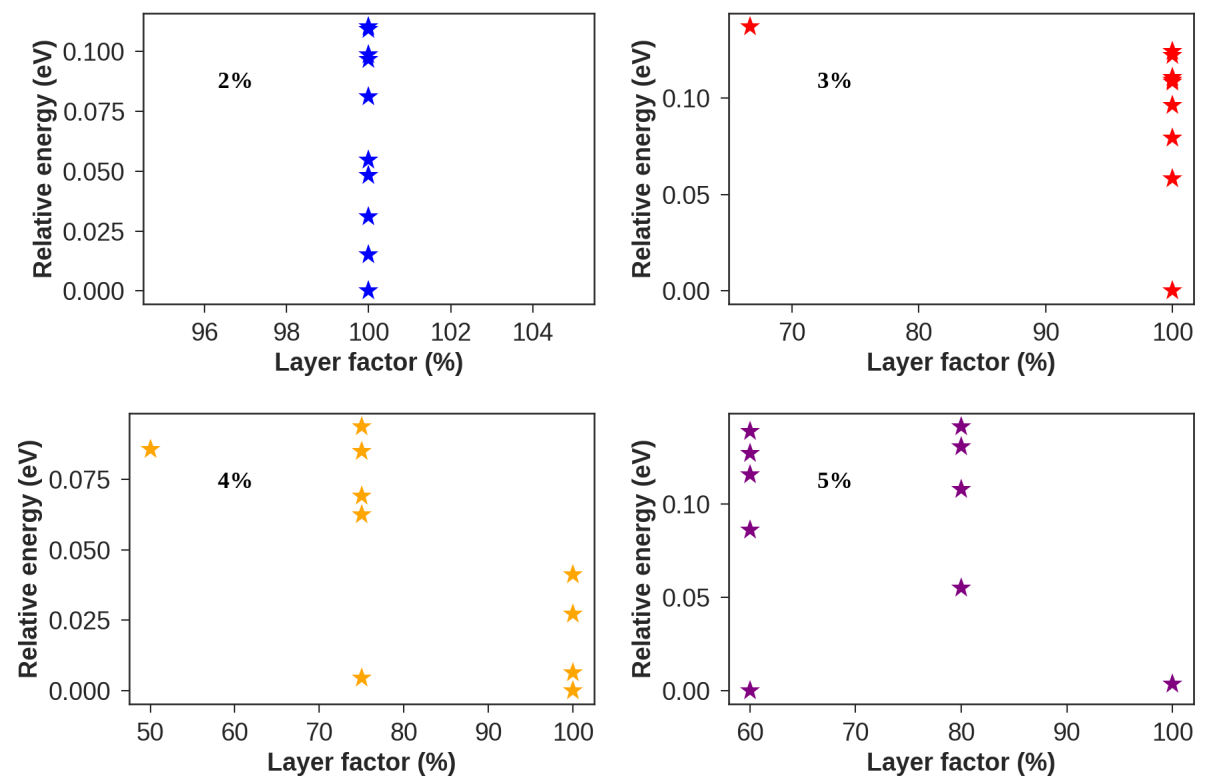

(b)

Figure 5.5: Layering factor results of (a) $\mathrm{AgAl}_{1-x} \mathrm{Fe}_{x} \mathrm{O}_{2}$ and (b) $\mathrm{AgGa}_{1-x} \mathrm{Fe}_{x} \mathrm{O}_{2}$ 


\subsubsection{Electronic properties}

The electronic band structures of $\mathrm{AgAlO}_{2}$ and $\mathrm{AgGaO}_{2}$ are shown in Figure 5.6. Possible electronic transitions at $\Gamma$ and $\mathrm{M}$ points are listed in Table 5.1 together with the experimentally obtained optical gaps.
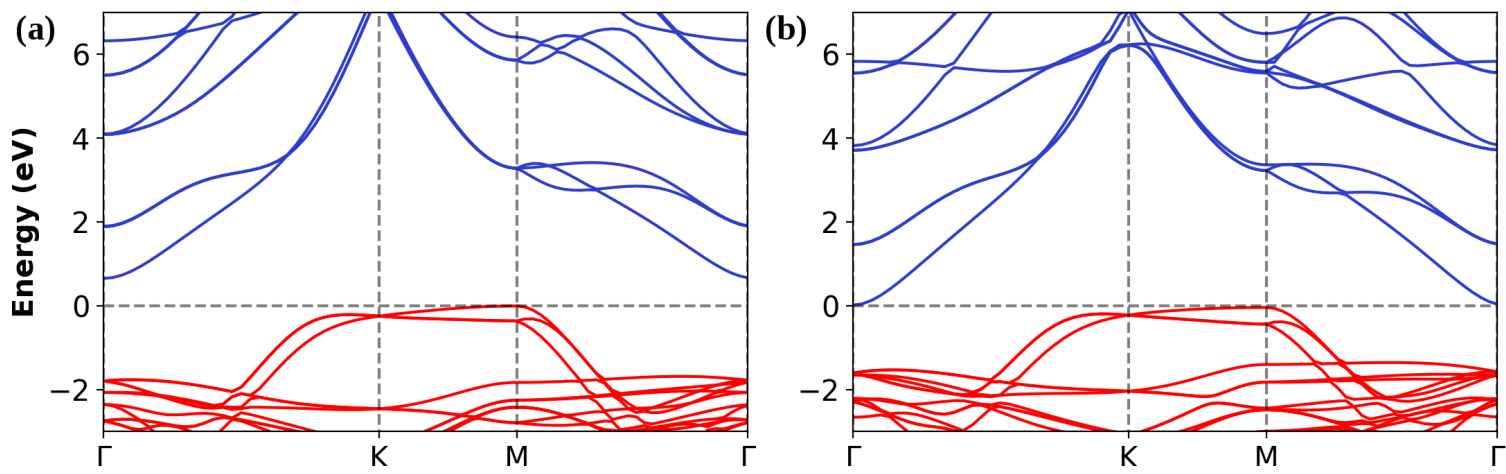

Figure 5.6: Electronic band structures of (a) $\mathrm{AgAlO}_{2}$ and (b) $\mathrm{AgGaO}_{2}$. Occupied and unoccupied bands are colored in red and blue respectively. Valence band maximum is set to $0 \mathrm{eV}$.

\begin{tabular}{|c|c|c|c|}
\hline & $\Gamma^{\text {direct }}(\mathrm{eV})$ & $M^{\text {direct }}(\mathrm{eV})$ & Optical \\
\hline $\mathrm{AgAlO}_{2}$ & 2.4 & 3.26 & 3.6 \\
\hline $\mathrm{AgGaO}_{2}$ & 1.6 & 3.29 & 4.1 \\
\hline
\end{tabular}

Table 5.1: Calculated band gaps in comparison with the optical gaps

For both $\mathrm{AgAlO}_{2}$ and $\mathrm{AgGaO}_{2}$, the valence band maximum is at the $\mathrm{M}$ point and the conduction band minimum is at the $\Gamma$ point. The computed direct band gaps 
at the $\Gamma$ point for $\mathrm{AgAlO}_{2}$ and $\mathrm{AgGaO}_{2}$ decreases from $2.4 \mathrm{eV}$ to $1.6 \mathrm{eV}$. This reduction in the gap when the $\mathrm{B}$ site changes from $\mathrm{Al}$ to Ga agrees with the trends in other group 13 containing compounds ${ }^{235}$. The computed direct gap at the M point for $\mathrm{AgAlO}_{2}$ is close to the optical gap obtained by Sheets et al ${ }^{65}$. Even though Sheets et al., synthesized $\mathrm{AgGaO}_{2}$, they did not report its optical gap due to difficulties caused by additional light absorption by $\mathrm{AgGaO}_{2}$. The other reported optical gap of 4.1 eV was by Vanaja et al. ${ }^{62}$. However, $\mathrm{AgGaO}_{2}$ that they have prepared belongs to a phase that is different from that of $\mathrm{AgAlO}_{2}$ synthesized by Sheets et al. Therefore, it is difficult to make a reasonable comparison between experimental and calculated optical gaps for $\mathrm{AgGaO}_{2}$.

We now discuss the changes in the electronic properties of these delafossites due to Fe doping using electronic density of states (DOS) averaged over 10 lowest energy structures (when $\mathrm{x} \geq 2$ ). The main change due the Fe doping in both $\mathrm{AgAl}_{1-x} \mathrm{Fe}_{x} \mathrm{O}_{2}$ and $\mathrm{AgGa}_{1-x} \mathrm{Fe}_{x} \mathrm{O}_{2}$ is the shift of the valence band edge toward higher energies (Figure 5.7). This effect is also found in Fe doped cuprite delafossites ${ }^{95}$. 

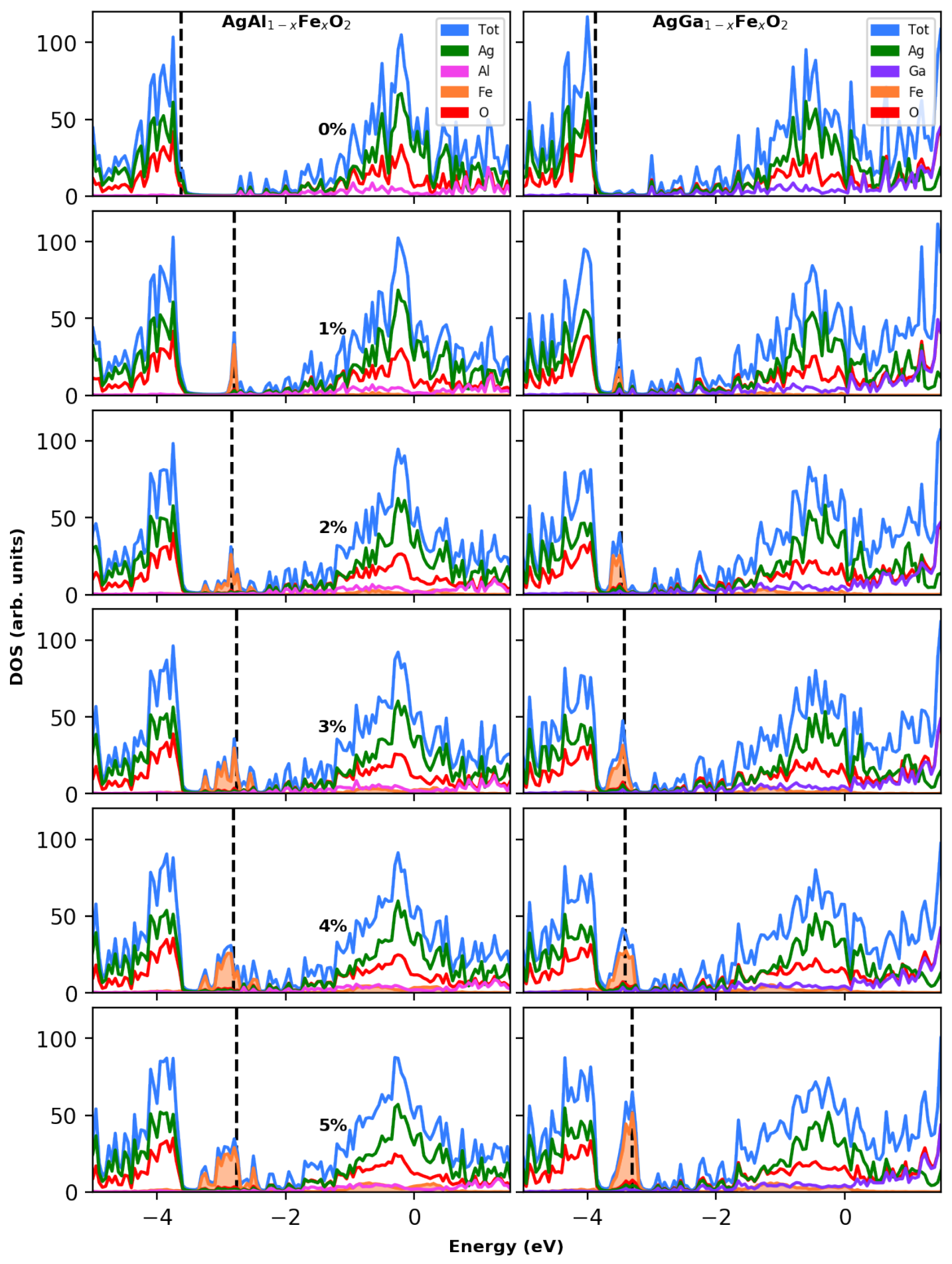

Figure 5.7: Average PDOS of $\mathrm{AgAl}_{1-x} \mathrm{Fe}_{x} \mathrm{O}_{2}$ (left panel) and $\mathrm{AgGa}_{1-x} \mathrm{Fe}_{x} \mathrm{O}_{2}$ (right panel) 
As shown in Figures 5.7, 5.8 (a) and 5.9 (a), the valence band edge of both $\mathrm{AgAlO}_{2}$ and $\mathrm{AgGaO}_{2}$ is mainly composed of $\mathrm{O}-\mathrm{p}$ and $\mathrm{Ag}-\mathrm{d}$ states. Introduction of one $\mathrm{Fe}$ atom creates $\mathrm{Fe}-\mathrm{d}$ states around $-2.8 \mathrm{eV}$ in $\mathrm{AgAl}_{0.99} \mathrm{Fe}_{0.01} \mathrm{O}_{2}$, forming the new valence band edge. Increasing the Fe percentage beyond 2 creates new $\mathrm{Fe}$ states delocalized around $-2.8 \mathrm{eV}$. Valence band edges of both $\mathrm{AgAl}_{0.99} \mathrm{Fe}_{0.01} \mathrm{O}_{2}$ and $\mathrm{AgGa}_{0.99} \mathrm{Fe}_{0.01} \mathrm{O}_{2}$ are predominantly composed of Fe states. Some hybridization of Fe-d states with O-p and Ag-d states is also visible in both cases (Figures 5.8 (b) and $5.9(\mathrm{~b}))$. However, when the Fe percentage is at 2 and above, the valence band edge is solely dominated by Fe states and the Fe hybridization with $\mathrm{Ag}$ and $\mathrm{O}$ states is negligible. Unlike in $\mathrm{AgAl}_{1-x} \mathrm{Fe}_{x} \mathrm{O}_{2}$, Fe states in $\mathrm{AgGa}_{1-x} \mathrm{Fe}_{x} \mathrm{O}_{2}$ remain localized around $-3.7 \mathrm{eV}$ as the Fe percentage increases. For both delafossites, the largest shift in the valence band edge occurs when Fe percentage changes from 0 to 1: $0.8 \mathrm{eV}$ in $\mathrm{AgAl}_{1-x} \mathrm{Fe}_{x} \mathrm{O}_{2}$ and $0.2 \mathrm{eV}$ in $\mathrm{AgGa}_{1-x} \mathrm{Fe}_{x} \mathrm{O}_{2}$. Subsequent additions of $\mathrm{Fe}$ atoms have little effect on the energy corresponding to the valence band edge. At $5 \%$ of $\mathrm{Fe}$ doping, the shifts in the valence band edge compared to the undoped case are 0.86 $\mathrm{eV}$ and $0.4 \mathrm{eV}$ for $\mathrm{AgAl}_{1-x} \mathrm{Fe}_{x} \mathrm{O}_{2}$ and $\mathrm{AgGa}_{1-x} \mathrm{Fe}_{x} \mathrm{O}_{2}$ respectively. 


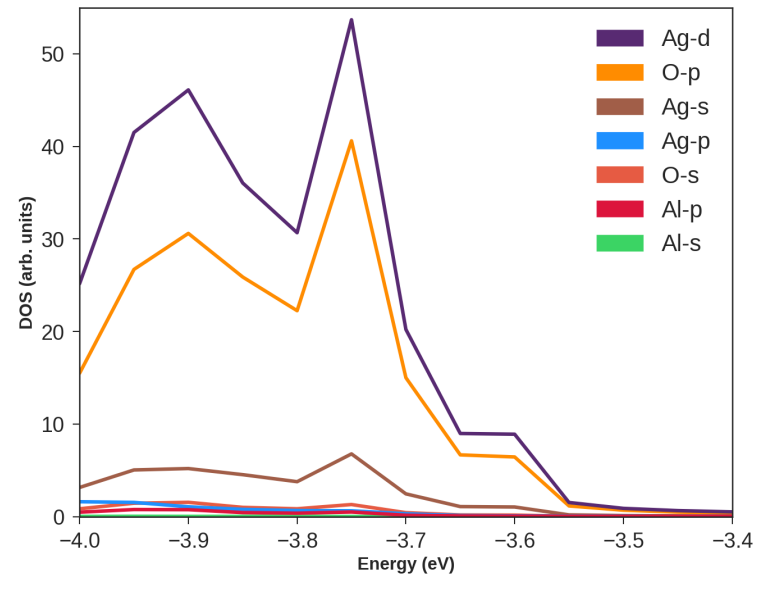

(a)

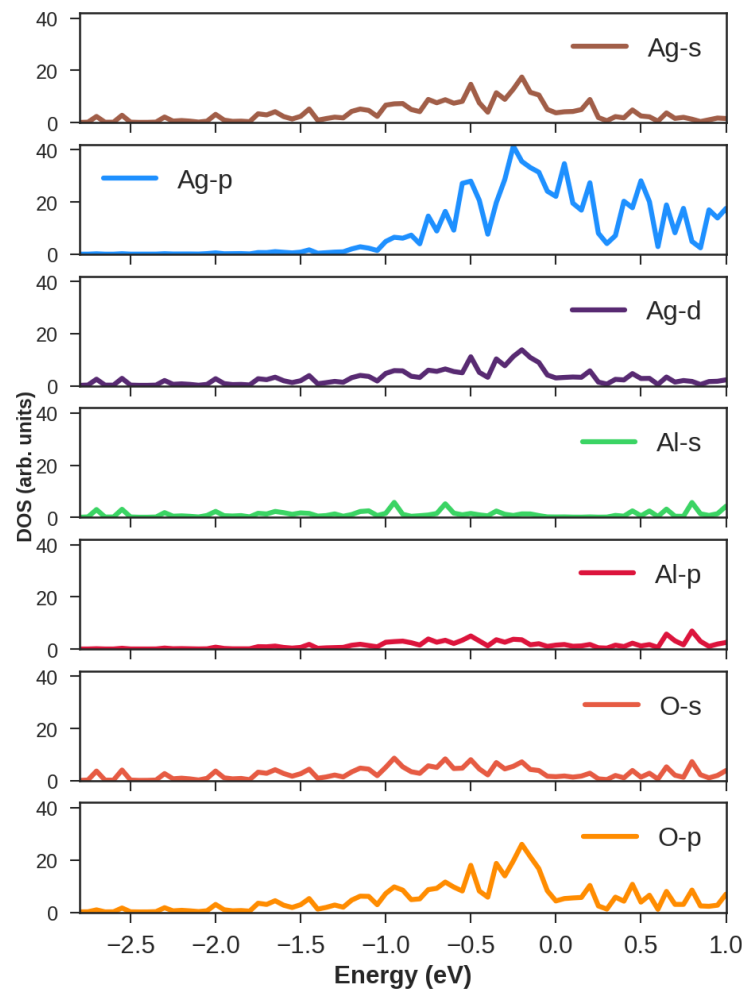

(c)

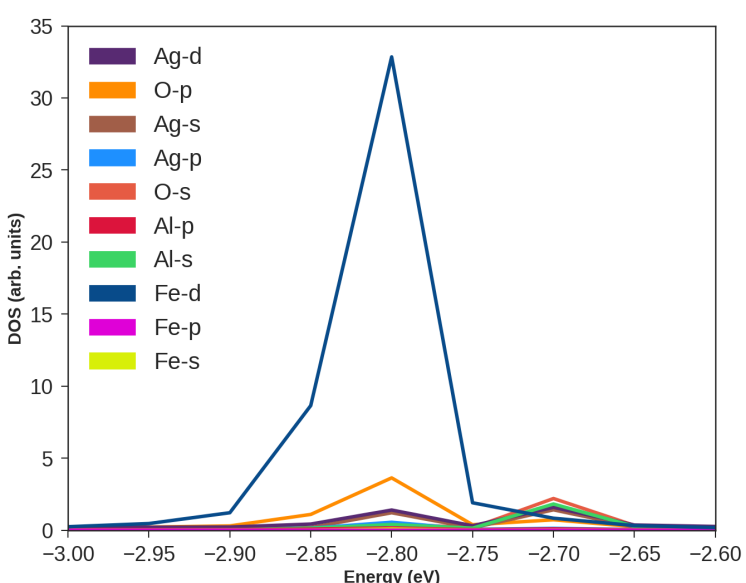

(b)

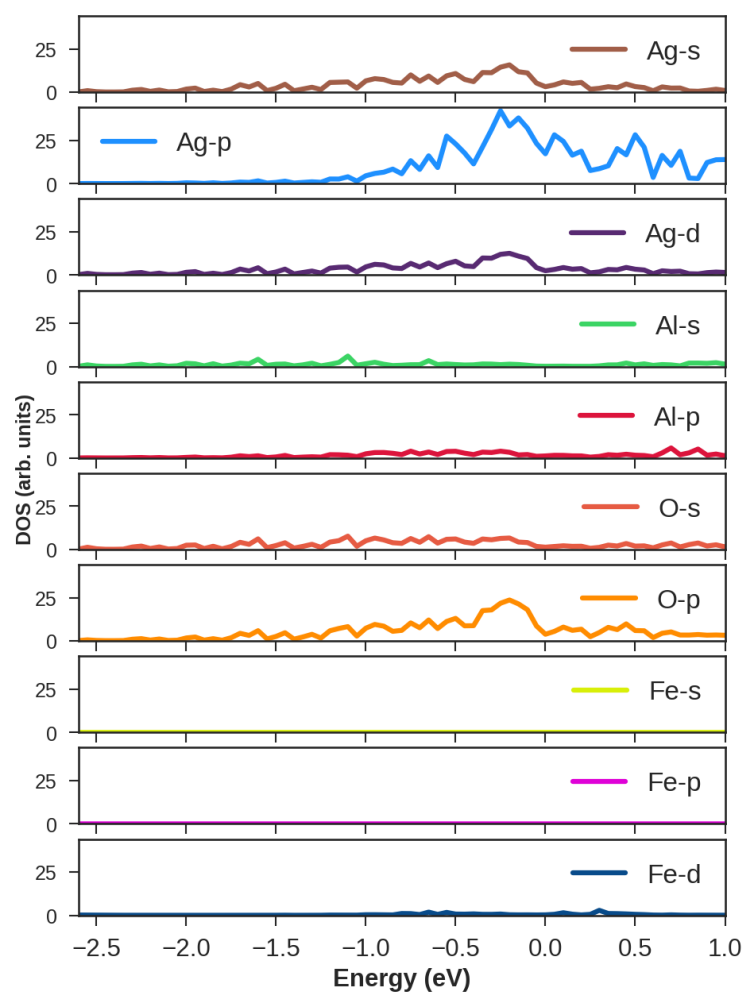

(d)

Figure 5.8: Orbital DOS at the valence band edge of (a) $\mathrm{AgAlO}_{2}$ and (b) $\mathrm{AgAl}_{0.99} \mathrm{Fe}_{0.01} \mathrm{O}_{2}$ and at the conduction band region of (c) $\mathrm{AgAlO}_{2}$ and (d) $\mathrm{AgAl}_{0.99} \mathrm{Fe}_{0.01} \mathrm{O}_{2}$ 


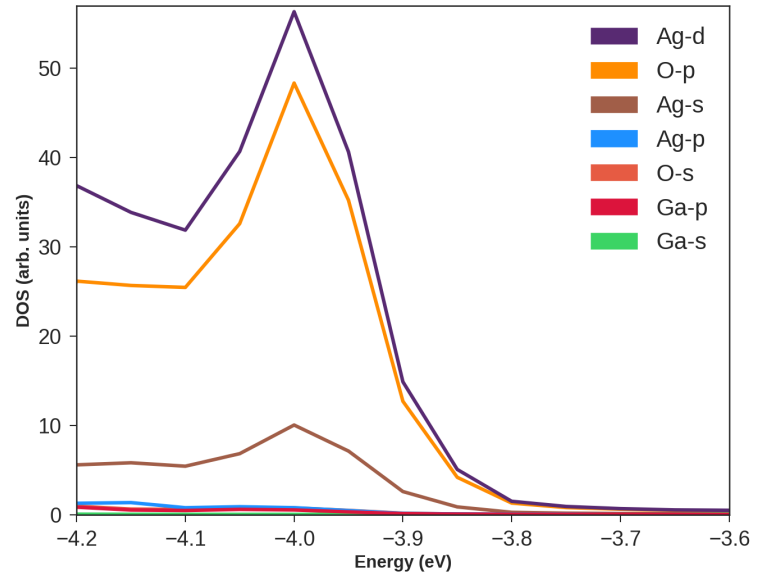

(a)

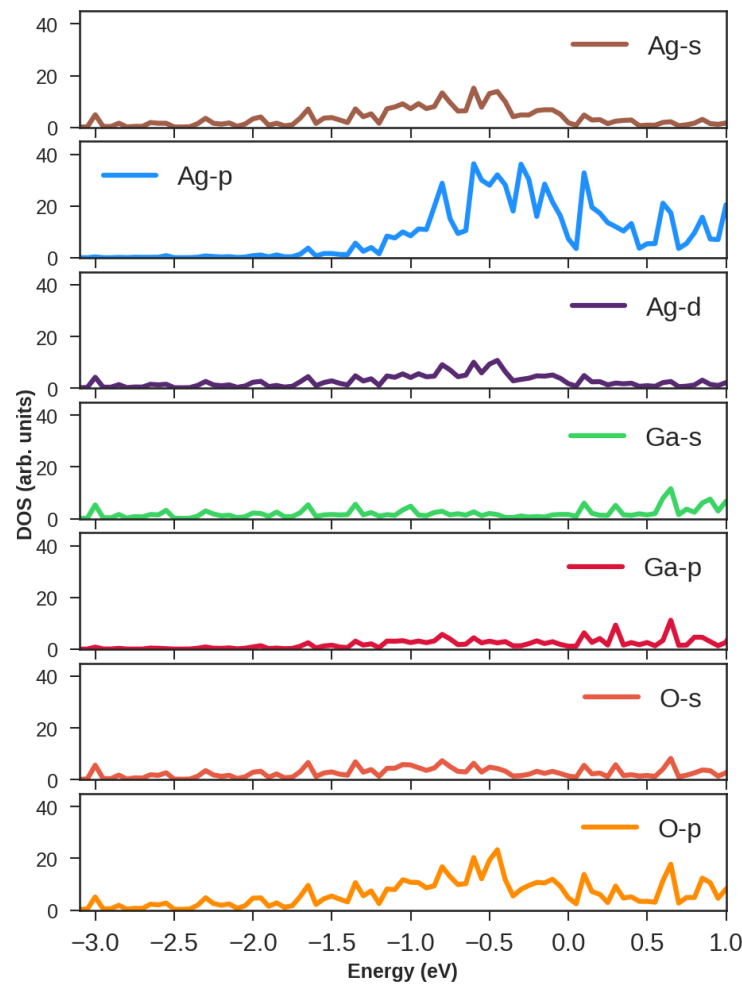

(c)

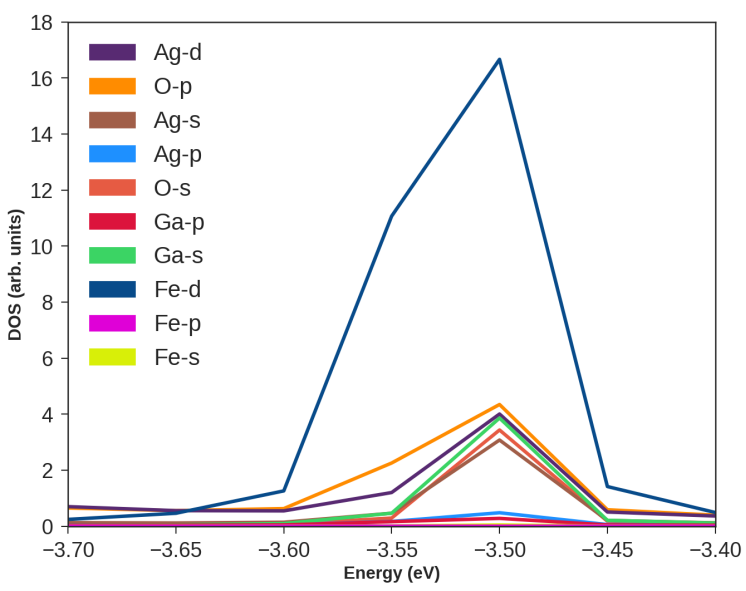

(b)

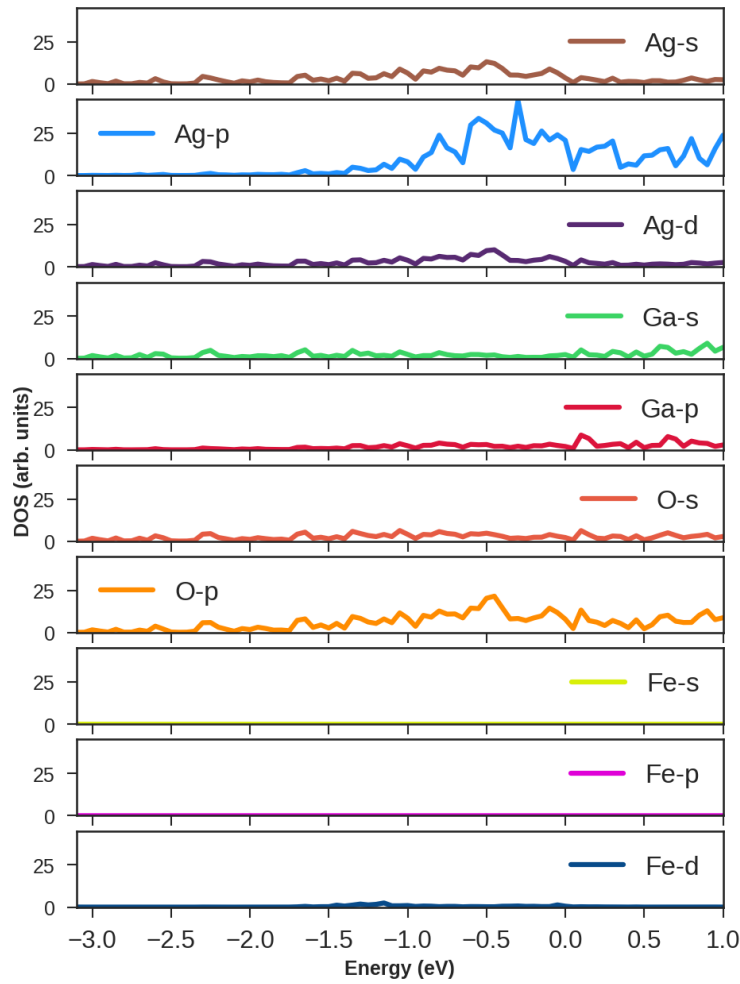

(d)

Figure 5.9: Orbital DOS at the valence band edge of (a) $\mathrm{AgGaO}_{2}$ and (b) $\mathrm{AgGa}_{0.99} \mathrm{Fe}_{0.01} \mathrm{O}_{2}$ and at the conduction band region of (c) $\mathrm{AgGaO}_{2}$ and (d) $\mathrm{AgGa}_{0.99} \mathrm{Fe}_{0.01} \mathrm{O}_{2}$ 
Inspection of partial density of states (PDOS) of $\mathrm{AgAl}_{0.98} \mathrm{Fe}_{0.02} \mathrm{O}_{2}$ revealed that the Fe states in the valence band region form three main peaks (centered at -2.5 $\mathrm{eV},-3.0 \mathrm{eV}$ and $-3.25 \mathrm{eV}$ ) when Fe atoms are at nearest neighbors, and only one main peak (around $-2.8 \mathrm{eV}$ ) when the Fe atoms are separated by a distance of two nearest neighbors (see Figure 5.10). PDOS corresponding to these two cases are given in Figure 5.10. This relationship between the Fe peaks and the Fe-Fe distance allows us to correlate the broadening of the Fe states with the clustering factors as depicted in Figure 5.10. The presence of broadened Fe states is associated with small clustering factors. Thus for $2 \% \mathrm{AgAl}_{0.98} \mathrm{Fe}_{0.02} \mathrm{O}_{2}$, clustering factor is inversely proportional to the broadening observed in the valence band region. However, this phenomenon was not observed in $\mathrm{AgGa}_{0.98} \mathrm{Fe}_{0.02} \mathrm{O}_{2}$. 

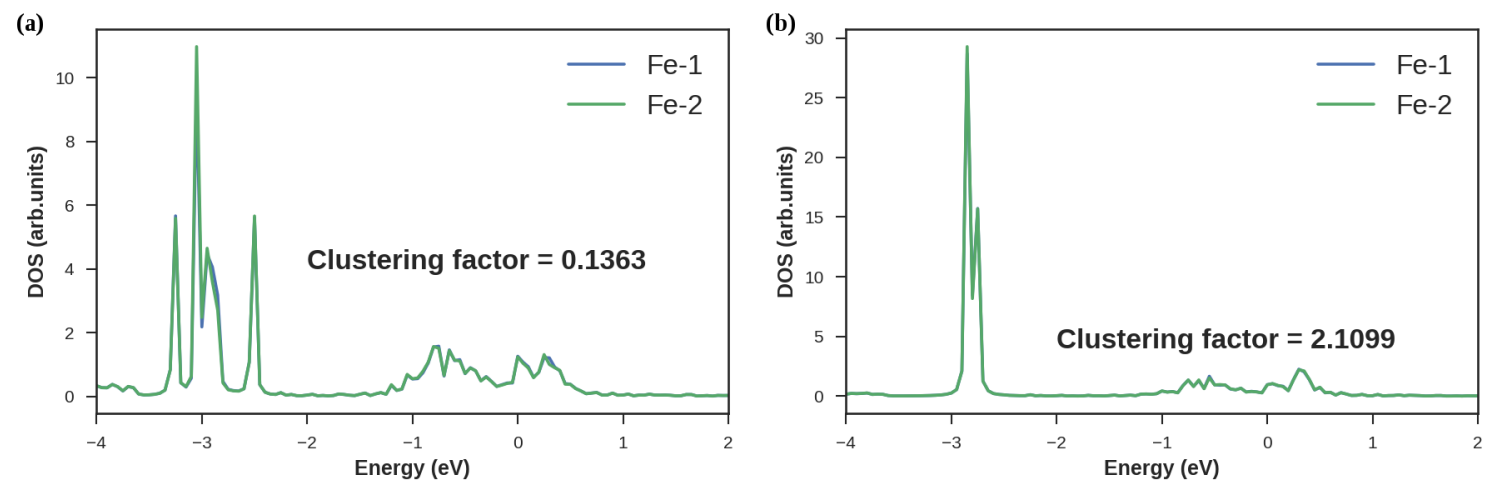

Figure 5.10: PDOS in (a) correspond to a $\mathrm{AgAl}_{0.98} \mathrm{Fe}_{0.02} \mathrm{O}_{2}$ supercell where the two $\mathrm{Fe}$ atoms are separated by a distance of two nearest neighbors. The valence band edge consists of only one peak around $-3 \mathrm{eV}$ as seen in (a). However, when the Fe atoms are nearest neighbors, valence band edge is split into two main peaks about $-3 \mathrm{eV}$

Another interesting trend in the density of Fe states is observed in both these delafossites, when there are 3 or more Fe atoms. When a Fe atom is located relatively far away from the rest, most of the Fe density is concentrated on this isolated Fe atom. Thus, for $\mathrm{x} \geq 3 \%$, when there is an isolated Fe atom, the valence band edge is mainly composed of states contributed by that Fe atom. Examples of this effect are given in the Figures 5.11 and 5.12. 


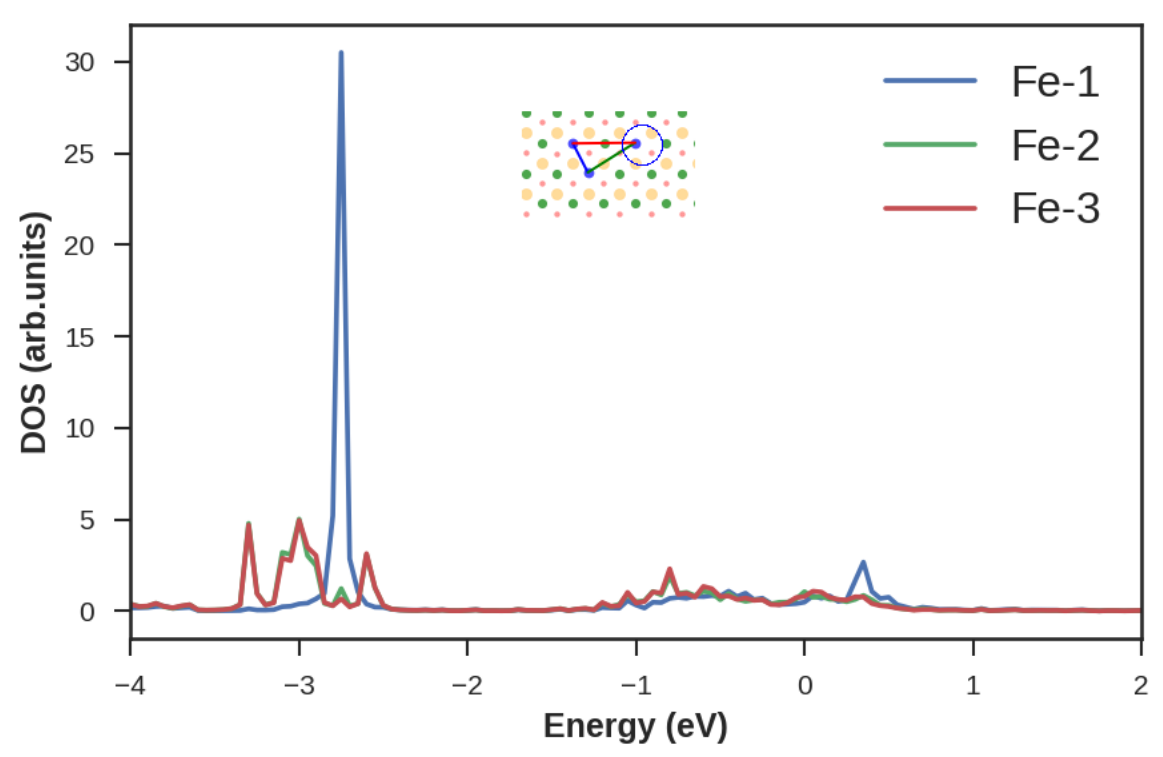

(a)

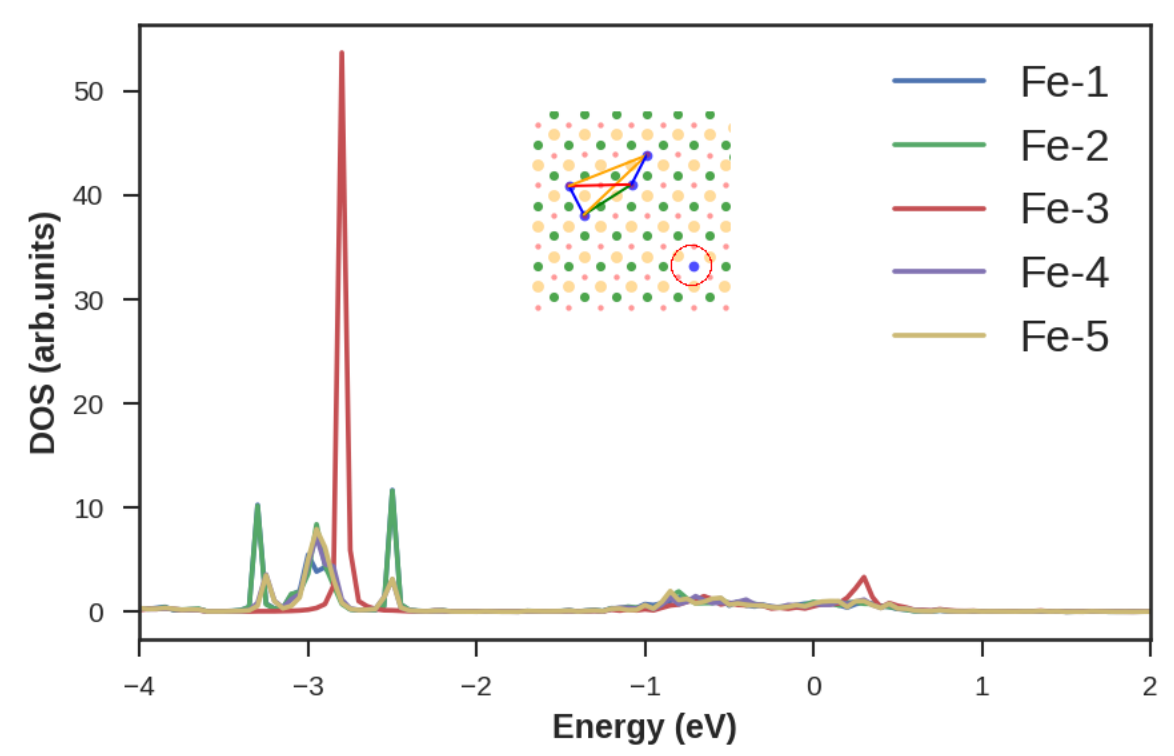

(b)

Figure 5.11: Fe orbital DOS of (a) $\mathrm{AgAl}_{0.97} \mathrm{Fe}_{0.03} \mathrm{O}_{2}$ and (b) $\mathrm{AgAl}_{0.95} \mathrm{Fe}_{0.05} \mathrm{O}_{2}$ structures. The atom corresponding to the tallest Fe peak is circled in the inset 


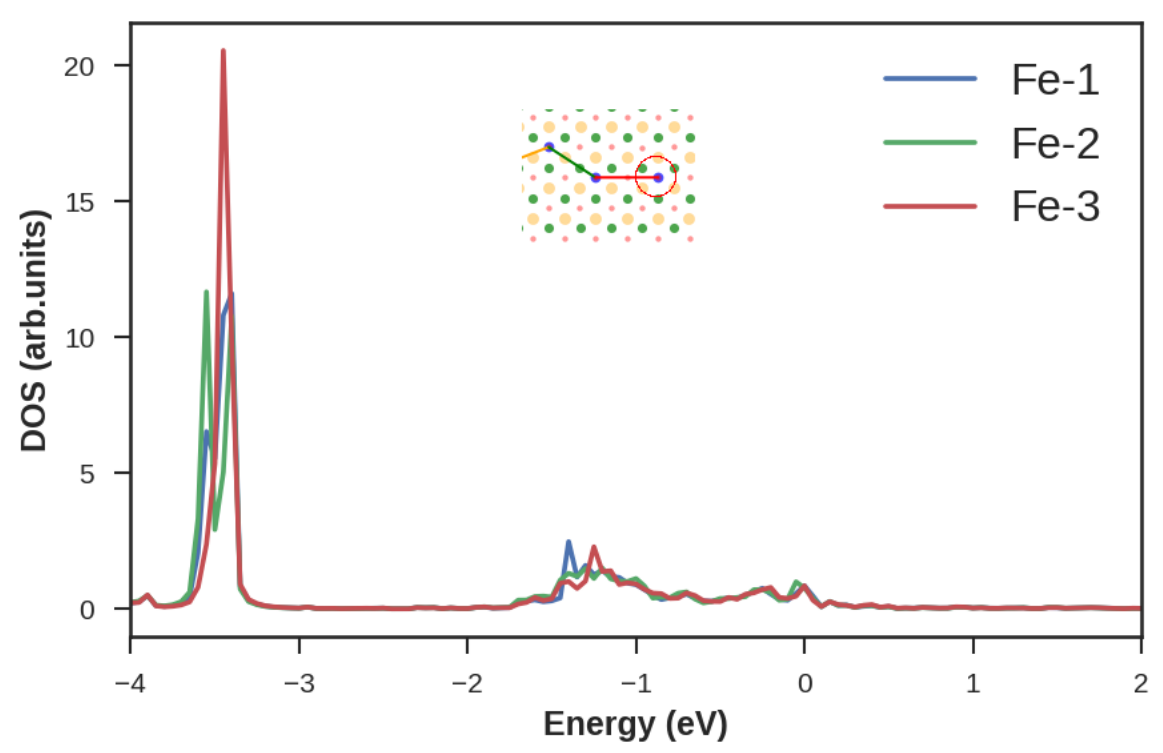

(a)

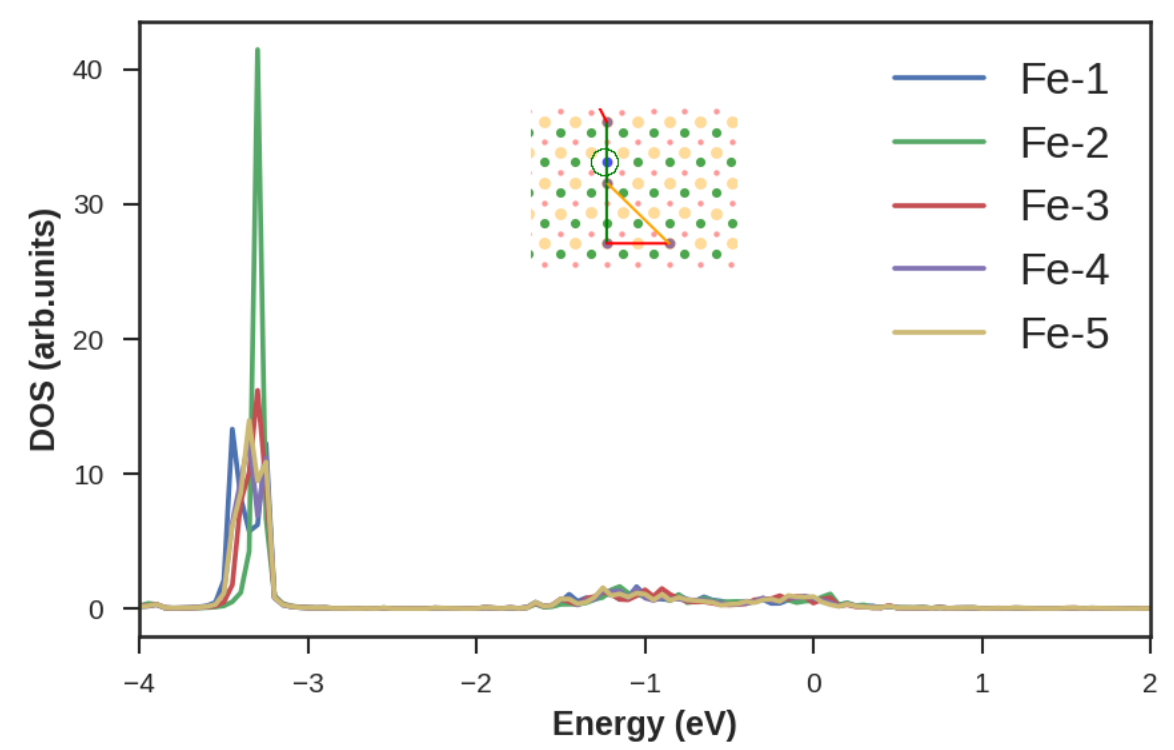

(b)

Figure 5.12: Fe orbital DOS of (a) $\mathrm{AgGa}_{0.97} \mathrm{Fe}_{0.03} \mathrm{O}_{2}$ and (b) $\mathrm{AgGa}_{0.95} \mathrm{Fe}_{0.05} \mathrm{O}_{2}$ structures. The atom corresponding to the tallest Fe peak is circled in the inset 
The energy range between $-2.75 \mathrm{eV}$ and $-1.75 \mathrm{eV}$ corresponds to the conduction band edge of $\mathrm{AgAlO}_{2}$. The states in this region are composed of hybridized O-s, Al-s, Ag-d and Ag-s orbitals (Figure 5.8 (c)). Not much hybridization is observed in the region between $-1.75 \mathrm{eV}$ and $-0.75 \mathrm{eV}$, where the states are mainly composed of O-p, Ag-s, O-s and Ag-d orbitals. The energy region corresponding to optical transitions is around $-0.3 \mathrm{eV}$ (because the valence band edge is around $-3.6 \mathrm{eV}$ and the direct gap at M point is $3.3 \mathrm{eV}$ ). The states in this region are predominantly composed of Ag-p, O-p and Ag-s states.

In $\mathrm{AgGaO}_{2}$, states close to the conduction band edge $(-3.1 \mathrm{eV}$ to $-2.75 \mathrm{eV})$ are composed of O-s, Ga-s, O-p, Ag-s and Ag-d hybridized states (Figure 5.9 (c)). As the energy increases beyond $-2.75 \mathrm{eV}$, this hybridization becomes weak and states in the region between, $-2.75 \mathrm{eV}$ to $-1.25 \mathrm{eV}$ are primarily composed of $\mathrm{O}-\mathrm{p}$ and $\mathrm{Ag}$-s states, while smaller contributions from $\mathrm{O}-\mathrm{s}$, Ga-s and Ag-d states are also visible. Similar to $\mathrm{AgAlO}_{2}$, the electron states to which the optical transitions take place are composed of Ag-p, O-p and Ag-s states. Of note is the fact that the conduction band regions of both $\mathrm{AgAl}_{1-x} \mathrm{Fe}_{x} \mathrm{O}_{2}$ and $\mathrm{AgGa}_{1-x} \mathrm{Fe}_{x} \mathrm{O}_{2}$ are not significantly disturbed due to Fe, as majority of the Fe states are in the valence band region (Figures 5.8 (d) and $5.9(\mathrm{~d}))$

To find out the effect of Fe on the electronic band structure, we used Quantum Espresso DFT package, ${ }^{236}$ as there are readily available computer programs to obtain the unfolded supercell band structures calculated by Quantum Espresso. Calculations on 432-atom supercells using Quantum Espresso requires large amounts 
computational resources. Therefore, we used 96-atom supercells (created by extending the 12-atom unit cell two times in $\mathrm{x}, \mathrm{y}$, and $\mathrm{z}$ directions) and considered Fe doping percentages of $4 \%$ and $8 \%$. Four percent Fe doping corresponds to replacing only one B-site atom with $\mathrm{Fe}\left(\frac{1}{24}=4.1 \%\right)$. Hence there is only one symmetry inequivalent structure. However, to achieve eight percent of Fe doping, we have to replace two B-site atoms with Fe. We used the bsym python package ${ }^{237}$ to find all the seven symmetry inequivalent structures. These seven structures were energetically relaxed using the FIREBALL code and the lowest energy one was selected for band structure calculations. As shown in Figure C.9 in in APPENDIX C, for both the delafossites, the structure with the two Fe atoms positioned as nearest neighbors were found to have the lowest energy. Details on the band structure calculations performed using Quantum Espresso are given given in APPENDIX C. Band unfolding was carried out using the BandUP package. ${ }^{238,239}$ A close inspection of these supercell band structures (Figure 5.13) reveals the presence of new states along the whole k-path considered for the band structure. In agreement with the DOS, the conduction band region is not much affected due to doping. The greatest change to the valence band edge occurs at the $\Gamma$ point, where the valence band edge has shifted by about $2 \mathrm{eV}$ and $1 \mathrm{eV}$ in $\mathrm{AgAl}_{1-x} \mathrm{Fe}_{x} \mathrm{O}_{2}$ and $\mathrm{AgGa}_{1-x} \mathrm{Fe}_{x} \mathrm{O}_{2}$ respectively, compared to the undoped structures. 

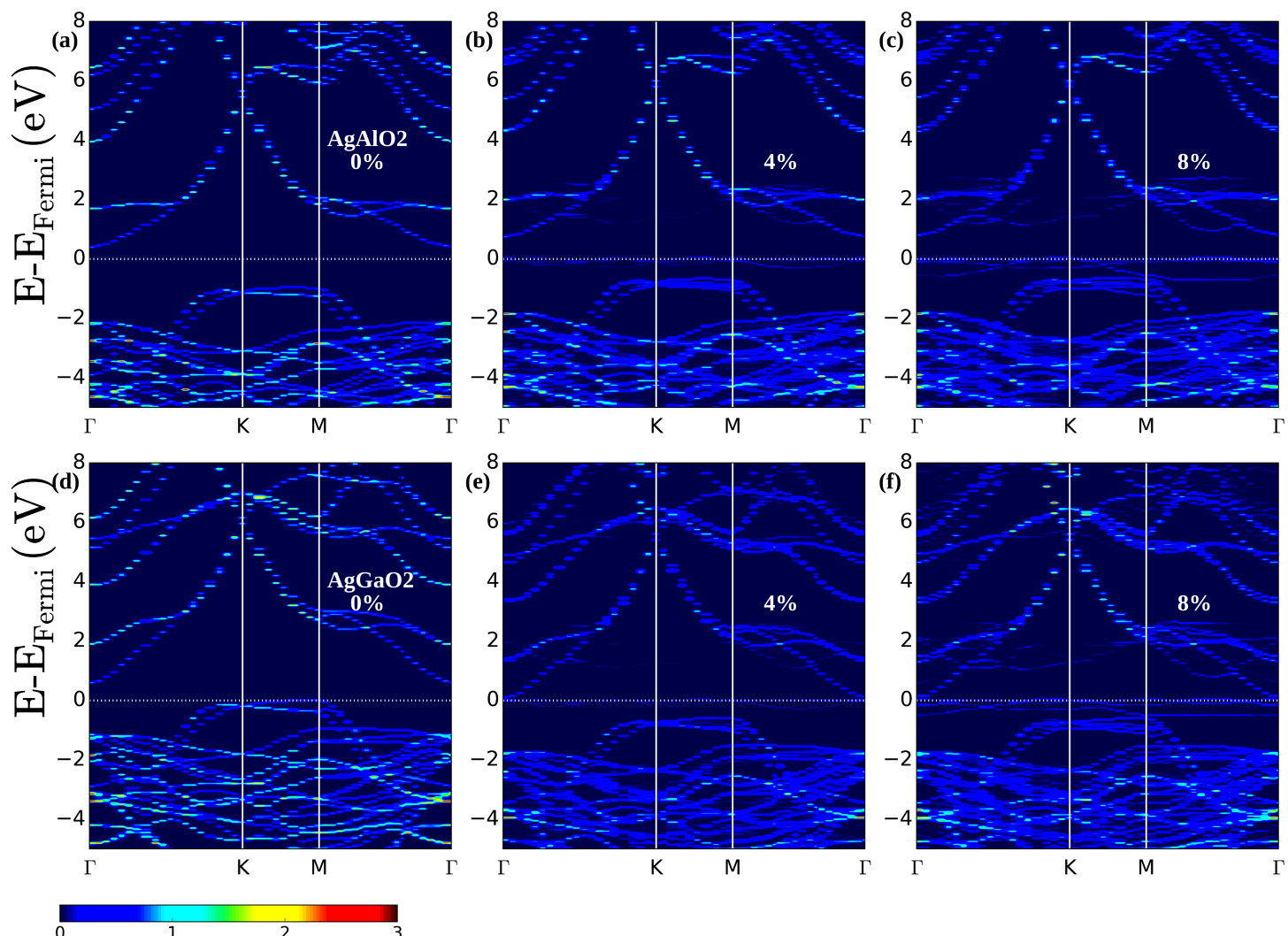

Figure 5.13: Unfolded band structures obtained for $\mathrm{AgAl}_{1-x} \mathrm{Fe}_{x} \mathrm{O}_{2}$ (a-c) and $\mathrm{AgGa}_{1-x} \mathrm{Fe}_{x} \mathrm{O}_{2}$ (d-f) by considering 96-atom supercells. The color scale represents the number of unfolded primitive cell bands crossing the primitive cell wave vector $k_{i}$ at energy $\epsilon_{i}$.

\subsubsection{Electron transitions at the $\Gamma$ point}

Laporte selection rule says that electronic transitions that take place between states having the same parity, either symmetry or anti-symmetry with respect to an inversion center are forbidden. ${ }^{240,241}$ In other words, transitions between even parity states (i.e even to even) or odd parity states (i.e odd to odd) are not allowed. For example, consider the probability for an electric dipole transition from state $\psi_{i}$ to $\psi_{f}$; 


$$
P=\int_{-\infty}^{\infty} \psi_{i} e \sum_{n} \vec{r}_{n} \psi_{i}
$$

In Equation 5.2, e $\sum_{n} \vec{r}_{n} \equiv M$ is the dipole moment operator, which is an odd function. Therefore, when $M$ acts on $\psi_{f}$, the resultant state is of the opposite parity to that of $\psi_{f}$. Thus, if $\psi_{i}$ and $\psi_{f}$ are of the same parity, $\psi_{i} e \sum_{n} \vec{r}_{n} \psi_{i}$ becomes an odd function. Because the evaluation of Equation 5.2 for an odd integrand results in zero, we find that the transitions across same parity states are not allowed.

To provide proof for the fact that Fe doping results in different parity states in valence and conduction band edges (so that the electron transitions at the $\Gamma$ point is enabled), we plotted molecular orbitals corresponding to highest occupied molecular orbital (HOMO) and lowest unoccupied molecular orbital (LUMO) eigenvalues at the $\Gamma$ point before and after Fe doping in Figures 5.14 and 5.15. These images show that valence and conduction band edges of the undoped systems consist of delocalized states which are roughly symmetric with respect to the inversion centers (A and B sites). Therefore, both HOMO and LUMO states can be considered to have even parity. Thus, the resultant probability for transitions from HOMO to LUMO becomes zero. $^{240}$ Upon Fe doping, valence band edge is primarily composed of highly localized Fe states and the resulting HOMO orbital is not symmetric with respect to the inversion centers (Figures 5.14 (b), (c) and 5.15 (b), (c)). But the conduction band edge states are still delocalized over the $\mathrm{Ag}$ and $\mathrm{O}$ states in both these delafossites. Therefore, Fe doping has changed the parity of only the conduction band edge states. In accordance with the Laporte selection rule, this change in the parity results in 
non-zero probability for electronic transitions at the $\Gamma$ point, because the parities of the states at valence and conduction band edges are now different.
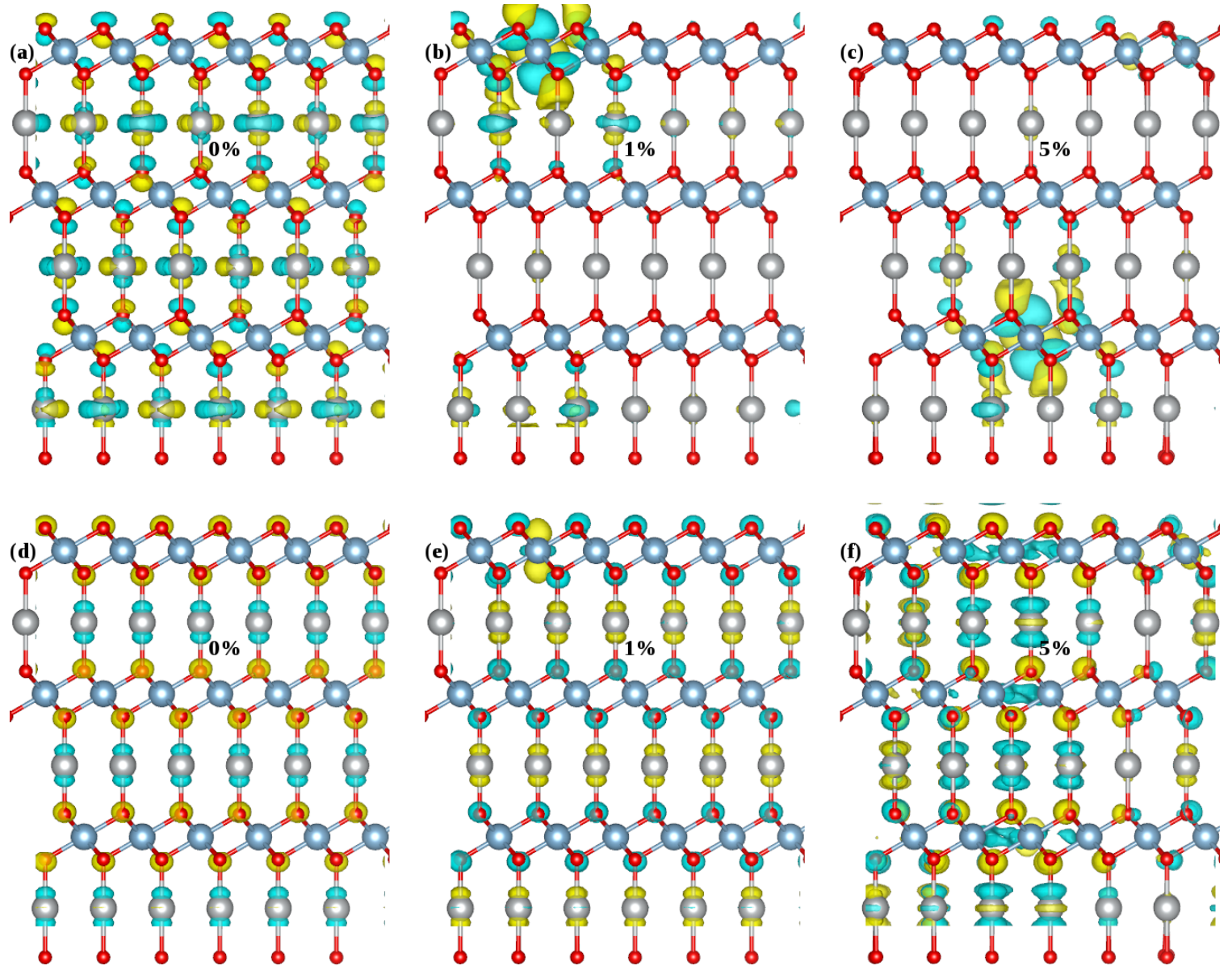

Figure 5.14: Molecular orbital plots of $\mathrm{AgAl}_{1-x} \mathrm{Fe}_{x} \mathrm{O}_{2}$. (a)-(c) correspond to the valence band edge states of $0 \%, 1 \%$ and $5 \% \mathrm{Fe}$ alloyed structures respectively. (d)-(f) correspond to the conduction band edge states of $0 \%, 1 \%$ and $5 \%$ Fe alloyed structures respectively. 

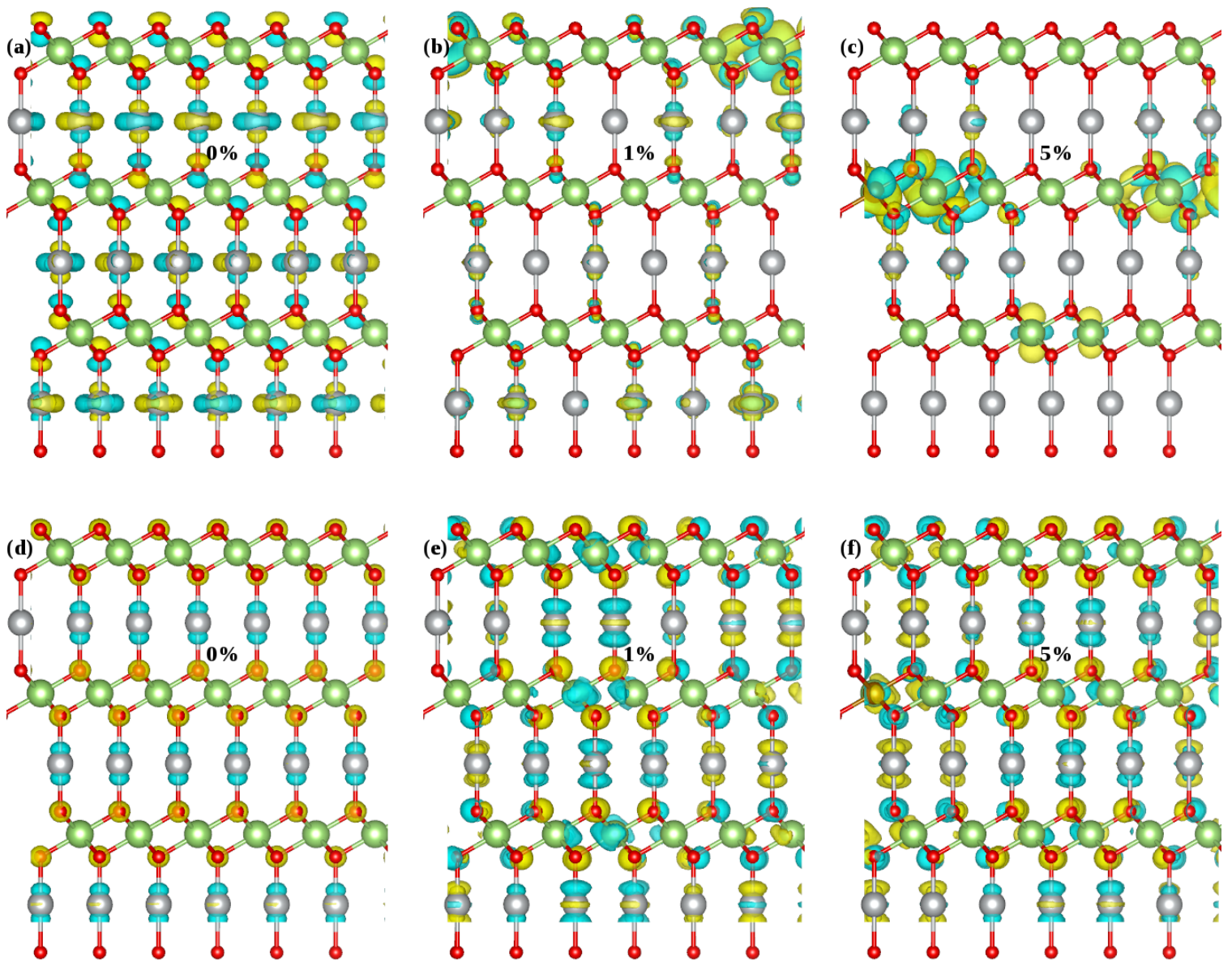

Figure 5.15: Molecular orbital plots of $\mathrm{AgGa}_{1-x} \mathrm{Fe}_{x} \mathrm{O}_{2}$. (a)-(c) correspond to the valence band edge states of $0 \%, 1 \%$ and $5 \% \mathrm{Fe}$ alloyed structures respectively. (d)-(f) correspond to the conduction band edge states of $0 \%, 1 \%$ and $5 \%$ Fe alloyed structures respectively.

\subsection{Conclusion}

We studied structural and electronic properties of two Fe doped delafossite oxides $\mathrm{AgAl}_{1-x} \mathrm{Fe}_{x} \mathrm{O}_{2}$ and $\mathrm{AgGa}_{1-x} \mathrm{Fe}_{x} \mathrm{O}_{2}$ for dopant percentages(x) 1-5\%. We found that in $\mathrm{AgAl}_{1-x} \mathrm{Fe}_{x} \mathrm{O}_{2}$, Fe atoms tend to cluster at small proximities to each other for $\mathrm{x} \leq 3 \%$. 
In contrast, two Fe atoms in low energy $\mathrm{AgGa}_{0.98} \mathrm{Fe}_{0.02} \mathrm{O}_{2}$ are separated by a second nearest neighbor distance. However, in both $\mathrm{AgAl}_{1-x} \mathrm{Fe}_{x} \mathrm{O}_{2}$ and $\mathrm{AgGa}_{1-x} \mathrm{Fe}_{x} \mathrm{O}_{2} \mathrm{Fe}$ atoms prefer to occupy the same crystallographic plane. As a result of doping, valence band edge of both systems are composed of Fe-d states. However the valence band edge of $\mathrm{AgAl}_{1-x} \mathrm{Fe}_{x} \mathrm{O}_{2}$ is slightly broadened compared to $\mathrm{AgGa}_{1-x} \mathrm{Fe}_{x} \mathrm{O}_{2}$. We also find that in $\mathrm{AgAl}_{0.98} \mathrm{Fe}_{0.02} \mathrm{O}_{2}$ the broadening in the $\mathrm{Fe}$ states is inversely proportional to the distance between Fe atoms. In both delafossites for $\mathrm{x} \geq 3 \%$, majority of the Fe density is concentrated at the relatively isolated Fe atom. This kind of relationships between structural and electronic properties are useful for band gap engineering to optimize light absorption in delafossites for photocatalytic reactions. $\mathrm{AgAl}_{1-x} \mathrm{Fe}_{x} \mathrm{O}_{2}$ shows a larger shift in the valence band edge toward higher energies than $\mathrm{AgGa}_{1-x} \mathrm{Fe}_{x} \mathrm{O}_{2}$ due to Fe impurities. Unfolded band structures reveal that the maximum shift in the valence band edge occurs at the $\Gamma$ point in both these Fe doped systems. Using molecular orbital plots, we confirmed that Fe doping affects the states only at the valence band edge, resulting in valence and conduction band edges having different parities. Thus, Fe doping can permit optical transitions that are forbidden in the pure $\mathrm{AgAlO}_{2}$ and $\mathrm{AgGaO}_{2}$. 


\section{CHAPTER VI}

\section{CONCLUSION}

Our goal in this thesis was to develop novel computational methods to understand and predict catalytic properties of alloyed catalysts. We demonstrated the functionality of these methods using three projects.

The first project defined the general framework to deal with a system having a large number of possible isomers. The problem at hand was to explain the reduction in the $\mathrm{CO}$ conversion percentages when the $\mathrm{CO}$ oxidation reaction takes place in the presence of $\mathrm{Ag}$ alloyed $\mathrm{Au}_{25}$ nanocatalysts. According to the mass spectra corresponding to the synthesis of $\mathrm{Ag}$ alloyed $\mathrm{Au}_{25}$, synthesis does not result in a single cluster species containing a specific number of $\mathrm{Ag}$ atoms. Instead, the precipitated nanocatalyst consists of a mixture of nanoclsuters containing different number of $\mathrm{Ag}$ atoms. Consequently, the $\mathrm{CO}$ conversion percentages are a result due to the combined effect of all these clusters that coexist in the same mixture. Therefore, any calculation performed to explain the catalytic trends should take into account these compositional complexities of the nanocatalyst mixture. The first step in our computational approach was to find a pool of low energy isomers which has high likelihood to exist in an experimental reaction mixture and unravel the structural trends of these isomers. In the case of bimetallic $\mathrm{Au}_{25}$, this is not an easy task as the possible 
number of alloyed isomers is very large. I adopted a two-step method to filter out a reliable set of isomers. In the first step 500 isomers were created in which Ag sites were chosen randomly. Also, $\mathrm{CH}_{3}$ was chosen as the ligand to speed up the calculations. These isomers were energetically relaxed using the FIREBALL DFT package. From the resulting low energy isomers I observed that in general, Ag prefer to occupy surface sites of the $\mathrm{M}_{13}$ core. However, to further confirm whether this is the correct trend, a second batch of isomers were created in which the Ag atoms were exclusively confined to the $\mathrm{M}_{13}$ core. Based on the results of these two calculations, it became clear that the $\mathrm{Ag}$ atoms had a definite preference to get substituted for $\mathrm{Au}$ atoms on the surface of $\mathrm{M}_{13}$ core. With this knowledge on the preferable Ag locations, I proceeded to conduct calculations to explain the observed catalytic trends. I decided to use the same ligand that the experimentalists used in the $\mathrm{CO}$ oxidation reactions, phenylethanethiolate $\left(\mathrm{CH}_{2} \mathrm{CH}_{2} \mathrm{Ph}\right)$. Condensed-to-atom dual descriptor $(\Delta f)$ was chosen as the parameter to estimate the catalytic activity. Positive and negative $\Delta f$ correspond to atomic sites that prefer to accept and donate electrons respectively. Dual descriptor values corresponding to outer shell sites of $\mathrm{Au}_{25}$ and $\mathrm{Ag}$ alloyed $\mathrm{Au}_{25}$ showed that the electron donating capability of outer shell sites reduced when $\mathrm{Au}_{25}$ is alloyed with Ag. Based on the experimental findings we believe that this reduction in the electron donating capability is likely to be a major reason for the reduction in the $\mathrm{CO}$ conversion percentages in the $\mathrm{Ag}$ alloyed clusters. Before using $\mathrm{Au}_{25} / \mathrm{CeO}_{2}$ nanocatalysts in $\mathrm{CO}$ oxidation reactions, scientists send a flow of $\mathrm{O}_{2}$ gas through the nanocatalyst $\left(\mathrm{O}_{2}\right.$ pre-treatment). This pre-treatement has been observed to increase 
the $\mathrm{CO}$ conversion in $\mathrm{Au}_{25}$ based nanocatalysts in previous experiments. The authors of these studies believe that $\mathrm{O}_{2}$ pre-treatment creates a large number of active oxygen species at the interface between $\mathrm{Au}_{25}$ and $\mathrm{CeO}_{2}$. Such active oxygen can help $\mathrm{CO}$ oxidation take place smoothly. The generation of active oxygen species like superoxide and peroxide require $\mathrm{O}_{2}$ acquiring electrons. Based on another previous study, it is argued that $\mathrm{Au}_{25}$, which is a negatively charged cluster can readily donate an electron to $\mathrm{O}_{2}$. Therefore the reduction in the electron donating capability of the Ag alloyed $\mathrm{Au}_{25}$ clusters is highly likely to be a main contributing factor for the observed reduction in $\mathrm{CO}$ conversion percentages. Comparison of the $\mathrm{CO}$ conversion percentages corresponding to $\mathrm{Au}_{25}$ containing different number of $\mathrm{Ag}$ dopants showed that the catalytic activity of heavily doped $\mathrm{Au}_{25}$ is less than that of moderately doped $\mathrm{Au}_{25}$. According to dual descriptor values, outer shell sites of heavily doped $\mathrm{Au}_{25}$ prefer to attract electrons. As a result, the amount of active oxygen species generated in the case of heavily doped $\mathrm{Au}_{25}$ would be much less than in the case of moderately doped $\mathrm{Au}_{25}$.

Being able to explain experimental trends is essential for the advancement of research on catalysis. However, what is more helpful is the ability to predict catalytic properties in advance, allowing scientists to find the composition of catalytic systems having desired properties for given applications. Such prior knowledge immensely reduces the time and effort spent on research and development in search of optimal catalytic products. In our second project, we developed a machine learning model to predict adsorption energies of thiolated nanoclusters. The features of our model 
are based on geometric properties of non-relaxed adsorbate-free nanoclusters. The use of such features enables one to create time and resource efficient models requiring minimum information as input. Training our machine learning model consists of two phases. In the first phase, all the features are fed into a random forest algorithm to find the feature importance and subsequently the optimal features that can produce the best prediction accuracy. In the second phase, the best features are used to engineer additional features that possibly have non-linear relationships with adsorption energies. For the $\mathrm{Au}_{25}$ nanocluster, we found that the number of $\mathrm{Ag}$ atoms was the most important feature while most of the other top features are based on the clustering of the $\mathrm{Ag}$ atoms. Also among the top ten features is $\mathrm{HCH} 1$, the number of H-C-H fractions closest to the adsorption site. This indicates that the orientation of the ligands also plays a significant role in determining the adsorption energies in $\mathrm{Au}_{25}$. The feature-feature correlation maps that we generated can be utilized by experimentalists in the design of novel materials. For example, the high linear correlation between the adsorption energies and the features based on the clustering of the Ag atoms indicated that the adsorption energies are strongly affected by the spread of the Ag dopants with respect to their centroids. In other words, the smaller the $\mathrm{Ag}-\mathrm{Ag}$ distance, the more stronger is the adsorption between $\mathrm{CO}$ and the adsorption site. We also noticed that our model performance is not very good in predicting positive adsorption energies. We attribute this poor performance to the lack of training samples corresponding to positive adsorption energies and the presence of multiple isomers having similar adsorption energies. In a future project we plan to address 
this issue. To show that our features can be used to predict adsorption energies of nanocusters of any size and shape, we also predicted $\mathrm{CO}$ adsorption energies of $\mathrm{Au}_{36}$ and $\mathrm{Au}_{133}$ nanoclusters.

$\mathrm{Au}_{36}$ is less spherical than $\mathrm{Au}_{25}$ and has planar facets composed of $\mathrm{Au}$ atoms. Interestingly, we found that features based on the ligand environment have a decisive impact on the prediction accuracy. We believe that this is due to the fact that ligands attached to planar surfaces cause more steric hindrance to approaching molecules than the ligands in a spherical cluster. To test this hypothesis we calculated the average $\mathrm{H}-\mathrm{C}$ bonds closest to the adsorption sites in $\mathrm{Au}_{25}, \mathrm{Au}_{36}$ and $\mathrm{Au}_{133}$ nanoclusters. We found that this number is larger for $\mathrm{Au}_{36}$ clusters compared to both $\mathrm{Au}_{25}$ and $\mathrm{Au}_{133}$ which are both more spherical than $\mathrm{Au}_{36}$.

$\mathrm{Au}_{133}$ is the largest thiolate-protected nanocluster of which the crystal structure has been resolved crystallographically. The prediction accuracies of $\mathrm{Au}_{133}$ are similar to those of $\mathrm{Au}_{25}$. Predicting adsorption energies of $\mathrm{Au}_{133}$ required more features than those required in the cases of $\mathrm{Au}_{25}$ and $\mathrm{Au}_{36}$. This increase could be related to the larger size of $\mathrm{Au}_{133}$ compared to the other clusters. When the cluster size increases so does the number of available Ag dopant locations. Thus more in formation is required by the machine learning algorithm to uniquely identify a given alloyed cluster.

Overall, our machine learning model can be considered as an efficient tool to filter out a candidate set of isomers using minimum amount of information on structural properties. As the features are based on non-relaxed isomers, no DFT cal- 
culations are required in the testing phase, which conveniently reduces the prediction time. Even though the functionality is demonstrated using adsorption energy prediction, our model can be easily extended to predict any other materials property. What would be remarkable is a predictive model that can provide users with an equation relating the adsorption energies and the features. Such functions allow researchers to readily determine the composition of a nanocluster having a particular adsorption energy. Even though I tried to achieve this goal using a kernel ridge regression based model, the resultant prediction accuracies were not satisfactory.

Delafossites have been studied since 1940's and become popular in 1970's as transparent conductive oxides. However, research has shown that these systems also have potential applications in the fields of thermoelectrics, superconductors and batteries. It was recently shown that Fe doping reduces the optical gaps and consequently the photocatalytic activity of $\mathrm{CuGaO}_{2}$ and $\mathrm{NaInO}_{2}$ delafossites. Even though the silver family of delafossites have also been shown to posses photocatalytic properties, it was surprising to find that no experimental or theoretical work has been carried out on the doping effects of these systems. Hence we decided to explore the Fe doping effects of two $\mathrm{Ag}$ delafossite oxides, $\mathrm{AgAlO}_{2}$ and $\mathrm{AgGaO}_{2}$. As it was the case with alloyed nanoclusters, Fe doping results in a large number of possible crystal structures for these delafossites. After energetically relaxing a set of randomly $\mathrm{Fe}$ doped delafossite structures, we studied the trends in the Fe dopant locations using two parameters. The clustering factor is a measure of how closely the Fe atoms are located with respect to each other. Small clustering factors correspond to closely 
clustered Fe atoms. We noticed that when Fe percentage is less than or equal to 3, Fe atoms in $\mathrm{AgAlO}_{2}$ prefer to get substituted for nearest neighbor $\mathrm{Al}$ atoms. This trend is not observed in $\mathrm{AgGa}_{1-x} \mathrm{Fe}_{x} \mathrm{O}_{2}$. We suspect that this discrepancy may be related to the smaller atomic radius of $\mathrm{Al}$ compared to Ga. However, further calculations are needed to unravel the underlying reasons. The other structural parameter checks whether there is a preference for majority of the Fe dopants to occupy a single B-site plane. Interestingly, we find that in both $\mathrm{AgAlO}_{2}$ and $\mathrm{AgGaO}_{2}$, Fe dopants do prefer to be on the same plane to minimize the structural energy. The changes in the electronic structure due to Fe in both these systems are similar. The main effect due to Fe is the shift in the valence band edge toward higher energies. The Fermi level is composed mainly of Fe-d states. No significant changes in the conduction band region are observed due to $\mathrm{Fe}$. We also noticed that $\mathrm{Fe}$ states are localized at the $\mathrm{Fe}$ atom which is relatively isolated from the rest. However Fe states in $\mathrm{AgAl}_{1-x} \mathrm{Fe}_{x} \mathrm{O}_{2}$ are observed to be more broadened than the Fe states in $\mathrm{AgGa}_{1-x} \mathrm{Fe}_{x} \mathrm{O}_{2}$. The unfolded band structures obtained using Quantum Espresso showed the appearance of new states near the Fermi level which confirmed the DOS results. We also see that the these new states are more broadened near $\Gamma$ and $\mathrm{M}$ points compared to the other states. As the optical gaps are likely to occur at the M point, the above mentioned changes in the electronic structure have the effect of reducing the optical gaps. Therefore, Fe doping can be considered as an effective method to engineer band gaps for photocatalytic applications. We suggest further calculations to study possible effects in the electronic structure due to magnetic nature of Fe dopants. Our belief is that 
for low amounts of Fe doping magnetic effects are negligible as it was shown for $\mathrm{Fe}$ doped $\mathrm{CuGaO}_{2} \cdot{ }^{230}$ 


\section{BIBLIOGRAPHY}

[1] Breakthrough catalyst. Research \& Development (2005). [Online; posted August 2005].

[2] Catalyst Market Size Worth \$34.06 Billion By 2025 | CAGR: 4.5\% (2019). [Online; posted February 2019].

[3] Mingos, D. M. P. Historical Introduction to Gold Colloids, Clusters and Nanoparticles, 1-47 (Springer International Publishing, Cham, 2014).

[4] Everett, D. H. Manual of symbols and terminology for physicochemical quantities and units, appendix II: Definitions, terminology and symbols in colloid and surface chemistry. Pure and Applied Chemistry 31, 577-638 (1972).

[5] Higham, T. et al. New perspectives on the varna cemetery (bulgaria) - ams dates and social implications. Antiquity 81, 640-654 (2007).

[6] Krauß, R. et al. Chronology and development of the chalcolithic necropolis of varna i 44, 282-305 (2017).

[7] Oppenheim, A. \& Goelet, O. The Art and Culture of Ancient Egypt: Studies in Honor of Dorothea Arnold (Bulletin of the Egyptological Seminar of New York) (English, French and German Edition) (Egyptological Seminar of New York, 2015).

[8] Faraday, M. The Bakerian Lecture: Experimental Relations of Gold (and Other Metals) to Light. Philosophical Transactions of the Royal Society of London Series I 147, 145-181 (1857).

[9] Walter, S. Über die optischen eigenschaften kolloidaler goldlösungen. Annalen der Physik 331, 329-371 (1908).

[10] Gustav, M. Beiträge zur optik trüber medien, speziell kolloidaler metallösungen. Annalen der Physik 330, 377-445 (1908). 
[11] Haruta, M., Kobayashi, T., Sano, H. \& Yamada, N. Novel gold catalysts for the oxidation of carbon monoxide at a temperature far below $0{ }^{\circ} \mathrm{c}$. Chemistry Letters 16, 405-408 (1987).

[12] Naldini, L., Cariati, F., Simonetta, G. \& Malatesta, L. Gold-tertiary phosphine derivatives with intermetallic bonds. Chem. Commun. (London) 647-648 (1966).

[13] Vollenbroek, F. A., Bosman, W. P., Bour, J. J., Noordik, J. H. \& Beurskens, P. T. Reactions of gold-phosphine cluster compounds. preparation and x-ray structure determination of octakis(triphenylphosphine)octa-gold bis(hexafluorophosphate). J. Chem. Soc., Chem. Commun. 387-388 (1979).

[14] Vollenbroek, F. A., Bour, J. J. \& van der Veden, J. W. A. Gold-phosphine cluster compounds: The reactions of $\left[\mathrm{Au}_{9} \mathrm{~L}_{8}\right]^{3+}\left(\mathrm{L}=\mathrm{PPh}_{3}\right)$ with $\mathrm{L}, \mathrm{SCN}^{-}$and $\mathrm{Cl}^{-}$to $\left[\mathrm{Au}_{8} \mathrm{~L}_{8}\right]^{2+}\left(\mathrm{Au}_{11} \mathrm{~L}_{8}(\mathrm{SCN})_{2}\right]^{+}$and $\left[\mathrm{Au}_{11} \mathrm{~L}_{8} \mathrm{Cl}_{2}\right]^{+}$. Recueil des Travaux Chimiques des Pays-Bas 99, 137-141 (1980).

[15] Van der Velden, J. W. A., Bour, J. J., Bosman, W. P. \& Noordik, J. H. Synthesis and x-ray crystal structure determination of the cationic gold cluster compound $\left[\mathrm{Au}_{8}\left(\mathrm{PPh}_{3}\right)_{7}\right]\left(\mathrm{NO}_{3}\right)_{2}$. J. Chem. Soc., Chem. Commun. 1218-1219 (1981).

[16] Van der Velden, J. W. A. et al. Gold clusters containing bidentate phosphine ligands. preparation and $\mathrm{x}$-ray structure investigation of $\left[\mathrm{Au}_{5}(\mathrm{dppmH})_{3}(\mathrm{dppm})\right]\left(\mathrm{NO}_{3}\right)_{2}$ and $\left[\mathrm{Au}_{13}(\mathrm{dppmH})_{6}\right]\left(\mathrm{NO}_{3}\right)_{n}$. Recueil des Travaux Chimiques des Pays-Bas 100, 148-152 (1981).

[17] Van der Velden, J. W. A. et al. Gold clusters. tetrakis[1,3-bis(diphenylphosphino)propane]hexa dinitrate: preparation, x-ray analysis, and gold-197 Moessbauer and phosphorus31 pproton\} NMR spectra. Inorganic Chemistry 21, 4321-4324 (1982).

[18] Briant, C. E. et al. Synthesis and x-ray structural characterization of the centred icosahedral gold cluster compound $\left[\mathrm{Au}_{l 3}\left(\mathrm{PMe}_{2} \mathrm{Ph}\right)_{10} \mathrm{Cl}_{2}\right]\left(\mathrm{PF}_{6}\right)_{3}$; the realization of a theoretical prediction. J. Chem. Soc., Chem. Commun. 201-202 (1981).

[19] Hall, K. P., Theoblad, B. R. C., Gilmour, D. I., Mingos, D. M. P. \& Welch, A. J. Synthesis and structural characterization of $\left[\mathrm{Au}_{9} \mathrm{P}\left(\mathrm{p}-\mathrm{C}_{6} \mathrm{H}_{4} \mathrm{OMe}\right)_{38}\right]\left(\mathrm{BF}_{4}\right)_{3}$; a cluster with a centred crown of gold atoms. J. Chem. Soc., Chem. Commun. 528-530 (1982). 
[20] Briant, C. E., Hall, K. P. \& Mingos, D. M. P. Structural characterisation of two crystalline modifications of $\left[\mathrm{Au}_{9} \mathrm{P}\left(\mathrm{C}_{6} \mathrm{H}_{4} \mathrm{OMe}-\mathrm{p}\right)_{38}\right]\left(\mathrm{NO}_{3}\right)_{3}$ : the first example of skeletal isomerism in metal cluster chemistry. J. Chem. Soc., Chem. Commun. 290-291 (1984).

[21] Briant, C. E., Hall, K. P., Mingos, D. M. P. \& Wheeler, A. C. Synthesis and structural characterisation of hexakis(triphenyl phosphine)hexagold $(2+)$ nitrate, $\left[\mathrm{Au}_{6}\left(\mathrm{PPh}_{3}\right)_{6}\right]\left[\mathrm{NO}_{3}\right]_{2}$, and related clusters with edgesharing bitetrahedral geometries. J. Chem. Soc., Dalton Trans. 687-692 (1986).

[22] Brust, M., Walker, M., Bethell, D., Schiffrin, D. J. \& Whyman, R. Synthesis of thiol-derivatised gold nanoparticles in a two-phase liquid-liquid system. $J$. Chem. Soc., Chem. Commun. 801-802 (1994).

[23] Whetten, R. L. et al. Nanocrystal gold molecules. Advanced Materials 8, 428433 (1996).

[24] Schaaff, T. G. et al. Isolation of smaller nanocrystal au molecules: robust quantum effects in optical spectra. The Journal of Physical Chemistry B 101, 7885-7891 (1997).

[25] Häkkinen, H., Barnett, R. N. \& Landman, U. Electronic structure of passivated $\mathrm{au}_{38}\left(\mathrm{sch}_{3}\right)_{24}$ nanocrystal. Phys. Rev. Lett. 82, 3264-3267 (1999).

[26] Häkkinen, H., Walter, M. \& Grönbeck, H. Divide and protect: capping gold nanoclusters with molecular gold-thiolate rings. The Journal of Physical Chemistry B 110, 9927-9931 (2006).

[27] Jadzinsky, P. D., Calero, G., Ackerson, C. J., Bushnell, D. A. \& Kornberg, R. D. Structure of a thiol monolayer-protected gold nanoparticle at 1.1 a resolution. Science (New York, N.Y.) 318, 430-3 (2007).

[28] Zhu, M., Aikens, C. M., Hollander, F. J., Schatz, G. C. \& Jin, R. Correlating the crystal structure of a thiol-protected au25 cluster and optical properties. Journal of the American Chemical Society 130, 5883-5885 (2008).

[29] Jin, R. et al. Size focusing: A methodology for synthesizing atomically precise gold nanoclusters (2010).

[30] Zeng, C., Chen, Y., Das, A. \& Jin, R. Transformation chemistry of gold nanoclusters: From one stable size to another. The Journal of Physical Chemistry Letters 6, 2976-2986 (2015). 
[31] Fields-Zinna, C. A., Crowe, M. C., Dass, A., Weaver, J. E. F. \& Murray, R. W. Mass spectrometry of small bimetal monolayer-protected clusters. Langmuir 25, 7704-7710 (2009).

[32] Negishi, Y. et al. Isolation, structure, and stability of a dodecanethiolateprotected $\mathrm{Pd}_{1} \mathrm{Au}_{24}$ cluster. Physical Chemistry Chemical Physics 12, 6219 (2010).

[33] Negishi, Y., Iwai, T. \& Ide, M. Continuous modulation of electronic structure of stable thiolate-protected $\mathrm{Au}_{25}$ cluster by $\mathrm{Ag}$ doping. Chemical Communications 46, 4713-4715 (2010).

[34] Doping 25-atom and 38-atom gold nanoclusters with palladium. Acta PhysicoChimica Sinica 27 (2011).

[35] Kumara, C. \& Dass, A. (AuAg) ${ }_{144}(\mathrm{SR})_{60}$ alloy nanomolecules. Nanoscale 3, 3064-7 (2011).

[36] Negishi, Y., Igarashi, K., Munakata, K., Ohgake, W. \& Nobusada, K. Palladium doping of magic gold cluster $\mathrm{Au}_{38}\left(\mathrm{SC}_{2} \mathrm{H}_{4} \mathrm{ph}\right)_{24}$ : formation of $\mathrm{Pd}_{2} \mathrm{Au}_{36}\left(\mathrm{SC}_{2} \mathrm{H}_{4} \mathrm{Ph}\right)_{24}$ with higher stability than $\mathrm{Au}_{38}\left(\mathrm{SC}_{2} \mathrm{H}_{4} \mathrm{Ph}\right)_{24}$. Chem. Commun. 48, 660-662 (2012).

[37] Qian, H. et al. Monoplatinum doping of gold nanoclusters and catalytic application. Journal of the American Chemical Society 134, 16159-16162 (2012).

[38] Eric, G., Huifeng, Q. \& Rongchao, J. Atomic-Level alloying and De-alloying in doped gold nanoparticles. Chemistry - A European Journal 19, 4238-4243 (2013).

[39] Kothalawala, N., Kumara, C., Ferrando, R. \& Dass, A. $\operatorname{Au}_{144-x} \operatorname{Pd}_{x}(\mathrm{SR})_{60}$ nanomolecules. Chem. Commun. 49, 10850-10852 (2013).

[40] Dharmaratne, A. C. \& Dass, A. $\mathrm{Au}_{144-x} \mathrm{Cu}_{x}\left(\mathrm{SC}_{6} \mathrm{H}_{13}\right)_{60}$ nanomolecules: effect of cu incorporation on composition and plasmon-like peak emergence in optical spectra. Chem. Commun. 50, 1722-1724 (2014).

[41] Kumara, C., Aikens, C. M. \& Dass, A. X-ray crystal structure and theoretical analysis of $\mathrm{Au}_{25-\mathrm{x}} \mathrm{Ag}_{\mathrm{x}}\left(\mathrm{SCH}_{2} \mathrm{CH}_{2} \mathrm{Ph}\right)_{18}^{-}$alloy. The Journal of Physical Chemistry Letters 5, 461-466 (2014).

[42] Yao, C. et al. Mono-cadmium vs mono-mercury doping of $a_{25}$ nanoclusters. Journal of the American Chemical Society 137, 15350-3 (2015). 
[43] Li, Q. et al. Heavily doped $\mathrm{Au}_{25-\mathrm{x}} \mathrm{Ag}_{\mathrm{x}}\left(\mathrm{SC}_{6} \mathrm{H}_{11}\right)_{18}{ }^{-}$nanoclusters: silver goes from the core to the surface. Chemical Communications 52, 5194-5197 (2016).

[44] Marquardt, M. A., Ashmore, N. A. \& Cann, D. P. Crystal chemistry and electrical properties of the delafossite structure. Thin Solid Films 496, 146156 (2006).

[45] Shannon, R. D., Rogers, D. B. \& Prewitt, C. T. Chemistry of noble metal oxides. I. syntheses and properties of $\mathrm{ABO} 2$ delafossite compounds. Inorganic Chemistry 10, 713-718 (1971).

[46] Shannon, R. D., Prewitt, C. T. \& Rogers, D. B. Chemistry of noble metal oxides. II. crystal structures of platinum cobalt dioxide, palladium cobalt dioxide, coppper iron dioxide, and silver iron dioxide. Inorganic Chemistry 10, 719-723 (1971).

[47] Shannon, R. D., Rogers, D. B., Prewitt, C. T. \& Gillson, J. L. Chemistry of noble metal oxides. III. electrical transport properties and crystal chemistry of abo2 compounds with the delafossite structure. Inorganic Chemistry 10, 723-727 (1971).

[48] Carcia, P. F., Shannon, R. D., Bierstedt, P. E. \& Flippen, R. B. O 2 electrocatalysis on thin film metallic oxide electrodes with the delafossite structure. Journal of The Electrochemical Society 127, 1974-1978 (1980).

[49] Benko, F. \& Koffyberg, F. Opto-electronic properties of $\mathrm{CuAlO}_{2}$. Journal of Physics and Chemistry of Solids 45, 57 - 59 (1984).

[50] Monnier, J., Hanrahan, M. \& Apai, G. A study of the catalytically active copper species in the synthesis of methanol over $\mathrm{Cu}-\mathrm{Cr}$ oxide. Journal of Catalysis 92, $119-126$ (1985).

[51] Doumerc, J.-P., Wichainchai, A., Ammar, A., Pouchard, M. \& Hagenmuller, P. On magnetic properties of some oxides with delafossite-type structure. Materials Research Bulletin 21, 745 - 752 (1986).

[52] Otabe, T., Ueda, K., Kudoh, A., Hosono, H. \& Kawazoe, H. n-type electrical conduction in transparent thin films of delafossite-type AgInO2. Applied Physics Letters 72, 1036-1038 (1998).

[53] Isawa, K. et al. Thermoelectric power of delafossite-derived compounds, $\mathrm{RCuO}_{2+\delta}$ (R = Y, La, Pr, Nd, Sm, and Eu). Phys. Rev. B 57, 7950-7954 (1998). 
[54] Gong, H., Wang, Y. \& Luo, Y. Nanocrystalline p-type transparent Cu-Al$\mathrm{O}$ semiconductor prepared by chemical-vapor deposition with $\mathrm{Cu}(\mathrm{acac})_{2}$ and $\mathrm{Al}(\mathrm{acac})_{3}$ precursors. Applied Physics Letters 76, 3959-3961 (2000).

[55] Yanagi, H. et al. Electronic structure and optoelectronic properties of transparent p-type conducting $\mathrm{CuAlO}_{2}$. Journal of Applied Physics 88, 4159-4163 (2000).

[56] Ueda, K. et al. Epitaxial growth of transparent p-type conducting CuGaO2 thin films on sapphire (001) substrates by pulsed laser deposition. Journal of Applied Physics 89, 1790-1793 (2001).

[57] Yanagi, H., Hase, T., Ibuki, S., Ueda, K. \& Hosono, H. Bipolarity in electrical conduction of transparent oxide semiconductor $\mathrm{CuInO} 2$ with delafossite structure. Applied Physics Letters 78, 1583-1585 (2001).

[58] Yanagi, H. et al. Fabrication of all oxide transparent p-n homojunction using bipolar CuInO2 semiconducting oxide with delafossite structure. Solid State Communications 121, 15-17 (2001).

[59] Tate, J. et al. p-type oxides for use in transparent diodes. Thin Solid Films 411, 119-124 (2002).

[60] Ataoui, K. E. et al. $\mathrm{M}\{\ddot{0}\}$ ssbauer study and magnetic properties of $\mathrm{CuFe} 1-\mathrm{xGaxO2}$. Journal of Alloys and Compounds 368, 79-83 (2004).

[61] Okuda, T., Jufuku, N., Hidaka, S. \& Terada, N. Magnetic, transport, and thermoelectric properties of the delafossite oxides $\mathrm{CuCr}_{1-x} \mathrm{Mg}_{x} \mathrm{O}_{2}(0 \leq \mathrm{x} \leq$ 0.04). Physical Review B 72, 144403 (2005).

[62] Vanaja, K. A., Ajimsha, R. S., Asha, A. S. \& Jayaraj, M. K. p-type electrical conduction in $\alpha-\mathrm{AgGaO}_{2}$ delafossite thin films. Applied Physics Letters 88, 212103 (2006).

[63] Pellicer-Porres, J. et al. On the band gap of $\mathrm{CuAlO}_{2}$ delafossite. Applied Physics Letters 88, 181904 (2006).

[64] Snure, M. \& Tiwari, A. $\mathrm{CuBO}_{2}$ : A p-type transparent oxide. Applied Physics Letters 91, 092123 (2007).

[65] Sheets, W. C. et al. Silver delafossite oxides. Inorganic Chemistry 47, 26962705 (2008). 
[66] Dong, H. et al. Visible light-induced photocatalytic activity of delafossite $\mathrm{AgMO}_{2}(\mathrm{M}=\mathrm{Al}, \mathrm{Ga}, \mathrm{In})$ prepared via a hydrothermal method. Applied Catalysis B: Environmental 89, 551-556 (2009).

[67] Guilmeau, E., Maignan, A. \& Martin, C. Thermoelectric oxides: Effect of doping in delafossites and zinc oxide. Journal of Electronic Materials 38, 11041108 (2009).

[68] Ketir, W., Bouguelia, A. \& Trari, M. $\mathrm{NO}_{3}$-removal with a new delafossite $\mathrm{CuCrO}_{2}$ photocatalyst. Desalination 244, 144-152 (2009).

[69] Huda, M. N., Yan, Y., Walsh, A., Wei, S.-H. \& Al-Jassim, M. M. Group-IIIA versus IIIB delafossites: Electronic structure study. Physical Review B 80, 035205 (2009).

[70] Maignan, A. et al. Electronic structure and thermoelectric properties $\mathrm{CuRh}_{1-x} \mathrm{Mg}_{x} \mathrm{O}_{2}$. Physical Review B 80, 115103 (2009).

[71] Singh, M., Mehta, B. R., Varandani, D. \& Singh, V. N. Electrical and optical properties of sn doped $\mathrm{CuInO}_{2}$ thin films: Conducting atomic force microscopy and spectroscopic ellipsometry studies. Journal of Applied Physics 106, 053709 (2009).

[72] Bensaid, S. \& Russo, N. Low temperature DPF regeneration by delafossite catalysts. Catalysis Today 176, 417-423 (2011).

[73] Poopanya, P., Yangthaisong, A., Rattanapun, C. \& Wichainchai, A. Theoretical study of electronic structure and thermoelectric properties of doped $\mathrm{CuAlO}_{2}$. Journal of Electronic Materials 40, 987-991 (2011).

[74] Scanlon, D. O. \& Watson, G. W. Understanding the p-type defect chemistry of $\mathrm{CuCrO}_{2}$. Journal of Materials Chemistry 21, 3655-3663 (2011).

[75] Ruttanapun, C. et al. Thermoelectric properties of $\mathrm{Cu}_{1-x} \mathrm{Pt}_{x} \mathrm{FeO}_{2}(0.0 \leq \mathrm{x} \leq$ 0.05) delafossite-type transition oxide. Journal of Alloys and Compounds 509, 4588-4594 (2011).

[76] Nakanishi, A. \& Katayama-Yoshida, H. Computational materials design for superconductivity in hole-doped delafossite $\mathrm{CuAlO}_{2}$ : Transparent superconductors. Solid State Communications 152, 24-27 (2012). 
[77] Zhao, Y., Natsume, Y., Sawaguchi, N. \& Sasaki, M. Synthesis and optoelectrical properties of $\mathrm{ABO}_{2}(\mathrm{~A}=\mathrm{Li}, \mathrm{Na} ; \mathrm{B}=\mathrm{Y}, \mathrm{Yb})$. IOP Conference Series: Materials Science and Engineering 18, 092066 (2011).

[78] Yu, M., Natu, G., Ji, Z. \& Wu, Y. p-type dye-sensitized solar cells based on delafossite $\mathrm{CuGaO}_{2}$ nanoplates with saturation photovoltages exceeding 460 mV. The Journal of Physical Chemistry Letters 3, 1074-1078 (2012).

[79] Ruttanapun, C. et al. Electrical and optical properties of p-type $\mathrm{CuFe}_{1-\mathrm{x}} \mathrm{Sn}_{\mathrm{x}} \mathrm{O}_{2}$ $(\mathrm{x}=0.03,0.05)$ delafossite-oxide. Journal of Applied Physics 113, 023103 (2013).

[80] Kumar, M., Zhao, H. \& Persson, C. Study of band-structure, optical properties and native defects in $\mathrm{A}^{I} \mathrm{~B}^{I I I} \mathrm{O}_{2}\left(\mathrm{~A}^{I}=\mathrm{Cu}\right.$ or $\mathrm{Ag}, \mathrm{B}^{I I I}=\mathrm{Al}$, Ga or In) delafossites. Semiconductor Science and Technology 28, 065003 (2013).

[81] Ruttanapun, C. Optical and electronic properties of delafossite $\mathrm{CuBO}_{2}$ p-type transparent conducting oxide. Journal of Applied Physics 114, 113108 (2013).

[82] Han, M. J. et al. Electronic transition and electrical transport properties of delafossite $\mathrm{CuCr}_{1-x} \mathrm{Mg}_{x} \mathrm{O}_{2}(0 \leq x \leq 12 \%)$ films prepared by the sol-gel method: A composition dependence study. Journal of Applied Physics 114, 163526 (2013).

[83] Haycock, B. J., Rice, M. K. \& Lewis, J. P. High-throughput calculations of alloyed delafossite materials: Application to $\mathrm{CuGa}_{1-x} \mathrm{Fe}_{x} \mathrm{O}_{2}$. Computational Materials Science 86, 155 - 164 (2014).

[84] Iozzi, M. F., Vajeeston, P., Vidya, R., Ravindran, P. \& Fjellvåg, H. Structural and electronic properties of transparent conducting delafossite: a comparison between the $\mathrm{AgBO}_{2}$ and $\mathrm{CuBO}_{2}$ families $(\mathrm{B}=\mathrm{Al}, \mathrm{Ga}$, In and $\mathrm{Sc}, \mathrm{Y}) . R S C$ Adv. 5, 1366-1377 (2015).

[85] Taddee, C., Kamwanna, T. \& Amornkitbamrung, V. Characterization of transparent superconductivity Fe-doped $\mathrm{CuCrO}_{2}$ delafossite oxide. Applied Surface Science 380, 237-242 (2016).

[86] Zazoua, H. et al. Selective gas-phase hydrogenation of benzaldehyde in the presence of Ni-, Co- and Fe-doped $\mathrm{BaCuO}_{2}$ delafossites: effect of metal substituent on the production of benzyl alcohol. Research on Chemical Intermediates (2019). 
[87] Kurita, K., Sakabayashi, H. \& Okazaki, R. Correlation in transport coefficients of hole-doped $\mathrm{CuRhO}_{2}$ single crystals. Phys. Rev. B 99, 115103 (2019).

[88] Hwang, B.-Y., Sakthinathan, S. \& Chiu, T.-W. Production of hydrogen from steam reforming of methanol carried out by self-combusted $\mathrm{CuCr}_{1-x} \mathrm{Fe}_{x} \mathrm{O}_{2} \quad$ (x $=0-1$ ) nanopowders catalyst. International Journal of Hydrogen Energy 44, $2848-2856$ (2019).

[89] Dong, H. et al. Visible light-induced photocatalytic activity of delafossite $\mathrm{AgMO}_{2}(\mathrm{M}=\mathrm{Al}, \mathrm{Ga}, \mathrm{In})$ prepared via a hydrothermal method. Applied Catalysis B: Environmental 89, 551-556 (2009).

[90] Lekse, J. W., Underwood, M. K., Lewis, J. P. \& Matranga, C. Synthesis, characterization, electronic structure, and photocatalytic behavior of $\mathrm{CuGaO}_{2}$ and $\mathrm{CuGa}_{1-x} \mathrm{Fe}_{x} \mathrm{O}_{2}(\mathrm{x}=0.05,0.10,0.15,0.20)$ delafossites. The Journal of Physical Chemistry C 116, 1865-1872 (2012).

[91] Lekse, J. W., Haycock, B. J., Lewis, J. P., Kauffman, D. R. \& Matranga, C. The effect of electronic structure changes in $\mathrm{NaInO}_{2}$ and $\mathrm{NaIn}_{0.9} \mathrm{Fe}_{0.1} \mathrm{O}_{2}$ on the photoreduction of methylene blue. J. Mater. Chem. A 2, 9331-9337 (2014).

[92] Yin, L. et al. A novel delafossite structured visible-light sensitive $\mathrm{AgFeO}_{2}$ photocatalyst: Preparation, photocatalytic properties, and reaction mechanism. Catalysts 6 (2016).

[93] Shi, L. et al. Photoluminescence and photocatalytic properties of rhombohedral $\mathrm{CuGaO}_{2}$ nanoplates. Scientific Reports 6, 21135 EP - (2016). Article.

[94] Ahmad, T. et al. Dielectric, optical and enhanced photocatalytic properties of $\mathrm{CuCrO}_{2}$ nanoparticles. RSC Adv. 7, 27549-27557 (2017).

[95] Huda, M. N., Yan, Y., Walsh, A., Wei, S.-H. \& Al-Jassim, M. M. Symmetrybreaking-induced enhancement of visible light absorption in delafossite alloys. Applied Physics Letters 94, 251907 (2009).

[96] Maurice, D. \& Head-Gordon, M. Analytical second derivatives for excited electronic states using the single excitation configuration interaction method: theory and application to benzo[a]pyrene and chalcone. Molecular Physics 96, 1533-1541 (1999).

[97] Head-Gordon, M., Rico, R. J., Oumi, M. \& Lee, T. J. A doubles correction to electronic excited states from configuration interaction in the space of single substitutions. Chemical Physics Letters 219, 21 - 29 (1994). 
[98] Purvis, G. D. \& Bartlett, R. J. A full coupled-cluster singles and double model: The inclusion of disconnected triples. The journal of Chemical Physics 76, 1910-1918 (1982).

[99] Raghavachari, K., Trucks, G. W., Pople, J. A. \& Head-Gordon, M. A fifth-order perturbation comparison of electron correlation theories. Chemical Physics Letters 157, $479-483$ (1989).

[100] Meyer, H.-D., Manthe, U. \& Cederbaum, L. The multi-configurational timedependent hartree approach. Chemical Physics Letters 165, 73 - 78 (1990).

[101] Pople, J. A., Head-Gordon, M. \& Raghavachari, K. Quadratic configuration interaction. a general technique for determining electron correlation energies. The journal of Chemical Physics 87, 5968-5975 (1987).

[102] Kohn, W. \& Sham, L. J. Self-consistent equations including exchange and correlation effects. Phys. Rev. 140, A1133-A1138 (1965).

[103] Parr, R. G. \& Weitao, Y. Density-Functional Theory of Atoms and Molecules (Oxford University Press, 1994).

[104] Sankey, O. F. \& Niklewski, D. J. Ab initio multicenter tight-binding model for molecular-dynamics simulations and other applications in covalent systems. Physical Review B 40, 3979-3995 (1989).

[105] Finnis, M. W. The harris functional applied to surface and vacancy formation energies in aluminium. Journal of Physics: Condensed Matter 2, 331 (1990).

[106] Lewis, J. P. et al. Advances and applications in the FIREBALL ab initio tightbinding molecular-dynamics formalism. physica status solidi (b) 248, 1989-2007 (2011).

[107] Demkov, A. A., Ortega, J., Sankey, O. F. \& Grumbach, M. P. Electronic structure approach for complex silicas. Phys. Rev. B 52, 1618-1630 (1995).

[108] Becke, A. D. Density-functional exchange-energy approximation with correct asymptotic behavior. Phys. Rev. A 38, 3098-3100 (1988).

[109] Lee, C., Yang, W. \& Parr, R. G. Development of the colle-salvetti correlationenergy formula into a functional of the electron density. Phys. Rev. B 37, 785-789 (1988). 
[110] Langreth, D. C. \& Mehl, M. J. Beyond the local-density approximation in calculations of ground-state electronic properties. Phys. Rev. B 28, 1809-1834 (1983).

[111] Perdew, J. P. et al. Atoms, molecules, solids, and surfaces: Applications of the generalized gradient approximation for exchange and correlation. Phys. Rev. B 46, 6671-6687 (1992).

[112] Hamann, D. R., Schlüter, M. \& Chiang, C. Norm-conserving pseudopotentials. Phys. Rev. Lett. 43, 1494-1497 (1979).

[113] Troullier, N. \& Martins, J. L. Efficient pseudopotentials for plane-wave calculations. Phys. Rev. B 43, 1993-2006 (1991).

[114] Heaven, M. W., Dass, A., White, P. S., Holt, K. M. \& Murray, R. W. Crystal structure of the gold nanoparticle $\left[\mathrm{N}\left(\mathrm{C}_{8} \mathrm{H}_{17}\right)_{4}\right]\left[\mathrm{Au}_{25}\left(\mathrm{SCH}_{2} \mathrm{CH}_{2} \mathrm{Ph}\right)_{18}\right]$. Journal of the American Chemical Society 130, 3754-3755 (2008).

[115] Guidez, E. B., Mäkinen, V., Häkkinen, H. \& Aikens, C. M. Effects of silver doping on the geometric and electronic structure and optical absorption spectra of the $\mathrm{Au}_{25-n} \mathrm{Ag}_{n}(\mathrm{SH})_{18}{ }^{-}(\mathrm{n}=1,2,4,6,8,10,12)$ bimetallic nanoclusters. The Journal of Physical Chemistry C 116, 20617-20624 (2012).

[116] Tsukuda, T. Toward an atomic-level understanding of size-specific properties of protected and stabilized gold clusters. Bulletin of the Chemical Society of Japan 85, 151-168 (2012).

[117] Negishi, Y. Toward the creation of functionalized metal nanoclusters and highly active photocatalytic materials using thiolate-protected magic gold clusters. Bulletin of the Chemical Society of Japan 87, 375-389 (2014).

[118] Xu, W. W., Zhu, B., Zeng, X. C. \& Gao, Y. A grand unified model for liganded gold clusters. Nature Communications 7, 13574 (2016).

[119] Jin, R. et al. Controlling anisotropic nanoparticle growth through plasmon excitation. Nature 425, 487 (2003).

[120] Rosi, N. L. \& Mirkin, C. A. Nanostructures in biodiagnostics. Chemical reviews 105, 1547-62 (2005).

[121] Liu, Y., Tsunoyama, H., Akita, T. \& Tsukuda, T. Efficient and selective epoxidation of styrene with TBHP catalyzed by $\mathrm{Au}_{25}$ clusters on hydroxyapatite. Chemical Communications 46, 550-552 (2010). 
[122] Li, G. \& Jin, R. Atomically precise gold nanoclusters as new model catalysts. Accounts of Chemical Research 46, 1749-1758 (2013).

[123] Jin, R. \& Nobusada, K. Doping and alloying in atomically precise gold nanoparticles. Nano Research 7, 285-300 (2014).

[124] Parker, J. F., Fields-Zinna, C. A. \& Murray, R. W. The story of a monodisperse gold nanoparticle: $\mathrm{Au}_{25} \mathrm{~L}_{18}$. Accounts of chemical research 43, 1289-96 (2010).

[125] MacDonald, M. A., Chevrier, D. M., Zhang, P., Qian, H. \& Jin, R. The structure and bonding of $\mathrm{Au}_{25}(\mathrm{SR})_{18}$ nanoclusters from EXAFS: The interplay of metallic and molecular behavior. The Journal of Physical Chemistry $C \mathbf{1 1 5}$, 15282-15287 (2011).

[126] Antonello, S., Perera, N. V., Ruzzi, M., Gascón, J. A. \& Maran, F. Interplay of charge state, lability, and magnetism in the molecule-like $\mathrm{Au}_{25}(\mathrm{SR})_{18}$ cluster. Journal of the American Chemical Society 135, 15585-15594 (2013).

[127] De Nardi, M. et al. Gold nanowired: A linear $\left(\mathrm{Au}_{25}\right)_{n}$ polymer from $\mathrm{Au}_{25}$ molecular clusters. ACS Nano 8, 8505-8512 (2014).

[128] Akola, J., Walter, M., Whetten, R. L., Häkkinen, H. \& Grönbeck, H. On the structure of thiolate-protected $\mathrm{Au}_{25}$. Journal of the American Chemical Society 130, 3756-3757 (2008).

[129] Pei, Y. \& Zeng, X. C. Investigating the structural evolution of thiolate protected gold clusters from first-principles. Nanoscale 4, 4054-4072 (2012).

[130] Christensen, S. L. et al. Dopant location, local structure, and electronic properties of $\mathrm{Au}_{24} \mathrm{Pt}(\mathrm{SR})_{18}$ nanoclusters. The Journal of Physical Chemistry $C \mathbf{1 1 6}$, 26932-26937 (2012).

[131] Yamazoe, S., Kurashige, W., Nobusada, K., Negishi, Y. \& Tsukuda, T. Preferential location of coinage metal dopants $(\mathrm{M}=\mathrm{Ag}$ or $\mathrm{Cu})$ in $\left[\mathrm{Au}_{25-x} \mathrm{M}_{x}\left(\mathrm{SC}_{2} \mathrm{H}_{4} \mathrm{Ph}\right)_{18}\right]^{-}$ $(\mathrm{x} \sim 1)$ as determined by extended x-ray absorption fine structure and density functional theory calculations. The Journal of Physical Chemistry $C \mathbf{1 1 8}$, 25284-25290 (2014).

[132] Tian, S. et al. Structures and magnetism of mono-palladium and mono-platinum doped $\mathrm{Au}_{25}(\mathrm{PET})_{18}$ nanoclusters. Chem. Commun. 52, 9873-9876 (2016).

[133] Jiang, D.-e. \& Dai, S. From superatomic $\mathrm{Au}_{25}(\mathrm{SR})_{18}{ }^{-}$to $\mathrm{M} @ \mathrm{Au}_{24}(\mathrm{SR})_{18}{ }^{q}$ CoreShell clusters. Inorganic Chemistry 48, 2720-2722 (2009). 
[134] Miller, S. A., Fields-Zinna, C. A., Murray, R. W. \& Moran, A. M. Nonlinear optical signatures of core and ligand electronic states in au24pdl18 (2010).

[135] Walter, M. \& Moseler, M. Ligand-protected gold alloy clusters: Doping the superatom. The Journal of Physical Chemistry C 113, 15834-15837 (2009).

[136] Tlahuice-Flores, A. Optical properties of thiolate-protected $\mathrm{Ag}_{n} \mathrm{Au}_{25-n}\left(\mathrm{SCH}_{3}\right)_{18}^{-}$ clusters. Journal of Nanoparticle Research 15, 1771 (2013).

[137] Kauffman, D. R., Alfonso, D., Matranga, C., Qian, H. \& Jin, R. A quantum alloy: The ligand-protected $\operatorname{Au}_{2} 5_{x} \operatorname{Ag}_{x}(\mathrm{SR})_{18}$ cluster. The Journal of Physical Chemistry C 117, 7914-7923 (2013).

[138] Li, G., Jiang, D.-e., Liu, C., Yu, C. \& Jin, R. Oxide-supported atomically precise gold nanocluster for catalyzing sonogashira cross-coupling. Journal of Catalysis 306, 177-183 (2013).

[139] Li, G., Jiang, D.-e., Kumar, S., Chen, Y. \& Jin, R. Size dependence of atomically precise gold nanoclusters in chemoselective hydrogenation and active site structure. ACS Catalysis 4, 2463-2469 (2014).

[140] Ouyang, R. \& Jiang, D.-e. Understanding selective hydrogenation of $\alpha, \beta-$ unsaturated ketones to unsaturated alcohols on the $\mathrm{Au}_{25}(\mathrm{SR})_{18}$ cluster. ACS Catalysis 5, 6624-6629 (2015).

[141] Li, G., Abroshan, H., Chen, Y., Jin, R. \& Kim, H. J. Experimental and mechanistic understanding of aldehyde hydrogenation using $\mathrm{Au}_{25}$ nanoclusters with lewis acids: Unique sites for catalytic reactions. Journal of the American Chemical Society 137, 14295-14304 (2015).

[142] Li, G. \& Jin, R. Gold nanocluster-catalyzed semihydrogenation: A unique activation pathway for terminal alkynes. Journal of the American Chemical Society 136, 11347-11354 (2014).

[143] Li, G. \& Jin, R. Atomic level tuning of the catalytic properties: Doping effects of 25-atom bimetallic nanoclusters on styrene oxidation. Catalysis Today 278, 187-191 (2016).

[144] Li, Z., Yang, X., Liu, C., Wang, J. \& Li, G. Effects of doping in 25-atom bimetallic nanocluster catalysts for carbon-carbon coupling reaction of iodoanisole and phenylacetylene. Progress in Natural Science: Materials International 26, 477482 (2016). 
[145] Li, W. et al. Catalytic CO oxidation using bimetallic $\mathrm{M}_{\mathrm{x}} \mathrm{Au}_{25-\mathrm{x}}$ clusters: A combined experimental and computational study on doping effects. The Journal of Physical Chemistry C 120, 10261-10267 (2016-05).

[146] Keith, J. B., Fennick, J. R., Junkermeier, C. E., Nelson, D. R. \& Lewis, J. P. A web-deployed interface for performing ab initio molecular dynamics, optimization, and electronic structure in fireball. Computer Physics Communications 180, 418-426 (2009).

[147] Jelínek, P., Wang, H., Lewis, J. P., Sankey, O. F. \& Ortega, J. Multicenter approach to the exchange-correlation interactions in ab initio tight-binding methods. Physical Review B 71, 235101 (2005).

[148] Grönbeck, H., Curioni, A. \& Andreoni, W. Thiols and disulfides on the Au(111) surface: the headgroup-gold interaction. Journal of the American Chemical Society 122, 3839-3842 (2000).

[149] Fukui, K., Yonezawa, T. \& Shingu, H. A molecular orbital theory of reactivity in aromatic hydrocarbons. The Journal of Chemical Physics 20, 722-725 (1952).

[150] Fukui, K., Yonezawa, T., Nagata, C. \& Shingu, H. Molecular orbital theory of orientation in aromatic, heteroaromatic, and other conjugated molecules. The Journal of Chemical Physics 22, 1433-1442 (1954).

[151] Mulliken, R. S. Electronic population analysis on lcao-mo molecular wave functions. i. The Journal of Chemical Physics 23, 1833-1840 (1955).

[152] Reed, A. E., Curtiss, L. A. \& Weinhold, F. Intermolecular interactions from a natural bond orbital, donor-acceptor viewpoint. Chemical Reviews 88, 899-926 (1988).

[153] Hirshfeld, F. L. Bonded-atom fragments for describing molecular charge densities. Theoretica chimica acta 44, 129-138 (1977).

[154] Tang, W., Sanville, E. \& Henkelman, G. A grid-based bader analysis algorithm without lattice bias. Journal of Physics: Condensed Matter 21, 084204 (2009).

[155] Löwdin, P.-O. On the non-orthogonality problem connected with the use of atomic wave functions in the theory of molecules and crystals. The Journal of Chemical Physics 18, 365-375 (1950). 
[156] Senet, P. Kohn-sham orbital formulation of the chemical electronic responses, including the hardness. The Journal of Chemical Physics 107, 2516-2524 (1997).

[157] Contreras, R. R., Fuentealba, P., Galván, M. \& Pérez, P. A direct evaluation of regional fukui functions in molecules. Chemical Physics Letters 304, 405 413 (1999).

[158] Chandra, A. K. \& Nguyen, M. T. Chemical Reactivity Theory: A Density Functional View (CRC Press, 2009).

[159] de Heer, W. A. The physics of simple metal clusters: experimental aspects and simple models. Rev. Mod. Phys. 65, 611-676 (1993).

[160] Walter, M. et al. A unified view of ligand-protected gold clusters as superatom complexes. Proceedings of the National Academy of Sciences 105, 9157-9162 (2008).

[161] Allison, T. C. \& Tong, Y. J. Application of the condensed fukui function to predict reactivity in core-shell transition metal nanoparticles. Electrochimica Acta 101, 334 - 340 (2013).

[162] Nie, X., Qian, H., Ge, Q., Xu, H. \& Jin, R. CO oxidation catalyzed by oxidesupported $\mathrm{Au}_{25}(\mathrm{SR})_{18}$ nanoclusters and identification of perimeter sites as active centers. ACS Nano 6, 6014-6022 (2012).

[163] Zhu, M., Eckenhoff, W. T., Pintauer, T. \& Jin, R. Conversion of anionic $\left[\mathrm{Au}_{25}\left(\mathrm{SCH}_{2} \mathrm{CH}_{2} \mathrm{Ph}\right)_{18}\right]^{-}$cluster to charge neutral cluster via air oxidation. The Journal of Physical Chemistry C 112, 14221-14224 (2008-09).

[164] Jin, R. et al. Controlling Ag-doping in $\left[\mathrm{Ag}_{x} \mathrm{Au}_{25-x}\left(\mathrm{SC}_{6} \mathrm{H}_{11}\right)^{18}\right]^{-}$nanoclusters: cryogenic optical, electronic and electrocatalytic properties. Nanoscale $\mathbf{9}$, 19183-19190 (2017).

[165] Taylor, M. G. \& Mpourmpakis, G. Thermodynamic stability of ligand-protected metal nanoclusters. Nature Communications 8, 15988 (2017).

[166] Shuang, C. et al. The structure and optical properties of the $\left[\mathrm{Au}_{18}(\mathrm{SR})_{14}\right]$ nanocluster. Angewandte Chemie International Edition 54, 3145-3149 (2015).

[167] Pei, Y., Gao, Y., Shao, N. \& Zeng, X. C. Thiolate-protected $\mathrm{Au}_{20}(\mathrm{SR})_{16}$ cluster: Prolate $\mathrm{au}_{8}$ core with new $\left[\mathrm{Au}_{3}(\mathrm{SR})_{4}\right]$ staple motif. Journal of the American Chemical Society 131, 13619-13621 (2009). 
[168] Jiang, D.-e., Chen, W., Whetten, R. L. \& Chen, Z. What protects the core when the thiolated Au cluster is extremely small? The Journal of Physical Chemistry C 113, 16983-16987 (2009).

[169] Zeng, C., Liu, C., Chen, Y., Rosi, N. L. \& Jin, R. Gold-thiolate ring as a protecting motif in the $\mathrm{Au}_{20}(\mathrm{SR})_{16}$ nanocluster and implications. Journal of the American Chemical Society 136, 11922-11925 (2014). PMID: 25126666.

[170] Zhu, M., Qian, H. \& Jin, R. Thiolate-protected $\mathrm{Au}_{24}\left(\mathrm{SC}_{2} \mathrm{H}_{4} \mathrm{Ph}\right)_{20}$ nanoclusters: Superatoms or not? The Journal of Physical Chemistry Letters 1, 1003-1007 (2010).

[171] Dass, A. et al. Crystal structure and theoretical analysis of green gold $\mathrm{Au}_{30}(\mathrm{~S}-$ $\mathrm{tBu})_{18}$ nanomolecules and their relation to $\mathrm{Au}_{30} \mathrm{~S}(\mathrm{~S}-\mathrm{tBu})_{18}$. The Journal of Physical Chemistry C 120, 6256-6261 (2016).

[172] Tatsuya, H. et al. Controlling the atomic structure of $\mathrm{Au}_{30}$ nanoclusters by a ligand based strategy. Angewandte Chemie International Edition 55, 6694-6697 (2016).

[173] Chenjie, Z. et al. Total structure and electronic properties of the gold nanocrystal $\mathrm{Au}_{36}(\mathrm{SR})_{24}$. Angewandte Chemie International Edition 51, 13114-13118 (2012).

[174] Chevrier, D. M., Chatt, A., Zhang, P., Zeng, C. \& Jin, R. Unique bonding properties of the $\mathrm{Au}_{36}(\mathrm{SR})_{24}$ nanocluster with fcc-like core. The Journal of Physical Chemistry Letters 4, 3186-3191 (2013).

[175] Qian, H., Eckenhoff, W. T., Zhu, Y., Pintauer, T. \& Jin, R. Total structure determination of thiolate-protected $\mathrm{Au}_{38}$ nanoparticles. Journal of the American Chemical Society 132, 8280-1 (2010).

[176] Pei, Y., Gao, Y. \& Zeng, X. C. Structural prediction of thiolate-protected Au un $_{38}$ : A face-fused bi-icosahedral au core. Journal of the American Chemical Society 130, 7830-7832 (2008).

[177] Lopez-Acevedo, O., Tsunoyama, H., Tsukuda, T., Häkkinen, H. \& Aikens, C. M. Chirality and electronic structure of the thiolate-protected $\mathrm{Au}_{38}$ nanocluster. Journal of the American Chemical Society 132, 8210-8218 (2010).

[178] Jung, J., Kang, S. \& Han, Y.-K. Ligand effects on the stability of thiol-stabilized gold nanoclusters: Au25(sr)18-, au38(sr)24, and au102(sr)44. Nanoscale 4, 4206-4210 (2012). 
[179] Gao, Y., Shao, N. \& Zeng, X. C. Ab initio study of thiolate-protected Au $\mathrm{u}_{102}$ nanocluster. ACS Nano 2, 1497-1503 (2008).

[180] Li, Y., Galli, G. \& Gygi, F. Electronic structure of thiolate-covered gold nanoparticles: $\mathrm{Au}_{102}(\mathrm{MBA})_{44}$. ACS Nano 2, 1896-1902 (2008).

[181] Hulkko, E. et al. Electronic and vibrational signatures of the $\mathrm{Au}_{102}(\mathrm{p}-\mathrm{MBA})_{44}$ cluster. Journal of the American Chemical Society 133, 3752-3755 (2011).

[182] Shichibu, Y. et al. Extremely high stability of glutathionate-protected $\mathrm{Au}_{25}$ clusters against core etching. Small 3, 835-839 (2007).

[183] Ghosh, A., Udayabhaskararao, T. \& Pradeep, T. One-step route to luminescent $\mathrm{Au}_{18} \mathrm{SG}_{14}$ in the condensed phase and its closed shell molecular ions in the gas phase. The Journal of Physical Chemistry Letters 3, 1997-2002 (2012).

[184] Das, A. et al. Crystal structure and electronic properties of a thiolate-protected $\mathrm{Au}_{24}$ nanocluster. Nanoscale 6, 6458-6462 (2014).

[185] $\mathrm{Yu}, \mathrm{Y}$. et al. Identification of a highly luminescent $\mathrm{Au}_{22}(\mathrm{SG})_{18}$ nanocluster. Journal of the American Chemical Society 136, 1246-1249 (2014).

[186] Zhu, M. et al. Reversible switching of magnetism in thiolate-protected $\mathrm{Au}_{25}$ superatoms. Journal of the American Chemical Society 131, 2490-2492 (2009).

[187] McCoy, R. S., Choi, S., Collins, G., Ackerson, B. J. \& Ackerson, C. J. Superatom paramagnetism enables gold nanocluster heating in applied radiofrequency fields. ACS Nano 7, 2610-2616 (2013).

[188] Ramakrishna, G., Varnavski, O., Kim, J., Lee, D. \& Goodson, T. Quantumsized gold clusters as efficient two-photon absorbers. Journal of the American Chemical Society 130, 5032-5033 (2008).

[189] Russier-Antoine, I. et al. Non-linear optical properties of gold quantum clusters. the smaller the better. Nanoscale 6, 13572-13578 (2014).

[190] Knoppe, S. et al. Nonlinear optical properties of thiolate-protected gold clusters. The Journal of Physical Chemistry C 119, 6221-6226 (2015).

[191] Day, P. N., Pachter, R., Nguyen, K. A. \& Bigioni, T. P. Linear and nonlinear optical response in silver nanoclusters: Insight from a computational investigation. The Journal of Physical Chemistry A 120, 507-518 (2016). 
[192] Jin, R., Zeng, C., Zhou, M. \& Chen, Y. Atomically precise colloidal metal nanoclusters and nanoparticles: Fundamentals and opportunities. Chemical Reviews 116, 10346-10413 (2016).

[193] Mpourmpakis, G., Andriotis, A. N. \& Vlachos, D. G. Identification of descriptors for the $\mathrm{CO}$ interaction with metal nanoparticles. Nano Letters $\mathbf{1 0}$, 1041-1045 (2010).

[194] Federico, C., I., M. J., M., G. J., Philippe, S. \& David, L. Fast prediction of adsorption properties for platinum nanocatalysts with generalized coordination numbers. Angewandte Chemie International Edition 53, 8316-8319 (2014).

[195] Calle-Vallejo, F., Loffreda, D., Koper, M. T. M. \& Sautet, P. Introducing structural sensitivity into adsorption-energy scaling relations by means of coordination numbers. Nature Chemistry 7, 403 (2015).

[196] Calle-Vallejo, F. et al. Finding optimal surface sites on heterogeneous catalysts by counting nearest neighbors. Science 350, 185-189 (2015).

[197] Ma, X. \& Xin, H. Orbitalwise coordination number for predicting adsorption properties of metal nanocatalysts. Phys. Rev. Lett. 118, 036101 (2017).

[198] Wu, Z. et al. Thiolate ligands as a double-edged sword for CO oxidation on $\mathrm{CeO}_{2}$ supported $\mathrm{Au}_{25}\left(\mathrm{SCH}_{2} \mathrm{CH}_{2} \mathrm{Ph}\right)_{18}$ nanoclusters. Journal of the American Chemical Society 136, 6111-6122 (2014).

[199] Haruta, M., Kobayashi, T., Sano, H. \& Yamada, N. Novel gold catalysts for the oxidation of carbon monoxide at a temperature far below $0^{\circ} \mathrm{C}$. Chemistry Letters 16, 405-408 (1987).

[200] Green, I. X., Tang, W., Neurock, M. \& Yates, J. T. Spectroscopic observation of dual catalytic sites during oxidation of $\mathrm{CO}$ on a $\mathrm{Au} / \mathrm{TiO}_{2}$ catalyst. Science 333, 736-739 (2011).

[201] Gao, F., Wood, T. E. \& Goodman, D. W. The effects of water on CO oxidation over $\mathrm{TiO}_{2}$ supported au catalysts. Catalysis Letters 134, 9-12 (2010).

[202] Panapitiya, G. et al. Structural and catalytic properties of the $\mathrm{Au}_{25-x} \mathrm{Ag}_{x}\left(\mathrm{SCH}_{3}\right)_{18}$ $(\mathrm{x}=6,7,8)$ nanocluster. Phys. Chem. Chem. Phys. 20, 13747-13756 (2018).

[203] Mitchell, T. M. Machine Learning (McGraw-Hill Education, 1997). 
[204] Pereira, F., Latino, D. A. R. S. \& Aires-de Sousa, J. Estimation of mayr electrophilicity with a quantitative structure-property relationship approach using empirical and DFT descriptors. The Journal of Organic Chemistry 76, 9312-9319 (2011).

[205] Rupp, M., Tkatchenko, A., Müller, K.-R. \& von Lilienfeld, O. A. Fast and accurate modeling of molecular atomization energies with machine learning. Physical Review Letters 108, 058301 (2012).

[206] Huan, T. D., Mannodi-Kanakkithodi, A. \& Ramprasad, R. Accelerated materials property predictions and design using motif-based fingerprints. Physical Review B 92, 014106 (2015).

[207] Zhang, Q., Zheng, F., Zhao, T., Qu, X. \& Aires-de Sousa, J. Machine learning estimation of atom condensed fukui functions. Molecular Informatics 35, 62-69 (2016).

[208] Mannodi-Kanakkithodi, A., Pilania, G., Huan, T. D., Lookman, T. \& Ramprasad, R. Machine learning strategy for accelerated design of polymer dielectrics 6, 20952 (2016).

[209] Pilania, G. et al. Machine learning bandgaps of double perovskites. Scientific Reports 6, 19375 (2016).

[210] Borboudakis, G. et al. Chemically intuited, large-scale screening of MOFs by machine learning techniques. npj Computational Materials 3, 40 (2017).

[211] Pereira, F. et al. Machine learning methods to predict density functional theory B3LYP energies of HOMO and LUMO orbitals. Journal of Chemical Information and Modeling 57, 11-21 (2017).

[212] Xu, W. W., Gao, Y. \& Zeng, X. C. Unraveling structures of protection ligands on gold nanoparticle $\mathrm{Au}_{68}(\mathrm{SH})_{32}$. Science Advances 1 (2015).

[213] Karki, I. et al. Tripeptides on gold nanoparticles: Structural differences between two reverse sequences as determined by solid-state $\mathrm{nmr}$ and dft calculations. The Journal of Physical Chemistry B 119, 11998-12006 (2015).

[214] Ranasingha, O. et al. Slow relaxation of surface plasmon excitations in $\mathrm{Au}_{55}$ : The key to efficient plasmonic heating in $\mathrm{Au} / \mathrm{TiO}_{2}$. Journal of Physical Chemistry Letters 7 (2016). 
[215] Carr, J. A., Wang, H., Abraham, A., Gullion, T. \& Lewis, J. P. l-cysteine interaction with au55 nanoparticle. The Journal of Physical Chemistry C 116, 25816-25823 (2012).

[216] Wadell, H. Volume, shape, and roundness of quartz particles. The Journal of Geology 43, 250-280 (1935).

[217] Banerjee, A. N. \& Chattopadhyay, K. K. Size-dependent optical properties of sputter-deposited nanocrystalline p-type transparent $\mathrm{CuAlO} 2$ thin films. Journal of Applied Physics 97, 084308 (2005).

[218] Rastogi, A. C., Lim, S. H. \& Desu, S. B. Structure and optoelectronic properties of spray deposited mg doped p-CuCrO2 semiconductor oxide thin films. Journal of Applied Physics 104, 023712 (2008).

[219] Hiraga, H., Makino, T., Fukumura, T., Ohtomo, A. \& Kawasaki, M. Excitonic characteristics in direct wide-band-gap $\mathrm{CuScO}_{2}$ epitaxial thin films. Applied Physics Letters 95, 211908 (2009).

[220] Younsi, M., Saadi, S., Bouguelia, A., Aider, A. \& Trari, M. Synthesis and characterization of oxygen-rich delafossite $\mathrm{CuYO}_{2+x}$-Application to $\mathrm{H}_{2}$-photo production. Solar Energy Materials and Solar Cells 91, 1102-1109 (2007).

[221] Saadi, S., Bouguelia, A. \& Trari, M. Photocatalytic hydrogen evolution over $\mathrm{CuCrO}_{2}$. Solar Energy 80, 272-280 (2006).

[222] Saadi, S., Bouguelia, A., Derbal, A. \& Trari, M. Hydrogen photoproduction over new catalyst $\mathrm{CuLaO}_{2}$. Journal of Photochemistry and Photobiology A: Chemistry 187, 97-104 (2007).

[223] Farley, K. E., Marschilok, A. C., Takeuchi, E. S. \& Takeuchi, K. J. Synthesis and electrochemistry of silver ferrite. Electrochemical and Solid-State Letters 15, A23-A27 (2011).

[224] Durham, J. L., Kirshenbaum, K., Takeuchi, E. S., Marschilok, A. C. \& Takeuchi, K. J. Synthetic control of composition and crystallite size of silver ferrite composites: profound electrochemistry impacts. Chemical Communications 51, 5120-5123 (2015).

[225] Yagi, H., Seo, W. S. \& Koumoto, K. Thermoelectric properties of delafossitetype oxides 181, 63-68 (2000). 
[226] Koumoto, K., Koduka, H. \& Seo, W.-S. Thermoelectric properties of single crystal $\mathrm{CuAlO}_{2}$ with a layered structure. Journal of Materials Chemistry 11, 251-252 (2001).

[227] Doumerc, J. P. et al. Transition-metal oxides for thermoelectric generation. Journal of Electronic Materials 38, 1078-1082 (2009).

[228] Lalanne, M., Barnabé, A., Mathieu, F. \& Tailhades, P. Synthesis and thermostructural studies of a $\mathrm{CuFe}_{1-\mathrm{x}} \mathrm{Cr}_{\mathrm{x}} \mathrm{O}_{2}$ delafossite solid solution with $0 \leq \mathrm{x} \leq$ 1. Inorganic Chemistry 48, 6065-6071 (2009).

[229] Nie, X., Wei, S.-H. \& Zhang, S. B. Bipolar doping and band-gap anomalies in delafossite transparent conductive oxides. Phys. Rev. Lett. 88, 066405 (2002).

[230] Senty, T. R. et al. Optical absorption and disorder in delafossites. Applied Physics Letters 111 (2017).

[231] Beznosikov, B. V. \& Aleksandrov, K. S. Predictions of compounds in the family of delafossites. Journal of Structural Chemistry 50, 102-107 (2009).

[232] Maruyama, Y., Irie, H. \& Hashimoto, K. Visible light sensitive photocatalyst, delafossite structured $\alpha$-aggao2. The Journal of Physical Chemistry B 110, 23274-23278 (2006).

[233] Kumar, M. \& Persson, C. Structural, electronic and optical properties of silver delafossite oxides: A first-principles study with hybrid functional. Physica B: Condensed Matter 422, 20 - 27 (2013).

[234] Denton, A. R. \& Ashcroft, N. W. Vegard's law. Phys. Rev. A 43, 3161-3164 (1991).

[235] Nie, X., Wei, S.-H. \& Zhang, S. B. Bipolar doping and band-gap anomalies in delafossite transparent conductive oxides. Physical Review Letters 88, 066405 (2002).

[236] Giannozzi, P. et al. QUANTUM ESPRESSO: a modular and open-source software project for quantum simulations of materials. Journal of Physics: Condensed Matter 21, 395502 (2009).

[237] Morgan, B. J. bsym: A basic symmetry module. The Journal of Open Source Software 2 (2017). URL https://doi.org/10.21105/joss.00370. 
[238] Medeiros, P. V. C., Stafström, S. \& Björk, J. Effects of extrinsic and intrinsic perturbations on the electronic structure of graphene: Retaining an effective primitive cell band structure by band unfolding. Phys. Rev. B 89, 041407 (2014).

[239] Medeiros, P. V. C., Tsirkin, S. S., Stafström, S. \& Björk, J. Unfolding spinor wave functions and expectation values of general operators: Introducing the unfolding-density operator. Phys. Rev. B 91, 041116 (2015).

[240] Laporte, O. \& Meggers, W. F. Some rules of spectral structure. J. Opt. Soc. Am. 11, 459-463 (1925).

[241] Henderson, B. \& Imbusch, G. F. Optical Spectroscopy of Inorganic Solids (Monographs on the Physics and Chemistry of Materials) (Oxford University Press, 2006).

[242] Pedregosa, F. et al. Scikit-learn: Machine learning in python. Journal of Machine Learning Research 12, 2825-2830 (2011). 
APPENDICES 


\section{APPENDIX A}

\section{Low energy isomers of 6-8 $\mathrm{Ag}_{x} \mathrm{Au}_{25-x}\left(\mathrm{SCH}_{3}\right)_{18}$}
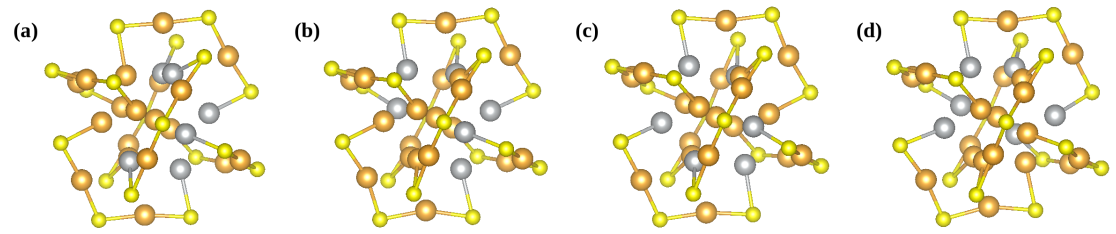

(a) Lowest energy 6-Ag alloyed $\mathrm{Au}_{25}\left(\mathrm{SCH}_{3}\right)_{18}$
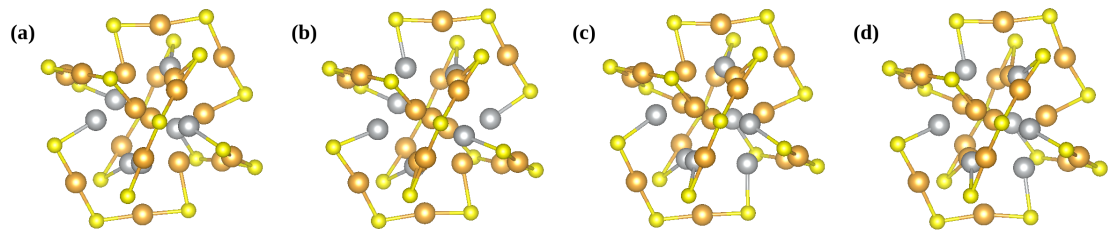

(b) Lowest energy 7-Ag alloyed $\mathrm{Au}_{25}\left(\mathrm{SCH}_{3}\right)_{18}$
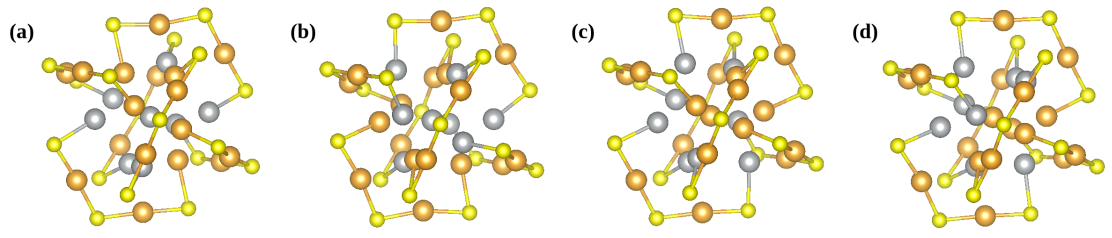

(c) Lowest energy 8-Ag alloyed $\mathrm{Au}_{25}\left(\mathrm{SCH}_{3}\right)_{18}$

Figure A.1: Figures (a)-(c) depicts the Ag positions in $2^{\text {nd }}$ to $5^{\text {th }}$ lowest energy isomers for $x=6-8$. Symmetries in the unalloyed $\mathrm{Au}_{25}$ are found to be broken due to $\mathrm{Ag}$ atoms. 
Bar plots showing the Ag occupations in the three structural units in $\operatorname{Ag}_{x} \mathrm{Au}_{25-x}(\mathrm{SR})_{18}$ for $x=3-22$.

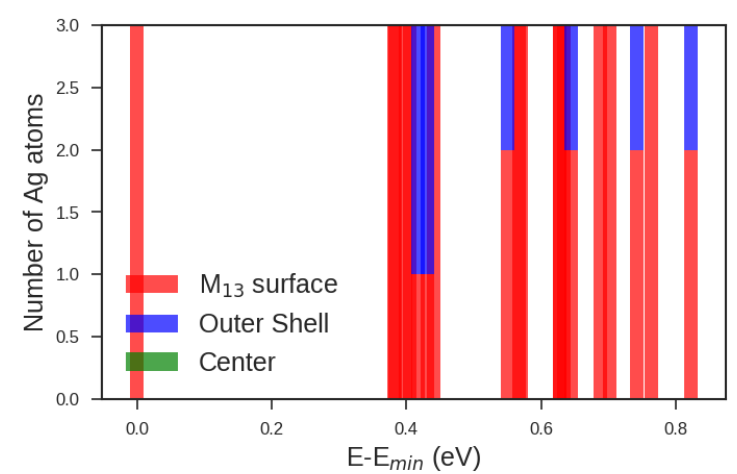

(a)

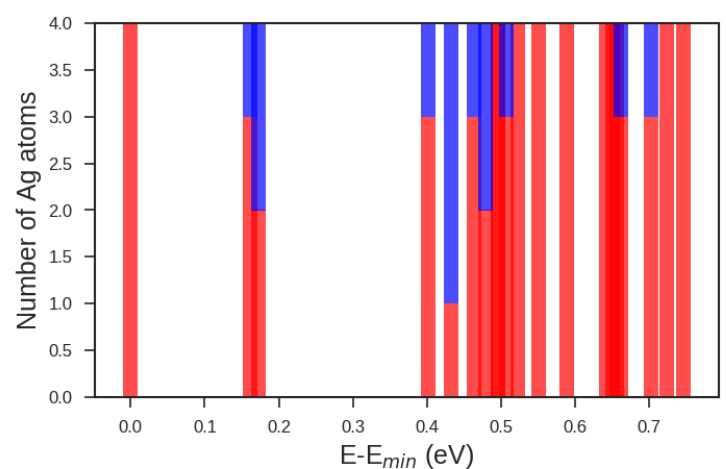

(b)

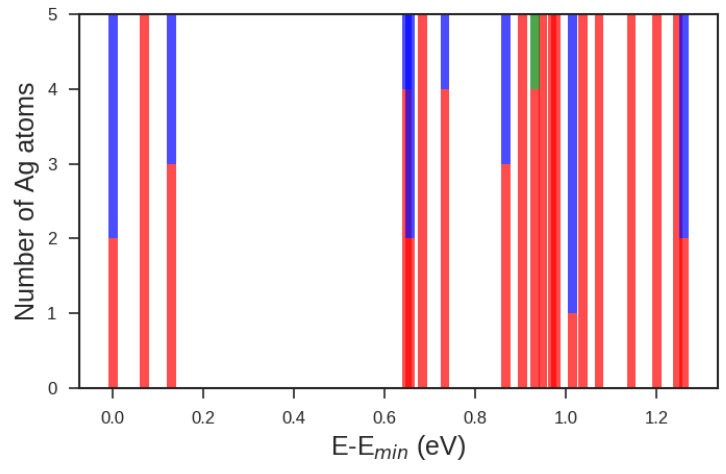

(c)

Figure A.2: Structural trends in (a) 3, (b) 4, (c)5-Ag alloyed $\mathrm{Au}_{25}\left(\mathrm{SCH}_{2} \mathrm{CH}_{2} \mathrm{Ph}\right)_{18}$ 


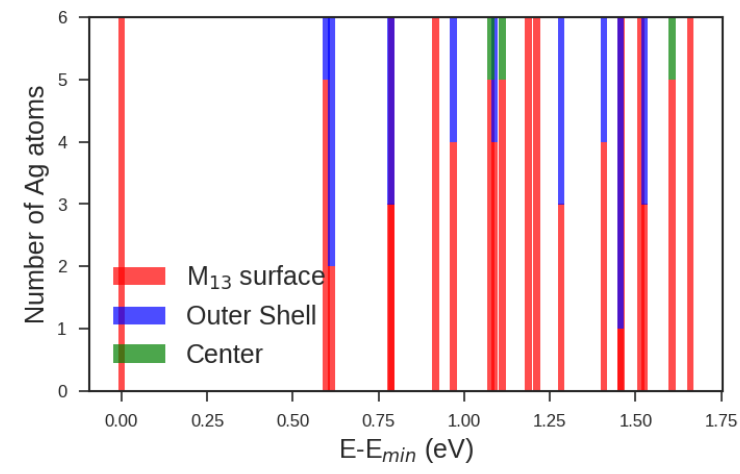

(a)

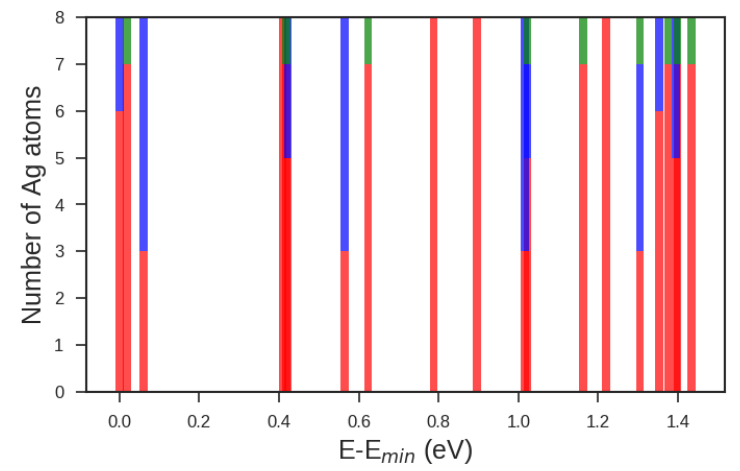

(c)

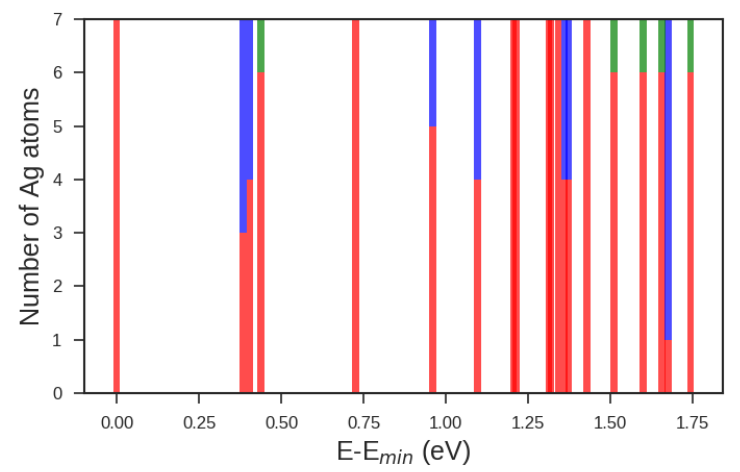

(b)

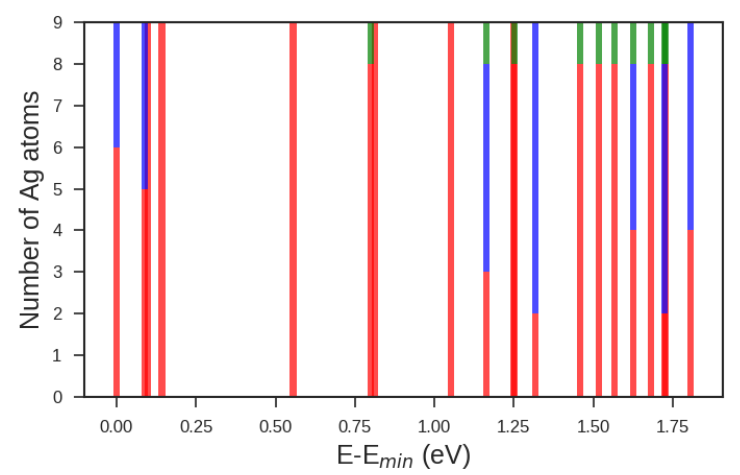

(d)

Figure A.3: Structural trends in (a) 6, (b) 7, (c)8, (d)9-Ag alloyed $\mathrm{Au}_{25}\left(\mathrm{SCH}_{2} \mathrm{CH}_{2} \mathrm{Ph}\right)_{18}$ 


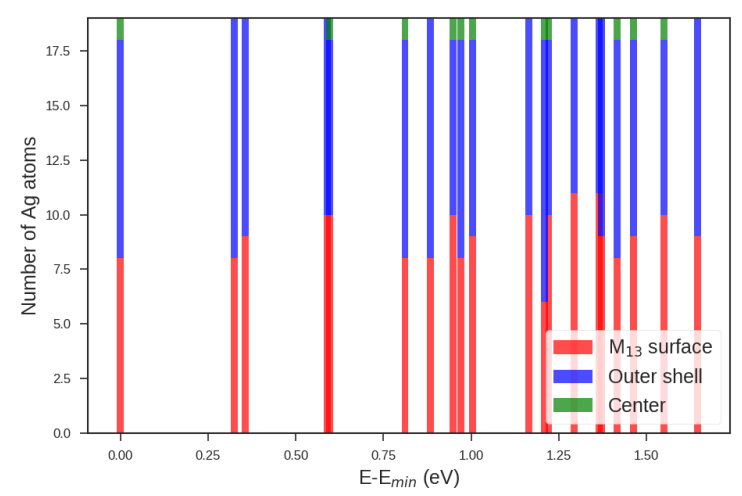

(a)

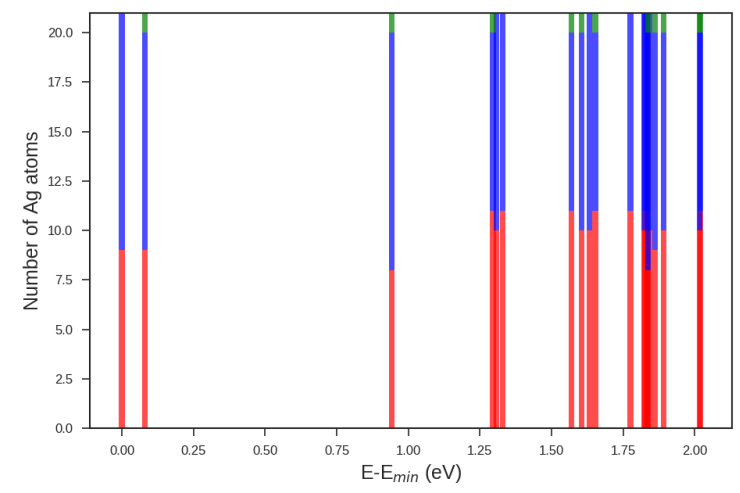

(c)

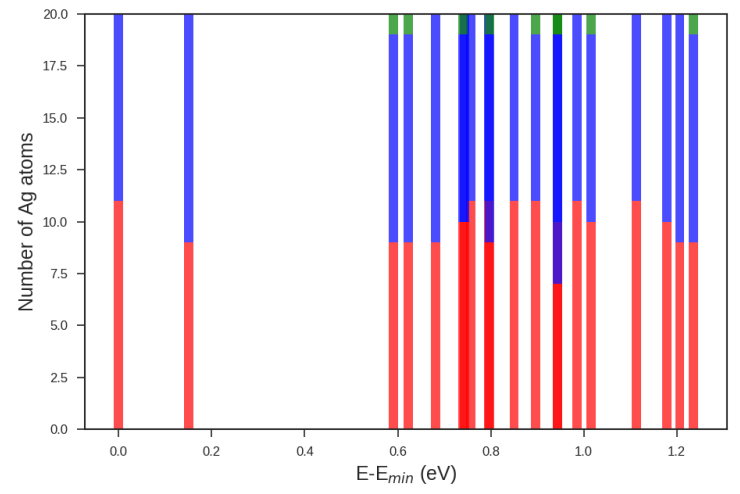

(b)

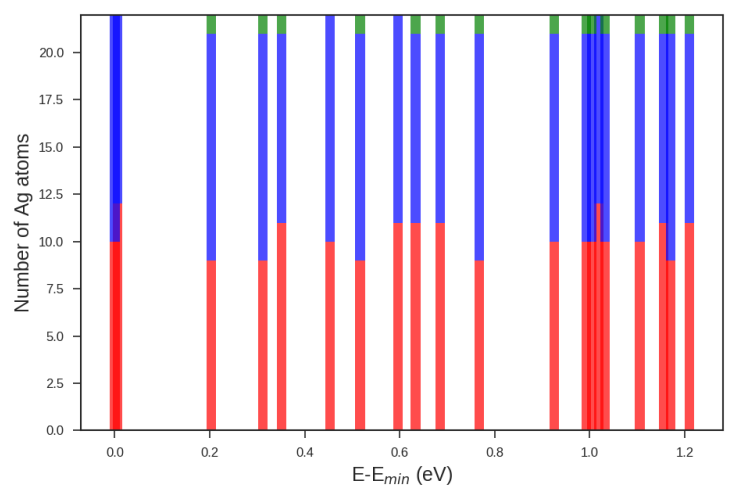

(d)

Figure A.4: Ag locations in (a) 19,(b) 20, (c) 21 and (d)22-Ag alloyed $\mathrm{Au}_{25}\left(\mathrm{SC}_{6} \mathrm{H}_{11}\right)_{18}$ 


\section{APPENDIX B}

Details of the atomic orbital basis set. We have adopted double numerical $\mathrm{sp}^{3}$ basis sets for $\mathrm{C}$ (cutoff radii of $r_{c}(\mathrm{~s})=4.3$ a.u. and $r_{c}(\mathrm{p})=4.7$ a.u.) , $\mathrm{O}$ (cutoff radii of $r_{c}(\mathrm{~s})=3.6$ a.u. and $r_{c}(\mathrm{p})=4.0$ a.u.) and $\mathrm{S}$ (cutoff radii of $r_{c}(\mathrm{~s})=4.5$ a.u. and $r_{c}(\mathrm{p})=5.0$ a.u.); whereas we use a minimal basis set is used for $\mathrm{H}$ (cutoff radius of $r_{c}(\mathrm{~s})=4.1$ a.u.). A polarized $\mathrm{sp}^{3} \mathrm{~d}^{5}$ basis set is used for $\mathrm{Ag}$ (cutoff radii of $r_{c}(\mathrm{~s})=5.6$ a.u., $r_{c}(\mathrm{p})=5.9$ a.u. and $r_{c}(\mathrm{~d})=4.9$ a.u.) and $\mathrm{Au}$ (cutoff radii of $r_{c}$ $(\mathrm{s})=5.4$ a.u., $r_{c}(\mathrm{p})=5.8$ a.u. and $r_{c}(\mathrm{~d})=5.0$ a.u. $)$

Random forests. Random forests can be used for classification and regression tasks. In both cases, the process of growing the forest and making predictions is similar. In simplest terms, a random forest is a collection of decision trees and these can be divided into classification and regression trees depending on the task at hand.

To understand how random forests work, we first have to understand how a decision tree works. As the prediction of the adsorption energies is a regression task, I will start by explaining how a regression tree works. For simplicity, consider a dataset with a single feature $x$. The dependence of the target variable on $\mathrm{x}$ is plotted in Figure B.1 (a). To grow our tree, we have to split the dataset into different regions based on the $x$ values. By simply looking at the data 4 split values can be identified. 
I will describe how these values can be determined mathematically later. Suppose that our first split is at S2. The way to decide which value should be used to do the first split will be explained later. After the first split, the tree can be grown using other split points in each region as shown in Figure B.1 (b).
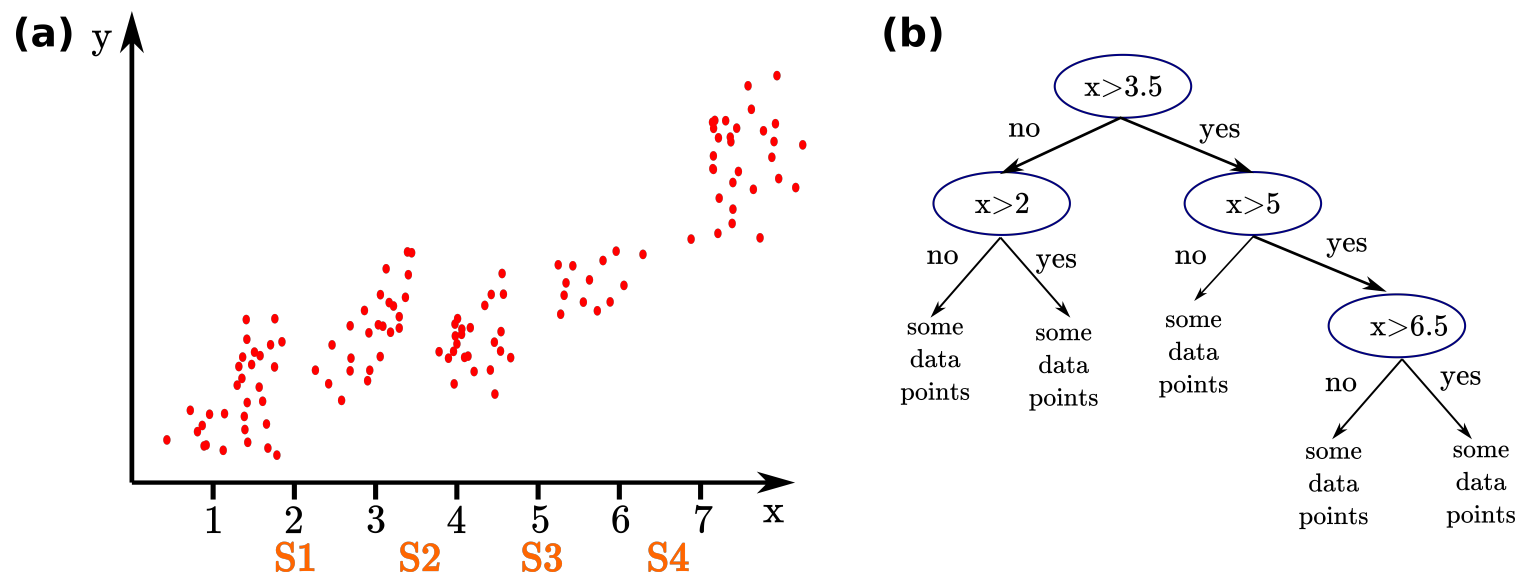

Figure B.1: (a) An arbitrary set of data points corresponding to feature $x$ and the (b) regression tree grown based on this data.

Now that we are done with splitting, we are left with data points at each leaf. The term leaf is used to identify the last node of a tree. Now remains the question, what is the value corresponding to each leaf node? There are several possibilities to arrive at these values. The simplest is to define the error estimate of the y values in a given region as the squared error. This definition leads to the best leaf node value of the average of the $y$ values in a region. Alternatively, one can fit a linear regression using the $y$ and $x$ values in a region, so that instead of fixed value as in the case of the squared error, we have an equation to obtain $y$ for a given $x$ belonging to a region. 
Once the tree is grown, we can predict the $y$ value for new $x$ value.

Now let's look at how to chose the first and the subsequent splitting values. To illustrate the important points of this process consider a feature space with three features, $x_{1}, x_{2}$ and $x_{3}$. We also have to define an error estimate for $y$. The most common and the one implemented in the scikit as the default is the mean squared error. For every feature $x_{j}$, select each of it's values as a possible splitting value and calculate the error of that split as,

$$
y_{\text {error }}=\frac{1}{N} \sum_{i: x_{j, k \leq S}}^{\max }\left(\bar{y}-y_{i}\right)^{2}+\frac{1}{N} \sum_{i: x_{j, k>S}}^{\max }\left(\bar{y}-y_{i}\right)^{2} .
$$

Here, $\bar{y}$ is the average of $\mathrm{y}$ values in a region. The feature with the minimum $y_{\text {error }}$ is selected as the first node. The same process is repeated to find the other nodes in the tree. 

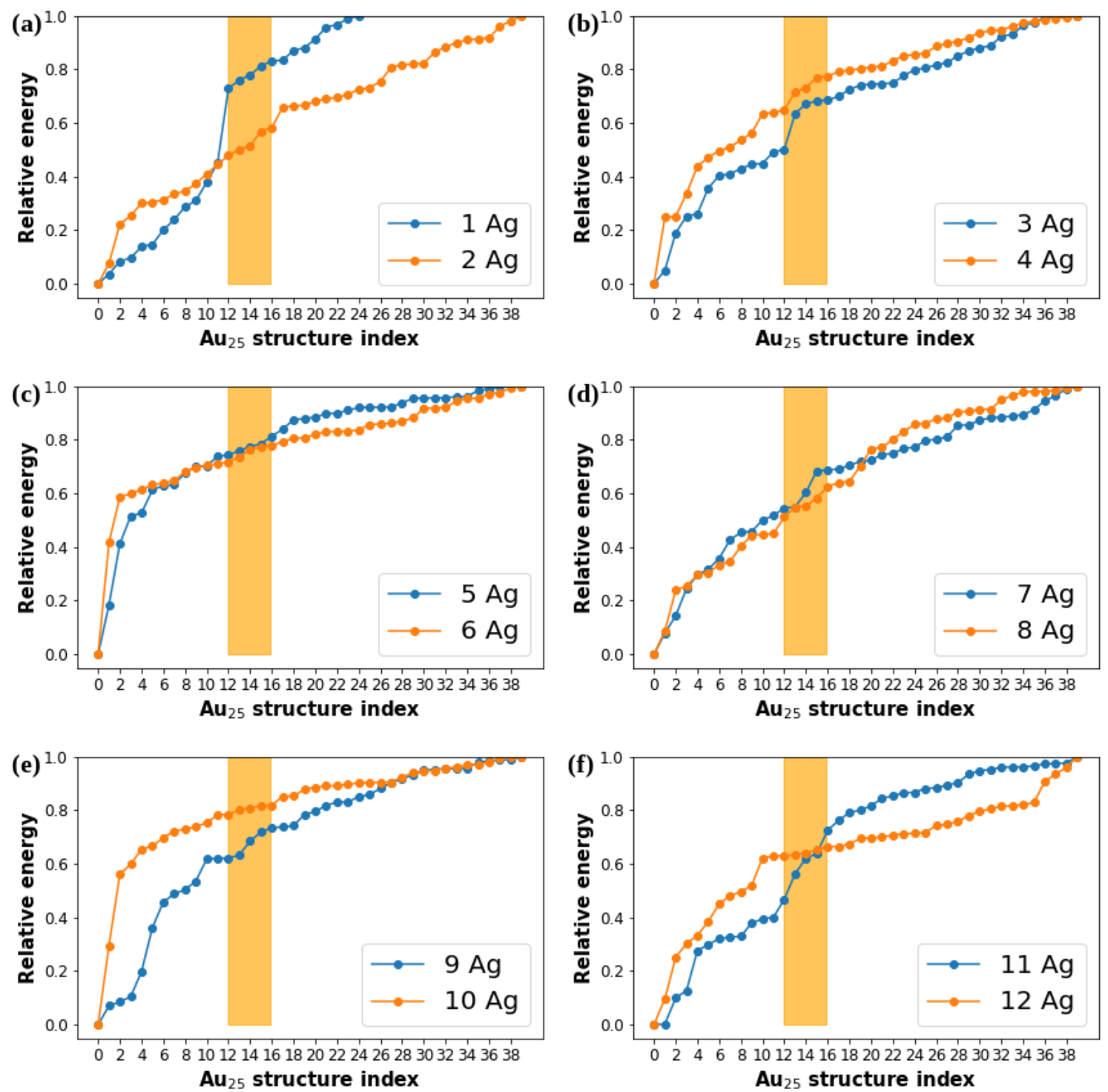

Figure B.2: Normalized relative energies of the lowest energy isomers for alloying levels 1-12. The structure index increases with the increasing relative energy. For example, the lowest energy structure has the index of 0 and the second lowest energy structure is given in the index 1 and so on. 

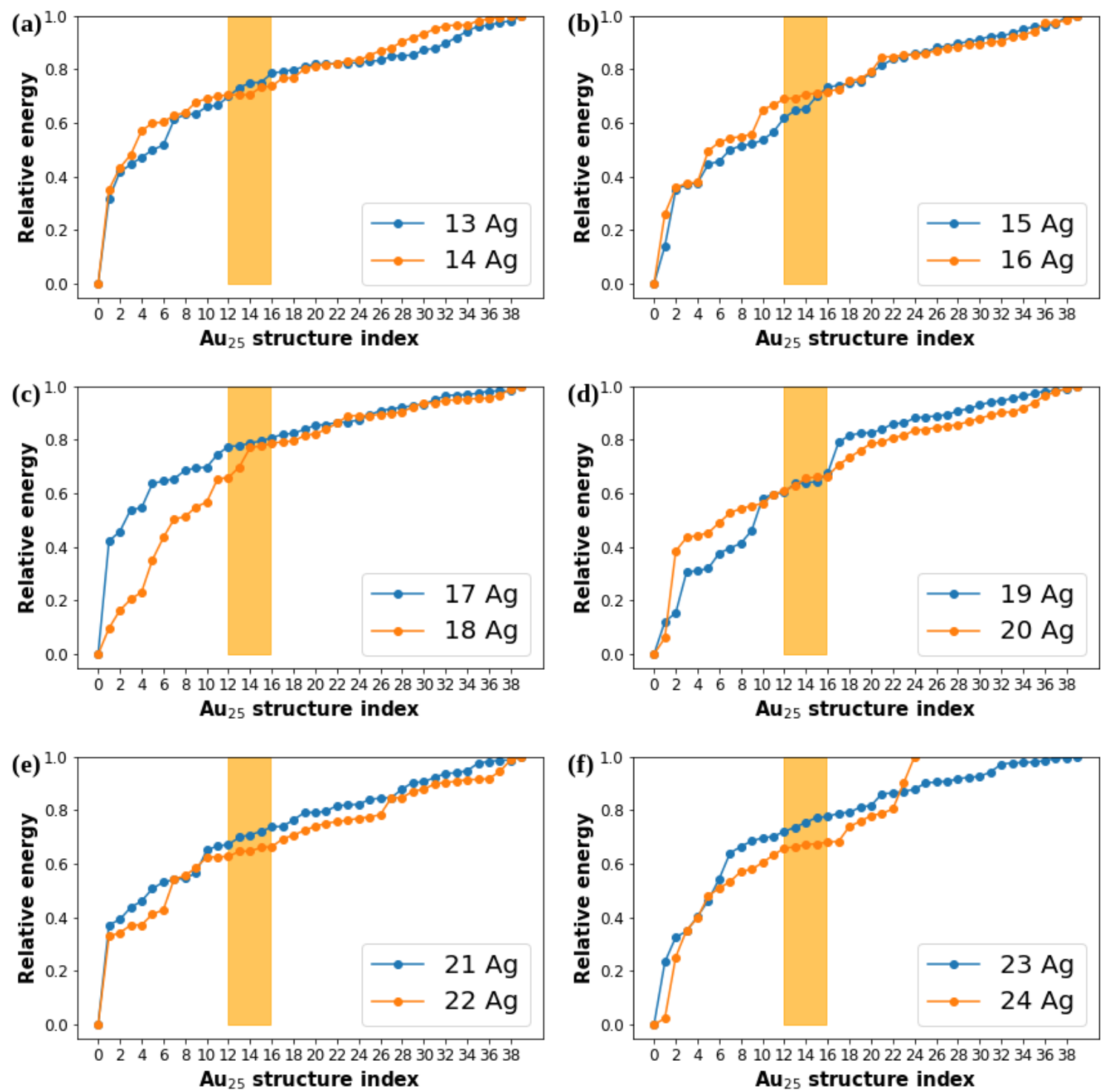

Figure B.3: Normalized relative energies of the lowest energy isomers for alloying levels 13-24. 


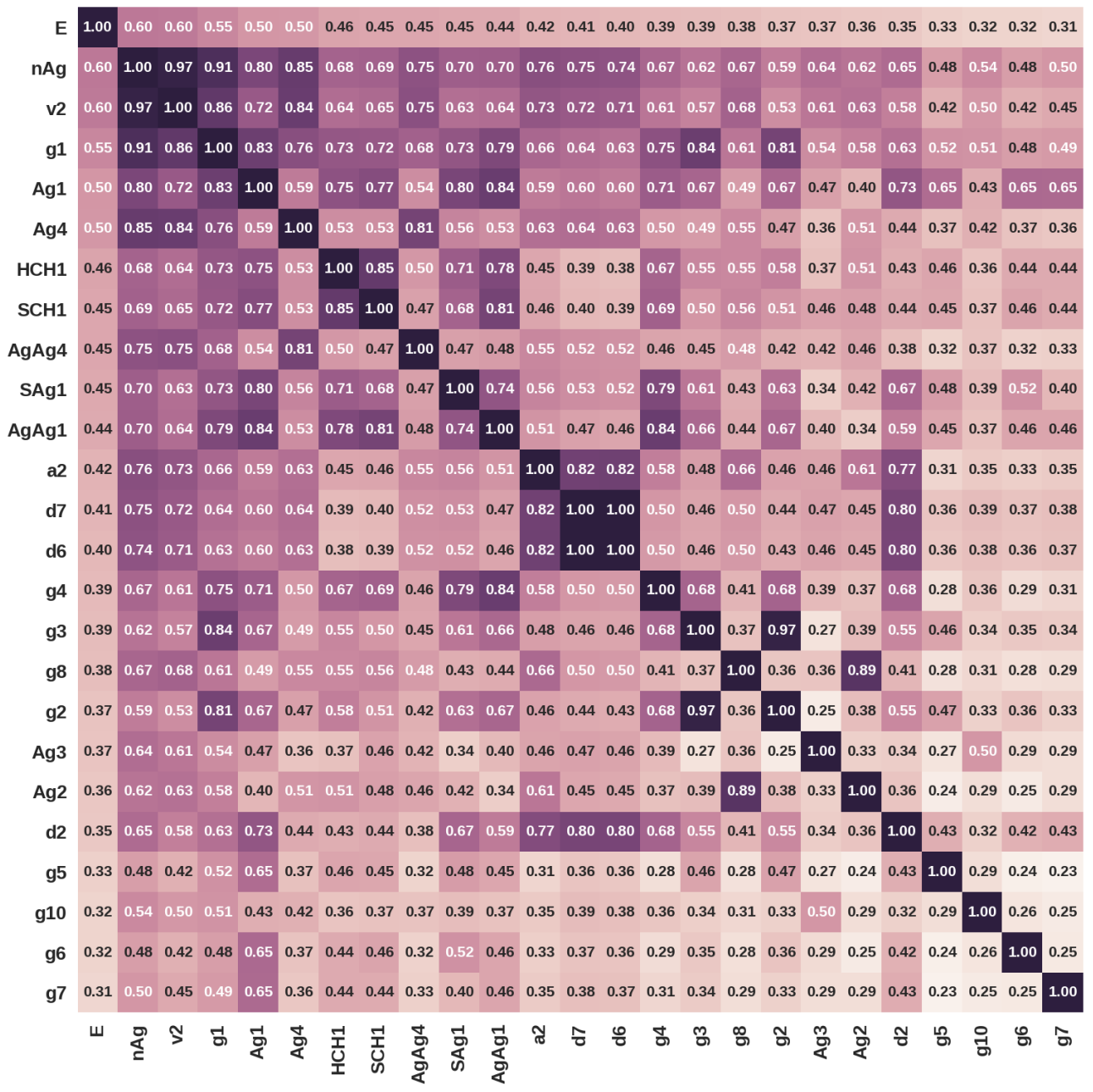

Figure B.4: Extended feature-feature Pearson correlation map.

Cross Validation.In k-fold cross validation, the training data set is split into nequal parts. Then one of the components is chosen as the testing set and the rest of the n-1components as the training set; machine learning method is applied to tune the 
model parameters. This process is carried out ntimes until all the ncomponents are used as the testing set.1Cross validation reduces the chances of over fitting and increases the generality of the parameters used in the machine learning model. Table S1 shows how the accuracy changes in the cross validation loop.

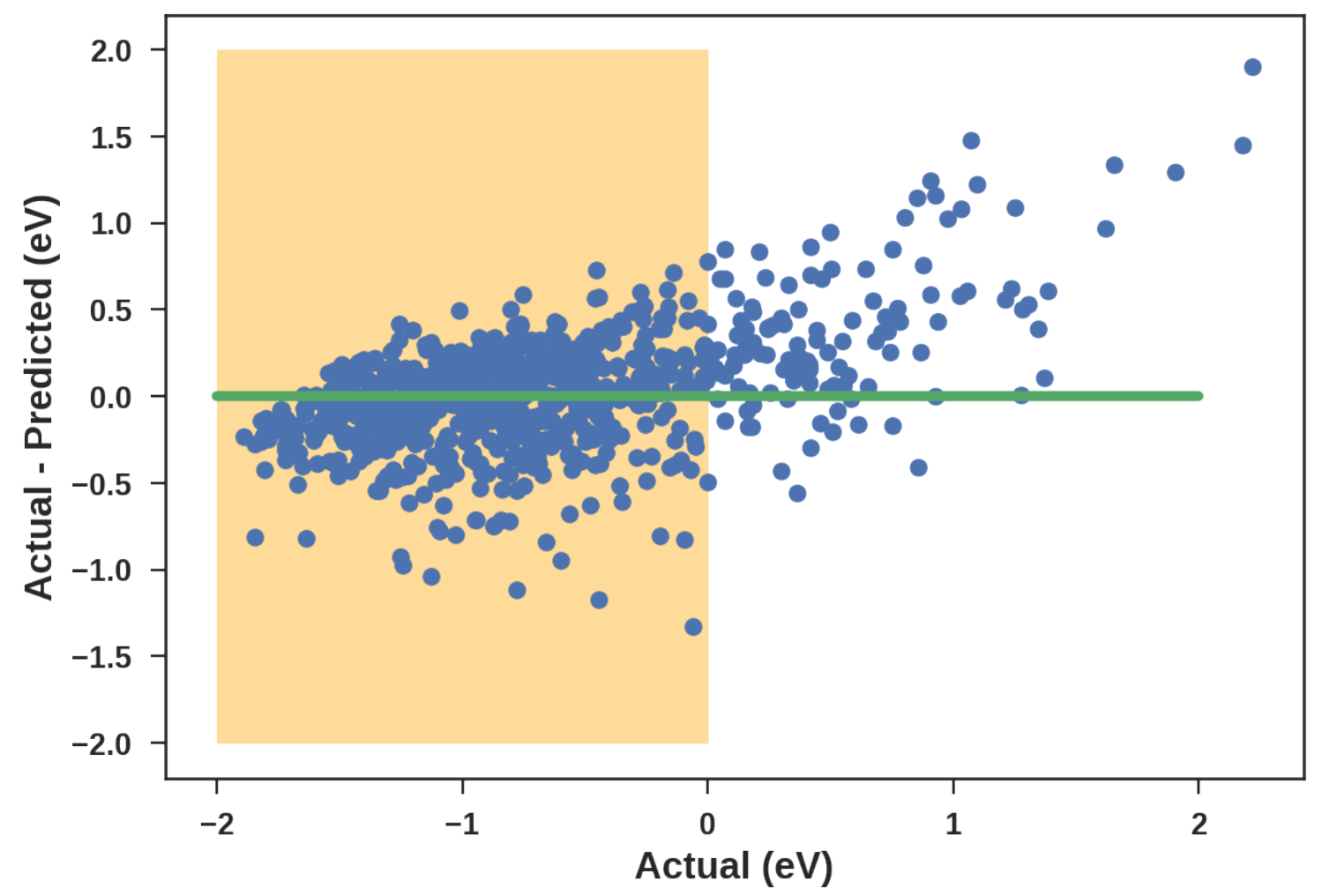

Figure B.5: Residual error vs the actual adsorption energy for $\mathrm{Au}_{25}$. The region corresponding to the negative adsorption energies is shaded in orange.

Prediction Performance for Au36 and Au133 Nanoclusters (eV analysis). Prediction accuracies for $\mathrm{Au}^{36}$ are $0.69659\left(\mathrm{R}^{2}\right), 0.26071 \mathrm{eV}$ (RMSE) and 0.18557 $\mathrm{eV}$ (MAE). And, for $\mathrm{Au}^{133}$, are $0.72171\left(\mathrm{R}^{2}\right), 0.42905 \mathrm{eV}$ (RMSE) and $0.27916 \mathrm{eV}$ 
(MAE).

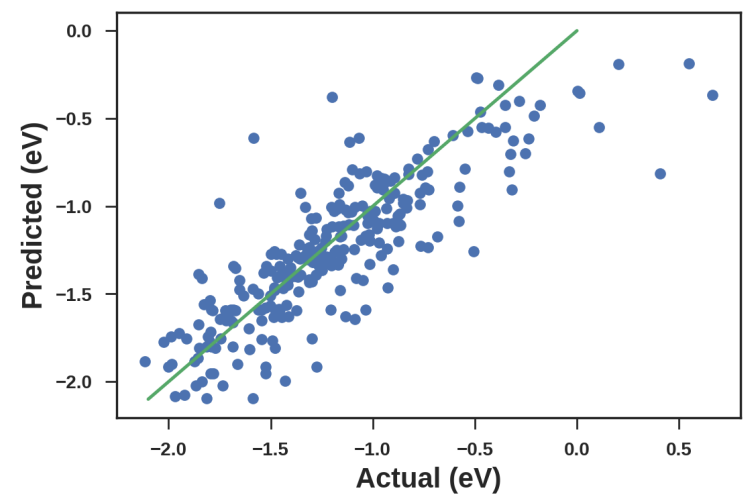

(a)

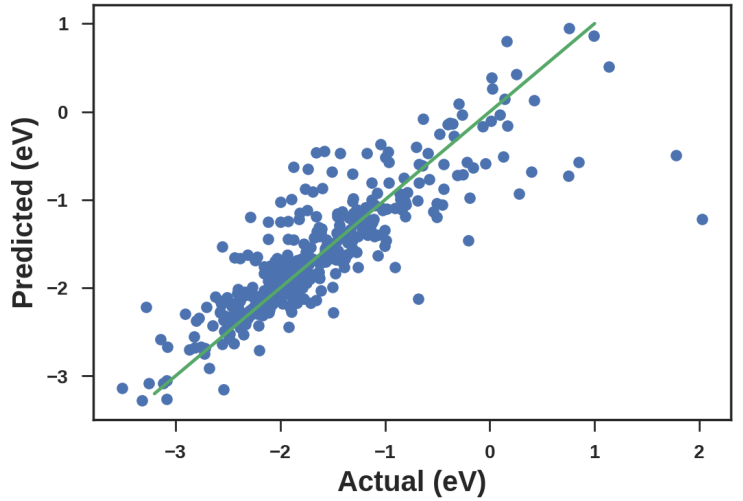

(b)

Figure B.6: Actual vs predicted adsorption energies of (a) $\mathrm{Au}_{36}$ and (b) $\mathrm{Au}_{133}$ nanoclusters in terms of electronvolts.
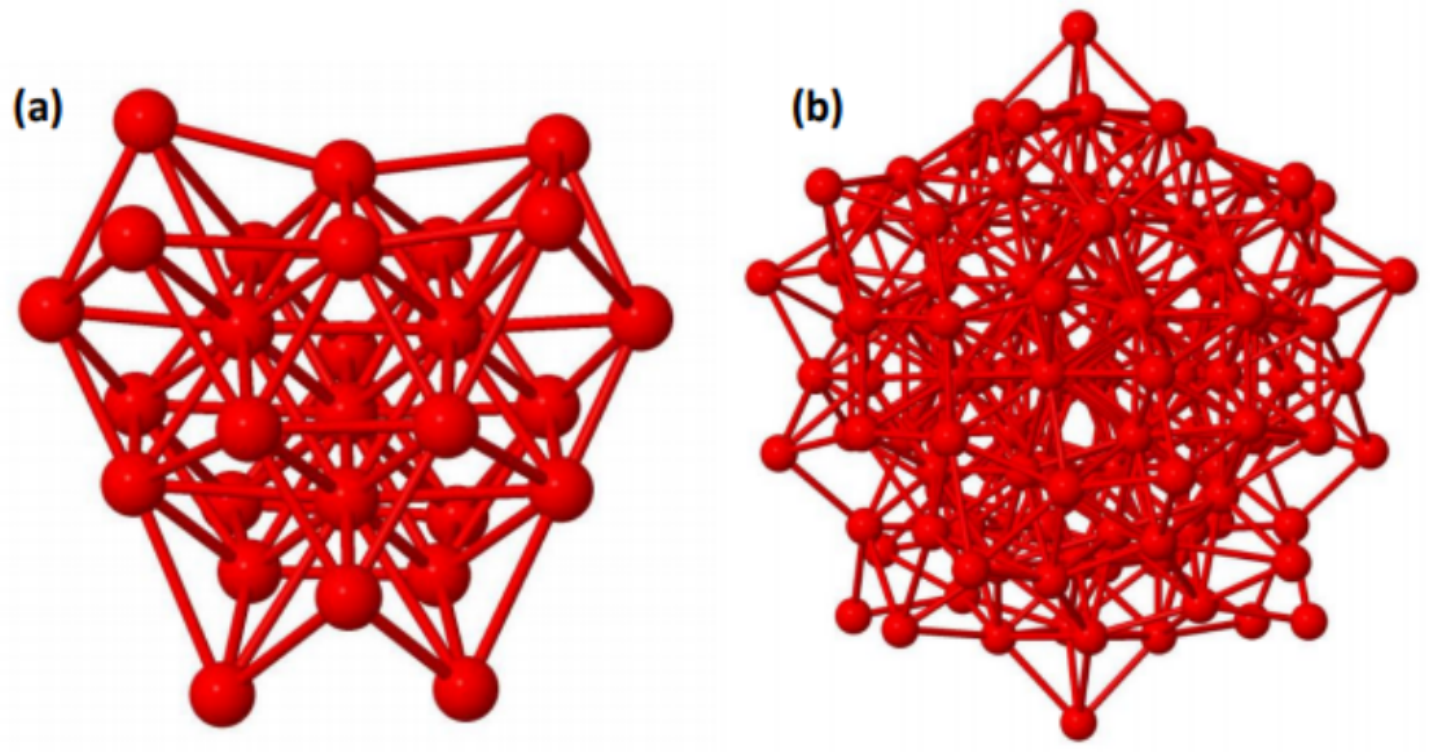

Figure B.7: $\mathrm{Au}$ cores of (a) $\mathrm{Au}_{36}$ and (b) $\mathrm{Au}_{133}$ nanoclusters. 
Sphericity calculation. Sphericity is defined as $\left(\pi^{1 / 3}(6 V)^{2 / 3}\right) / A$, where $\mathrm{V}$ and A are the volume and the surface area of the nanocluster. ${ }^{216}$ Volume was calculated using the ConvexHull function of the python library Scipy. ${ }^{242}$ To calculate the area, we found all the triangular facets forming the surface of the core and calculated the total area of these triangles.

The effect of sampling method on the model accuracy. The model accuracy depends on the current random sampling method used to generate Ag-alloyed structures because this sampling method does not guarantee the selection of all the lowest energy isomers for a given alloying level. It is not practical to generate all the possible isomers, a better sampling method - probably based on genetic algorithms - helps to generate more of the lowest energy isomers. A prediction done using these isomers is likely to result in better prediction accuracy.

\section{Features designed specifically based on the geometry of thiolated nan- oclusters.}

- Total electron affinity of each layer: Electron affinity is the tendency of a neutral atom to gain electrons. Therefore total electron affinity of all the atoms in a layer can provide information on chemical interactions between the adsorbate and the adsorption site. These values are readily available in data tables.

- Angle formed by each of the Ag dopants, center atom and the adsorption site: The purpose of this feature is to obtain information about the orientation of 
the ligands. As we do not use relaxed isomers to generate features, information given by this feature is limited and does not vary over different $\mathrm{Ag}$ alloyed isomers.

- Distance between adsorption site and each of the dopant atoms: This is another feature designed to find the effect of dopant atoms. The dimension of this feature vector is equal to the number of $\mathrm{Au}$ atoms. Distances calculated are sorted in the ascending order.

- Dihedral angle formed by $\mathrm{C}$ and $\mathrm{S}$ atoms connected to the adsorption site: This is another feature designed to capture the ligand effects on the adsorption energy.

- Angle formed by the $\mathrm{S}$ atoms connected to the adsorption site: This feature is also intended to model the ligand environment.

- Distance between the $\mathrm{H}$ atoms closest to the adsorption site. This feature is designed as a measure steric hindrance experienced by the adsorbates reaching the adsorption site.

- Statistical moments of the perpendicular distances to the Ag dopants from the line connecting the adsorption site and the center of mass of the cluster.

- Bin heights of a histogram: This kind of features can be created for any distribution of values. For example, consider the the distribution of distances between the adsorption site and the dopant atoms. After sorting these values 
in any desired order, we can create histogram of them containing a specific number of bins. The heights of the bins can be used as features. A pair correlation function constructed using atom-atom distances is another example for a distribution that can be used for this kind of binning. 


\section{APPENDIX C}

Position of Fe atoms in the supercell. Atoms connected with a solid line indicates that they are on the same (000) plane. Different colors of these colors represent the distance between two Fe atoms; blue: 1 nearest neighbor; green: 3 nearest neighbors; red: 3 nearest neighbors; orange: 4 nearest neighbors. The dimensions of the drawn supercells are given in angstroms. 

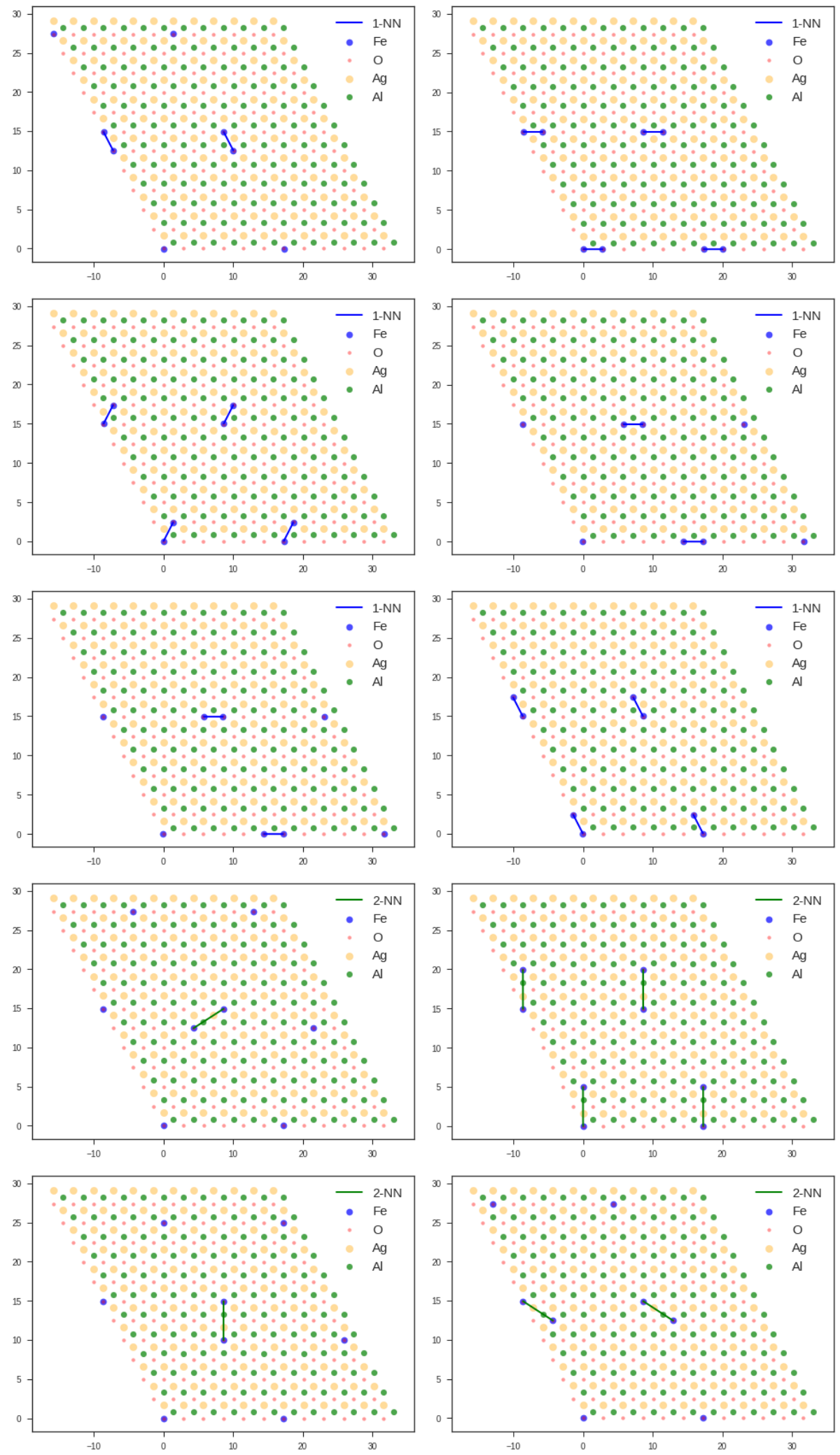

Figure C.1: Fe sites in 2\% Fe doped $\mathrm{AgAlFeO}_{2}$ 

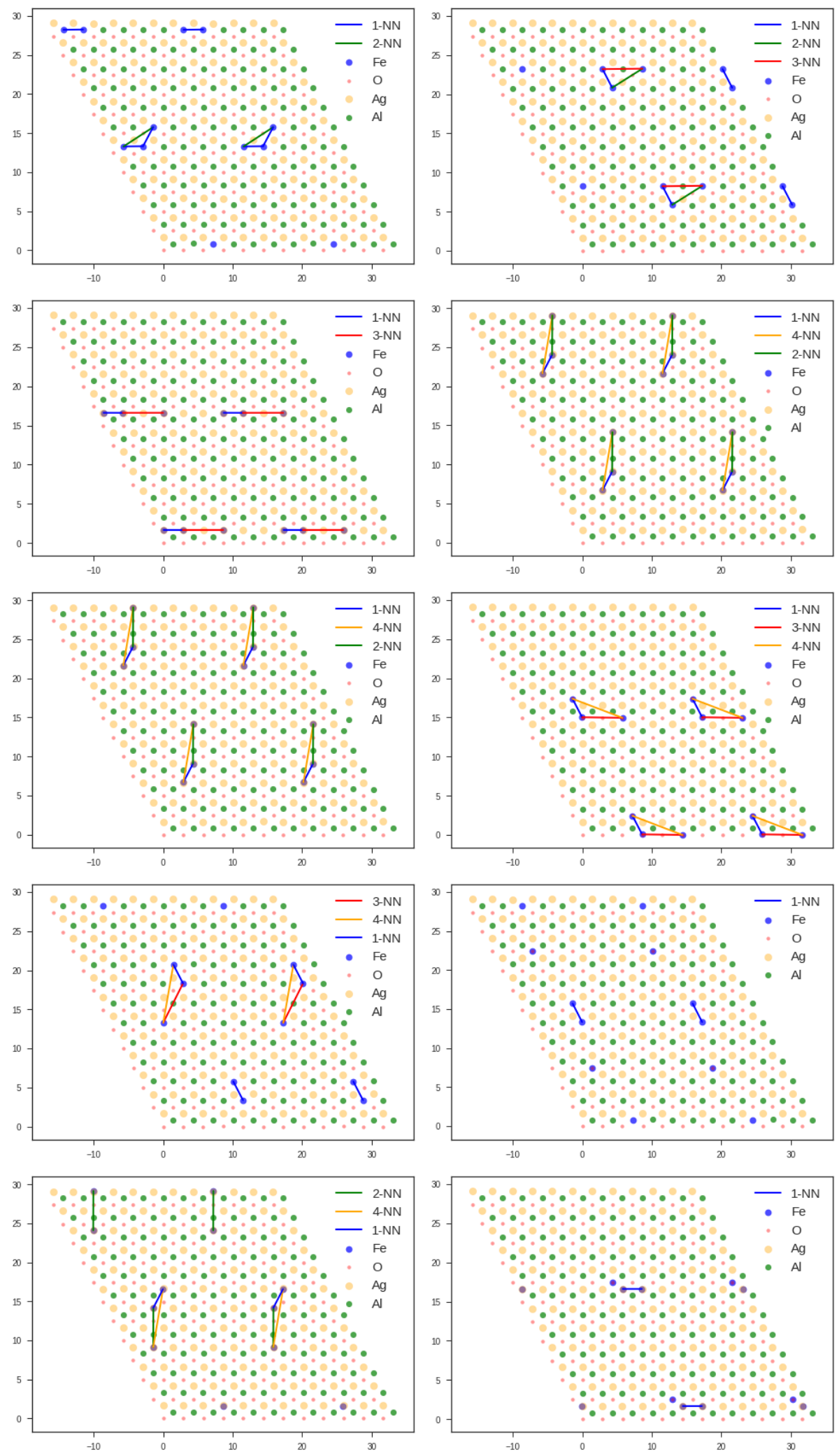

Figure C.2: Fe sites in 3\% Fe doped $\mathrm{AgAlFeO}_{2}$ 

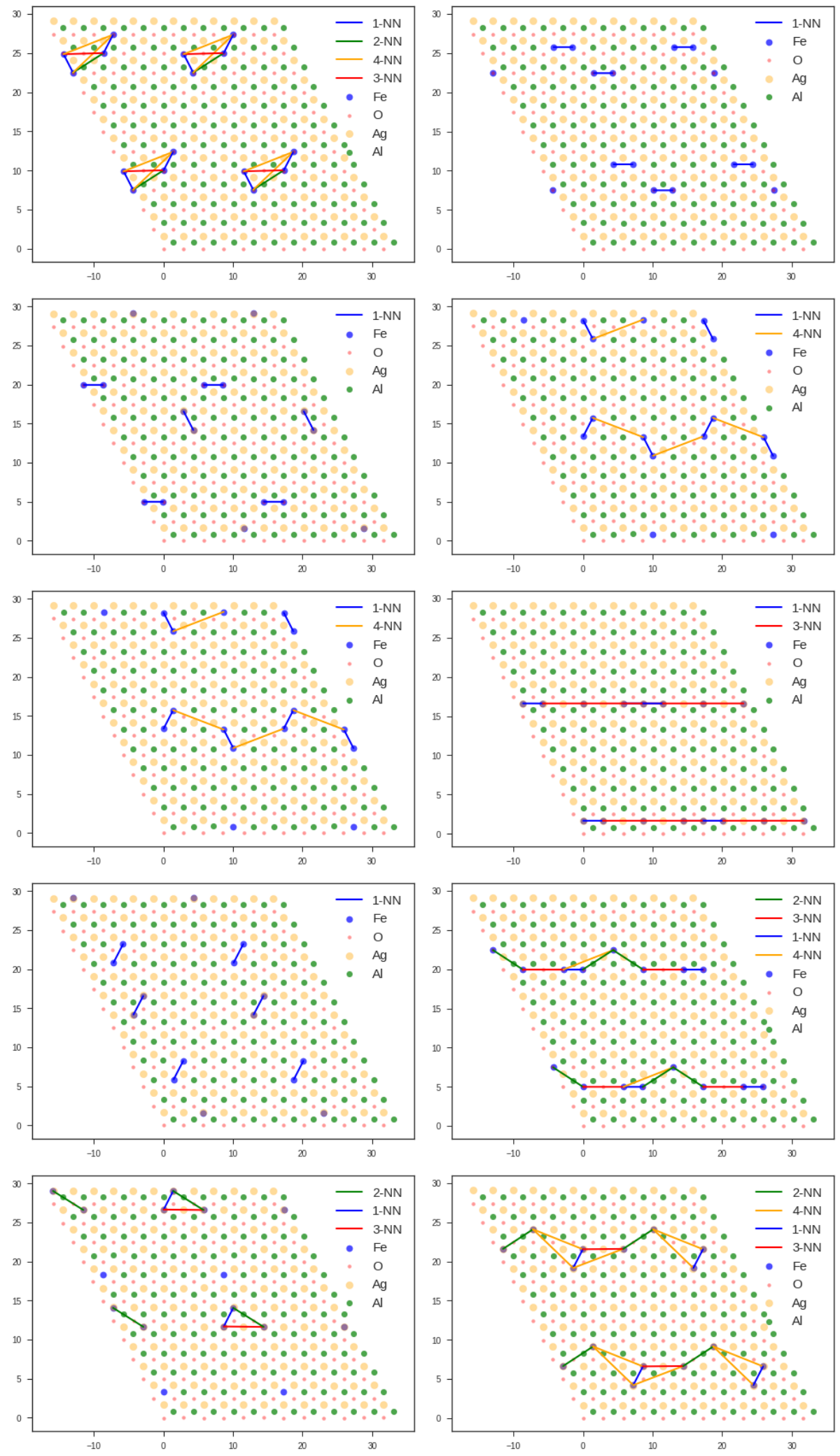

Figure C.3: Fe sites in 4\% Fe doped $\mathrm{AgAlFeO}_{2}$ 

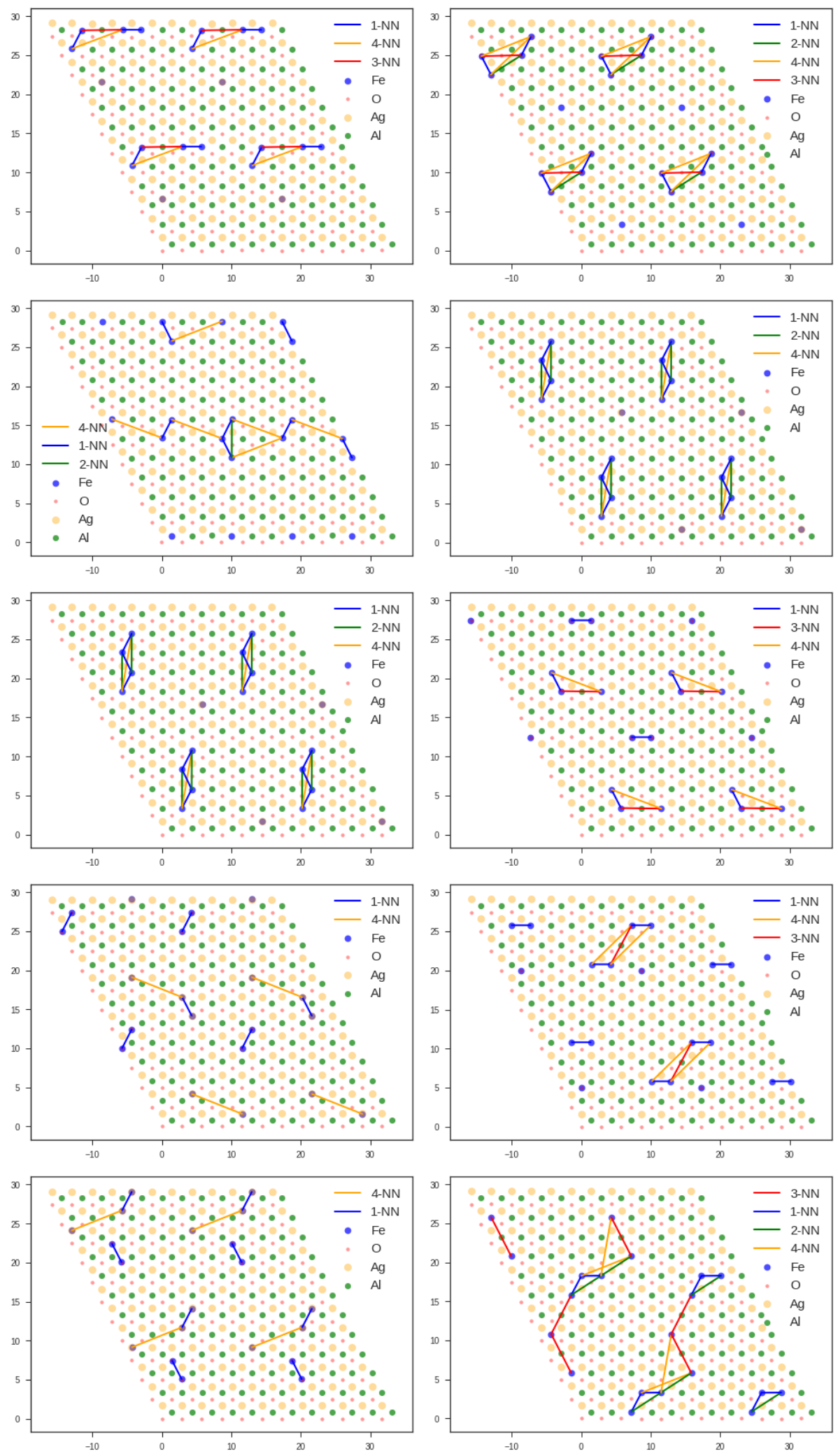

Figure C.4: Fe sites in 5\% Fe doped $\mathrm{AgAlFeO}_{2}$ 

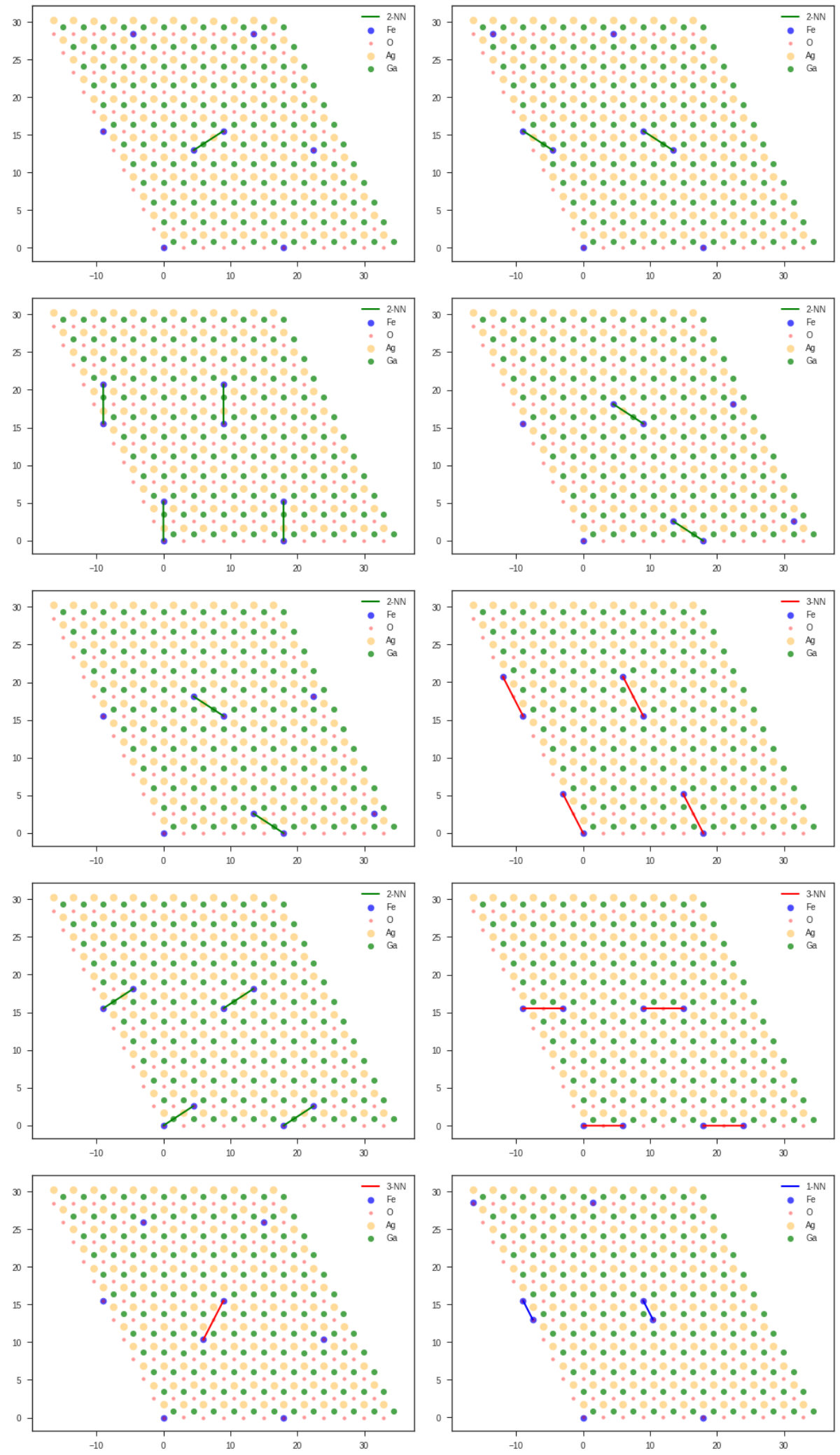

Figure C.5: Fe sites in 2\% Fe doped $\mathrm{AgGaFeO}_{2}$ 

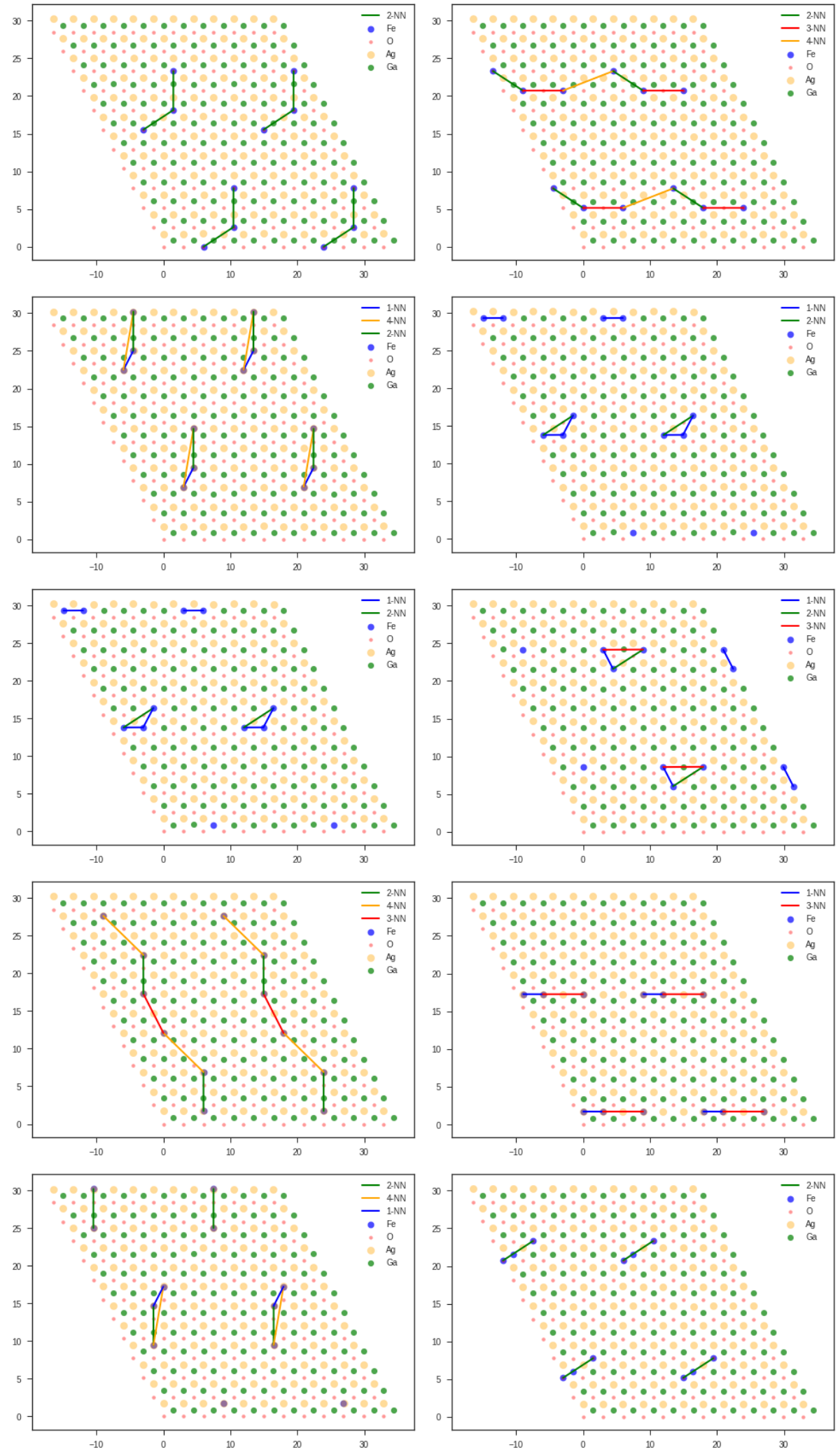

Figure C.6: Fe sites in 3\% Fe doped $\mathrm{AgGaFeO}_{2}$ 

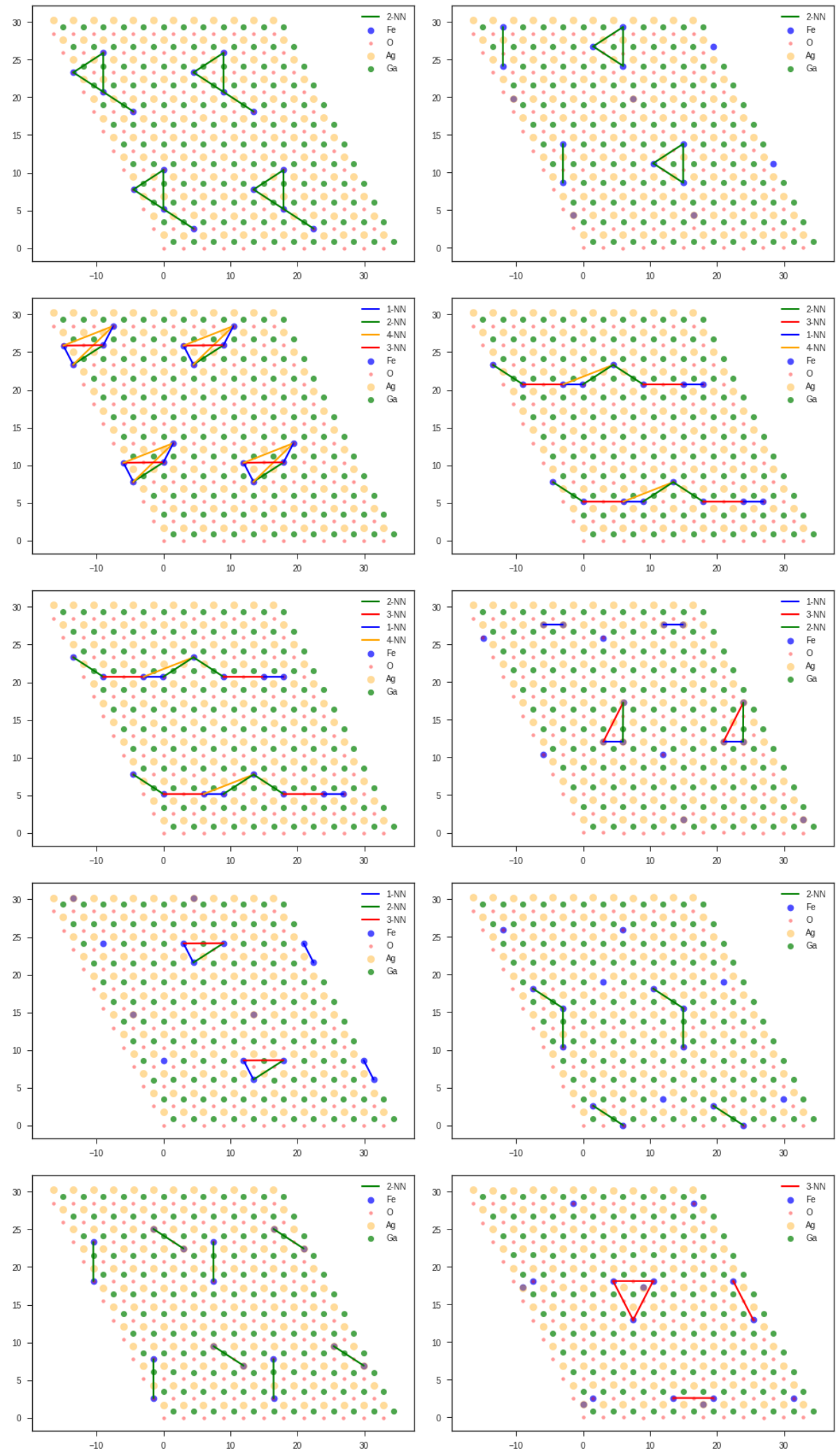

Figure C.7: Fe sites in 4\% Fe doped $\mathrm{AgGaFeO}_{2}$ 

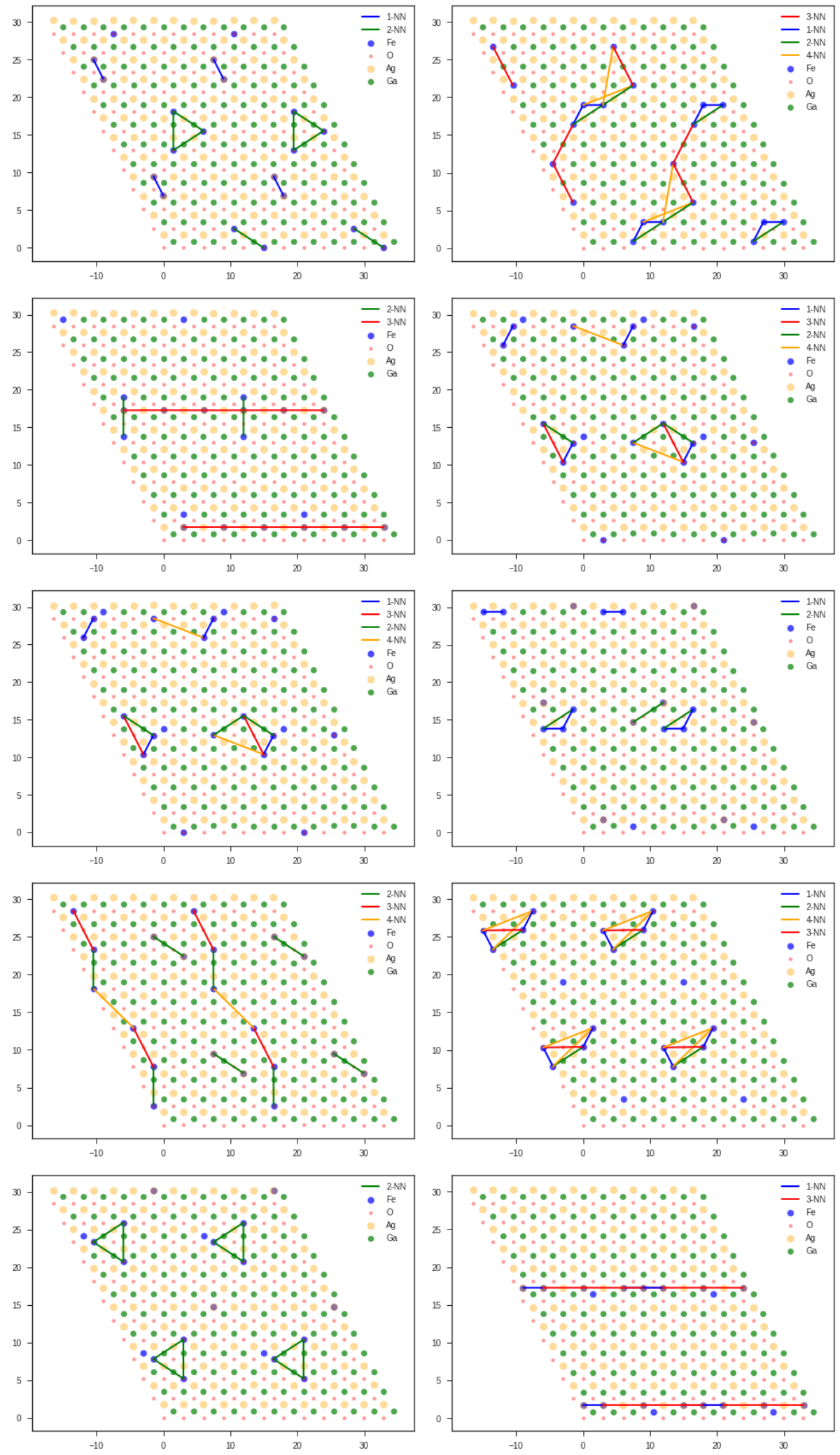

Figure C.8: Fe sites in 5\% Fe doped $\mathrm{AgGaFeO}_{2}$ 
Quantum Espresso calculations. To obtain band structures using Quantum Espresso, we considered a 96-atom supercell. This supercell was first structurally relaxed using FIREBALL until the root mean square force on atoms fell below 0.05 $\mathrm{eV} / \AA$, because relaxation calculations in Quantum Espresso requires a large amount computational resources as well as time. A Monkhorst-Pack grid the dimensions $4 \times$ $4 \times 1$ was used for the relaxation calculations. The FIREBALL-relaxed supercells were then subjected to one self-consisten-field calculations using Quantum Espresso $(4 \times 4 \times 1$ Monkhorst-Pack grid) to obtain the converged charge density required

for band structure calculations. In the last step a Quantum Espresso band structure calculation was performed for a reciprocal lattice path ( $k$-path) generated by the BandUP code.

Structural configuration of the lowest energy 96-atom supercells considered for unfolded band structure calculations. We used the bsym python package to find the symmetry inequivalent $8 \% \mathrm{Fe}$ doped structures of $\mathrm{AgAlO}_{2}$ and $\mathrm{AgGaO}_{2}$. For both the delafossites, the structure with the two Fe atoms positioned as nearest neighbors were found to have the lowest energy. 


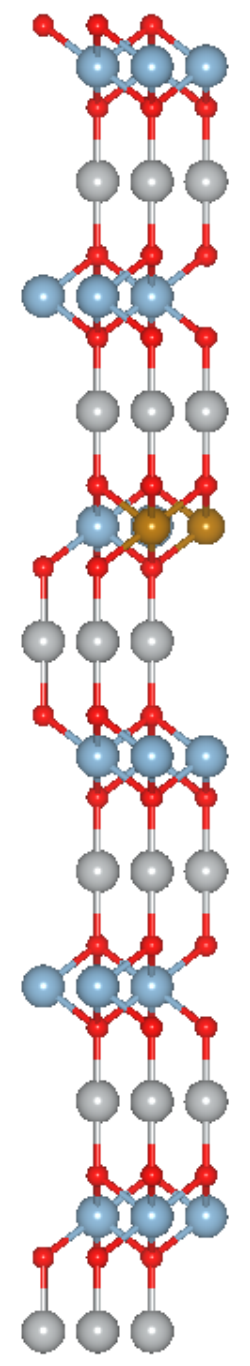

Figure C.9: The lowest energy $8 \%$ Fe doped configuration. Color code: Ag, grey; Al or Ga, light blue; Fe, orange; O, red. 